Nova Southeastern University

NSUWorks

\title{
Effectiveness of Asynchronous Reference Services for Distance Learning Students Within Florida's Community College System
}

Patricia C. Profeta

Nova Southeastern University

This document is a product of extensive research conducted at the Nova Southeastern University College of Computing and Engineering. For more information on research and degree programs at the NSU College of Computing and Engineering, please click here.

Follow this and additional works at: https://nsuworks.nova.edu/gscis_etd

Part of the Computer Sciences Commons

\section{Share Feedback About This Item}

\section{NSUWorks Citation}

Patricia C. Profeta. 2006. Effectiveness of Asynchronous Reference Services for Distance Learning Students Within Florida's Community College System. Doctoral dissertation. Nova Southeastern University. Retrieved from NSUWorks, Graduate School of Computer and Information Sciences. (780)

https://nsuworks.nova.edu/gscis_etd/780.

This Dissertation is brought to you by the College of Computing and Engineering at NSUWorks. It has been accepted for inclusion in CCE Theses and Dissertations by an authorized administrator of NSUWorks. For more information, please contact nsuworks@nova.edu. 
Effectiveness of Asynchronous Reference Services for Distance Learning Students within Florida's Community College System

$$
\text { by }
$$

\author{
Patricia C. Profeta
}

A dissertation submitted in partial fulfillment of the requirements for the degree of Doctor of Philosophy

Graduate School of Computer and Information Sciences Nova Southeastern University 
UMI Number: 3212018

\section{UMI}

UMI Microform 3212018

Copyright 2006 by ProQuest Information and Learning Company. All rights reserved. This microform edition is protected against unauthorized copying under Title 17, United States Code.

ProQuest Information and Learning Company 300 North Zeeb Road

P.O. Box 1346

Ann Arbor, MI 48106-1346 
We hereby certify that this dissertation, submitted by Patricia C. Profeta, conforms to the acceptable standards and is fully adequate in scope and quality to fulfill the dissertation requirements for the degree of Doctor of Philosophy.

Steven D. Zink, Ph.D.

Date

Chairperson of Dissertation Committee

Maxine S. Cohen, Ph.D.

Date

Dissertation Committee Member

Laurie P. Dringus, Ph.D.

Date

Dissertation Committee Member

Approved:

Edward Lieblein, Ph.D.

Date

Dean, Graduate School of Computer and Information Sciences

Graduate School of Computer and Information Sciences

Nova Southeastern University 
An Abstract of a Dissertation Submitted to Nova Southeastern University in Partial Fulfillment of the Requirements for the Degree of Doctor of Philosophy

\section{Effectiveness of Asynchronous Reference Services for Distance Learning Students within Florida's Community College System \\ by \\ Patricia C. Profeta}

January 2006

The impact of distance learning on higher education and the need to provide equitable library services to students in the digital environment emerged as critical areas during the 1990s. Library services available to distance learning students included digital reference and instructional services, remote access to online research tools, database and research tutorials, interlibrary loan, and document delivery. Digital reference services appeared to be one of the more significant services proffered by academic libraries although these services were developed often without forethought to goals and assessment. The purpose of this study was to examine the adequacy of asynchronous e-mail reference services offered through Florida's 28 community college libraries and the contribution of these digital reference service providers to the students' online learning community. The researcher analyzed data obtained through an unobtrusive study of asynchronous digital reference services and interviews conducted with digital reference service providers.

Studies existed for traditional and telephone reference service; however, the literature lacked studies addressing asynchronous digital reference service. Results from the unobtrusive portion of this study showed that the researcher received 240 of a possible 392 responses from the digital reference service providers. The researcher scored $24 \%$ as accurate with source information, $4 \%$ as accurate without source information, $20 \%$ as partly accurate with source information, and $7 \%$ as partly accurate without source information. The students scored $48 \%$ as accurate with source information, $12 \%$ as accurate without source information, $17 \%$ as partly accurate with source information, and $9 \%$ as partly accurate without source information. Responses took anywhere from 6 seconds to 20 days. The communication techniques exercised by the DRSPs were substandard. The study resulted in recommendations for the areas of digital environment, unobtrusive methodology, standards, accuracy, measurements, online relationships, training of digital reference service providers, student training, institutional responsibility, and marketing. 


\section{Acknowledgements}

I express thanks to my dissertation advisor, Dr. Steven Zink, and dissertation committee members, Dr. Laurie Dringus and Dr. Maxine Cohen, for their support throughout my entire doctoral experience at Nova Southeastern University. Each of these individuals imbued professionalism, integrity, expertise, caring, and a sense of humor into their teaching and advisory roles.

I wish to acknowledge and thank the District Board of Trustees, President Massey, colleagues, and students at Indian River Community College who supported my desire to realize my educational goals. Their encouragement was unfaltering and motivating.

I would like to recognize the digital reference service providers within Florida's community college library system. Both the providers who assisted with the interview portion of my study and the providers who assisted unknowingly during the unobtrusive portion of the study contributed invaluable information to an important area within library and information science. Students, college employees, and community members receive unflagging support and assistance through their efforts.

I owe a debt of gratitude to the Knowledge Maniacs, my Nova Southeastern University SCIS cohort. Rick Beideman, Wendy McLain, Karen Pate, Andy Potter, and Lynette Ralph continue to offer their friendship and encouragement.

I also extend my thanks to Carmen, Celia, Denise, Myra, Sue, and Susan, my fellow library administrators, who believe that library support services are essential for the academic success of students. These women provide steadfast support, moments of laughter, words of wisdom, and friendship.

Special thanks go to my parents, Henry and Geraldine Budzinski, because they have espoused the value of education throughout my entire life. I would also like to thank my sisters, family, and friends for their encouragement and support. This dissertation is dedicated to my sister, Karen, as she has always been an inspiration to me. My greatest thanks go to my children, Katie and Michael, who unselfishly gave up many family hours to enable me to accomplish my dream. Katie and Michael, may you always value the gift of learning since it will help you to achieve your personal and professional goals. 


\title{
Table of Contents
}

\author{
Abstract iii \\ List of Tables vii \\ List of Figures viii
}

\section{Chapters}

\section{Introduction 1}

Problem Statement and Goal 1

Definitions 9

Assumptions 10

Limitations 11

Florida Community Colleges and their Libraries 12

Relevance and Significance 15

Summary 18

\section{Review of the Literature 20}

Introduction 20

Accrediting Associations and Other Organizations 20

Impact of Digital Learning 23

Online Learning Community 23

Library Support Services for Distance Learning 27

Student Skills 29

Communication 30

Philosophy of Reference Service 33

Successful Digital Reference Services 34

Asynchronous Service Models 35

Standards for Digital Reference Service 36

Policies for Digital Reference Service 39

Advantages of Asynchronous Reference Services 40

Disadvantages of Asynchronous Reference Services 47

Staffing of Digital Reference Services 53

Reference Service 55

Reference Interview 56

Reference Questions 57

Reference Responses 61

Accuracy 62

Referrals 67 
User Satisfaction 67

Librarian Behavior 70

Turnaround Time 73

Assessment of Library Services 75

Measurements of Reference Services 81

Unobtrusive Testing 86

Barriers and Issues 93

Summary of the Known and the Unknown 96

Contributions to the Field 97

3. Methodology 98

Research Methods Employed 98

Specific Procedures Employed 100

Formats for Presenting Results 126

Resources Used 127

Reliability and Validity 128

Summary 130

\section{Results 131}

Analysis of the Data 131

Responses from the Digital Reference Service Providers 132

Background Details on the Unobtrusive Study 150

Results of the Unobtrusive Study of Asynchronous Reference Services 151

Response Rate 155

Analysis of the Answers 155

Summary of Results 222

5. Conclusion, Implications, Recommendations, and Summary 225

Conclusions 225

Implications 249

Recommendations 251

Summary 261

\section{Appendixes}

A. Methodology Workflow 272

B. Nova Southeastern University IRB Approval 276

C. Permission from IRCC 277

D. Permission from Library Administrators 278

E. IRB Adult/General Informed Consent [DRSP] 280

F. Checklist for Quality E-mail Reference Responses 282

G. Scoring Quality E-mail Reference Responses 285

H. Expert Panel 287

I. IRB Adult/General Informed Consent [student] 289

Reference List 292 


\section{List of Tables}

\section{Tables}

1. Response Rate for 14 Questions 152

2. Accurate Responses 153

3. Satisfaction Indicated by Willingness to Return to the DRSP 153

4. Additional Assistance and Referrals 154

5. DRSPs' Communication with Proxy 154

6. Significance of Researcher (X) vs. Student (Y) Scores - Communication 157

7. Communication Scores by Question/Size Researcher (R) vs. Student (S) 158

8. Significance of Researcher (X) vs. Student (Y) Scores - Accuracy/Satisfaction 159

9. Accuracy/Satisfaction Scores by Question/Size Researcher (R) vs. Student (S) 160

10. Timeliness Scores by Question/Size Actual Time vs. Business Hours (B) 162

11. Response Rate to Questions by Library Size 163

12. Comparison of Questions Received/Deemed Appropriate by DRSPs 229

13. Researcher/Student Comparison of Accurate and Satisfactory Responses to the 14 Questions 232

14. Student Assessors' Observations 237

15. Number of Institutions (by Size) Answering each Question 243 


\section{List of Figures}

\section{Figures}

1. Response Time and the Percentage of Questions Answered 152

2. Number of Questions Answered by Libraries 226 


\section{Chapter 1}

Introduction

\section{Problem Statement and Goal}

The U.S. Department of Education's National Center for Education Statistics (NCES) (2002) found that distance learning (DL) courses and programs offered through the Internet were more popular with postsecondary students than the other DL options of television or audio classes offered in live or prerecorded formats. Dunn (2001) estimated that within the next 10 years, nearly $95 \%$ of all educational providers recognized by the U.S. Department of Education would offer digitally enhanced instruction (p. 29). More than $55 \%$ of the nation's community colleges and universities already offered DL courses (Kiernan, 2003, p. 1). According to results released in a Sloan Foundation study, 1,170 academic officers indicated that enrollment during the fall 2003 semester reached 1.9 million students showing an increase of $19 \%$ from the fall 2002 with an expected enrollment of 2.6 million by the fall 2004 (Carlson, 2004, p. 1). The NCES (2004) specifically cited the enrollment growth at public two-year institutions where enrollment increased from 710,000 in 1997-1998 to almost 1.5 million in 2000-2001 (p. 85). Within Florida alone, DL enrollment at the community colleges increased by $47 \%$ for $2001-2002$ and at the universities by $46 \%$ for 2002-2003 (Armstrong, 2003, p. 5; Florida Department of Education, 2004b, p. 1). 
Of the 1,600 academic institutions surveyed by the NCES in 2000-2001, 90\% offered asynchronous delivery and 43\% offered synchronous delivery (Tabs, 2003, p. 11). DL's earlier appeal to administrators stemmed from the need for institutions of higher education (IHE) to take advantage of the opportunity before their students found it elsewhere (Christensen, 1997). The Web venue remained popular with higher education administrators as online courses eased overcrowding, reduced time spent on campus, enabled shared resources among faculty at various campus locations, offered more opportunities for less money, expanded course offerings beyond the physical limits of the campus, and provided students with another mode of learning (Coyner \& McCann, 2004; Florida Department of Education, 2002b; Lorenzetti, 2004; Singh \& Pan, 2004). Some students with learning disabilities, physical disabilities, low socio-economic status, or social disorders found the structure of DL courses facilitated their academic success (Collins, Schuster, \& Ludlow, 2002; Moisey, 2004; Sheets, 1992; Southern Regional Education Board, 2002a; Taylor, 2002). Galusha (1998) attributed the appeal of DL to the flexibility offered students struggling to balance the responsibilities of home, school, and work.

Literature identified student support services as necessary for students enrolled in Web-based programs and courses (Bauman, 2002; D’Angelo \& Maid, 1998; Lorenzetti, 2002a; Mills, Fisher, \& Stair, 2001; Restauri, 2004; Rumble, 2000; Southern Regional Education Board, 2002a; Tipton, 2001; White, 2001). Colleges and universities initiated the offering of DL programs; however, the capacity for these IHE to provide adequate support remained an issue (Dinwiddie \& Lillard, 2002; Lefor, Benke, \& Ting, 2003; Ludwig, 2002). Research suggested that adequate support offered a quality indicator and 
affected the success of an institution's DL program and its commitment to the DL student (Floyd \& Casey-Powell, 2004; McLean, 2000). Jackson and Parker (1998) further stated that quality support services ultimately influenced factors such as completion rates and funding opportunities. LaPadula (2003) maintained that support services provided an environment conducive to learning, improved retention statistics, enabled a student to compete in the electronic workplace, utilized those technologies prevalent in society, supported requirements of accrediting associations, and provided the DL student an equitable learning environment (p. 128).

To ensure adequate programs and courses in the Web environment, the nation's regional accrediting associations for colleges and universities stipulated that support services provided to DL students be comparable to those offered to campus-based students (Lebowitz, 1997). Student support services included library services and resources, admissions, financial aid, tutoring, registration, student rights, advising, technical support, health services, bookstore, and other services and resources. These same accrediting associations also mandated the use of systematic and ongoing assessment of support services to assure continued quality and adequacy. Accrediting associations strengthened the standards for DL library services because DL programs often failed or neglected to provide adequate library support services to students (Hufford, 2000, 2001; Lebowitz; Oregon University System, 1995; Pease \& Power, 1994; Southern Regional Education Board, 2002a). Gaide (2004a) considered library support services among the 10 best services offered by institutions for student completion (p. 8).

Recognition and support for quality DL support services appeared in an array of other documents and reports. The Principles of Good Practice for Electronically Offered 
Academic Degree and Certificate Programs, the standards for the Western Cooperative for Educational Telecommunications (WCET) (1996), set the tenor for quality DL. A subsequent policy report from WCET is entitled the Principles for Electronic Campus Library Services (Southern Regional Education Board, 2002b). The Institute for Higher Education Policy (2000) included the provision of library services in its report, Quality on the Line: Benchmarks for Success in Internet-Based Distance Education. The American Library Association (ALA) and the National University Extension Association (1931) recognized inadequate services to extension students in 1931 when it published a report dealing with the need to support the library needs of DL students. ALA's (2004a) Guidelines for Distance Learning Library Services asserted the need for "equivalent" library support and services to all students no matter where their learning takes place (p. 1). Guidelines and standards often helped an organization determine if its service was effective for accrediting standards (Lessin, McGinnis, \& Bean, 2002). With assessment measures so diverse, a national example might have helped to provide direction for local needs (Gross, McClure, Hodges, Graham, \& Lankes, 2001).

During the 1990s, more libraries started to offer DL resources and services comparable to those provided to campus-based students (Mahony, 1993). In 2000, the U.S. Department of Education identified $72 \%$ of public postsecondary institutions that offered digital reference via e-mail, including more than $65 \%$ of community colleges (Tabs, 2003, pp. 3, 51). Straw (2001) attributed the change to the advances in technology. Library services available to DL students included, but were not limited to, digital reference and instructional services, remote access to online research tools, database and research tutorials, interlibrary loan, and document delivery. Digital reference through 
both asynchronous and synchronous means became one response to accreditation requirements and ALA's recommendations. Researchers defined digital reference as a Web-based service providing mediated reference assistance to clients within an online environment (Lankes, 2004; Lankes, Gross, \& McClure, 2003; Pomerantz, 2003; Smith, 2003; Whitlatch, 2003).

According to Bargellini and Bordoni (2001), the advent of the Internet heralded the third generation of DL technology and asynchronous digital reference entered the DL environment during this phase. Digital reference services supplemented existing reference services. Many libraries began establishing asynchronous reference services when e-mail became available to them during the late 1980s and early 1990s with growth identified in the latter part of the 1990s (He \& Knee, 1995; Hill, Madarash-Hill, \& Bich, 2003; Middleton \& Peacock, 2000; Novotny, 2001; Schilling-Eccles \& Harzbecker, 1998). Johnson and McCarty (2001) believed that the need existed to offer digital reference primarily to support quality services to patrons. Colvin (2001) determined the change related to the decline of in-house reference questions, as well as a need to meet the patron where they required services. Researchers suggested libraries integrated asynchronous reference services into established reference practices (Corrigan, Diamond, \& Hill, 2002; Janes, 2004a).

Lankes, Gross, and McClure (2003) contended that libraries established digital reference services without forethought to goals and assessment. Bell and Levy (2004) believed that the profession was not overly "concerned with achieving customer satisfaction" (p. 158). Tennant (2003) and Wasik (2003) attributed the lack of assessment research to digital reference being in its infancy. Novotny (2001) stressed the need to 
conduct evaluations of new services to assess the level of service, as well as the extent of services provided. Despite the documented need to assess digital reference services routinely, the literature described mostly anecdotal observations and did not adequately address the effectiveness of digital reference service (Abels, 1996; Abels \& Liebscher, 1994; Barcellos, 2000; Bristow, 1992; Bristow \& Liu, 1999; Bushallow-Wilbur, DeVinney, \& Whitcomb, 1996; Clarke \& Brinkley, 2000; Garnsey \& Powell, 2000; Goetsch, Sowers, \& Todd, 1999; Gross, McClure, \& Lankes, 2001; Janes, Carter, \& Memmott, 1999; Lankes, 1998; Roysdon \& Elliott, 1988; Schilling-Eccles \& Harzbecker, 1998; Sloan, 1998b; Still \& Campbell, 1993; Whitaker, 1989).

Some researchers remained bothered by inconsistencies in service (Brownlee \& Ebbers, 2002; D’Angelo \& Maid, 1998). Hernon and Whitman (2000) pointed out that asynchronous reference services, the more established of the digital library services, needed further analysis to assess its effectiveness. Hastings and Tennant (1996) found that no rules existed and their development occurred as services progressed. Santovec (2002) identified the use of evaluations to measure success in DL programs. Barkley (1998) believed that the next step was to create and integrate guidelines into library practices.

McClure and Lankes (2001) emphasized the need for library administration to have tools necessary to determine success or weaknesses of services, including metrics and understandable data. Ryer and Nebeker (1999) commented that digital reference was a relatively unknown area for service quality. Stacy-Bates (2003) concurred by stating that a review of e-mail responses helped to measure the quality of asynchronous service. Katz (2001) stressed that technology allowed for efficient and accurate responses and 
librarians needed now to improve information retrieval skills. Horner and MichaudOystryk (1995) emphasized the need to conduct both quantitative and qualitative studies dealing with the efficiency of ready reference questions. McClure suggested that information regarding the "correct fill rate" for digital reference services was unknown and would have taken time to develop (Oder, 2001, p. 48). Gross, McClure, Hodges, Graham, \& Lankes (2001) believed that despite lack of formal measures, the written reference transaction remained useful for quality control assessments through administrative or peer evaluations.

Rodman (2001) believed such standards were on the verge of development. Kasowitz, Bennett, and Lankes (2000) found that little had been done to delineate standards for digital reference service and regular evaluation helped determine quality, accuracy, efficiency, and observance of established policies (p. 360). Standards were developing, but they needed adoption. Wasik (2003) agreed with the need for standards but found the lack of national standards created the need for institutions to create their own standards. With so many institutions offering digital reference service, criteria differed.

Ratcliff (1996) stipulated the use of assessment to improve services for accountability purposes. It provided evidence that an institution offered students a quality learning experience leading to academic success (Banta, Lund, Black, \& Oblander, 1996;

LaPadula, 2003). Janes (2002a) received 648 responses from 1,507 libraries surveyed and learned that only $9 \%$ evaluated digital reference services (pp. 551-552). Libraries lacked standard criteria for offering quality digital reference services thereby impeding the ability to determine effective practices. 
The researcher asked the following questions. Do asynchronous library support services adequately address reference requests made digitally by students? Do librarians respond to students in a timely manner? Do they ask additional or follow-up questions to comprehend fully the student's research request? How do librarians respond to student inquiries? Do they provide students with accurate information? Do they cite the sources used to answer reference questions?

Do these services enhance the student's online learning community? Does the librarian initiate an open and inviting dialogue? Does the librarian attempt to personalize the experience for the student? Does the librarian invite the student to use the service again should additional questions arise?

This study examined the adequacy of asynchronous e-mail reference services offered to DL students at Florida's 28 community college libraries and the contribution of library staff to the students' online learning community. The goal and research questions addressed the adequacy of library support for students enrolled in distance learning courses within IHE. DL created a substantive change in the mode of educational delivery and produced the online learner. Since DL courses did not always require campus-based participation, accrediting associations stipulated the need for IHE to offer DL students services comparable to those offered to campus-based students. They also required IHE assess the adequacy of DL services to students.

Libraries were considered a student support service, so the requirement to provide adequate and comparable DL support service also affected them. Adequate and effective library services have caused problems for libraries historically. The profession already has documented challenges providing effective telephone and in-person reference 
services with both the accuracy of the responses and the treatment of customers. Digital reference services exacerbated the challenge because existing literature did not adequately address the effectiveness of digital reference for DL students, nor did it address how asynchronous library support services affected the academic experience of DL students.

\section{Definitions}

This study used the following definitions:

Reference is the "provision of information upon request, independent of format or medium" (Ladner \& Tillman, 1993, p. 45).

A reference transaction involves "the knowledge, use, recommendations, interpretation, or instruction in the use of one or more resources by a member of the library staff' (Association of Research Libraries, 2002, p. 5).

The reference interview is defined as the interchange between the student and the librarian whereby the librarian attempts to help the student find the requested information (Ammentorp \& Hummelshoj, 2001).

Digital reference service is reference service and assistance provided to an institution's clientele by library personnel through a Web-based environment using asynchronous means (e-mail) or synchronous means (virtual chat) (American Library Association, 2004a; Lankes, 2003; Pomerantz, 2003; Smith 2003; Whitlatch, 2003).

A digital reference service provider (DRSP) is a library employee with reference skills and expertise responsible for responding to the inquiry. 
An accurate response is one that provides the patron with a correct and current response to an inquiry and one that includes the source information used to generate the response (Kahn, 2001).

A successful response is one where the patron indicates satisfaction with the response provided by the DRSP, despite any erroneous information included in the response.

An unobtrusive study is one method of testing subjects without their knowledge or consent (Kaske \& Arnold, 2002).

Proxy is the term used to identify the researcher posing as a valid student.

\section{Assumptions}

The researcher assumed the following statements:

1. All of Florida's community college libraries offer digital reference service through asynchronous means (e-mail) to their clientele.

2. In many instances, professional librarians staff the asynchronous digital reference service desk.

3. Libraries serve all clientele, regardless of their affiliation with the institution.

4. DRSPs provide accurate and timely responses to all asynchronous inquiries.

5. DRSPs possess the ability to answer reference questions (Crowley \& Childers, 1971).

6. The asynchronous delivery method does not affect accuracy and currency of the responses.

7. DRSPs treat DL students with a friendly and responsive attitude. 
8. The use of unobtrusive testing simulates real life scenarios and reduces the “contamination” of results (Jirjees, 1981, p. 23).

The researcher further assumed the following statements with regard to this particular study:

1. Library administrators within Florida's community colleges will support and will recommend a DRSP library representative to be interviewed.

2. The recommended DRSP will assist with the study.

3. The DRSPs from each of the libraries will respond to each of the 15 questions.

4. IRCC students will assist with the study and help assess the responses.

\section{Limitations}

The study was limited to 28 community college libraries within the state of Florida enabling the researcher to utilize a readily available population; however, the adequacy of asynchronous e-mail reference services offered to DL impacted students in IHE nationwide. The results could not be generalized to all types of libraries, including other academic libraries, to all geographic locations in the U.S. and outside of the U.S., to all types of reference service, and to the performances rendered by individual librarians.

The proficiencies of the DRSPs were not predetermined (Dilevko \& Dolan, 1999). The types of questions asked represented questions consistently asked at the researcher's library; however, the questions may not be asked at other community college libraries in Florida (Gers \& Seward, 1985). The DRSPs had access to a similar set of resources with which to answer the inquiries; however, not all libraries had access to the same resources 
(Hernon \& McClure, 1987a; McClure, 1980). The unobtrusive nature of the study identified responses at particular moments in time and may not have fully represented the expertise of a DRSP at other times (Hernon \& McClure). DRSPs may have worked for one or more services thereby increasing the likelihood of discovery (Kaske \& Arnold, 2002). The assessments of the responses represented the judgments rendered by the researcher and students at the researcher's institution. Childers' suggested some subjectivity in determining the correctness of responses (Crowley \& Childers, 1971).

\section{Florida Community Colleges and their Libraries}

The 28 community colleges in Florida comprise the Florida Community College System (FLCCS). Local boards of trustees oversee the community colleges within Florida; however, the colleges fall under the authority of the State Board of Education (Florida Department of Education, 2002a). In 2003-2004, the student headcount for the FLCCS was 816,290 (Florida Department of Education, 2005, n.p.). Although DL enrollment at Florida's community colleges increased by $47 \%$ in $2001-2002$, and 26 of the 28 community colleges offered DL courses during spring 2005, students still commuted to community colleges within $90 \%$ of the state (Armstrong, 2003, p. 5; Florida Department of Education, 2005, n.p.). Each of the community colleges had a library with a Web presence, each library maintained an affiliation with Florida's College Center for Library Automation (CCLA), and each supported the Distance Learning Library Initiative's (DLLI) Reference and Referral Center.

The Report for the Florida Community College System: The Fact Book described the arrangement of the community colleges in Florida by size as determined by the number 
of full-time enrollments (Florida Department of Education, 2005). A number of community colleges awarded baccalaureate degrees in critically needed areas (i.e., nursing, technology management, and public safety), so they dropped the word community from their names; however, their missions still emphasized the associate degree programs. This study addressed the community college libraries by size of the institution since the small-sized group contained 10 colleges, the medium-sized group contained 9 colleges, and the large-sized group contained 9 colleges. During the study, the small-sized community colleges in Florida were Chipola, Florida Keys, Gulf Coast, Lake City, Lake-Sumter, North Florida, Pasco-Hernando, Polk, South Florida, and St. John's River. The medium-sized community colleges in Florida were Brevard, Edison, Central Florida, Manatee, Okaloosa-Walton, Pensacola, Santa Fe, Seminole, and Tallahassee. The large-sized community colleges in Florida were Broward, Daytona Beach, Florida at Jacksonville, Hillsborough, Indian River, Miami Dade, Palm Beach, St. Petersburg, and Valencia (Florida Department of Education, 2004a).

The College Center for Library Automation, contracted by the State, managed the shared library management system used by Florida's 28 community college libraries. Library personnel networked through regional training sessions and workshops, as well as statewide participation on committees established by the Executive Committee of the College Center for Library Automation. Additionally, the College Center for Library Automation provided oversight for the Library Information Network for Community Colleges (LINCC), the online catalog shared by the 28 community college libraries, and the library information portal (LINCCWeb) (College Center for Library Automation, 2003a, p. 1). The College Center for Library Automation's portal included a connection 
to the Web pages for each of the 28 community college libraries, as well as the "Ask a Librarian" digital reference service available through statewide synchronous chat and college-specific asynchronous e-mail.

The synchronous service resulted from a federal Library Services and Technology Act (LSTA) grant that the College Center for Library Automation and the Tampa Bay Library Consortium (TBLC) submitted in 2002, and it involved professionals from public, academic, and special libraries in Florida (College Center for Library Automation, 2002; College Center for Library Automation, 2003c). The service also included a full day of training and periodic interactive sessions with the AskA coordinators. Asynchronous service stemmed from the effort of each individual community college library; however, the College Center for Library Automation created a Web site providing links to both types of service. Although many of Florida's community colleges began offering asynchronous reference service at some time during the last 10 years, the ability to access both the synchronous chat reference service and the asynchronous e-mail reference service from one location debuted in July 2003 (College Center for Library Automation, $2001 ; 2003 b)$.

DLLI proved to be a collaborative venture among Florida's public universities, community colleges, and public libraries. Introduced in 1997, the venture helped to shape a statewide electronic resource sharing and document delivery program (Ault \& Viggiano, 2000; College Center for Library Automation, 1997; Madaus \& Webster, 1998). Statewide reciprocal borrowing between the community college and public universities also resulted from the Initiative. A statewide Reference and Referral Center, supplementing local services, opened in February 1998 during the early days of the 
Initiative and closed due to funding concerns (Madaus \& Webster; Smith, Race, \& Ault, 2001). The Center offered both synchronous and asynchronous reference service. The "Ask a Librarian's" live chat reference service supplanted the Reference and Referral Center.

\section{Relevance and Significance}

Research found support services, such as advising, library services, counseling, tutoring, social support, technical support, financial aid, recruitment, orientation, registration, and bookstore services, invaluable to the experience of the DL student for reasons such as reduced attrition, improved academic success, increased enrollment, and assimilation into the college environment (Dirr, 1999, p. 1; Roberts \& Davey, 2002). Sherritt (1996), Johnson (1999), and Cegles (1998) believed the provision of support services a challenge to educators primarily because of the nature of DL education and the needs of DL students. Cohorts of WCET's (2003a) Learning Anytime Anywhere Partnerships project concurred since students who could not easily reach campus for class faced the same difficulty for support needs. The adequacy of support services, such as library services, for DL students tended to go unnoticed as long as the educational institution offered the service, yet a need existed to assess support services (Cain \& Lockee, 2002; Cooper, 2000; LaPadula, 2003; Sampson, 1999; Slade, 2001). Research lacked sufficient documentation about successful support systems although most colleges and universities offered them (Cain \& Lockee; Upcraft, Terenzini, \& Kruger, 2000; Visser \& Visser, 2000). 
Researchers described libraries as integral to learning by offering students those services that took into account their academic and research needs (Caspars \& Ragan, 2000; Simmonds \& Andaleeb, 2001). McLean (2000) suggested that library support for DL students remained a "low priority" for IHE yet various disciplines expected students to conduct research (p. 187). Barsun (2002) indicated that despite existing accreditation standards and suggested principles, institutions might have expected their DL students to utilize local library services instead of providing adequate academic services. Library services for DL students went relatively unnoticed by non-librarians (Cooper, 2000; Middleton \& Peacock, 2000). Library support services for DL students included digital reference and instructional services, remote access to online research tools, database and research tutorials, interlibrary loan, and document delivery.

Research showed the primary advantages of asynchronous reference service to DL students included serving students geographically remote from the campus, students employed hours when the library was open, students with physical limitations, students who preferred using electronic means of communicating, and students who opted to work from locations away from the library (Kenney, 2002; Staley, 1998). The principal advantages of asynchronous reference service for the library staff included additional time to address the student's question, an electronic means of sending information to the student, and a record of the response. The main advantage to the institution was the affordability of asynchronous reference service. While the advantages appeared ample, the adequacy of asynchronous reference service remained another area for evaluation.

One of the main disadvantages was communication and the inability to interpret visual and verbal cues from the student and to relay the same (Staley, 1998). Communication 
often appeared short and clipped between the librarian and the student leading one or the other to misinterpret the other's response. This concern translated to the online environment and affected the online community. Another major disadvantage was the time it took for a complete transaction to take place with messages going back and forth anywhere from two to five times (Staley, p. 20). Some students appeared indifferent about the time it takes for library staff to respond to an e-mail request due to the convenience in making the request.

Assessment helped to determine the impact of the service on the student. Webster's (2001) defined standards as principles for measurement, assessments as evaluations, and benchmarks as the "standard or reference by which others can be measured or judged" (p. 72). Stacy-Bates (2003) found that 102 (or 92.7\%) of the 110 ARL libraries studied offered asynchronous reference service (p. 61). Although determining standards and quality assessment had been a difficult task, researchers agreed that the profession needs a new reference model to address the expectations of digital reference service (Cram, 1993; Missingham, 2000; Whitlatch, 2001). The quality of service remained questionable without methods of assessing the service or without performance standards (Clarke \& Brinkley, 2000; Ferguson \& Bunge, 1997). Asynchronous reference assistance offered new areas for measurement and assessment useful in the establishment of norms (Carter \& Janes, 2000; Lessin, McGinnis, \& Bean, 2002).

An unobtrusive study was one way to assess the effectiveness and accuracy of reference services without the subjects' knowledge or consent (Cullen, 2002; Kaske \& Arnold, 2002; Tygett, Lawson, \& Weessies, 1996). Researchers have utilized and supported unobtrusive techniques for the past three decades (Crowley \& Childers, 1971; 
Dyson, 1999; Hernon \& McClure, 1986; Jirjees, 1981; Kaske \& Arnold; Saxton \& Richardson, 2002). The subjects conducted their daily activities without having to alter their professional habits thereby reducing possible bias (Novotny, 2001). Crowley and Childers supported the use of unobtrusive research to "increase the validity of the data as representative products of library information service" (p. 25). Whitlatch (2001) agreed that unobtrusive evaluation has a place in the digital realm.

The accuracy of reference responses was one way of measuring the effectiveness of reference service. Hernon and McClure (1986) concluded that library staff answers reference inquiries correctly only $55 \%$ of the time (p. 37). The $55 \%$, or half-right, rule emerged from this study and it has remained the benchmark most studies strived to surpass (Crowley, 1985; Plotnick, 1985). No matter what type of library undergoes evaluation, Ross and Dewdney (1998) stressed that success rates remained around the 5060\% mark (p. 151). Kaske and Arnold (2002) conducted a study of chat and e-mail questions. They found a 55\% accuracy rate for 133 chat sessions and a $60 \%$ accuracy rate for 107 e-mail inquiries (p. 1).

\section{Summary}

When IHE initiated DL course and program offerings, the ability for these institutions to provide adequate student support services remained uncertain. The nation's regional accrediting associations for colleges and universities required that support services provided to DL students be comparable to those offered to campus-based students. These same accrediting associations recommended the use of systematic and ongoing assessment of support services to assure continued quality and adequacy. Library services 
were just one of the support services recognized by accrediting associations. Library services available to DL students included, but were not limited to, digital reference and instructional services, remote access to online research tools, database and research tutorials, interlibrary loan, and document delivery.

The library profession already had documented problems in the provision of adequate and effective telephone and in-person reference service. Recognized challenges pointed toward the accuracy of reference responses and the treatment of patrons. Digital reference services exacerbated the challenge because existing literature did not adequately address the effectiveness of digital reference services for DL students, nor did it address how asynchronous library support services affected the academic experience of DL students. Standards had been developing, but libraries lacked standard criteria for offering quality digital reference services thereby impeding the ability to determine effective practices.

Chapter One addressed the problem, research questions, goals, definitions, assumptions, limitations, and relevance and significance. An overview of Florida's community college library system, a brief discussion of the advantages and disadvantages of DL, and a brief summary of unobtrusive testing techniques used for the past three decades to assess the effectiveness and accuracy of reference services supplemented this chapter. The need existed to study the adequacy of digital reference services given to DL students and the impact asynchronous communication had on reference services within the online environment. 


\section{Chapter 2}

\section{Review of the Literature}

\section{Introduction}

Chapter Two examined literature pertaining to reference service and the distance learning environment in an effort to survey existing research and to establish a framework for the study. The literature review included the following concepts and keywords: unobtrusive study, mystery shopper, customer relations management, reference accuracy, distance learning, distance education, online learning, online education, support services, library services, reference services, higher education, college, university, assessment, standards, benchmarks, online learning environment, online learning community, digital reference, and virtual reference. The researcher concluded Chapter Two with a discussion of barriers and issues, a summary of the known and the unknown, and contributions to the field.

\section{Accrediting Associations and Other Organizations}

To ensure adequate programs and courses in the Web environment, the nation's regional accrediting associations for colleges and universities stipulated that support services provided to DL students be comparable to those offered to campus-based students (Lebowitz, 1997). These same accrediting associations also mandated the use of 
systematic and ongoing assessment of support services to assure continued quality and adequacy. Accrediting associations strengthened the standards for DL library services because DL programs often failed or neglected to provide adequate library support services to students (Hufford, 2000, 2001; Lebowitz; Oregon University System, 1995; Pease \& Power, 1994; Southern Regional Education Board, 2002a). Gaide (2004a) considered library support services among the 10 best practices for student completion ( $\mathrm{p}$. 8). Among the regional accrediting associations, the New England Association of Schools and Colleges (2001) specifically mentioned digital reference services among other library services as "appropriate support" for DL students (p. 19). Edge and Edge (2000) agreed that accrediting associations were more closely evaluating equitable library services to DL students and they seek evidence supporting library services.

The Principles of Good Practice for Electronically Offered Academic Degree and Certificate Programs, the standards of the Western Cooperative for Educational Telecommunications (WCET) (1996), set the tenor for quality DL. The Principles, supported by the nation's regional accrediting associations, as well as groups like the Southern Regional Education Board's (SREB) Electronic Campus (SREC), guided DL in the areas of curriculum, mission, commitment, support services for students and faculty, assessment and more. The SREC applied the Principles of Good Practice in a number of ways, including assessment of quality. The SREC's application of the Principles mentioned access to learning resources and student services, but reference to the term, library, remained absent. The SREC also indicated a fall 2000 amendment to their version of the Principles through the Principles for Electronic Campus Library Services (Southern Regional Education Board, 2002b). This amendment, SREC's first, had not yet 
been published, leaving a void in library literature even though the SREB issued a subsequent policy report including library services and resources (Hamilton, 2000; SREB).

The policy report issued by the SREB's Distance Learning Policy Laboratory (2002b) asserted that higher education neglected to develop student support services while developing online courses and programs although support services affected student success. Sampling data used for this report indicated that library services ranked fourth of the 18 services mentioned as important for the success of DL students (p. 9). The report included library services as one of the key services in need of change for the DL environment and recommended that the Southeastern Library Network review the possibility of a regional library system. Furthermore, the report commended statewide initiatives, but encouraged regional services to support the educational requirements of DL students. A critical recommendation was to assess the core services for success.

The Institute for Higher Education Policy (2000) included the provision of library services, "resources, types of materials provided to students, response time to students, and student expectations," course structure, and student support benchmarks in its report, Quality on the Line: Benchmarks for Success in Internet-Based Distance Education (p. 9). Benchmarks remained subject to ongoing evaluative and assessment processes. The report noted high marks for library resources, and states, "Reference assistance to students is not neglected and some respondents suggested that a reference person should be available 24 hours a day, 7 days a week" (p. 18). 


\section{Impact of Digital Learning}

Campbell (1999) attributed the need to re-address the assessment of library's reference services to digital learning. The burgeoning use of the Internet for academic courses and programs during the 1990s cemented the need for digital library services. The Internet had played a significant part of the lives of students entering higher education. Due to their technological backgrounds, Campbell indicated they also exhibited a technological preference for seeking and receiving help from a librarian, as well as the types of resources they use. The supposition that libraries offered DL students necessary resources and services because of their established history of service was negated because most libraries neglected to conduct continuing evaluations of digital reference.

\section{Online Learning Community}

Researchers defined community as a group of individuals who come together through ongoing communication, shared activities, cultivated relationships, and a mutual history (Cothrel \& Williams, 1999; Jonassen, Peck, \& Wilson, 1999; Lock, 2002). Jonassen, Peck, and Wilson (1998) believed that online learning communities further emphasized the definition of community by offering a "means for learning within an atmosphere of trust, support, common goals, and respect for diversity" (p. 4). Lock suggested that an online learning community developed when relationships evolved through the application of "communication, collaboration, interaction and participation...the four cornerstones in a learning community framework" (p. 397). Kibbee, Ward, and Ma (2002) found that email contributed to the community as it had created profound changes in communication at the personal, academic, and corporate levels. Wills (2004) stressed that e-mail 
communication was a "new medium" with its "best practices" in the early development stages (p. 91).

Jones (2002) considered participation in the college community necessary for the growth of the student's social and academic relationships. Lorenzetti (2002b) found online learning communities reduced attrition. Palloff and Pratt (1999) advocated that communities facilitated online learning through both asynchronous and synchronous methods of interaction. Sacks (1996) quoted Harasim as saying, "Online education is evaporating all the old boundaries, the things that keep people apart...For the first time in history we can have many-to-many communication across time and space" (p. 178).

Lock (2002) included the institution and its supportive components as part of the online learning environment. Fritch and Mandernack (2001) suggested effective virtual relationships created social or long-term bonds. Dewald, Scholz-Crane, Booth, and Levine (2000) recognized the need for librarians to establish ways of reaching and assisting students in the online environment to develop an effective relationship. Bell and Levy (2004) introduced the concept of an emotionally supportive learning environment, fostering trust and encouragement, for the success of the students (p. 141). Laukenmann et al. (2003) conducted a study establishing a correlation between academic success and emotional well-being. Kochtanek and Hein (2000) introduced the concept of the "studentto-guest" relationship, and the librarian fell into this category. Baker and Field (2000) indicated that traditional services could not compete easily in today's online environment so librarians needed to build "client-oriented service" (p. 23). Asynchronous communication enhanced the librarian's ability to develop an online learning community with the student. 
E-mail offered users an excellent method for developing or sustaining relationships because of the ease in facilitating virtual social relationships and breaking down traditional hierarchies (Baron, 1998; Wills, 2004). Human communication required a method of expressing one's self. Tu and McIsaac (2002) examined computer-mediated communication to observe how it led to the development of the user's online social presence. In the digital environment, e-mail was just one of the ways that students had to develop a social presence or an academic presence. E-mail facilitated student interaction, as it remained available to students whenever they need assistance within the digital environment.

Researchers considered asynchronous communication more widely accepted by students since they were at ease within the e-mail environment (Frederiksen, Cummings, \& Ursin, 2004; Jensen \& Sih, 1995). According to Jones (2002), author of The Internet Goes to College, $72 \%$ of the college students $(n=2,054)$ surveyed check their e-mail on a daily basis, $66 \%$ indicated the use of two separate e-mail accounts, and $26 \%$ used IM every day; therefore, e-mail was both "practical and convenient" for them (pp. 2, 5, 10). Casado (2001) suggested that more students than not possessed an e-mail account. For library service at a distance, Bristow (1992) indicated a student preference for e-mail reference as opposed to telephone reference.

The interaction between the patron seeking information and the librarian assisting the patron represented another element in community development since reference service was not always limited to library resources alone (Fine, 1995). Archer and Cast (1999) indicated the human element critical to the "success of the reference transaction" (p. 39). Carr (1986) spoke years earlier about a bond that developed and continued between the 
student and the librarian even after the initial interaction. Goetsch (1995) stated that the connection between students and librarians was vital.

Nash (2000) quoted Janes as saying, "Digital reference will hook people up and create communities, understanding, and growth" (p. 2). Venkatalakshmi and Sonker (2002) believed that a personal relationship was possible between the librarian and the student in the digital environment. A study that included librarians at Connecticut College, Smith College, and Wesleyan University found that digital reference services contributed to personal contacts between the librarian and the student (Cheng, Nathanson, MacFarlane, Hansen, \& Berger, 2002). Dinwiddie and Lillard (2002) discussed a DL study conducted by librarians at Central Missouri State University where $63 \%$ of the survey respondents $(n=115)$ indicated strong support for library assistance via e-mail or telephone (p. 204). When asked if they experienced comfort working with the librarian, $40 \%$ strongly agreed and $29 \%$ agreed that they did (p. 207). Of this same group, $46 \%$ and $35 \%$ indicated that they would seek help from the librarian with forthcoming projects (p. 208).

Jensen and Sih (1995) shared results of a study conducted at the University of California in San Diego and the University of California, Berkeley that identified the value of asynchronous communication between the student and the librarian. Although the study dealt with the use of tutorials to help students learn about subscription databases, a second finding resulted. As part of the study, library educators used e-mail to share information with the students, and found that a relationship developed between students and library instructors. The librarians involved in the study indicated an increased number of communications, reference questions, and interlibrary loan requests because of asynchronous communication. 
Wheeler and Fournier (2001) described the value of asynchronous library support in a case study of Master of Continuing Education students at the University of Calgary. Students used e-mail as a method of contacting the librarian when they struggled with their research workload. The greatest benefit to using e-mail proved to be the ability of the librarian to develop a relationship with the student. This relationship allowed the librarian to introduce the library staff as affiliates in the students' online learning community.

Lawson, Lillard, Antrim, and Morgan (2000) studied the direct involvement of a librarian as a co-instructor in an online class. This arrangement enabled the librarian to communicate with students through course e-mail and bulletin boards. A library link, included on the course page, allowed the librarian to create a resource page related to course curriculum. The primary benefit proved to be the direct connection the librarian established with the students. Initially, students believed their questions "bothered" the librarian, but the authors found that $52 \%$ of the students networked with the librarian (p. 253). More than $74 \%$ of the students strongly agreed that the librarian's presence proved beneficial to their course experience. Seventy-one percent indicated comfort in dealing with a librarian assigned specifically to their course, and 91\% strongly agreed or agreed that they would seek the librarian's assistance in future classes (p. 254).

\section{Library Support Services for Distance Learning}

Good practice dictated that students taking DL classes warranted the same library services that campus-based students received. Recommendations as to what constituted good practice varied across the nation. Orientation resources, contact information, 
electronic resources, remote access, reference assistance, document delivery, online tutorials, and course reserve constituted the list of services and resources (Gaide, 2004b; Krauth \& Carbajal, 1999; Olsen, 2001). Lebowitz (1997) reported that research dealing with support services to DL students seldom mentioned the library's role, yet Archer and Cast (1999) believed librarians needed to reach the patron and their questions wherever they were.

Beagle (2000) found "only about 10\% [of published references] discussed library access or the use of library resources in the DL curriculum in anything more than a cursory way" (p. 367). Cother and Parnell (2002) found that many institutions provided DL students only a portion of library services available to campus-based students. FurstBowe and Dittman's (2001) study showed that adult females faced barriers in the support services area when they returned to higher education. Access to the academic library surfaced as one of the barriers leaving students concerned about their ability to complete their education.

Educators grasped the need to integrate student support services into DL; however, a void in the literature existed as to what services worked and why they worked (Rumble, 2000). When support services became expensive or appeared invisible, they became targets for budgetary reductions. Academia marginalized the value of library services and resources when they did not expect faculty to advocate their use within their courses, assess their quality, or promote their availability to DL students (Beagle, 2000). Assessments made them more visible to fiscal administrators (Lipow, 1999). The need existed for libraries to offer digital reference services to their students since many students faltered while conducting research for academic purposes. 


\section{Student Skills}

Due to the incalculable amount of online resources available to students, the need existed to build a relationship between the student and the librarian. Researchers and faculty often misjudged the research skills of college students by mistaking technical skills for research skills or by assuming that students can answer complex research questions (Goetsch, 1995; Kong, 1995). Harris (2004) identified four skills needed for academic assignments. They included "knowledge of online resources, written communication skills, research strategies, and online searching skills" (pp. 111-112). Goetsch found that a small percentage of students exhibited research proficiency; however, most students used research materials so sporadically that they did not adequately develop their skills. Carlson (2002) quoted Herring, a Winthrop University librarian, who stated, "They don't see the difference between proprietary databases and free information on the Internet" and they could not adequately judge the reliability of the source (p. 2). Moore (1989) identified access to content material as one method of supporting student learning.

Vishik (1999) and Borgman (1996) found that the student's inability to retrieve quality resources and their difficulty in navigating the online interface contributed to the need for mediated library support service. Jones (2002) indicated that results of a study of 2,054 college students showed that $73 \%$ of students surveyed use the Internet in lieu of library resources, yet the study indicated that half of the students surveyed did not find the Internet sufficient for their research needs (pp. 3, 5). Research validated librarians as a critical component in providing support to students in an asynchronous learning environment (Wheeler \& Fournier, 2001). Coffman (2002) suggested that students need 
professional assistance when they failed to find what they needed via a search engine. McClure (2004) concurred with the student's preference to use online resources; however, the challenge was to help the students find the balance. They also needed direction locating reliable and unbiased information, helping narrow a search to manageable results, or finding information at no cost that the Web offers for a fee. Digital reference service was available to students; however, its success had not been adequately measured.

\section{Communication}

While e-mail communication remained a critical concern for libraries offering asynchronous reference service, Roysdon and Elliott (1988) as well as Abels (1996), found a lack of research available on the subject. The lack of research might be attributed to the belief held by service providers that they had proficient communication skills (Salem, Balraj, \& Lilly, 2004). Lee (2004) agreed that the use of virtual communication techniques posed both "opportunities and barriers" to virtual reference service (p. 106). Larson (1990) suggested the development of a new model for dealing with students in the online environment. The historical model relied upon a face-to-face transaction or a telephone conversation. The electronic model incorporated the best practices of the traditional model with developing practices of the electronic model. Chandler (2001) and Farmer (2005) believed that good communication and interpersonal relations provided the skills necessary for reference librarians to operate in the DL environment. N. S. Moffitt $^{1}$ (personal communication, April 4, 2004) believed that the current generation had

\footnotetext{
${ }^{1}$ N. S. Moffitt was the director of the Naval General Library Program in Florida.
} 
its basis in the online environment and that was how they preferred to be served or how they preferred to communicate.

Asynchronous interchanges between the librarian and the student lost some of the spontaneity of a traditional reference interview. Cyberspace failed to translate verbal clues and body language leaving the librarian to discern the question without immediate and personal communication. Straw (2000) believed that the manner in which the librarian responded showed the student the value that the librarian placed on his question. Datta (1987) stressed the need to make all electronic communications more effective, as well as carefully and clearly worded. Effective communication reduced time spent clarifying the missive. The online environment called for natural language free from grammatical and spelling errors, but language that offered well-structured sentences.

Five different approaches to the asynchronous reference interview emerged through Abels' (1996) study. The piecemeal approach resulted in a large number of messages going back and forth between the patron and the librarian. With this approach, the librarian received the information from the student in small and disordered increments. The librarian then needed to make assumptions about the inquiry or to continue asking the student follow-up questions. The feedback approach called for the librarian peppering the patron with feedback even though the patron had not yet responded to the previous follow-up question. The bombardment approach showed the librarian inundating the patron with a barrage of questions. The questions appeared disjointed and frustrated the patron. The assumption approach showed the librarian responding to the inquiry without complete knowledge of the student's needs. This approach minimized the stream of email if the librarian made the correct conjecture about the patron's inquiry. 
The systematic approach called for structure and the use of forms. This method proved efficient and more successful to the patron than other methods. The form asked for personal information, the subject of the search, and constraints. White (2001) advised against collecting unnecessary information due to privacy issues. Constraints involved external factors, such as deadlines, cost, information delivery, and internal factors, such as reading skills and personal motivation (Abels, 1996, p. 353). Forms helped the librarian to draw the needed information from the student (Abels \& Liebscher, 1994). With a systematic approach, Abels found the inquiry answerable within three to five messages (p. 354). The problem, clarification of the question back and forth between the librarian and the patron, summary of the information need, and response to the patron constituted the transaction.

Shamel (2001) supported the use of forms to gather information needed to direct some of the communication between the student and the librarian. Carter and Janes (2000) examined 3,022 questions submitted to the Internet Public Library between January and March 1999, and they learned that $26 \%$ of the patrons used unstructured e-mail rather than the reference form (p. 253). The structured form allowed for questions that facilitated the response from the librarian. Ryer and Nebeker (1999) supported additional questions, such as intended use of the information, sources consulted, and keywords. Janes and Silverstein (2003) suggested that Web forms frequently ask the same questions, yet those questions that would be of immediate assistance to the librarian appeared to be omitted from the forms studied by this team. Omitted questions included the deadline for a response, sources already used by the patron, preferred method of delivery, and the nature of the request (i.e., assignment). 


\section{Philosophy of Reference Service}

Keith and Kohut (1998) indicated that libraries might or might not have a documented philosophy of reference service. Unwritten philosophies might have caused confusion. Staff might or might not have supported the library's philosophy. They may have instead followed their own beliefs about the provision of reference service. Rettig (1996) suggested that the librarian handle each circumstance separately regardless of the service philosophy. A universal philosophy of reference service did not exist.

Traditional philosophies involved the concepts of service, instruction, and information. Janes (2003) stated that “reference services don't exist because reference librarians exist and need something to do...They exist to serve the needs of our communities of users in the situations in which they find themselves" (p. 169). The librarian provided the user with the information requested, with instruction on locating the information independently, or a combination of both types of services (Lipow, 2003).

Lipow (2003) indicated that two types of users exist. One who wanted to be taught and one who wanted to receive the answer. Rettig (1996) cautioned about libraries that had one philosophy for reference and one philosophy for instruction since they often appeared to be opposite. One philosophy encouraged self-sufficient learners and one philosophy encouraged the provision of information (p. 9). Keith and Kohut (1998) believed that the two philosophies should be in accord.

The advent of digital reference introduced the need to review existing service philosophies supportive of distance education students (Watson, n.d.). Watson suggested a "shift of philosophy in library services from that of a library oriented basis to that which is grounded in user oriented concerns... where they institute new patterns of service 
delivery" (pp. 4-5). The literature reinforced the need to meet the expectations of the student and to provide outstanding service to the user, yet nearly half of the digital reference service providers limited their service to ready reference inquiries and excluded in-depth research questions (Dilevko, 2000; Janes, 2001; Zanin-Yost, 2004). Janes suggested that service philosophies should reflect the library's "mission, users, communities, and needs" (p. 182). Hernon and Altman (1996) supported a customeroriented philosophy of service likened to that of the pharmacist or the airline pilot who each must fulfill the expectations of the customer in an accurate and timely manner (p. 7).

\section{Successful Digital Reference Services}

Shamel (2001) cited the availability of digital reference service from the Library of Congress through its pilot Collaborative Digital Reference Service and its successor, QuestionPoint, the Department of Education through the Virtual Reference Desk Project, the Internet Public Library, and an increasing number of educational institutions. QuestionPoint, a joint effort between the Library of Congress and OCLC, included all types of U.S. libraries and served as another international collaborative reference effort (OCLC, 2003). The Virtual Reference Desk advocated digital reference services through AskA Librarian services, supportive development endeavors, the VRD Conference, and publications (Virtual Reference Desk, 2002). The Internet Public Library received about 1,000 questions each month with a response rate of $80 \%$ (Oder, 2003, p. 17). Since 1995, the Internet Public Library (2005) answered 50,926 reference inquiries (p. 6). The U.S. Department of Education's AskERIC question-answer service, discontinued in December 
2003, handled more than 335,000 inquiries and requests during the last 10 years (“AskERIC,” 2003, p. 1).

\section{Asynchronous Service Models}

While some national asynchronous models existed and flourished, institutional models were still in the development phase. Researchers offered various opinions on models for consideration. Archer and Cast (1999) suggested four models for asynchronous reference service. In the first model, one librarian responded to the questions with other librarians assisting when needed. The second model showed the head librarian distributing questions to the librarians in a triage system. The third model called for the establishment of a shared e-mail service answerable by the librarians assigned to the service. The fourth model used a bulletin board where librarians answer the questions as they log into the service.

Giannini (1999) detailed characteristics of a digital reference model. The characteristics addressed communication, skill, process, and evaluative factors. The collaborative communication between the librarian and the patron appeared to strengthen the remainder of the model. A relationship formed between the two as they worked toward the patron's goal. The librarian assisted the patron within a virtual environment where the style of the reference interview changed. The student benefited from the expertise of the librarian and expected to find that expertise even in a virtual environment. While the interaction became a learning opportunity for the student, an expert never failed to extract new information, processes, and sources from the interface. Finally, the model called for the librarian to work with the patron until the patron 
indicated satisfaction with the results or until the patron indicated a need that the librarian referred to a more expert contact.

Lankes' (1998) model consisted of five steps: receiving the question, assigning the question, answering the question, tracking repeat questions, and building a database of questions. All of the models involved a written policy detailing the service. Part of the service model included specifics about the clientele served. For example, asynchronous service offered through institutions of higher education most often assisted students, college employees, and local community members. The service generally excluded clients outside of the institution's service district. The service model defined the types of questions that patrons may submit asynchronously. The service often limited the patron to submitting questions that involved facts or short answers. By restricting the types of questions, the service limited the number of e-mail transactions that were transmitted from the patron to the librarian (Abels, 1996; Archer \& Cast, 1999). The model might have included information regarding turn around time and referrals. The models did not include details about assessment.

\section{Standards for Digital Reference Service}

Researchers determined that the need to develop standards for participation in the asynchronous reference environment existed (Digital Reference Education Initiative, 2004; “Facets of Quality,” 2003; Lankes, 1998; Schilling-Eccles \& Harzbecker, 1998; Wheeler \& Fournier, 2001). Success rates varied significantly with differences stemming from a variety of existing local standards or the lack of standards (Hansen, 2004; Lankes; Schilling-Eccles \& Harzbecker). Fullerton (2002) stressed the need for standards, 
especially those services that were operated through a consortium. Francouer and Ellis (2001) recommended the use of Reference and User Services Association (RUSA) and Reference and Adult Services Division standards for practice. Finding even an acceptable standard for benchmarking appeared difficult; however, some areas emerged as critical.

Sjolander and Sjolander (1995) suggested that those institutions involved in strategic planning document a mission statement that stipulated organizational or departmental goals. The library's philosophy of reference service need not have changed because of advances in technology; however, the manner of executing service might have changed (Pease \& Power, 1994). The provision of effective reference service was a valid goal for a library. Objectives assisted the employees in determining how to achieve specified goals. The evaluation of outcomes enabled the organization to determine whether the employees achieved the departmental goal. Sjolander and Sjolander believed reference policies included high quality service, evaluative statistics, and other factors attributable to analysis. Frederiksen (2002) believed that assessment helped "identify, measure, and evaluate" goals and objectives for the purpose of upcoming use (p. 260).

The International Federation of Library Associations and Institutions (IFLA) drafted guidelines for digital reference services (U.S. Library of Congress, 2002). The guidelines included a number of practices designed to offer a successful digital reference service. Identifying the primary clientele, determining service goals, establishing the types of questions that the service will address, staffing the service, training the participating staff, designing the user interface, instituting a privacy policy and adhering to other legal issues, assessing the service, and applying changes to improve the service were the 
primary areas of concern. The guidelines also included specifics for asynchronous service to provide the patron with a comprehensive response to his or her question.

McClure, Lankes, Gross, and Choltco-Devlin (2002) recommended courtesy, accuracy, patron satisfaction, repeat users, and awareness of service, cost, time, and access as standards for service (pp. 60-61). They found that initial benchmarks assisted in establishing existing performance to determine how to improve or identify acceptable levels of service. Hutchinson and Pye (2004) concurred with determining "targets for performance" for user satisfaction, quality, volume, repeat users, and other related measures (p. 1). Lankes $(1998,2002)$ reported 12 characteristics that distinguished acceptable digital reference service from unacceptable digital reference service. The list included "authoritative, accessible, fast, private, consistent with good reference practice, clear in user expectations, reviewed regularly, provides access to related information, noncommercial, publicized, instructive, and offers training to experts" (Lankes, Collins, \& Kasowitz, 2000, p. 71).

Stemper and Butler (2001) completed the list by stressing the need to document understandable explanations of what users could expect. Hernon (1996) included accuracy of answers as a primary measure of service quality to library patrons. Response time reduced student anxiety and prompted the student to the next step in the research process. Accessing services, personalizing the service, encouraging the student, developing effective communication, using clear language, providing a learning experience with each interaction, and sharing research strategies were some other recommended attributes (Lynch, 2002). Murfin's (1995) focused on the user's assessment of the service lending credence to Lankes, Gross, and McClure's (2003) suggestion that 
user satisfaction represented a standard for inclusion in the assessment of digital reference service.

\section{Policies for Digital Reference Services}

Policies stipulating criteria for asynchronous reference service were unique to other library policies, assimilated into general library policies, or did not exist. Criteria included the acceptable clientele, nondiscriminatory approach to service, mission statement, service points, level of service, instruction, turnaround time, evaluation of the service, types of evaluation, use of evaluations, and other points recommended by ALA's Reference and Adult Services Division (1994). White (2001) indicated that many libraries provided little information about the types of questions they would answer; however, most excluded detailed reference questions. Stringent policies might have accounted for the low quantity of e-mail inquiries (Garnsey \& Powell, 2000; Lipow, 1999; Novotny, 2001).

V. Munch ${ }^{2}$ (personal communication, April 14, 2004) viewed digital reference as inconsistent in its delivery. Researchers agreed that libraries needed to inform their patrons about the specifics of the service so the patron was not misled (Kern \& Gillie, 2004; Schilling-Eccles \& Harzbecker, 1998). Kern and Gillie stressed the need to consider the library's mission when establishing digital reference policies. Corrigan, Diamond, and Hill (2002) found that only 19\% of the 21 libraries responding to their survey had written policies in place (p. 9). Tibbo (1995) emphasized that policies helped ensure fair and ethical service to the users. ALA (2004c) recommended posting

\footnotetext{
${ }^{2} \mathrm{~V}$. Munch was the Coordinator of Information Literacy at the Metropolitan College of
} New York. 
information on the Web or other noticeable places for user awareness. Information should have included all the parameters for the service, including all associated policies and standards.

\section{Advantages of Asynchronous Reference Services}

Before the advent of the Internet, Crowley supported the use of the telephone as an asynchronous library service by stating, "In this day of expanding communication networks, it would be anachronistic for the library to expect inquirers to visit the library except when they chose to do so" (Crowley \& Childers, 1971, p. 58). Kresh (2000) believed that e-mail was emerging as the preferred method of communication. In 2000, more than 300 million e-mails were sent daily (Kresh, p. 2). The projected number of email messages expected to be sent in the United States in 2007 was 2,693.9 billion ("Web Traffic," 2004, p. 1). Librarians had been using e-mail as one of their main technological tools since the early 1990s (Tomer, 1994). Students relied on the expediency of e-mail (Jones, 2002). Stormont (2001) believed that students' comfort level with the Internet created an opportunity to reach them digitally.

Straw (2001) believed asynchronous reference services were beneficial to libraries as they expanded the reach of service into the community. Dee (2005) found asynchronous service critical to students, especially if the institution did not offer other types of digital reference. A survey of 706 University of Iowa students showed that $71.3 \%$ believed Web/e-mail reference service were more important than other library services (Dew, 2000, p. 121). Rice (1993) studied 458 e-mail users at six federal and private locations and learned that the group overwhelming supported e-mail for both asking and 
responding to inquiries (p. 451). Bushallow-Wilbur, DeVinney, and Whitcomb (1996) found that $58 \%$ of library patrons $(n=114)$ at the State University of New York preferred e-mail communication to telephone or in-person interactions (pp. 362, 367). A study of digital reference services conducted at San Diego community colleges showed the number of e-mail questions far exceeded the use of the 800 number, the live chat, and the e-mail chat services available to students (Shamel, 2001).

Student comfort appeared to be one of the major reasons for offering asynchronous digital reference service. Wills (2004) maintained that e-mail provided users with a safe venue for communication. The literature suggested that e-mail protected the user from unwanted attention and embarrassment, but it also facilitated communication (Baron, 1998; Jesudason, 2000; Lipow, 1999). Students with various personalities, inferiority complexes, learning styles, and diverse English language skills found comfort in the anonymity of asynchronous communication (McGranahan, 2005; Tenopir, 2004a; Wills, 2004). Fister (2002), as well as Haines and Grodzinski (1999), readily admitted that libraries intimidated students, so the ability to ask a question anonymously helped eliminate the barrier. Students asked more questions, especially those they considered silly or stupid (Haines \& Grodzinski; Ma \& Wright, 1998; Swope \& Katzer, 1972). Researchers also identified advantages for the more reticent, inhibited, or insecure student (Archer \& Cast, 1999; Bichelmeyer \& Kiggins, 1998; Bristow \& Buechley, 1995; Chakraborty \& Tuñón, 2002; Foley, 2002; Francouer \& Ellis, 2001; Hahn, 1997; Kochtanek \& Hein, 2000; Roysdon \& Elliott, 1988; Sarkodie-Mensah, 1997; Straw, 2000). McGranahan (2005) supported asynchronous use to eliminate problems with 
biased librarians. Bias might have occurred when librarians believed students possessed research skills at a higher level than they had.

Duff and Johnson (2001) suggested that the e-mail reference inquiry put the question in the user's voice enabling the librarian to determine better the level of skill of the patron. Tibbo (1995) stressed the value of e-mail in that it compelled individuals to think before they acted. Katz (2002) concurred and said that writing the reference question helped the student form a more detailed inquiry. Abels (1996) said one of the advantages was that e-mail appeared mostly unstructured. Roysdon and Elliott (1988) suggested that the use of electronic records reduced the number of errors that occurred when writing the response to a reference question thereby providing the student with a more comprehensible answer.

Bushallow-Wilbur, DeVinney, and Whitcomb (1996) cited convenience as one of the primary reasons for using asynchronous reference service. Schilling-Eccles and Harzbecker (1998) believed that e-mail was as functional for digital reference as it was convenient to both the student and the librarian. Researchers commended the service for the convenience to users, especially those who took DL classes because of time and geographic constraints (Garnsey \& Powell, 2000; Hahn, 1997; Johnson, 1999; Ryer \& Nebeker, 1999; Sproull \& Kiesler, 1986; Straw, 2000; Toraki, 2002). Tenopir (2004a) indicated that students preferred working from home during nights and weekends, so the virtual environment was ideal for them. Twidale and Nichols (1998) found that e-mail facilitated expedient communication between the student and the librarian.

Caspars and Ragan (2000), as well as Abels (1996), believed that e-mail communication was easier for students and they had access to e-mail service through 
home, employer, public library, or campus computers. Researchers indicated that one of the conveniences was the availability of the service 24 hours per day, seven days per week (Fishman, 1998; Haines \& Grodzinski, 1999; Schilling-Eccles \& Harzbecker, 1998; Sproull \& Kiesler, 1986; Stanley \& Lyandres, 2001). The ability to ask a question when the student thought of it was important to the student. Deming (2000) suggested that with e-mail students might hear from the librarian more frequently during regular business hours.

The librarians indicated a preference for e-mail since the students received results whether or not they were online and it eliminated telephone tag (Schilling-Eccles \& Harzbecker, 1998; Straw, 2001; Whitaker, 1989). The asynchronous approach enabled the librarian to spend more time on the question so the responses might have been more accurate (Roysdon \& Elliott, 1988). Ewing and Hauptman (1995) criticized the amount of time librarians spent assisting one user at the expense of another, so the ability to spend quality time on asynchronous reference queries addressed directly their concerns.

George (2002) identified e-mail as a method to include anyone with an e-mail address as part of an asynchronous community without the restriction of time. Johnston and Grusin (1995) stressed that asynchronous reference services provided students support when they needed it. Students saw librarians as technologically savvy (Garnsey \& Powell, 2000; Schilling-Eccles \& Harzbecker, 1998; Twidale \& Nichols, 1998). Research showed that asynchronous reference allowed both the librarian and the student time to think about the question and the subsequent wording of the response (Abels \& Liebscher, 1994; Barsun, 2000; Coffman, 2004; Dewald, Scholz-Crane, Booth, \& Levine, 2000; Janes, 2002c; Lipow, 1999; Roysdon \& Elliott, 1988; Schroeder \& Zarinnia, 1999; 
Tibbo, 1995; Williams, 2002). Janes and Silverstein (2003) aptly called this time an opportunity for the information to "percolate" (n.p.). Time was especially helpful for students who were not native speakers of English (Novotny, 2001).

Digital reference services supplemented existing services already in place (SchillingEccles \& Harzbecker, 1998). The ability to request assistance electronically reduced yet another barrier affecting students (Wills, 2004). Becker (1994) stressed that the librarian had the opportunity to foster a personalized approach with the student through technology. Daniel (2003) supported Becker by stating that an accommodating environment helped support the growth of a relationship between the student and the librarian. Wills said that e-mail provided a method of building a social environment for students and much like talking, e-mail helped build relationships.

The asynchronous environment also helped the library staff. Researchers stated that the questions could be collected together at one time for dissemination to the appropriate librarian (Stanley \& Lyandres, 2001; Stemper \& Butler, 2001). Literature considered this process the equivalent of an efficient reference triage (Gorry, 1998; Janes \& Silverstein, 2003; Kosmin, 1990). Pomerantz, Nicholson, Belanger, and Lankes (2004) believed that the function of triage got the question to the expert suited to answer it. Duff and Johnson (2001) suggested that the use of e-mail for reference enabled the librarian to interpret the question without it being filtered through another person. Henson and Tomajko (2000) stressed the ability for service providers to confer with one another when they encountered difficulties answering a question. Janes (2002c) indicated that the librarian was also able to contact the student for further clarification of the question. Tibbo (1995), Tenopir (2001), and Schloman (1993) said that the student received better information 
and there was less stress placed on the librarian. Tenopir further determined that the use of e-mail allowed for a more in-depth investigation of the question. Dee (2005) stated that of the 132 academic health science libraries studied, 80 libraries offered only asynchronous reference (p. 26). Some libraries also preferred e-mail reference to chat reference because of staffing concerns or low usage of chat service (Dee; Thomsett-Scott, 2004).

Lee (2004) endorsed digital reference services as an opportunity for the reference librarian to augment his or her skills. Digital reference skills were critical if the librarian was to meet the demands of the $21^{\text {st }}$ century student (p. 96). McGranahan (2005) also believed that librarians improved their own reference skills when they participated.

Literature identified the written record as an indication of the librarian's performance and it created a permanent record of the transaction for both the student and the library (Janes, 2002c; McGlamery \& Coffman, 2000; Schloman, 1993; Staley, 1998). Barsun (2000) believed that students recalled more information when it was text-based as opposed to verbally presented, so the permanent record was of value to the individual. Referrals were easier to deliver when a written record existed (Fishman, 1998). The capability also existed to build a database of common requests (Butkovich, 1988; Fishman, 1998). Kosmin (1990) said that a databank of frequently asked questions lightened the burden on librarians assigned to asynchronous service.

Henson and Tomajko (2000) found that the written record of the digital transaction enabled evaluation and training opportunities. Janes (2003), as well as Janes and Silverstein (2003), suggested the documentation of asynchronous reference responses enabled librarians to track reference performance for assessment purposes. Question 
types, response time, accuracy of responses, and phrasing of questions and answers lent themselves to quality judgments. Tenopir (2001) stated that the numbers were still low so the service could be further developed. Bushallow-Wilbur, DeVinney, and Whitcomb (1996) also discussed how the low volume of questions enabled the development of the service without affecting other library services.

With the DL environment, students might or might not have had physical contact with the librarian. Users who had heretofore been unable to access the physical library now had an opportunity to do so. D. P. Dillard ${ }^{3}$ (personal communication, April 10, 2004) suggested that students might use the convenience of asynchronous reference service whether they were in the library or not as questions may also come from within the library itself. Patrons were able to ask for assistance without the restrictions of place (Staley, 1998; Straw, 2001). Williams (2002) described asynchronous communication as practical within the DL environment as students benefited from the flexibility of time and place.

One of the primary advantages to asynchronous reference service was its cost. The cost for asynchronous service was negligible (Schilling-Eccles \& Harzbecker, 1998; Staley, 1998). In terms of technology, most librarians and students already had e-mail (Janes, 2003; Sloan, 1998b). In most cases, the provision of such service involved the addition of an e-mail contact and form to the library's homepage at a nominal cost (Bushallow-Wilbur, DeVinney, \& Whitcomb, 1996; Coffman, 2004).

E-mail appeared compatible with most online systems and the time it took to communicate back and forth was quite fast (Ferguson \& Bunge, 1997; George, 2002;

${ }^{3}$ D. P. Dillard was the moderator of the MedRef-L listserv and a reference librarian for Temple University Libraries. 
Schilling-Eccles \& Harzbecker, 1998). Technologically, e-mail used less bandwidth and had a more far-reaching connection (Bober \& Dennen, 2001). The librarian used technology to transmit information to the student through attachments and scanned files (Kosmin, 1990). The service saved time in terms of document delivery (Abels \& Liebscher, 1994). Deming (2000) supported the use of e-mail within the online environment since many librarians and students kept their e-mail active during the day. The availability of asynchronous reference service allowed the library to promote yet another technologically based service.

\section{Disadvantages of Asynchronous Reference Services}

Tomer (1994) indicated that underutilization of e-mail reference services continued into the 1990s. Fishman (1998) suggested that the slow move toward higher usage provided libraries time to determine policies, procedures, and assessment methods; however, they did not. The absence of a written policy affected the demands on library staff (Fishman). During the 1980s, most e-mail requested document delivery of articles (Howard \& Jankowski, 1986). Summey and Fisk (2003), as well as Coffman and McGlamery (2000), added that many students remained unaware of DL library services as many studies showed this an underutilized service.

Response time appeared to be one of the negative points about asynchronous e-mail reference (Janes, 2002c, 2003; Philip, 1997; Schilling-Eccles \& Harzbecker, 1998). Abels and Liebscher (1994) believed that response time affected usage of the service. Email was written at all times of the day and night. Students often believed that the messages should be answered as soon as the send button was hit. Coffman (2004) 
suggested that librarians improved the turnaround time between transactions in order to increase usage.

Tenopir (2001) believed that responses to electronic inquiries were time consuming for librarians. Tyckoson (1999) found that asynchronous interaction lacked the immediacy that the reference interview provided synchronously. Kanuka, Collett, and Caswell (2002) believed that some librarians sent responses to asynchronous questions in a less than timely manner because they forgot that an actual person awaited a reply. Bell and Levy (2004) believed "it can be difficult to see [digital] learners as whole people" because their requests for information were done without the benefit of a physical or an auditory connection (p. 143).

By the time, the librarian had the information needed to assist the student, the student might not need the information or the student had changed the direction of his research (Abels, 1996). Crouch and Montecino (1997) described a student's anxiety when responses took longer than expected although Hara and Kling (1999) found that students appeared indifferent to the time of day and expected immediate responses to their e-mail inquiries. A lengthy transaction from the librarian might have discouraged the student, but Abels believed a librarian should be able to complete an electronic transaction within three to five messages (p. 354). The volley of messages proved difficult when responsibilities rotated among reference staff (Fishman, 1998; Hahn, 1997; Staley, 1998). The volley also reduced the ability to conduct a productive reference interview (Abels; Abels \& Liebscher, 1994; Coffman \& McGlamery, 2000; Fishman, 1998; Foley, 2002; McGlamery \& Coffman, 2000; Tomer, 1994; Toraki, 2002). 
The inherent problems with technology reduced the effectiveness of the service during its early years (Hahn, 1997; Philip, 1997). Technological incompatibilities appeared less problematic than they once were; however, it was important to recognize them (Abels, 2000). One of the earlier disadvantages was the student's inability to access a computer and e-mail. While most students possessed and utilized both of these items, some still did not (Burke, 1996; Janes, 2003). Weissman (2002) noticed that fewer email addresses failed to send, a trend that affected asynchronous reference during the initial years.

Research indicated that the location of the service affected its success and its availability to students. Casey, Sochrin, and Race (2002) believed that a poor location on the library's Web page impeded notice and access by students. Kawakami (2002) indicated that a buried link equaled service failure. Dee (2005) mentioned access from a variety of locations problematic, i.e., direct e-mail, Web page link, form, or hidden link.

The student's technology skills influenced his or her ability to access or input information (Foster, Evans, \& Lott, 1997; Janes, 2003). Technical skills, such as keyboarding and attaching files, slowed down the process, especially for adult students for whom technology was a new skill or for those visually impaired (Crouch \& Montecino, 1997; Dewald, Scholz-Crane, Booth, \& Levine, 2000; Donnelly, 1995; Foley, 2002; Harrington \& Spindle, 1993; Whyte, 1995; Zafeiriou, Nunes, \& Ford, 2001). The ability to convey an adequate message also depended on the student's writing skills (Staley, 1998). Wills (2004) said, "We need to keep in mind that e-mail takes place between human beings who constantly struggle to understand and be understood" (p. 89). 
Sloan (2003) said that inaccurate, poorly written, and inadequate responses from librarians affected digital reference services. The librarian who sent an e-mail with misspelled words or grammatical errors negatively affected the reputation of the professional librarian and the library itself (Abels, 1996; Fishman, 1998). Hansen (2004) saw the opportunity for abuse to occur when librarians provided incorrect information, personal opinions, personal analysis, and more. While an increasing number of questions were being asked of digital reference services, the accuracy of the information provided to the user was not screened (Lamolinara \& Grunke, 1998). Librarians might have also ignored the student's request if the question appeared too difficult for the librarian to answer. Referrals created problems for the student, too, if the librarian did not first verify the availability of the resource on the patron's behalf (Fishman). Nilsen (2004) suggested that unmonitored referrals acted as a method of purging the patron request form the inbox.

Wills (2004) indicated that librarians might have opposed the use of e-mail for reference service since a paper trail developed which may be used later in time. Written records stood to be detrimental to the library staff if they remained accountable for the responses made to patrons (Fishman, 1998; Philip, 1997). Janes (2003) also described librarians who preferred transitory queries since their professional skills came under scrutiny. They might also have restricted the types of questions students were able to ask. They accepted uncomplicated reference inquiries, but they found it problematic to accept detailed reference questions despite expectations for equivalent services within the digital environment (Schilling-Eccles \& Harzbecker, 1998). 
If the student lacked research skills or literacy skills, he or she might have encountered additional obstacles once the librarian provided a response (Janes, 2003). Staley (1998) said that the student's reading skills might have negatively affected the usability of a response. Research skills, a necessary part of higher education, were more difficult to explain and to understand in the virtual environment. Wheeler and Fournier (2001) learned that students relied on the librarian before investigating resources independently and often asked questions that other departments handled more effectively.

Communication problems hindered the relationship between the student and the librarian. Foley (2002) found the asynchronous environment impersonal and that the impersonal nature negatively influenced the relationship between the student and the librarian. The barriers were exacerbated in the asynchronous environment (Ammentorp \& Hummelshoj, 2001). Non-traditional students, such as adult students and students for who English was not their first language, might have also experienced additional barriers in the asynchronous environment due to their own challenges in the classroom.

Philip (1997) found the inability to conduct a reference interview a disadvantage to providing digital reference services. The patron's question might have been misunderstood or the patron might have provided inadequate information. De Groote (2005) intimated that the type of information needed by the student determined what type of service mode was warranted. Librarians needed to express flexibility in the virtual environment. The inability to read facial expressions or hear verbal clues reduced the effectiveness of asynchronous service (Burge, 1994; Eastmond, 1995; Fishman, 1998; Hill \& Stahr, 2003; Kochtanek \& Hein, 2000; Tibbo, 1995). 
White (1981) found that the loss of face-to-face interaction removed opportunities for clarification. Druckman, Rozelle, and Baxter (1982) studied nonverbal communication and learned "that up to $93 \%$ of the intent of communication is conveyed by facial expression and tone of voice" (p. 85). Other researchers found that the lack of visual and verbal clues hampered a workable relationship between the student and the librarian (Abels \& Liebscher, 1994; Harris, 2004; Janes, 2002c; Sproull \& Kiesler, 1986; Staley, 1998). Fritch and Mandernack (2001) indicated that the in-person interaction could not be easily replicated in the virtual environment. The ability to do so was a value-added ability.

Students contributed to the breakdown in communication when they failed to respond to the librarian's questions or response (Harris, 2004; Sloan, 2003). Janes (2003) said that the student's lack of social skills stressed the tenuous relationship between the librarian and the user. He called the student a "disappearing questioner" (Janes, 2002c, p. 5). This lack of feedback prevented the librarian from continuing with a request asking for clarifying information or it prevented the librarian from knowing whether the student found the response usable (Carter \& Janes, 2000; Janes, 2002c; Janes \& Silverstein, 2003).

The student was not the only individual at fault for the breakdown in communication. The tone of the librarian's message affected the student (Lamolinara \& Grunke, 1998; Sloan, 2003). Nilsen (2004) suggested that the tone of the e-mail caused a higher or lower level of satisfaction with the patron. Tone influenced the impression that the patron believed the librarian had about the question and the use of the librarian's time. 
Staley (1998) said that short responses appeared abrupt to the patron. The behavior of the librarian contributed to the success or the failure of the reference transaction.

Philip (1997) identified the loss of patron confidentiality problematic. Gross, McClure, Hodges, Graham, and Lankes (2001) believed that asynchronous reference services compromised a patron's confidentiality. Janes and Silverstein (2003) agreed that the library collected far more information about a virtual patron than librarians did with face-to-face transactions.

\section{Staffing of Digital Reference Service}

ALA (2004c) indicated knowledgeable, interested, and available trained personnel best provide the service. While the volume of asynchronous questions was increasing, the number of questions received remained consistently low at academic institutions with some usage logged at 23-60 questions per month (Corrigan, Diamond, \& Hill, 2002, p. 9). Corrigan, Diamond, and Hill's survey indicated that $67 \%$ of their survey respondents utilized librarians and staff to answer e-mail reference inquiries while the other $33 \%$ used one librarian (p. 9). Gross, McClure, Hodges, Graham, and Lankes (2001) found digital reference generally handled by the same staff handling in-person reference desk. Of those institutions utilizing professionals and staff to answer questions, paraprofessionals accounted for only $14 \%$ of the total and $5 \%$ of the graduate students (p. 9). Staffing appeared to be limited mainly to librarians (Goetsch, Sowers, \& Todd, 1999).

Stemper and Butler (2001) advised that not all reference staff had the skills to handle digital reference. Basic and advanced training facilitated the ability of librarians to provide virtual training to their patrons (Lankes, Janes, Smith, \& Finneran, 2004; 
Riechel, 1986; Straw, 2001). Wasik (1999) stipulated that a comprehensive training initiative prepared the reference staff for effective service. Gupta (2001) recommended training to best review new services and the expectations of clientele.

The 2001 survey of the Association of Southeastern Research Libraries (ASERL) (2003) indicated training was provided to more than half of its 32 respondents. These libraries provided full day training and additional support through supplementary electronic resources, such as Web sites, distribution lists, and software. Communication within the online environment constituted a portion of the training. Tenopir (1998) recommended that librarians become familiar with as many resources as possible, as well as the organization of electronic resources. Tibbo (1995) stressed that librarians also needed good listening, reading, and writing skills.

Roy (1995) suggested that training and educational preparation affected the accuracy of a response. Dewdney, Marshall, and Tiamiyu (1991) recommended training for subject areas that librarians believed were weak spots in their provision of service. Dilevko (2001) indicated that something as easy as reading the newspaper improved reference accuracy. Gers and Seward (1985) learned that when librarians requested feedback regarding their response, their accuracy rates increased from $52 \%$ to $76 \%$ (p. 34). The simple question, "Does this answer your question?" let the patron make the final decision regarding their satisfaction with the response. Weech (1984) mentioned peer review to evaluate reference responses. 


\section{Reference Service}

Green (1876) recorded the first mention of reference service to library patrons. He recommended four functions that librarians should offer as part of their reference service, including library instruction, research assistance, reader's advisory, and marketing library services to the public. Green further emphasized a personal relationship developing between the librarian and the patron (Tyckoson, 2003). Librarians offered reference services in a variety of formats, including traditional, telephone, asynchronous, synchronous, and telefacsimile (McGlamery \& Coffman, 2000). Although reference services evolved slowly from Green's time until now, the advent of technology resulted in critical changes. Kosmin (1990) found that technology helped to advance the "scholarly information flow" (p. 217). The use of technological tools like e-mail and the Internet guided the changes leading to digital reference services.

A 2001 survey of the ASERL (2003) reported that 32 of the 44 members responding to the survey indicated the availability of e-mail reference service (p. 3). Of this group, $9 \%$ began offering service in the latter part of the 1980 s, $31 \%$ started in the early part of the 1990 s, $47 \%$ initiated service in the latter part of the 1990 s, and $13 \%$ failed to provide a start date (p. 3). Henson and Tomajko (2000) indicated that digital reference services started sometime in the late 1980s with the majority of services starting in the early 1990 s (pp. 113, 115). The University of Maryland Health Services Library initiated its electronic mail service in 1984, Lehigh University in 1986, and Indiana University in 1987 (Bristow, 1992, p. 631; Roysdon \& Elliott, 1988, p. 82; Wiese \& Borgendale, 1986). Straw (2001) found that libraries embraced technological changes brought by the Internet, and incorporated applications into their operations by the end of the 1990s. 
Ware's (2004) survey of reference resulted in 748 responses from member librarians from the Association of College and Research Libraries (ACRL), RUSA, Library Administration and Management Association, Public Library Association, the LIBREF listserv, and the Dig_Ref listserv. Survey results showed that 99\%, or 741 respondents, indicated that answering reference inquires and search strategy questions was a reference transaction (p. 1). Seventy-nine percent, or 509 respondents, recommended measuring patron satisfaction with reference services. Fifty-nine percent, or 386 respondents, recommended measuring the time spent on each question. Fifty-nine percent, or 384 respondents, recommended measuring the accuracy of responses. Fifty-seven percent, or 372 respondents, recommended evaluating the resources used by librarians to answer questions (p. 2).

\section{Reference Interview}

Bushallow-Wilbur, DeVinney, and Whitcomb (1996) specified that most questions acceptable in the digital environment involved little discussion. The librarian needed to ask at least one question of the patron about the inquiry for a reference interview to have taken place (Nilsen, 2004). Gers and Seward (1985) observed that the use of follow-up questions improved responses. Straw (2000) suggested that digital reference interviews appeared less formal than person-to-person interviews. They provided the librarian more opportunities to promote library services to the student or to follow-up with the student. Cannon (2002) stressed the need for reference librarians to modify the manner in which they handled reference interviews, including the cultivation of the relationship with the online patron. He called these "value-added" opportunities (p. 37). 
Ross and Dewdney (1998) offered strategies to avoid "negative closure" of reference interviews before an acceptable answer was provided to the patron. Referrals to useless resources accounted for reference failure. Ross and Dewdney noticed $44 \%$ of the library transactions they evaluated failed due to unmonitored referrals (p. 154). Murfin and Bunge (1984) considered unchecked referrals to be failures in reference service causing significant drops in success. Proposing that the patron accept information that had not been requested caused reference failure. This action sometimes happened when the librarian asked the patron to change his or her topic because the question appeared too difficult to handle. Reference failure also occurred when the librarian ignored the patron's request. Ronan (2003) recommended techniques such as adding links, using encouraging communication, personalizing the communication, expressing interest, using expert resources or assistance to answer the inquiry, and introducing searching techniques to the student (p. 45).

\section{Reference Questions}

One cannot assume that the type of question was unimportant to the user. Coffman and Saxton (1999) suggested that little research had been done on the types of questions asked and the best way to answer these questions. Arnold and Kaske (2002) reviewed the characteristic types of unique and non-unique questions answered during a reference transaction. Unique questions related to local information about the library or the institution. Non-unique questions required basic catalog, database, or manual searches to locate the answer. They included directional, ready reference, specific, and reference questions as categories documented by Katz (2002, p. 16), as well as holdings and policy 
questions. They documented 225 chat sessions broken down by ready reference at $36 \%$, policy at $31 \%$, directional at $15 \%$, and specific search at $12 \%$ (pp. 11, 16). Straw $(2004)$ examined 594 chat sessions and learned that 181 questions were reference in nature and 201 related to a "known resource or service" (pp. 93, 96).

Duff and Johnson (2001) reviewed Grogan's schema, which included eight different types of questions. Limited help questions included administrative and directional, author and title, and fact type questions. The open-ended questions included material finding, mutable questions, research questions, residual inquiries, and unanswerable questions (Duff \& Johnson, p. 49). They adapted Grogan's schema and created their own. Administrative and directional involved questions related to policies and procedures of the library or the IHE. Fact-finding questions asked for the answer to the question, but the patron did not want to seek the answer directly. Material finding questions asked whether a library had resources on a particular topic. Specific form questions asked for specific record types in the library. Known item was already knowing what was needed, but checking to see if it was available in the library. Service requests sought copying or interlibrary loan. Consultation invited recommendations for information. User education requests sought help in getting started on research. Although the study was performed on e-mail reference questions ( $\mathrm{n}=392$ ), Duff and Johnson indicated 27\% were service requests, $17 \%$ were material finding, $13 \%$ were user education, $11 \%$ were directional, $10 \%$ were consultation, $10 \%$ were seeking fact finding questions, $8 \%$ were specific forms, and $4 \%$ were for known items (p. 54).

Kibbee, Ward, and Ma (2002) found that their study of digital reference questions $(\mathrm{n}=604)$ at the University of Illinois at Urbana-Champaign showed users seeking 
particular library resources (33\%), library information (31\%), subject search (20\%), ready reference (9\%), problem with technology $(5 \%)$, and questions about virtual reference (1\%) (p. 33). Horn and Kjaer (2000) analyzed statistics associated with virtual reference service at the University of California, Irvine. They learned that $76 \%$ of the questions are reference and 17\% were access (p. 139). Kawakami (2002) noted the types of questions include specific items, library and its services, how to locate, ready reference, technical, and questions about service (p. 29).

The 2001 ASERL (2003) survey indicated that $41 \%$ of questions received were library or institution-specific inquiries, 38\% were ready reference, $29 \%$ were research in nature, and 13\% related to other question types (p. 8). Marsteller and Mizzy (2003) reviewed the literature and suggested that ready reference questions were better served by digital service than the lengthier reference inquiries. They shared results of a study of Carnegie Mellon's synchronous digital reference service and found that of the 865 transcripts reviewed, $32 \%$ were technical in nature, $17 \%$ were for librarian communication, $17 \%$ addressed directional type questions, $14 \%$ were known item searches, $10 \%$ were ready reference, and 10\% were reference (p. 156). Sears (2001) reported 44\% for directional type, $22 \%$ for ready reference, and 33\% for reference (n.p.).

Sears' (2001) study of chat reference services at the Auburn University Libraries took place during spring 2001. The study lasted about four months and included 153 questions (p. 8). Nearly half of the questions were ready reference and one-third was policy related. Sears categorized the questions using Katz' (2002) definitions. These definitions included ready reference, specific search, research, policy and procedural, and directional (p. 16). Bunge (1999) found that the types of questions $(n=48)$ asked in academic libraries varied 
with the top five responses as follows: known item at 14\%, information on a topic at $14 \%$, facts at $12.4 \%$, material format search at $14 \%$, and short answer at $9.7 \%$ (p. 131).

Hill, Madarash-Hill, and Bich (2003) assessed results of a survey and determined that of 37 respondents, $28 \%$ of questions asked requested help with library resources, $19 \%$ required a short response, $13 \%$ warranted a referral to databases, $10 \%$ called for a referral to another department within the library, $7 \%$ needed a referral to Web sources, $7 \%$ required in-depth assistance, $4 \%$ asked additional questions, $3 \%$ needed direction to another institutional service, $1 \%$ requested document delivery, and the library staff asked $1 \%$ to visit the library for in-person assistance (pp. 4, 7-8).

An ARL (1999) study showed that 51\% of the 76 responding libraries indicated they would have recognized all questions, but in some cases, the response given to the student might have been as brief as recommending an in-person consultation (p. 2). Forty-one percent of this group said they accepted only ready reference questions for factual information (p. 2). When these types of statistics were reported, the limitation of digital reference to ready reference appeared to fail the user.

Kosmin's (1990) survey of 4,500 transactions showed more than 57\% were catalog checks, literature searches, and reference queries (p. 221). Garnsey and Powell (2000) studied 329 public libraries and found that $33 \%$ of the questions were ready reference, $25 \%$ were research in nature, and 18\% were genealogy questions (pp. 247, 250). Powell and Bradigan (2001) discussed a study conducted at Ohio State University where the questions fell into nine categories: access to databases and journals, verification of references, health information, assistance with databases, directory information, library holdings, library services, course information, and statistical questions (pp. 174-175). 
Hodges (2002) documented ready reference at 63\%, known item searches at 29\%, and research type questions at $8 \%(\mathrm{p} .164)$.

Sloan (2002) discussed a review of 877 sessions conducted during the fall 2001. Of the questions asked, 30\% were research questions, $20 \%$ were for specific journal needs, $14 \%$ were for ready reference, $9 \%$ were for known items, $9 \%$ were technical questions, $8 \%$ were library policy questions, $6 \%$ were other questions, and $4 \%$ were for citation requests, (p. 34). Marsteller and Mitzy (2003) analyzed 425 chat sessions and indicated that $33 \%$ were policy questions, $28 \%$ were known item questions, $19 \%$ were ready reference, and 19\% were reference questions (p. 157).

The type of fact-based reference questions routinely asked during assessment studies represented only a portion of question types users posed (Richardson, 2002). Fricke and Fallis (2004) identified ready reference as the easiest type of question to answer correctly. Richardson also found some questions distinctive to a library's community, complex in nature, or irrelevant to contemporary society. Whitlatch (1989) found only $7.5 \%$ of the questions asked at the San Jose State Library proved to be factual; however, most digital inquires were restricted to ready reference (p. 184).

\section{Reference Responses}

The decision to respond with an answer versus sources depended on administrators of the service (Carter \& Janes, 2000). The question itself lent some insight into the type of response warranted; however, the patron might have specified the preferred type of response. Cother and Parnell (2002) found that DL librarians provided more resources and services to DL students, especially if they believed students were unable to access the 
resources independently. Sloan (1998a) suggested that the librarian determined the best way to provide an answer or to discuss the question with the student. Wyer (1930) suggested librarians provide the answers as well as showing the patron how to search for information. The librarian then bore the onus for searching and locating a current, accurate answer that relied on his or her expertise and judgment.

Stahl (1998) suggested four parts to an e-mail reference question. The parts were restating the patron's question, providing a short answer, offering additional resources, and closing with a favorable mention of the responding institution. Fritch and Mandernack (2001) suggested that reference transactions appeared to be more like lessons instructing patrons in the use of resources, the types of information available, directing users in their searching, aiding them in information retrieval, and helping them to best present their information (pp. 294-295).

\section{Accuracy}

The accuracy of the reference response appeared to be one of the critical factors studied; however, the determination of what was accurate remained. The scoring scale for reference questions fluctuated from study to study with many studies resulting in the 55\% rule determined by Hernon and McClure (1986, p. 37). Ewing and Hauptman (1995) noted that the probability for error existed with human involvement and studies indicated low accuracy rates for even fact-finding questions. Studies limited to factual questions appeared to be the common methodology used to test accuracy of reference service (Cram, 1993). 
Parus (1996) believed that patrons expected an accurate, concise, and immediate response (pp. 5-6). Burge (2001) suggested that patrons determined satisfaction through “just in time, task-relevant, respectful and accurate" service (pp. 8-9). While researchers stressed user satisfaction as a primary factor in the success of a reference transaction, Pierce (1984) suggested that users could not always differentiate an accurate response from an inaccurate one.

According to ALA's Standards and Guidelines Committee of the Reference and Adult Services Division, accuracy was an expectation regardless of the difficulty of the inquiry (Roy, 1995). Cronin (2000) stated that minor errors might not be life threatening; however, they did have a "corrosive effect upon public perceptions of professional competence" (p. 44). Venkatalakshmi and Sonker (2002) supported the need for accurate responses and they stressed the "right information to the right user at the right time" ( $\mathrm{p}$. 1). Elzy, Nourie, Lancaster, and Joseph (1991) advocated for accurate answers, not just an answer acceptable by the patron. Hill, Madarash-Hill, and Bich (2003) found inadequate service related to incomplete responses lacking in possibilities or failing in the provision of an accurate, comprehensive response.

Childers determined that a correct answer was a "criterion of quality" (Crowley \& Childers, 1971, p. 80). Dewdney and Ross (1994) stated that "accuracy of the answer recommends itself as a measure to researchers because it is one of those reliable variables (like "precision" and "recall") that are not dependent upon subjective judgments" (p. 219). Hill, Madarash-Hill, and Bich (2003) indicated that assessment of reference responses was a subjective measure, in part, to incorrect answers and, in part, to inadequate service to the patron. 
Studies conducted on the accuracy of reference responses showed accuracy rates hovered in the $50 \%-60 \%$ range, but rates rarely went as high as $75 \%$. McClure, Lankes, Gross, and Choltco-Devlin (2002) reviewed library literature and found that the "correct fill rate" happened less often than one anticipated a quality service should offer (p. 19). The earliest known studies receiving attention were those studies done by Crowley and Childers. Crowley's study showed a 54\% accuracy rate while Childers' showed a 55\%64\% accuracy rate (Crowley \& Childers, 1971, p. 91). Childers' literature review showed Enoch Pratt Free Library with a 63.9\% success rate (Crowley \& Childers, p. 93). The study conducted by King and Berry (1973) showed a 60\% accuracy figure.

Weech and Goldhor (1982) found an accuracy rate of 70\% when they conducted an unobtrusive study and $85 \%$ when they conducted an obtrusive study of five public libraries in Illinois (p. 305). Hernon and McClure (1983) conducted accuracy studies using 17 academic libraries in the northeast and southwest. They used 20 questions and delivered half by phone and half in person. Accuracy in the northeast was $49 \%$ and $20 \%$ in the southwest for a combined accuracy rate of $37 \%$ (p. 305). ALA's Reference and User Services Division tracked challenging reference questions that appeared in a column called "The Exchange" and learned that an analysis conducted in 1985 showed that they answered only 47.25\% questions (Anderson, 2003, p. ix). Human error accounted for a number of inaccuracies in answering reference inquiries.

Gers and Seward (1985) discussed a study dealing with the accuracy of reference inquiries. They asked 40 questions of 60 libraries within 22 library systems for a total of 2,400 inquiries. The results of the study showed that $38.2 \%$ provided a correct answer with the source information and $16.7 \%$ provided a correct answer without the source 
information for a 55\% accuracy rating (pp. 32-33). The authors considered these two responses to be levels one and two; however, another level of service providing referrals gained no points for accuracy.

Hernon and McClure's (1986) study of the accuracy of reference responses determined that library staff answered 50\%-60\% of questions asked correctly (p. 37). They said that these questions had a low difficulty rating. Woodard (1989) studied the accuracy of responses to reference questions asked at the University of Illinois Library, and learned that nonprofessional staff answered $62 \%$ of its questions correctly (p. 455). Lewis (1995) indicated that $40 \%-50 \%$ of the time librarians failed to locate materials even if their library owned the item (p. 11). Ross and Dewdney (1998) documented a success rate within the 50\%-60\% range (p. 151). Bunge (1999) cited success scores for 42 public libraries studied at $60 \%$ and 48 academic libraries at 56\% (p. 125). Librarians from both of these groups believed themselves more successful than reported. Studies conducted since the Hernon and McClure (1986) study validated the results of their study (Campbell, 1999).

Dilevko (2000) sought to replicate the Hernon and McClure (1983) study done of U.S. government document depository reference services using Canadian depository libraries. Of the 488 reference questions asked, only $29.3 \%$ had a complete response, $13.1 \%$ had partially complete responses, $20.1 \%$ referred questions elsewhere, and $37.5 \%$ of the questions had an incorrect answer or no answer at all (p. 45). Another study mentioned by Dilevko showed inaccuracies in answering questions that appeared in the local newspaper. This accuracy rate was improvable through daily reading of the news. 
The Washington State Library completed a three-year project aimed at improving the accuracy of reference service provided to its citizens (Stillwell \& Moore, 2002). The training component of the project accentuated the value of preparation to improve services. Transform, Inc. conducted the training, and participating libraries indicated an improvement over the $55 \%$ rule. A six-month accuracy assessment conducted by Transform offered a sample of telephone transactions that provided the basis for Washington's accuracy benchmark. Using an unobtrusive telephone survey technique, patrons received accurate and complete answers only $58.4 \%$ of the time (p. 24). Following both an extensive training session and a follow-up study, comprehensive and accurate responses improved to $72 \%$ (p. 25$)$.

Kwon (2006) studied the delivery of virtual reference through the Broward County library system in Florida. The study considered 420 chat transactions. Although this study focused on referrals initiated through chat reference, accuracy statistics for the entire service were included in the results. Kwon found that $56.4 \%$ of the questions were answered completely, $4.8 \%$ of the questions were answered partially or were not answered, $29 \%$ of the questions were referred, and $9.8 \%$ of the questions had "problematic endings" (p. 10). Circulation inquiries accounted for the high referral rate.

Hernon and McClure (1986) indicated that accuracy decreased when the providers made referrals to internal or external sources or when they mishandled search strategies and question negotiation. Richardson (1999b) attributed 30 years of accuracy statistics at the 50\% mark to poor reference negotiation skills (p. 211). Dilevko (2000) attributed the poor results to library size, time of day, reference desk activity, manner in which the question was asked, the content of the questions, and electronic skills. Dilevko (2000) 
and Jackson (2002) agreed that the self-development of skills led to improved accuracy statistics.

\section{Referrals}

Hernon and McClure (1986) believed that a referral was a reference failure. They found that $16 \%$ of the 390 questions asked during their study were referred to an internal source (p. 39). Childers (1980) identified two types of referrals. The first was called steering, which was when a librarian directed the patron to another source without further mediation (p. 928). The second was called referring in which the librarian facilitated or initiated contact on behalf of the patron (p. 928). Ross and Dewdney (1998) shared a similar view of referrals. They identified referrals as unmonitored or verified (p. 161). The unmonitored referral transferred the onus of the contact and verification to the patron. The librarian predetermined if the verified referral would be of some use to the patron. Gebhard (1997) recommended librarians verify referrals for the most comprehensive and successful service to patrons. Kwon (2006) recognized that referrals might affect user satisfaction within the virtual environment.

\section{User Satisfaction}

Schweitzer (2003) defined customer relations management (CRM) as any type of interaction between the company and the customer. Cocheo, Harris, and Kirk (2003) included in-person, telephone, and electronic means of communication as methods of interaction. CRM aimed to retain a valued customer base of customers who repeated their business because they were satisfied with the company's services and products. Library 
staff could have learned more about strengthening their customer base since the Internet failed to provide many students with the quality resources they needed for their academic needs (O’Sullivan \& Scott, 2000). Many corporations offered customers the opportunity to seek assistance through a call center. Librarians needed to follow the literature on company-based call centers as they often employed scalable technology or techniques facilitating a satisfactory interaction between the customer and the employee (McGlamery \& Coffman, 2000). CRM offered students the value-added care and interactive service needed in their online learning community (West, 2001).

User satisfaction was another critical factor assessed to determine quality reference service. Riechel (1986) believed that a satisfied patron tended to establish repeat business when needed. Although users could not always determine the accuracy of the answer they received, they could articulate their satisfaction with the service received and the utility of the information given (Richardson, 2002). White (1999b) stressed that patrons needed reassurance that the service given to them was excellent. Tenopir (1998) believed that today's students have heightened expectations for service. In most cases, they expected the Internet to answer all of their questions. This "impression" intimated that anyone could find the information sought regardless of skills (White, 1999a, p. 265). Dilevko (2000) suggested that patrons expected a high level of service and reference questions enabled librarians to display their skills to patrons.

Nilsen (2004) suggested user dissatisfaction stemmed from omission of any type of reference interview, failure to verify a referral, and abandoning the patron without closing the transaction. Murfin (1995) attributed user opinion to the patron's satisfaction or dissatisfaction with the information received. Furthermore, Murfin stated that the 
librarian's behavior, including follow-up, search assistance, and rephrasing the initial question, helped to improve satisfactory reference responses. Librarians lost satisfaction ratings when they did not give total assistance in providing the answer.

Adult students determined satisfaction through service that was "just in time, taskrelevant, respectful and accurate" (Burge, 2001, pp. 8-9). Academic librarians referred students to sources more often than providing them the answers, possibly reducing the success rate determined by the student (Bunge, 1990). Carter and Janes (2000) studied over 3,000 questions asked of the Internet Public Library in 1999, and they learned that $33 \%$ of the time the patron wanted the answer and $33 \%$ of the time they wanted the source (pp. 251, 257). Sloan (2003) reviewed user satisfaction with the virtual reference service offered to the North Suburban Library System in Illinois. He learned that more than $17 \%$ of the 1,004 users surveyed complained about receiving guidance rather than a direct answer to the question, waiting for a response, being misunderstood by the librarian, and receiving an inaccurate answer from the librarian (p. 13).

Fine (1995) attributed failed reference transactions to the inability of the reference librarian to ferret the actual question and the inability of the patron to advise the librarian that the help was appreciated, but the results were not helpful. An ongoing Dig_Ref listserv discussion regarding librarian literacy offered a variety of comments about the competencies of librarians and the ability to serve the patron. S. Weissman ${ }^{4}$ (personal communication, November 15,2004$)$ wrote, "I suppose in any library, for any staff, it boils down to: make the information universe work for the patron."

\footnotetext{
${ }^{4} \mathrm{~S}$. Weissman was the Electronic Reference Co-Manager for the Morris County Library
} in New Jersey. 


\section{Librarian Behavior}

ALA's (2004b) Guidelines for Behavioral Performance of Reference and Information

Service Providers identified the behavior of librarians as contributory to quality reference service. The Guidelines delineated approachability, interest, listening and inquiring, searching, and follow-up skills as critical to the success of a provider customer interaction, and the encouragement of patrons to use the service again. The provider should have possessed the Professional Competencies for Reference and User Services Librarians as stipulated by ALA (2003). Among the more notable competencies were responsiveness, critical thinking and analysis, knowledge base, communication and outreach, and collaboration with users, colleagues, and other professionals. Consistency was maintained through routine evaluation and assessment measures.

The Digital Reference Education Initiative (DREI) (2004) posted a rubric of core competencies recommended for digital reference providers. These competencies considered three levels. The beginning level was the skills focus, the intermediate level was the conceptual focus, and the advanced level was the administration focus (p. 1). Each level was designed to build upon the other. The DREI anticipated the rubric to be used to educate digital reference practitioners. Tenopir (2004b) viewed these skills as a method of conducting staff development for both practitioners and for novices.

Richardson (2002) and Straw (2001) suggested the librarian's reference skills, knowledge of the library's collection, and educational accomplishments as factors for determining quality reference service. A chasm existed between the digital information environment and the role librarians traditionally played in connecting the patron to the information (Campbell, 1999). Campbell suggested that librarians lost valuable time 
developing a new model of reference service between the advent of Internet in the layperson's world and the librarian's acceptance of the impact of the Internet on libraries. A recent discussion on the Dig_Ref listserv about librarian literacy offered a variety of comments, including one from S. Haman ${ }^{5}$ (personal communication, November 15, 2004) who wrote, "Many of us have great skills, but no comfort level with technology...we're probably about the same level as a cross section of our users." Another posted message from K. Schneider ${ }^{6}$ (personal communication, November 15, 2004) stated, "Librarians who were "reluctant to change" were in the wrong profession, as are librarians who were "unable" to change."

Dyson (1992) believed behavior, knowledge of the resources, and skills in ferreting information were the three primary abilities needed to conduct a successful reference transaction. Fine (1995) said, "While librarians are aware that there is some predictability about the ways users behave in libraries; they are less likely to be aware that there is predictability in their own behavior as well" (p. 17). Cram (1993) detailed the critical behaviors essential to librarians. The primary behavior was verification of the student's question before answering it. Follow-up questions, open questions, knowledge of the library's collection, clarification of the student's question, and interest were the other behaviors mentioned by Cram.

Dewdney (1986) indicated that patron questions, however simple they may appear, often developed into an inquiry that was more complex. The librarian failed due to reliance on the "ill-formed query" without questioning the patron (Ross, Nilsen, \& Dewdney, 2002, p. 22). The emphasis on attitude constituting a critical part of the

${ }^{5} \mathrm{~S}$. Haman was a digital reference librarian for California's AskNow service.

${ }^{6} \mathrm{~K}$. Schneider was the director of the Librarians' Index to the Internet. 
transaction appeared to have disappeared from the transaction. Dilevko (2000) declared that traditional reference staff might have lacked the searching skills needed for digital reference.

Hernon and Altman (1996) held focus group sessions on the subject of virtual library services. Participants emphasized accuracy, relevance, appropriateness of fit, currency of content, ease of access, communication skills, and determining the needs of the patron as crucial. Lankes (2004) suggested that the expenditure of institutional resources warranted consideration of the strengths and weaknesses of digital reference services. Dilevko (2000) stressed that the success rate of librarians signaled opportunities to improve service to the patron.

Schwartz and Eakin (1986) recommended an evaluation of attitude and behavior. Some of the standards for communication overlapped with the librarians' behavior. Tibbo (1995) identified remote interaction as a form of communication. The tone of the interaction might have been different from face-to-face or telephone exchanges. The librarian needed to infuse the human touch into their interaction with the patron. L. Kortz $^{7}$ (personal communication, June 9, 2004) said, "In my experience, most libraries tend to be rather rigid and rule-bound and unfortunately this translates into the online environment. Also, many librarians see their interactions with people as a simple question and answer." Ward and Kern (2004) reminded librarians that they could discourage patrons as easily online as they could in person.

\footnotetext{
${ }^{7}$ L. Kortz was the Virtual Services Librarian at the New Jersey City University Library.
} 
B. $\operatorname{Sloan}^{8}$ (personal communication, April 22, 2004) said that his review of chat reference showed that $21.43 \%$ of users $(\mathrm{n}=182)$ complained about the attitude of the virtual librarian. He suggested examples of better ways to phrase questions so as not to be perceived as rude or unfriendly. Characteristics included a friendly manner, approachable style, enthusiasm to help, nonjudgmental responses, encouraging comments, and willingness to refer questions that the staff cannot answer. Straw (2000) recommended that librarians treat the virtual transaction with the same attention as the face-to-face transaction.

Radford and Thompson (2004) discussed results of a random sampling done of 245 synchronous chat sessions stemming from a statewide service in Maryland (p. 2). They identified numerous communication techniques as harmful to building a relationship with the patron. They included programmed answers, scolding the patron, disregarding the question, misinterpreting the question, unsuitable response, neglecting to support or encourage the patron, failing to notice the patron's attempt at humor, and using foul language (Radford \& Thompson, p. 2).

\section{Turnaround Time}

Corrigan, Diamond, and Hill's (2002) survey of the academic libraries in Louisiana showed that asynchronous reference service posted no specific hours of service because the format enabled service whenever staffing allowed. They determined that $50 \%$ of the responding libraries $(\mathrm{n}=21)$ answered inquiries as they arrived, $45 \%$ during irregular times, and $5 \%$ at scheduled times (p. 9). Response time for 35\% of the inquiries occurred

\footnotetext{
${ }^{8}$ B. Sloan was the Senior Library Information Systems Consultant for the University of Illinois Office for Planning and Budgeting in Urbana.
} 
within 24 hours, $12 \%$ between 24 and 72 hours, and 6\% between 12 and 24 hours (p. 9). Of the remaining respondents, $45 \%$ documented a less than 3-hour response rate (p. 9). Most inquiries arrived during normal afternoon and evening hours of operation (p. 10).

Hill, Madarash-Hill, and Bich (2003) indicated that it took 15 minutes to answer 11\% of incoming questions, 60 minutes to answer $40 \%, 2-3$ hours to answer $58 \%$, and 24 hours for $2 \%$ (p. 6). When surveyed, $76 \%$ of the patrons scored "very satisfied" with the service and $16 \%$ scored "satisfied." Only $8 \%$ of the respondents scored "not satisfied" and indicated the reason as timeliness of response (p. 7).

G. Remelts ${ }^{9}$ (personal communication, March 5, 2004) asked why libraries would offer a service with a turnaround time of days versus hours. Dissatisfaction was certain to be the result. The library should have posted a policy with its service indicating the turnaround time. Howard and Jankowski (1986) documented one of the earliest electronic mail services and it indicated a response time of 24 hours (p. 41). Ryer and Nebeker (1999) indicated 24-28 hours appeared to be the norm (p. 28). Bonham (1987) indicated a response within 24 hours and a check of the e-mail twice per day (p. 538). Sloan (1998b) suggested set times to check for e-mail and specific limits on turnaround. Casey, Sochrin, and Race (2002) reported Central Michigan University responded to its digital reference inquiries within 24 hours (p. 122).

Horn and Kjaer (2000) analyzed statistics associated with the University of California, Irvine, virtual reference service. They learned that Monday through Wednesday were the busiest times for the service with the most popular times occurring during regular business hours (p. 139). Turnaround time was an average of seven hours

${ }^{9}$ G. Remelts was the Library Director for the Calvin College and Calvin Theological Seminary in Michigan. 
with some questions answered within 4 minutes and some within 22.5 hours (p. 140). Librarians at North Carolina State University Libraries documented e-mail reference response time at 2.5 hours during normal operating hours (Anderson, Boyer, \& Ciccone, 2000, p. 3). The 2001 ASERL (2003) study showed that $28 \%$ of its 32 respondents responded to e-mail inquiries within 24 hours, more than 53\% reported 24 hours to next day response, and 9\% responded with a 24-48 hour window (pp. 4-5).

Kawakami (2002) noted numerous e-mails arrive during business hours of 10:00 a.m. through 4:00 p.m. (p. 28). Bushallow-Wilbur, DeVinney, and Whitcomb (1996) found that patrons submitted most of their questions during the library's regular business hours allowing for a response from the library staff during the same or the next business day if the patron submitted the question late in the day. Stanley and Lyandres (2001) discussed the results of the Bushallow-Wilbur study and agreed that users submitted most questions during the course of the day. They believed the user preferred the anonymity of electronic communication as well as the ease of asking questions from their preferred location.

\section{Assessment of Library Service}

Cother and Parnell (2002) believed the competition for students in the DL environment contributed to the need for institutions to develop "quality assurance measures and indicators" for student support services, including digital library services (p. 151). Concerns about the adequacy and equality of digital library services arose from accrediting associations, library associations, and the academic community. Assessment of institutional effectiveness should occur on a regular basis, especially if effectiveness influenced funding and performance (Campbell, 1999; Lankes, Collins, \& Kasowitz, 
2000). McClure, Lankes, Gross, and Choltco-Devlin (2002) also recommended assessment to develop quality service especially since DL created new opportunities for service. Burgin and Hansel (1990) believed libraries committed to improving performance needed to evaluate their services. Pomerantz, Luo, and McClure (in press) stressed the need to evaluate reference in order to identify inadequacies in the provision of service.

Gross, McClure, Hodges, Graham, and Lankes (2001) found services started without much planning. With libraries at various stages of offering digital reference service, officially accepted standards and policies did not exist (Fullerton, 2002). Standards for service appeared widespread and varied (Lankes, 1998; Schilling-Eccles \& Harzbecker, 1998). The interest existed, although efforts influencing the development of national standards appeared lacking. Frederiksen (2002) acknowledged that inadequate documentation existed to assess digital library services. The instruments for assessment, the areas of assessment, and the frequency of assessment appeared unclear.

Frank (1998) indicated that questions were not tracked or assessed; however, the number of questions asked by e-mail had increased. Corrigan, Diamond, and Hill's (2002) survey of academic libraries in Louisiana found that most of the 21 respondents neglected to assess asynchronous services, and they did not have procedures in place to do so (p. 9). Only 19\% of this same group had written policies for asynchronous library services (p. 9). Janes (2002a) surveyed reference librarians and learned that only $9 \%$ reported routine evaluations of their digital reference services (p. 552).

Acceptable benchmarks appeared intangible, making assessments more difficult to perform. If asynchronous reference services did not offer the quality expected by library 
administration, the need existed to conduct an analysis of the service to pinpoint problem areas. Jones (2003) concurred with strategic planning methodologies; however, the advice to libraries was to document statistics, measures, policies, and other materials used to develop or assess digital reference for accreditation purposes.

Bundy (2000) found measures valuable for benchmarking and advocacy endeavors. Libraries cannot base success of a service on the number of transactions (Cram, 1993). Roy (1995) stressed the need to measure accuracy because it helped librarians know the possibilities of service as well as acceptable standards. Aluri (1993) suggested that the evaluation of reference service facilitated the provision of more accurate responses in a timely and thoughtful manner. These measures and indicators included standards, statistics, observing performance, and analyzing the results to confirm confidence in the product and to identify areas for improvement.

Green and Peach (2003) suggested that the literature dealing with evaluation of reference services appeared to follow three categories. The 55\% school followed unobtrusive study techniques to denote the accuracy of the response. Accuracy in answering reference questions appeared to be a measure of effective service. Hernon and McClure (1986) were most often associated with the 55\% rule (p. 37). The interpersonal communication model concentrated primarily on the manner in which a librarian approached and assisted a patron rather than on the accuracy of the question so that the patron returned to the librarian for assistance. Durrance (1989) followed the interpersonal communication model. The third category referred to the patron's satisfaction with the entire transaction. Bunge (1990) and Murfin (1995) used the Wisconsin-Ohio Reference Evaluation Program (WOREP) instrument to measure user satisfaction. 
Bunge provided 12 years of historical data based on the results of the Wisconsin-Ohio Reference Evaluation Program (Stalker \& Murfin, 1996). His study showed that 7,013 questions asked in 74 academic libraries in the United States had a success rate of 57\% (p. 423). The Program established $60 \%$ as the criterion for quality service. The success rate varied depending on the resources used, the expertise of the reference staff, the availability of online resources, and the amount of time devoted to the inquiry. Bunge (1990) found a range of 52\%-69\% in terms of success in answering difficult questions (p. 46). Overall, he found a range from $48 \%-80 \%$ with most scores in the mid-50s (p. 46). Since the 55\% benchmark exists, Hults (1992) believed it should be the baseline for quality. Bourne (1965) discussed the $90 \%$ library since perfection is generally unachievable (p. 93).

Whitlatch (2004) conducted an unobtrusive study of in-person, telephone, and e-mail reference service during the spring 2004. Each of the 23 graduate students participating in the study asked the same question at three different academic libraries, but using a different method at each library. The study was designed to monitor the success and failure of the three modes of service delivery. Whitlatch found that the e-mail questions resulted in a success rate of $69.6 \%$ with failures attributed to non-response, unmonitored referrals, and questions deemed out of scope for e-mail service (pp. 1-2). Quality affected the consistency and dependability of the service; therefore, Whitlatch supported evaluation for all methods of delivery.

Dyson (1999) reviewed four unobtrusive surveys conducted in Maryland public libraries between 1983 and 1994. Maryland used the unobtrusive study method developed by Crowley and Childers (1971). The surveys addressed both the accuracy of the answer 
and the behavior of the librarian in answering questions. Based on the results of the first survey, subsequent training took place in Maryland. After the second unobtrusive survey, Dyson identified improvements in service directly related to training with accuracy ultimately reaching a $77 \%$ accuracy level by the fourth survey.

Due to the training program and positive outcomes from Dyson's (1999) second survey, Model Reference Behaviors resulted. Two other surveys occurred in Maryland using the Model Reference Behaviors, and the study showed a correlation between the Model and quality service. The Model Reference Behaviors, covering such topics as approachability, comfort, interest, listening, inquiring, searching, informing, and following-up, followed a standards-based approach to offering quality service (Dyson). Other libraries located within the United States adopted the Model based on the results Dyson reported.

Saxton and Richardson's study, conducted in 12 California public libraries, indicated that $90 \%$ of the questions drawn from 9,274 inquiries had an accurate response or an accurate referral (Richardson, 2002, p. 42; Whisner, 2003). They also believed the questions did not represent authentic reference questions and represented too small of a sample size. Saxton and Richardson (2002) used hierarchical linear modeling to conduct their analysis of the dependent and independent variables measured by their study. The dependent variables included outcomes such as utility, patron satisfaction, and accuracy (Whisner, p. 298). The independent variables included inputs such as the characteristics of the question, the librarian's behavior, the patron, librarians' response to a survey, and characteristics of individual libraries (Whisner, p. 298). The level of difficulty affected the accuracy response, and a group of independent librarian panelists scored the accuracy 
of the responses. Based on results of studies such as Saxton and Richardson, Dyson's (1999), and Hernon and McClure's (1986) standards for service appeared beneficial for measuring the effectiveness of asynchronous reference services.

White (1999b) included quality control and evaluation as part of the framework for the analysis of electronic question and answer services. The framework presented 18 categories by which a service can be assessed. The quality control category included its maintenance, inadequate quality, and a review process (p. 7). The evaluation category encompassed the availability of formal evaluation, a process of evaluation, the occurrence of evaluations, the performances measured by the evaluation process (i.e., accuracy, timeliness, user satisfaction), further interaction with the user, meeting the goals of the service, and statistics for the service (p. 7).

ServQual, another attempt at a national measure, considered reliability, assurance, empathy, responsiveness, and tangibles for assessment purposes (Novotny, 2001, pp. 116-117). Reliability addressed dependability and accuracy of responses. Assurance considered the attitude conveyed by the library staff to provide credible service. Empathy spoke to the considerate manner displayed by the library staff towards the end user. Responsiveness to the end user included timeliness.

Janes $(2000,2002 a)$ recommended that assessment instruments include accuracy, response time, instructional orientation, user satisfaction, self-evaluations, description of response, and queries that fell outside of posted policies. Martin, Crowley, and Shaughnessy (1969) indicated the need to review attitude and helpfulness. Roberts and Sergesketter (1992) studied Fortune 1000 executives and learned that accuracy, then the timeliness of the response, were the primary indicators of service quality (p. 18). 
McClure and Lopata (1996) suggested that extensiveness, efficiency, service quality, impact, and usefulness became areas for measurement (pp. 113-114). Extensiveness of service took into account the number and type of end users. Efficiency translated into the economics of the service. Service quality measured the satisfaction of the end user, as well as the provision of the service. Impact assessed how the service made a difference to the end user. Usefulness gauged the value of the service to the end user. Katz (2001) stressed the need to provide answers, not just referrals, within the online environment. Lipow (1999) found timeliness and accuracy critical to the success of reference service. Understandable communication conducted within a timely manner was critical to the success of the transaction (Lagace \& McClennen, 1998).

\section{Measurements of Reference Service}

The history of the measurement of reference service spanned at least 70 years with the first identified mention being Guerrier's (1936) article on measuring reference service. Although more than 1,000 documented studies existed, researchers who gained widespread recognition for their work on the assessment of reference services included Bunge (1990, 1999), Childers and Crowley (1971), Hernon and McClure (1986), Saxton and Richardson (2002), and Weech and Goldhor (1982). Their research supported the need to measure reference service; however, the standards and factors varied. Cullen (2002) maintained that standards have not emerged for "accuracy, fill rate, quality, service and satisfaction" (p. 30). Harrington and Spindle (1993) recommended the use of evaluations to determine effectiveness and to provide opportunities for revision and 
implementation of new or existing processes. Evaluation was the method to maintain quality control.

Hirko, Foley, and Pitney (2004) advocated the use of the Virtual Evaluation Toolkit. The Toolkit included evaluations for three levels of virtual reference. The first level addressed a checklist for the virtual transaction, a checklist to assess the Web site, a survey review, and a checklist to review virtual reference policies (p. 3). The second level involved a log analysis of the Web server and a self-evaluation conducted by the digital reference provider (p. 4). The third level included a peer assessment component, analysis of transcripts, focus group sessions, and a method of conducting usability testing (p. 5). The main purpose was to increase utilization of the service while maintaining customer satisfaction.

Gross, McClure, Hodges, Graham, and Lankes (2001) reviewed the employment of user surveys, unobtrusive testing, log analysis, content analysis, usability, focus groups, descriptive statistics, peer review, staff performance review, librarian discussion groups, technical analysis of hardware/software, cost benefit analysis, and cost effectiveness measures for digital reference assessment (p. 10). Thomsett-Scott (2004) addressed the increased use of focus groups to assess reference service. The use of transcripts showed librarians that they could provide exceptional service (Tennant, 2003). Gross, McClure, Hodges, Graham, and Lankes believed that despite the lack of formal measures, the written reference transaction could be used for quality control assessments through administrative or peer evaluations. Accuracy, completeness, tone, grammar, and the use of opening and closing remarks were areas for review (p. 6). Descriptive statistics appeared to be the more widely used type according to the authors. Gross, McClure, and 
Lankes (2001) believed accuracy, efficiency, and effectiveness had not been evaluated. The need existed for metrics and baseline data (p. 19).

McClure and Lankes (2001) proposed measurements for establishing benchmarks in digital reference. These measures included outcome measures, which are quality measures detailing accuracy, interaction, instruction, and user level; process measures, which are effectiveness and efficiency of process detailing access, timeliness, clarity, staff training, review and evaluation, privacy, publicity, and percentage of questions answered (service extensiveness); economic measures, which address the cost and effectiveness of providing the service; and user satisfaction which addresses users' experience and satisfaction with the service included many of the indicators mentioned in the other measures (p. 22).

Wasik (1999) considered the evaluation of digital reference service necessary to offer continued quality. Ward (2004) based evaluation of reference questions on a scale of five: complete, mostly complete, mostly incomplete, incomplete, and referral (p. 49). Wasik and Silverstein (2001) presented a session on the quality of digital reference and they offered a set of criteria for judgment. Criteria for assessing quality depended on the accuracy of a comprehensive response, the clarity of the answer, the suitability of the response based on the patron's age or level of understanding, the sources cited as evidence for the answer, and the instructive assistance provided to the user.

Tyckoson (2001) and Richardson (2002) supported numerous values in answering reference questions; however, accuracy was the "most common value" (p. 188). It stood in time as a measurement of reference success. Tyckoson added thoroughness to the accuracy value since it complemented the completeness or comprehensiveness of an 
answer, thereby strengthening its accuracy value. Authority was Tyckoson's final value as it balanced both the accuracy and thoroughness values and facilitated the timeliness value.

In $1999,87 \%(n=65)$ of the 76 responding libraries indicated they had not conducted evaluations of the service (Goetsch, Sowers, \& Todd, 1999, p. 3). One of the areas for evaluative consideration was the interaction between the patron and the librarian. The patron chose to use the service because he or she had difficulty locating the information, decided to save time, or could not determine the value of the information they were given. Accuracy of responses and user satisfaction were just two methods of evaluating service outcomes. Unobtrusive techniques were widely used to assess the accuracy of responses.

Wheeler and Fournier (2001) stressed the following points for adequate reference services. The inclusion of a greeting and a closing signature signified personal interest. Stress on the validity of the question sent a message to the student that the librarian valued their time. An informal style of communication that was clear, concise, explanatory, and free of jargon was understandable to most individuals (p. 430).

Closing comments pointed toward an interest in the patron's success and an opportunity to address inadequate responses (Richardson, 1999a). Management of Reference Services Committee, Management and Operation of Public Services Section of Reference and Adult Services Division indicated interviewing, consistency, courtesy, attention to patron, accurate responses, source, caution with negative responses, etc. (ALA, 1994). Staley (1998) urged librarians to restate the question, ask clarifying 
questions, use one e-mail box, include policies, procedures, policies, and send follow-up e-mail.

University at Albany Libraries (2002) stipulated attitude, accuracy of answers with the proviso that the librarian relied on existing data, no bias, equity of service to all users, and privacy. The staff mediated referrals before being sent to the patron. Accuracy protocol included answering the question using an authoritative source (Fricke \& Fallis, 2004). A completely accurate response was entirely correct. A partially correct response was mostly correct. A partially inaccurate response had little or no correct information. A completely incorrect response had no correct information. Fricke and Fallis believed the accuracy and comprehensiveness of an answer went hand-in-hand.

Weech and Goldhor (1982) included staff skills and library collection as part of the assessment equation. Hernon and McClure's (1987a) discussion of "half right" reference responses tied in with human and physical resources (pp. 3-4). Problematic reference interviews, failure to refer questions when unable to answer them, and inadequate collections or an inadequate grasp of the collection affected an accurate response valued by the patron.

Roy (1995) indicated that reference questions were measurable quantitatively when graded on a scale offering levels of correctness for service. The correct-answer fill rate was the ratio of questions answered correctly divided by questions asked (Hernon, 1987). Elzy, Nourie, Lancaster, and Joseph (1991) indicated a scoring method ranging from 15 points to 0 points that addressed the accuracy portion of their study. They also devised an attitude scale. Although a high mark in attitude related to a high mark in accuracy, the 
opposite was not true. A low attitude scale did not necessarily mean a low accuracy rate (p. 461).

Paskoff (1991) rated the answers to an unobtrusive telephone survey as accurate, inaccurate, referral, or no answer/refusal (p. 183). Childers (1980) employed a scale with correct, mostly correct, non-answers, steering, wrong, mostly wrong, and escalator queries. Escalator queries began as broad requests, but they became more specific as the librarian interviewed the patron (Gers \& Bolin, 1999). He grouped some of the categories together; however, he established referrals as non-answers. The patron might or might not have followed up with the referral.

Thomas (2000) used some of the following factors to measure effectiveness: interest, communication, encouragement, rephrasing question, open ended questions, language, unbiased, search strategy, explanation of actions, asked if additional information was needed, asked for feedback from student, and encouraged repeat visit (p. 6).

McClure, Lankes, Gross, and Choltco-Devlin (2002) suggested assessments that analyzed areas, such as, turnaround time, the total of unanswered questions from both librarian and patron, the type of question received by the digital reference service, sources used to answer the questions, the personalized manner in which the question was answered, and the extent that digital reference staff went to answer the question for the patron.

\section{Unobtrusive Testing}

Unobtrusive studies emerged as the predominant method for evaluating reference services. Unobtrusive testing constituted a "legitimate means of data collection and 
provides insights that are not easily obtained otherwise" (Hernon \& McClure, 1986, p. 37). An unobtrusive study tested people or services without their knowledge or consent under seemingly normal conditions (Kaske \& Arnold, 2002; Thomas, 2000). Hubbertz (2005) and Hébert (1994) found the use of unobtrusive testing allowed for the observation of service situations enabling a natural view of the encounter and results. This technique generated data not easily garnered from other methods of study.

Tygett, Lawson, and Weessies (1996) discussed a study conducted at Western Electric's Hawthorne plant during the late 1920s and early 1930s. The Hawthorne Effect indicated that an individual's behavior changes when he or she knew that someone was studying it. Pomerantz, Luo, and McClure (in press) conducted an unobtrusive study of virtual reference in 2004 . They believed that an obtrusive test would trigger the Hawthorne Effect and skew the study results. Unobtrusive testing helped ensure the provision of consistent and quality assistance to the patron as it aided in the follow-up of the original service. Hernon and McClure (1987b) believed that unobtrusive testing worked if the results affected service guidelines not personnel decisions.

Hernon and McClure (1987a) cited more than 25 unobtrusive studies in their book, Unobtrusive Testing and Library Reference Services, and found accuracy statistics for ready reference questions was about 55\% (Paskoff, 1991, p. 183). They conceived the term "half-right" reference for these statistics (pp. 3-4). Hubbertz (2005) concurred with Hernon and McClure's assessment of low success rates. Saxton and Richardson (2002) suggested that the statistics resulted in analogous numbers since a comparable population took the test each time. Hubbertz suggested that the statistics improved or worsened based on the level of difficulty of the questions. 
If patrons expected a high level of service from reference transactions, the use of unobtrusive testing was one method of assessing the accuracy of the answer and the reference skills of the librarian (Dilevko, 2000). Durrance and Fisher (2003) suggested unobtrusive testing focus on the interviewer not the question to determine successful transactions. Jensen (2004) believed that unobtrusive techniques threatened both patron and librarians' privacy when the researcher failed to eliminate identifying details (p. 143). He proposed that unobtrusive methods have been used to "observe librarian discourse and behavior," "study patron behavior," and "train and monitor the performance of, online librarians under their supervision" (p. 144).

Hubbertz (2005) neither supported nor opposed the use of unobtrusive testing. He recommended unobtrusive testing to adhere to three objectives. The first objective called for the test to be administered consistently. The second objective stressed that the test measured "relative performance" only. The third objective addressed the use of results for evaluating the impact of service on collections and service delivery.

Kellehear (1993) found that unobtrusive studies addressed actual behavior rather than stated behavior. Unobtrusive testing offered opportunities for longitudinal studies and it was relatively safe to the subject, replicable, and inexpensive. The privacy of the subjects was also critical. The only drawback appeared to be maintaining secrecy.

Aluri (1993) found unobtrusive evaluations conducted by outsiders problematic since the evaluators did not work in the environments they studied. They had no personal knowledge of the team that provided the reference service. Improvements to reference service involved all vital team members and relied on ongoing observation. Unobtrusive testing gave a fixed snapshot of service. Aluri suggested that unobtrusive testing worked 
to determine accuracy; however, the behavior of the librarian was more difficult to measure. Another problem identified by Aluri was the trust library administration placed in librarians as it related to unobtrusive testing.

Baker and Lancaster (1991) reviewed unobtrusive studies conducted worldwide that continually cited the accuracy rate of librarians to be at 55\% (p. 246). Elzy, Nourie, Lancaster, and Joseph (1991) conducted an unobtrusive test using 190 questions targeting 19 librarians at the Milner Library at Illinois State University (p. 454). The study evaluated accuracy, the interaction between staff member and student, library conditions at the time of the interaction, and potential improvements to service. The authors stressed the need for answers to be accurate, not just acceptable by the patron. Paskoff (1991) discussed an unobtrusive study that had proxies telephoning six reference questions to 51 academic health sciences and hospital libraries that resulted in 306 responses (p. 182). The questions were designed to reduce the need for reference interviews. The responses showed a $63.4 \%$ accuracy rate plus $25.2 \%$ referrals, $3.6 \%$ inaccurate answers, and $7.8 \%$ no responses (p. 182).

Head and Marcella (1993) supported results of the unobtrusive studies conducted to date, but they decided to conduct their own study to see if they could determine areas for improvement of service. They opted to use biographical questions for their study. Students and proxies could not tell if the library staff person was a librarian. The proxy noted staff approachability, reference interview, suggested sources, and referrals. While the study revealed approachable staff, it indicated lack of reference interviewing, lack of a variety of formats, indifference to the students' knowledge, and about a $60 \%$ response with either partial or complete information provided to the student (p. 13). 
Head, Marcella, and Smith (1995) recommended unobtrusive testing by professionals for accuracy in assessment. The experts understood the difference between an outstanding response and a mediocre response. Roy (1995) confirmed replication of the $55 \%$ rule through unobtrusive studies since the 1960s (p. 218). When librarians remained oblivious to testing, they correctly responded to little more than half of the questions asked. Seay, Seaman, and Cohen (1996) supported the use of unobtrusive testing when performance results helped to improve the service. Unobtrusive testing was "perhaps the best way yet known to study scientifically the reference transaction...used [mainly] to test the accuracy of reference answers" (Tygett, Lawson, \& Weessies, 1996, pp. 270, 272).

Murfin (1995) described unobtrusive testing as invalid since questions asked may not represent questions typically asked by a library's population. The Wisconsin-Ohio Reference Evaluation Program (WOREP) results discussed by Murfin showed how the 9,779 public library transactions conducted in 121 libraries and the 6,925 academic library transactions conducted in 71 libraries accounted for less than $25 \%$ of in-person reference questions; however, many asynchronous library services answered only factual type questions (p. 235).

Dilevko and Dolan (1999) conducted an unobtrusive study of full and selective government document depository libraries in Canada by having paid proxies ask 15 questions to each of 104 libraries for a resulting 488 responses (p. 1). Comprehensive answers occurred $29 \%$ of the time and when partially complete answers were added to the total, the rate increased to $42 \%$ (p. 1). Incorrect or lacking responses occurred $38 \%$ of the time (p. 1). Referrals occurred nearly $20 \%$ of the time (p. 1). 
Ross and Nilsen (2000) reviewed the second phase of a study designed to assess the success rate of reference sessions. Some of the same problems that plagued the first study in the early 1990s plagued the second phase of the study although the success rate increased from $60 \%$ to $69 \%$ (p. 147). Problems identified included poor reference interviewing skills, referrals without facilitation, and failure to follow up with the patron.

Baker and Field (2000) used unobtrusive testing to evaluate services received by students during an on-site visit to an academic library. One of the study results showed how the behavior of reference librarians influenced the student's opinion about services received. The authors reminded librarians that the effortless availability of Internet resources affected students, so they needed to develop exceptional customer relations. Another result was the continuing need for librarians to read current literature about reference interviewing techniques and apply what they learn.

Norlin (2000) discussed secret shoppers who had long been part of the business sector to evaluate company and competitor behavior, prices, and products. They helped identify benchmarks (Cocheo, Harris, \& Kirk, 2003; Czopek, 1998). Tesdell (2000) discussed the mystery shopper approach used by nine public libraries in Michigan to assess the quality of service to patrons. Trained researchers served as proxies and they placed 60 telephone calls to each library's circulation and reference desks. The proxies placed telephone calls throughout the day and three trained panelists scored the responses. Criteria included accuracy, comprehensiveness of responses, referrals to other employees, comprehensiveness of instructions provided, ambiguous information, service minded approach, personal interaction, and added value provided by the staff person. The 
libraries scored 6.4 on a scale of 1 to 7 for accuracy and helpfulness, but the score dropped to 5.4 when value added was considered (p. 145).

McClure, Lankes, Gross, and Choltco-Devlin (2002) supported the use of unobtrusive testing to determine accuracy of the response as the patron anticipated a correct answer to his or her question. They found it less threatening than other types of assessments. The results became tools for the staff to improve the quality of their service. They also recommended the use of unobtrusive testing as an assessment measure for digital reference with the responses evaluated by an objective party with all distinguishing details removed from the responses.

Kaske and Arnold (2002) asked about the ethics of unobtrusive studies and the use of false questions. Unless some balance existed in the asking of the questions, benchmarks could not be established. The identity of the librarian also appeared problematic, but methods for concealing the person's identity existed. Representation of the fictitious patron would actually be the educational level of the student, i.e. graduate student.

The main drawback to unobtrusive testing appeared to be the types of questions used. The questions needed to represent commonly asked questions, but ones of varying difficulty. They could not be questions that frequently recurred. Most questions like this fell into the ready reference category. Daniel (2003) showed that little difference appeared between reference questions asked through written requests and those asked electronically through e-mail. 


\section{Barriers and Issues}

The literature did not adequately address the effectiveness of digital reference for DL students, nor did it address how asynchronous library support services affected the academic experience of DL students. Casey, Sochrin, and Race (2002) believed one of the greatest challenges to DL librarians was the lack of understanding the student had of the need for and the value of DL library services. The provision of asynchronous library support services had a short history with an inadequate body of anecdotal and empirical research (Abels, 1996; Abels \& Liebscher, 1994; Bristow, 1992; Bushallow-Wilbur, DeVinney, \& Whitcomb, 1996; Garnsey \& Powell, 2000; Janes, Carter, \& Memmott, 1999; Lankes, 1998; Roysdon \& Elliott, 1988; Schilling-Eccles \& Harzbecker, 1998; Sloan, 1998b; Still \& Campbell, 1993; Whitaker, 1989). Jones (2002) ascribed some of the blame on academe's efforts to introduce quickly the Internet as a tool to facilitate educational opportunities. Carter and Janes (2000), as well as Peters (2000), attributed the lack of substantial literature to the current evolution of reference service. Schneider (2000) and Janes (2002b) indicated that e-mail reference service gained recognition when librarians received e-mail accounts around the mid-1990s (p. 12). Many libraries initiated e-mail service within the last decade, but numerous models existed with varying eligibility policies for patrons and the types of questions they might ask (Bao, 2003). In many cases, the service appeared buried on library Web pages thereby reducing its usefulness (Bao; Mudrock, 2002; Schneider, 2000).

Research documented decades of reference service studies; however, a set of standards replicable by all libraries appeared difficult to determine (Dewdney \& Ross, 1994; Durrance, 1989; Hernon \& McClure, 1986; Norlin, 2000; Richardson, 2002). 
Saxton and Richardson (2002) suggested the absence of an accepted definition of reference service, independent variables, and outcome variables contributed to inconsistent studies of quality reference service. They also determined that many of the existing studies were flawed due to sampling techniques and sample sizes, statistical procedures, substantiation of existing studies, and use of theory (p. 41).

Lankes, Gross, and McClure (2003) suggested that quality standards indicated an expectation of service; however, such expectations remained unique to each individual library. Standards addressing most situations included courtesy, accuracy, user satisfaction, repeat visitors, awareness of the service, and associated costs (p. 405). Performance measures facilitated the achievement of quality standards. These included measures based on the aforementioned standards, as well as transaction analyses and time on task. Katz's categories for reference queries, as detailed by Mudrock (2002) and the essentials of the digital reference interview as suggested by Kaske and Arnold (2002), might have formed measurable standards. Metrics had yet to be developed for online reference services (Burek-Pierce, 2002; Lynch, 2002). Without measures, benchmarks appeared ill-defined.

Frederiksen (2002), Lessin, McGinnis, and Bean (2002) suggested the need to structure an evaluation tool in response to the need for assessment. Frederiksen proposed the use of the ALA's ACRL Guidelines for Distance Learning Library Services (2004a) to do so. In existence since the late 1960s, the Guidelines provided a source for libraries attempting to establish and evaluate DL library services by offering potential guiding principles, standards, criteria, outcomes, and assessment tools (p. 261). 
The unobtrusive study conducted by Hernon and McClure (1986) created some debate regarding the methodology employed. Lynch (2002) stressed the 55\% rule resulting from the Hernon and McClure study was accepted as the norm. Burton (1990) also found the $55 \%$ rule demonstrated in a number of subsequent reference studies. Hernon (1996) admitted the $55 \%$ rule was not the only answer to excellence in service, but was one of the measures. "Effectiveness, efficiency, value, service quality, [and] satisfaction" were contributory (p. 171).

Students realized the Internet did not always provide them with the information they seek, but they failed to seek additional reference assistance from librarians in their search for quality information (O'Sullivan \& Scott, 2000). They believed the Web fell short of providing credible resources, yet they had difficulty asking experts for help (Agre, 1994; OCLC, 2002; Valentine, 1999). The Pew study of Search Engine Users found that "most searchers are naïve about search engines and search results" and it indicated that only “one in six Internet searchers can consistently distinguish between paid and unpaid search results" (Fallows, 2005, pp. iv, 18). Without guidance, the student was less likely to know the expectations of college level research and the concern existed about the Internet enabling the development of inadequate research skills and habits (Jones, 2002). Jones' study showed nearly $73 \%$ of students $(n=2,054)$ surveyed utilized Internet resources in lieu of library databases, Web sites, and catalogs (p. 12).

Research indicated students showed discomfort about asking for assistance but they found their own research skills inadequate (Kuhlthau, 1988; Massey-Burzio, 1998; Mellon, 1986). Their trepidation about asking for assistance led them to friends, family, and classmates before asking a librarian for assistance (Valentine, 1999). The accuracy of 
information remained critical to them; yet, they often failed to discern quality online resources from inferior products (Jones, 2002). They also feared appearing ignorant in front of a library professional (Mellon, 1986).

This study relied on the opinion of DL students at Indian River Community College, DL librarians at Florida's 28 community college libraries, the researcher, and the assessed responses received from e-mail reference inquiries.

\section{Summary of the Known and the Unknown}

The literature review supported the impact of distance learning on higher education and the need to provide equitable library services to students in the digital environment. The obvious absence of library services from support services necessary for DL students emerged as a concern. Although academic libraries began developing digital services for students, the lack of standards and measurements of digital services was readily apparent. Due to the student's reliance on unsubstantiated Internet sources for reference and research needs, digital reference services appeared to be one of the more significant services proffered by academic libraries. The study focused on asynchronous reference service since it had a longer history than synchronous reference service and literature dealing with mostly anecdotal information.

Since nationally or regionally accepted standards and measures for digital reference service could not be identified through the literature, the researcher developed an independent set of instruments for measurement based on criteria useful in the digital environment and criteria useful in general reference services. Studies conducted of traditional and telephone reference showed that approximately $55 \%$ of the responses were 
accurate. Without documented research in the digital reference services area, the $55 \%$ rule might or might not have applied to asynchronous reference responses. One instrument addressed the measurement of responses made to students in the digital environment. One instrument addressed the policies and procedures in place at the libraries being studied, as well as the opinions of DRSPs offering asynchronous digital reference service.

\section{Contributions to the Field}

The digital reference environment offered opportunities for contribution to existing research in the field of reference services and communication between the librarian and the student. One of the primary benefits was realizing whether the results of this study would reflect results from three decades of reference studies. The second primary benefit was to determine whether the communication techniques employed by librarians in the digital environment succeeded with the student in the online environment. The study might have contributed to the use of measurement instruments within the digital reference environment. The results might also pinpoint areas needed to educate future librarians or to re-educate practicing librarians. The study might support the need for nationally or regionally accepted standards or models for the provision of digital reference services. In order to garner contributions to the field, this study examined the adequacy of asynchronous e-mail reference services offered to DL students at Florida's 28 community college libraries and the contribution of library staff to the students' online learning community. 


\section{Chapter 3}

\section{Research Methodology}

\section{Research Methods Employed}

The purpose of this study was to examine the adequacy of asynchronous e-mail reference services offered to DL students at Florida's 28 community college libraries and the contribution of library staff to the students' online learning community. Existing literature did not adequately address the effectiveness of digital reference for DL students, nor did it address how asynchronous library support services affected the academic experience of DL students.

This study was accomplished using an interview instrument, unobtrusive testing techniques, and an analysis of the unobtrusive testing results. DRSPs, selected by their respective administrators, were interviewed to determine the digital reference policies and procedures in place at their respective institutions and to learn more about their personal opinions regarding the provision of digital reference. Reference questions were asked unobtrusively of each library's digital reference service. These techniques were used to assess the effectiveness of asynchronous reference service, particularly the accuracy of the responses to reference inquiries and the manner by which the DRSPs treated patrons. The researcher conducted the analysis by comparing the researcher's and the students' assessments of the unobtrusive test results. Based on results of the interviews and the unobtrusive portion of the study, the researcher determined whether 
asynchronous library support services within Florida's community college system adequately addressed reference requests made digitally by students. A work flow diagram of the methodology used for this study has been included (see Appendix A). Interviews, the unobtrusive study, and the researcher/student assessments occurred after the researcher received IRB approval from Nova Southeastern University (see Appendix B).

The researcher asked these questions. Do asynchronous library support services adequately address reference requests made digitally by students? Do librarians respond to students in a timely manner? Do they ask additional or follow-up questions to comprehend fully the student's research request? How do librarians respond to student inquiries? Do they provide students with accurate information? Do they cite the sources used to answer reference questions?

Do these services enhance the student's online learning community? Does the librarian initiate an open and inviting dialogue? Does the librarian attempt to personalize the experience for the student? Does the librarian invite the student to use the service again should additional questions arise?

The researcher addressed the research questions dealing with the timeliness of the response, follow-up questions for the student, DRSP attitudes, accuracy of the response, invitations to utilize the service again, and sources used during the unobtrusive portion of the study. The unobtrusive portion of the study was conducted prior to the interviews to reduce the possibility of disclosing the nature of the study. Research indicated that an unobtrusive study was one way to test subjects in a natural setting without causing them to alter their usual professional habits in an effort to study the reference transaction (Crowley \& Childers, 1971; Cullen, 2002; Jirjees, 1981; Kaske \& Arnold, 2002; Tygett, 
Lawson, \& Weessies, 1996). Crowley and Childers believed that this technique “increases the validity of the data" (p. 25). Furthermore, Whitlatch (2001) supported its use for digital reference.

The second set of research questions dealing with the DRSP's dialogue and the DRSP's attempted to personalize the experience for the student were addressed through the unobtrusive portion of the study and the DRSP interviews. As well as identifying whether or not the action took place, the researcher sought clarification through the interviews to ascertain whether the DRSPs believed they had a role in the student's online learning community and whether they acknowledged the need to communicate with the student in an open and responsive manner.

\section{Specific Procedures Employed}

\section{Selection of the Population}

Florida's 28 community colleges supported the educational needs of students, employees, and community members in their respective service districts. The Florida Department of Education divided the community colleges into small, medium, and large sized institutions according to full-time enrollment figures. Since each group had either 9 or 10 institutions, the researcher opted to employ the State's size breakdown. The population was then pre-existing. These 28 community colleges had shared a library management system since the early 1990s. Due to the shared nature of their relationship with the library management system, the community college libraries also explored other opportunities for sharing services and resources during the past decade. Services and 
resources included document delivery, information literacy initiatives, joint leases of online resources, database and Internet skills training, and reciprocal borrowing. The libraries were accustomed to conducting surveys and training as a group despite differences in their respective missions. The data was analyzed in the three group sizes and for the state, as a whole.

The researcher opted to interview a DRSP from each of the 28 community colleges; however, the respective library administrators made the choice as to which DRSP was interviewed. The selection of participants was random, as the researcher could not predetermine the DRSP that each of the administrators selected.

The unobtrusive portion of the study dealt with the DRSPs who handled digital reference services at their respective institutions. The population of library staff assigned to their institution's digital reference services could not be ascertained prior to the start of the study for fear of disclosing the unobtrusiveness of the study. Staffing varied from library to library, so the number of employees assigned to particular library functions was difficult to quantify without contacting individual libraries. In the case of digital reference service, both librarians and support staff might have been involved. Some libraries might have had a triage system in place, others might have had one person assigned to the service, and yet others might have rotated the service amongst its staff. The minimum number of participants was expected to be at least 28 DRSPs, one per institution. In essence, the selection of participants was random as the researcher could not pre-determine or recruit participants.

The researcher selected student assessors from three sources. The first source was a pool of students who had already taken or who were currently enrolled in DL classes. The 
second source was a pool of students who had already taken or who were currently enrolled in blended classes (classes with reduced seat time combined with online sessions). The third source was a pool of students who had asked for help from a librarian using e-mail or an online form. These pools helped ensure that the student assessors would have some familiarity with the online environment. These students constituted the group, which assessed the responses generated from the unobtrusive portion of the study. A maximum of 84 students were needed if each one of the community college libraries answered all 15 reference questions. Each student assessed five separate reference responses. Using a variety of students enabled a more diverse set of assessments. Student volunteers were random, so the researcher could not have pre-determined which students might respond to the request for assistance.

The researcher sought permission from the administration at IRCC to involve both the institution and its students in the study. The Vice President for Academic Affairs approved use of the institution and its students for the study. A letter of approval was requested and received for inclusion in the IRB documentation (see Appendix C).

\section{Reference Questions}

The reference queries used in this study reflected questions characteristic of Florida's community college students. The answers to the inquiries were answerable using online resources and standard traditional sources (i.e., MLA Handbook and APA Publication Manual) since the researcher had no immediate access to the collections at all of Florida's community college libraries. While the answers to the queries did not necessarily have to come from a particular source, the response had to be accurate and the 
resource had to be current. To that end, the researcher reviewed the literature to determine the types of questions typically asked of digital reference services and examined the questions submitted to IRCC's “Ask a Librarian" digital reference service. The list of reference questions comprised queries asked of the researcher's "Ask a Librarian" service during the past two years, as well as adaptations of other types of reference questions. Jirjees (1981) said the use of actual questions provided legitimacy despite the use of unobtrusive data gathering.

A list of 20 reference questions was developed for electronic submission to the digital reference services at Florida's community college libraries. Five questions were culled from the list leaving 15 questions for the study. The questions were written to reduce the need for a reference interview although a background story was written for DRSPs who engaged in a more thorough reference interview. Although many of the questions were previously asked or adapted, the researcher explored and answered each of the questions before using them in the study. Crowley and Childers (1971), as well as Whitlatch (2000), supported the design of questions that were generalizable. The questions were answerable with standard reference sources or with Web based resources.

The types of questions included one biographical question, two citation questions, two known item questions, two medical questions, two statistical questions, one technical question, one ready reference question, one current event question, one business question, and two reference questions related to course research assignments. Garnsey and Powell (2000) used ready reference, research, genealogy, technology, document delivery, bibliographic verification, and other as reference question categories (p. 250). Of the 15 final questions, 33.3\% were based on questions adapted from other reference questions 
asked of the researcher or from other information retrieval experts, and $66.7 \%$ were based on questions received electronically by the researcher. Crowley (1985) believed that tests could not consist solely of easy questions or the accuracy rate will be high.

The biographical question was based on an IRCC English I course obituary writing assignment. IRCC English II students asked the APA and MLA citation questions. An IRCC student and an IRCC faculty member asked the known item questions. One of the statistical questions was adapted from a question asked by Dr. Steven Zink during one of his NSU information science classes. A student in an IRCC speech class asked the second statistical question. The current event question stemmed from a request by the Florida Legislature to initiate a statewide beach warning flag system. One of the medical questions was adapted from an IRCC patron's question regarding medications for a leg injury. The second medical question was adapted from an unobtrusive study and modified by a member of the IRCC nursing faculty (Paskoff, 1991). The ready reference question was based on the researcher's hometown. The researcher fielded both of the research questions and the business question. The researcher received the technical question several times per week.

\section{List of Reference Questions}

The final list of 15 questions included the question, (a) a background story should the DRSP ask additional questions, (b) the name of the proxy, (c) the e-mail address of the proxy, (d) the answer, and (e) recommended resources. Elzy, Nourie, Lancaster, and Joseph (1991) stressed the need for a "cover story" to protect the secrecy of the study (p. 457). Basic responses should have also included some of the following instructions: how 
to access LINCC for books, how to determine suggested search terms, how to conduct a search in LINCC, how to retrieve the books found in LINCC, how to obtain a barcode, how to access the databases, how to login to the databases, how to set up a search strategy, how to conduct a search in the databases, how to conduct a Web search, how to determine a usable result, how to cite traditional and online sources, or how to arrange for reciprocal borrowing or an interlibrary loan. Contact information for specific questions might have also been shared with the proxy.

\section{Questions}

1. Hi, I have to write a short paper on somebody famous. I want to do it on the female athlete who died last year. What's her name? What did she play again? Thx...

a. Background: I am actually writing an obituary for one of my assignments. I remember my teacher saying that the athlete was a black lady.

b. Lou

c. Jerseygir1731@rock.com

d. Althea Gibson was the name of one female athlete who died September 28, 2003. Known primarily for playing professional tennis, Althea Gibson also played professional golf. Before she entered professional sports, she played basketball, paddle tennis, and tennis.

e. Recommended sources included newspapers, magazines, books, or biographical sources. The intent was to have the DRSP identify the name of the athlete and to recommend authoritative biographical resources as evidence. 
2. Can you help me set up a reference using APA? The article is called Predicting Serial Killers' Home Base and David Kanter wrote it. I don't know how to set it up. Thank you very much.

a. Background: I found this article in one of the library's databases. I want to add it to my works cited page, but I don't know how to set up the APA stuff.

b. Chris

c. Golfpro3550@yahoo.com

d. The DRSP might have provided two different responses based on the utilization of an online database.

If the DRSP addressed the question without knowing if the article was retrieved from an online database:

Canter, D., Coffey, T., Huntley, M., \& Missen, C. (2000). Predicting serial killers' home base using a decision support system. Journal of Quantitative Criminology, 16, 457-478.

If the DRSP learned that the student used an online database:

Canter, D., Coffey, T., Huntley, M., \& Missen, C. (2000). Predicting serial killers' home base using a decision support system. Journal of Quantitative Criminology, 16, 457-478. Retrieved date, from Academic Search Premier database.

e. The correct response was the American Psychological Association. (2001). Publication Manual of the American Psychological Association (5 $5^{\text {th }}$ edition). Washington: Author. 
3. Does the library have a copy of Lucky Jim on video? I really need to watch it. Keep me posted. Thanks.

a. Background: I am reading the book for class. My group is going to act out a few scenes from the book, so I really wanted to see the videotape.

b. Sandy

c. Bud0315@hotmail.com

d. Lucky Jim is a 91-minute videotape. A search on LINCC, the Florida community college library system, showed that none of the 28 community college libraries owned the videotape.

e. Recommended sources included LINCC, WebLUIS - the online public access catalog for the State University System of Florida, local libraries, OCLC, and a videotape/book source, like Amazon. ${ }^{10}$

4. Where can I buy a copy of Dante's The Inferno translated by John Ciardi. How much will it cost in hardcover? It's a birthday present. Many thanks.

a. Background: I would like to give my doctor a birthday gift. She was very helpful when I was ill this semester. From what I see on her bookcase in her office, The Inferno title would be a perfect gift. I really don't care how much the book costs.

[Note: This question was eliminated from the study due to tremendously slow Web response on the day it was asked. MyDoom worm slowed online access and rendered many outgoing asynchronous requests undeliverable. The slow response time compromised the data collection period].

\footnotetext{
${ }^{10} \mathrm{http}: / /$ www.amazon.com.
} 
b. Pat

c. travelparis@excite.com

d. The title was actually part of Dante's Divine Comedy. The Divine Comedy includes The Inferno, Purgatorio, and Paradiso. The edition translated by Ciardi was more difficult to find as a separate purchase. It was available during the data collection portion of the study through Amazon $^{11}$ for $\$ 53$ and through AlBooks $^{12}$ for $\$ 44.16$. The ISBN is 0393044726 . The locations were found through searches on both AddAll $^{13}$ and FetchBook. ${ }^{14}$

e. Recommended sources included online bookstores, especially those with comparative bookstore information. The researcher had no preference for specific online booksellers. The aforementioned sites were examples based on the researcher's investigation.

5. What was the inflation rate in 1980 for tobacco products? What's the current rate? I need them for an economics class project. Appreciate your time.

a. Background: I am part of a group in my economics class. My group is looking at the changes in the tobacco industry during the last 20-25 years.

b. Hank

c. Gardengir1723@collegeclub.com

d. The answer was 72.0 for 1980 and 473.3 (April 2004) or 473.5 (May 2004) or 476.0 (June 2004).

\footnotetext{
${ }^{11} \mathrm{http}: / /$ www.amazon.com

${ }^{12} \mathrm{http}: / /$ www.albooks.com

${ }^{13} \mathrm{http}: / / w w w . a d d a l l . c o m$

${ }^{14} \mathrm{http}: / /$ www.fetchbook.com
} 
e. The U.S. Census Bureau (2003) defined inflation as "a time of generally rising prices for goods and factors of production. The Bureau of Labor Statistics samples prices of items in a representative market and publishes the result as the CPI" (p. 471). The best source for inflation figures for this question was the federal government. For the 1980 data, the source was the Statistical Abstract of the United States ${ }^{15}$ or the Bureau of Labor Statistics (BLS) Web site. ${ }^{16}$ For current or historical data, the source was the U.S. Department of Labor's BLS Web page ${ }^{17}$ or the CPI Detailed Report journal. An e-mail was sent to cpi_info@bls.gov for confirmation. Gibson, an economist with the CPI, confirmed the aforementioned answer (S. Gibson, personal communication, October 25, 2004).

6. Hi! What do the colors on the lifeguard flags mean?

a. Background: I am a new resident in Florida. I want to be able to tell when I drive by the beach whether or not I can go into the water.

b. Jamie

c. zoidsbuilder@1ycos.com

d. Colors

i. Green: Low hazard, calm conditions

ii. Yellow: Medium hazard, moderate surf and/or currents

iii. Red: High hazard, rough conditions, strong surf and/or currents iv. Red over Red: Water closed to the public

${ }^{15} \mathrm{http}: / / \mathrm{www} . c e n s u s . g o v /$ statab/www

$16 \mathrm{http}: / /$ stats.bls.gov

${ }^{17} \mathrm{http}: / /$ www.bls.gov/cpi 
v. Purple: Marine pests present

At the request of the Florida Legislature, the Florida Department of Environmental Protection coordinated the development of a uniform beach warning flag system for Florida. The Florida Department of Environmental Protection worked with the Florida Beach Patrol Chiefs Association, the United States Lifesaving Association, and the International Lifesaving association to develop a uniform warning flag program for use by Florida's beachfront and coastal communities. Florida was the first state in the nation to standardize and institute a statewide beach flag system. Florida's Governor Bush signed House Bill 1395 (2005), which allowed the posting of warning flags on unguarded beaches.

e. Recommended sources included the Florida government, particularly the Fla. Stat. $§ 380.276$ (2004), the Florida Department of Environmental Protection, ${ }^{18}$ and articles from newspapers or magazines (for example, First Coast News, ${ }^{19}$ News Herald, ${ }^{20}$ Beach Warning Flags, ${ }^{21}$ and New Flags Flying. ${ }^{22}$

7. Where can I find patient warnings for Celebrex? It's an anticonvulsant.

a. Background: I found the medicine in the cabinet and wanted to check for information about taking it with milk.

b. Casey

c. virtualmom@myway.com

${ }^{18} \mathrm{http}: / /$ www.dep.state.fl.us/cmp/programs/flags.htm

${ }^{19} \mathrm{http}: / / \mathrm{www}$.firstcoastnews.com/ news/news-article.aspx? storyid=18737

${ }^{20} \mathrm{http}: / /$ www.newsherald.com/articles/2002/2003/18/1o031802a.htm

${ }^{21} \mathrm{http}: / / \mathrm{www} . \mathrm{msnbc} . \mathrm{com} / \mathrm{id} / 5087324$

${ }^{22}$ http://www.wjhg.com/news/headlines/572327.html 
d. A confirmation question was required to determine the name of the drug or the medical purpose of the drug that the student seeks. Celebrex ${ }^{\circledR}$ was not an anticonvulsant. It was an anti-inflammatory. Cerebyx ${ }^{\circledR}$ was an anticonvulsant.

e. Recommended sources included Gale's Health and Wellness database or some of the following Web or medical resources: Celebrex, ${ }^{23}$ Cerebyx, ${ }^{24}$ U.S. Food and Drug Administration, ${ }^{25}$ MayoClinic, ${ }^{26}$ MayoHealth,${ }^{27}$ Medline,${ }^{28}$ National Institutes of Health (NIH), ${ }^{29}$ National Library of Medicine (NLM), ${ }^{30}$ PDR (Physicians' Desk Reference), RxList ${ }^{31}$ or other reputable Web sites. The sources did not substitute for the expertise of medical professionals and all literature should have been read carefully to determine the name of the product.

8. I'm writing an argumentative essay how do I find the material I need for my thesis?

The topic is banning smoking in public places. I probably need 3 articles.

a. Background: This is the first time I am writing an essay for class. I don't know how to get started. The essay is 1250 words.

b. Loren

c. Leosun31@nescape.net

${ }^{23} \mathrm{http}: / / \mathrm{www} . c e l e b r e x . c o m$

${ }^{24} \mathrm{http}: / / \mathrm{www}$.cerebyx.com

${ }^{25} \mathrm{http}: / /$ www.fda.gov

${ }^{26} \mathrm{http}: / /$ mayoclinic.org

${ }^{27} \mathrm{http}$ ://www.mayohealth.org

${ }^{28} \mathrm{http}: / /$ medlineplus.gov

${ }^{29} \mathrm{http}: / / \mathrm{www} \cdot$ nih.gov

${ }^{30} \mathrm{http}: / /$ www.nlm.nih.gov

${ }^{31} \mathrm{http}: / / \mathrm{www} . r x$ list.com 
d. The DRSP needed to learn more about the assignment and the resources the student had already consulted. If the DRSP opted to bypass this step, the recommended sources were optimum.

e. Recommended sources included a few databases, like Academic Search Premier, CQ Researcher, Current Controversies, Facts on File, Issues and Controversies, OmniFile Full Text Mega, Opposing Viewpoints, or SIRS Student Resource Center, or credible Web sites. More general databases, like OmniFile Full Text Mega, required an effective search strategy to garner the best results, so suggested strategies should have accompanied the response.

9. Hi there. I'm getting married in a few months. My boyfriend gave me a list of his relatives. I can't find the zip for Menlo Park Ter in NJ. He said it's near the Edison tower. Hope you can find it for me. Thanks for your help!

a. Background: I cannot find the zip code myself, so I thought I would ask for help. These relatives would get upset if they weren't invited, so I don't want the mail to get lost. The address is 83 Isabelle Street.

b. Sam

c. Racefan11358@go.com

d. The ZIP code was 08840. Menlo Park Terrace was located in Woodbridge Township, Middlesex County, New Jersey. It was approximately 1.8 miles from the Edison Tower.

e. The ZIP code was not accessible via the U.S. Postal Service's database, so the DRSP needed to do a bit of research to assist the student. If the DRSP read closely, the city name was Menlo Park Terrace, not Menlo Park. These cities 
were found in two different locations in New Jersey, but they were located adjacent to one another. Menlo Park Terrace was part of Woodbridge Township and the Menlo Park Terrace mail was routed through Metuchen. Recommended sources included a commercial atlas, gazetteer, Mapquest, a New Jersey public library, the local post office, or the New Jersey Department of Transportation's county maps.

10. Who can help me with a problem I am having with searching the library databases online. I log in fine, get all the way through to Connect to Database, and get "Authentication failed due to insufficient credentials." My card and PIN work fine on the library computers. Please help or direct me to who can assist me. Thanks.

a. Background: I started my research while in the library, but when I got home to continue it, I ran into problems with logging in.

b. Katie

c. shortaypunker@aol.com

d. The student indicated success using the library barcode and PIN at the library itself, so other possible technological problems caused failure.

e. Recommended solutions included minimizing AOL and maximizing Internet Explorer, updating the browser, switching to Internet Explorer from other browsers, disabling a firewall for the duration of the search, or lowering the security setting. Specific contact information was also recommended.

11. Hello, I need to find some data on highway violence in Florida. Where can I go? Thanks so much. 
a. Background: I am doing a paper on road rage, so I want to add some statistics. My teacher said that she really likes to see students go the extra mile.

b. Miller

c. Trask177@excite.com

d. Florida statistics could have been found on Florida State's Web site, specifically, the Florida Highway Patrol ${ }^{32}$ and the Florida Department of Highway Safety and Motor Vehicles. ${ }^{33}$

e. Recommended resources included Florida Highway Patrol ${ }^{34}$ Web site or resources, Drug Interdiction, Forfeiture, and Highway Violence, Florida statistical sources listed on the Florida Electronic Federal Depository Library ${ }^{35}$ Web site, or the Florida Department of Highway Safety and Motor Vehicles ${ }^{36}$ Web site or resources. Online databases, such as Academic Search Premier, Custom Newspapers or Florida newspaper databases, InfoTrac, OmniFile Mega, or Student Resource Center provided some articles with data. The online catalog showed Florida statistical titles or documents. A browser search resulted in the data sought by the proxy (highway-violence Florida data OR statistics).

12. I am having a hard time finding some books on my topic. I checked LINCC and I am sure I don't know what I am doing, can you help? I want to find some books about

$32 \mathrm{http}: / /$ www.fhp.state.fl.us

${ }^{33} \mathrm{http}: / /$ www.hsmv.state.fl.us

${ }^{34} \mathrm{http}: / / w w w . f h p . s t a t e . f l . u s$

${ }^{35} \mathrm{http}: / /$ www.uflib.ufl.edu/fefdl

${ }^{36} \mathrm{http}: / /$ www.hsmv.state.fl.us 
self-image and how it starts with Barbie. Does the library have books like this on the shelf? What do I do if you don't have any books? I need at least two books for my bibliography. Thanks.

a. Background: I have to write a 2000 word paper. I remember some news stories about Barbie's actual dimensions.

b. Hope

c. Westwingfan1985@yahoo.com

d. Two books emerged specifically mentioning Barbie in the title; however, a search of women and (body image or self-esteem) may have resulted in related titles.

i. Body Burden, Living in the Shadow of Barbie by Stacey Handler

ii. Adios, Barbie: Young Women Write about Body Image and Identity by Ophira Edut (editor)

e. The recommended resource was LINCC to locate Barbie book titles within the Florida community college library system. Other resources included NetLibrary, Books in Print, Amazon, ${ }^{37}$ WebLUIS - the State University Library System of Florida, or the Web for several definitive sites to find titles that might not have been in the LINCC database. If the books were not available at the student's library, the local public library, local university library, or through interlibrary loan were recommended as resources.

${ }^{37} \mathrm{http}$ ://www.amazon.com 
13. Help. I am writing an MLA paper. I need to set up a reference. It looks really weird. Okay, the book has a bunch of chapters written by different people. So far, I have this-

Editor: William Schutte

Title: Twentieth century interpretations of a portrait of the artist as a young man Publisher: Prentice Hall

Date: 1968

Author of the chapter: Lee T. Lemon

Chapter name: A portrait of the artist as a young man: motif as motivation and structure. It starts on page 41 and ends on page 52. This is where it gets weird. Way at the bottom of page 41, it has different info. I'm gonna type it just like the book says. "A Portrait of the Artist as a Young Man: Motif as Motivation and Structure" by Lee T. Lemon. From Modern Fiction Studies, XII (Winter 19661967), 439-450. Copyright 1965 by Purdue Research Foundation, Lafayette, Indiana.

a. Background: I found this article in a book I was using. I want to add it to my bibliography, but I don't know how to set up MLA.

b. Mike

c. Mike23175@netscape.net

d. From Gibaldi, J. (2003). MLA Handbook for Writers of Research Papers (6th edition). New York: Modern Language Association, the DRSP should have referred the student to section 5.6.7 for a reprinted article in an anthology Citing previously published work. The reference provider might also have 
double-checked the city of publication. A check in LINCC could have answered that question.

e. Recommended resources included the aforementioned edition of the $M L A$

Handbook, the student's instructor, credible Web sites that provided accurate references to the MLA Handbook, $6^{\text {th }}$ edition, examples based on the $6^{\text {th }}$ edition, or referrals to the source as an anthology.

Lemon, Lee T. "A Portrait of the Artist as a Young Man: Motif as Motivation and Structure." Modern Fiction Studies 12 (1966-67): 439-50. Rpt. in Twentieth Century Interpretations of A Portrait of the Artist as a Young Man. Ed. William Schutte. Englewood Cliffs, NJ: Prentice Hall, 1968. 41-52.

Or

Lemon, Lee T. "A Portrait of the Artist as a Young Man: Motif as Motivation and Structure." Modern Fiction Studies 12 (1966-67): 439-50. Rpt. in Twentieth Century Interpretations of "A Portrait of the Artist as a Young Man." Ed. William Schutte. Englewood Cliffs, NJ: Prentice Hall, 1968. 41-52.

14. Hi, I have to do a speech in class on the "Hershey" company. I need to know the date of incorporation, the main lines of business, last year's sales, profits, and federal income tax paid. Where can I go?

a. Background: I have the annual report, but it doesn't include all of these details.

b. Tom

c. Tomahawk1985fl@hotmail.com 
d. The student could have used a search engine to conduct a search for the Hershey Company. The result was the company's current Web page. ${ }^{38}$ Tabs across the top of the page read: About Hershey Foods, Investor Relations, Newsroom, and Career Opportunities. The student should click on the tab labeled Investor Relations. Under this tab, the student could have found a number of sub-topics that answered the aforementioned questions.

e. Recommended sources included the corporate ${ }^{39}$ Web site and business report databases. The corporate Web site was the best place to start for current and accurate information. The student might have checked online business databases or Web sites, such as Business Source Elite, Hoover's Online Business, and Lexis Nexis. Other online sites included BigCharts ${ }^{40}$ or Yahoo biz. ${ }^{41}$

15. Is cellulitis hereditary?

a. Background: I was diagnosed with cellulitis, and I wanted to know if my sister will also get it?

b. Joseph

c. Shadowone733@netscape.net

d. The DRSP might have confused cellulitis with cellulite, so the DRSP might have double-checked the condition. The DRSP might have rendered a judgment rather than suggesting that the student seek a medical opinion from

\footnotetext{
${ }^{38}$ http://www.hersheys.com

${ }^{39} \mathrm{http}: / / \mathrm{www} \cdot$ hersheys.com

${ }^{40} \mathrm{http}: / /$ www.bigcharts.com

${ }^{41} \mathrm{http}: / /$ www.finance.yahoo.com
} 
a credentialed expert. The DRSP was not a medical expert and could not make medical judgments.

e. The DRSP might have directed the student to his or her medical caregiver for a final expert medical opinion. The DRSP might have recommended some reputable resources for the student to review. The DRSP should have directed the student to reputable medical databases, resources, or Web sites for the student's personal review of the information about the condition with the admonition about making medical judgments. Recommended sources included the National Institutes of Health, ${ }^{42}$ the National Library of Medicine, the MayoClinic ${ }^{43}$ Web site, MedlinePlus,${ }^{44}$ Merck, ${ }^{45}$ medical dictionary, medical encyclopedia, or health and medical databases, such as the Health and Wellness Resource Center. The DRSP could have referred the student to highly credible medical Web sites or reference sources.

\section{List of Interview Questions}

The interview questions addressed digital reference policies and procedures at each of the 28 community college libraries. The researcher also asked the DRSPs about their opinions of asynchronous reference service to gain an understanding of their personal attitudes about asynchronous reference service. The DRSPs provided the service to the students, but the researcher wanted to learn whether the DRSPs believed the service was valuable to students and if they believed that, the service could replicate face-to-face

${ }^{42}$ http://www.nih.gov

${ }^{43} \mathrm{http}: / /$ www.mayohealth.org

${ }^{44} \mathrm{http}: / /$ medlineplus.gov

${ }^{45} \mathrm{http}$ ://www.merck.com/mmhe/index.html 
reference service. Anticipated responses had not been included, as with the reference questions, because the researcher had no preconceived expectations for the DRSPs responses. Whitlatch (2001) maintained that interviews provide a method for extracting the personal observations of interviewees. Novotny (2001) found that personal interviews enabled the participants to communicate their own opinions without losing their individuality in a focus group session.

The library administrators at the 28 community college libraries were asked to approve the interview and suggest the name of a DRSP to be interviewed (see Appendix D). The request discussed the focus of the study, the need to interview DRSPs to gain a better understanding of digital reference services offered locally, the steps taken to maintain the confidentiality of the institution's and the DRSP's identities, and a brief review of the significance to the field of information science. The interviews were conducted in an asynchronous manner in an effort to both provide the DRSPs time to prepare their responses and to maintain the nature of the study of asynchronous services. With the library administrator's approval, each DRSP signed a letter of informed consent prior to the interview (see Appendix E). The letter of consent was similar to the one shared with their respective library administrators. The researcher asked these questions.

1. Does your library have written policies and procedures for AskA e-mail reference service? If so, may I have a copy of them? Please send them to Pat Profeta, 3550 $3^{\text {rd }}$ Place SW, Vero Beach, FL 32968.

2. How many people handle AskA e-mail reference questions at your library? What types of degrees do they each have? How long have they been working in libraries? Their names are not needed. 
3. How do you train your AskA e-mail reference staff?

4. What types of questions does your AskA e-mail reference service typically receive?

5. How does your AskA e-mail reference staff handle questions excluded from your library's policy?

6. What types of questions do you find most appropriate for e-mail reference service?

7. How do you assist patrons who are not included in your AskA e-mail reference policy?

8. How do you develop an online relationship with students who ask for help via email?

9. What do you perceive is your role in developing the students' online learning environment?

10. How do you learn about the student's computer skills, Internet, or research skills?

11. How do you believe the use of e-mail for reference service changed the way library staff conduct the reference interview?

12. Do you survey your e-patrons to assess their satisfaction with the service?

13. How does your library or college monitor your AskA e-mail service for quality?

14. What other methods does your library staff use to judge the effectiveness of its service?

15. How should distance learning students receive reference assistance if library policy restricts the types of questions answered by AskA e-mail reference service? 


\section{Assessment Guidelines}

The researcher designed the Checklist for Quality E-mail Reference Responses guidelines and Scoring Quality E-mail Reference Responses guidelines for analysis of the reference responses (see Appendices F \& G). The researcher and a group of students assessed the reference responses sent to the proxy using the Checklist for Quality E-mail Reference Responses guidelines. The guidelines addressed communication, timing of the response, accuracy of the response, the assessor's willingness to return to the DRSP for assistance, and a comments field. The Scoring guidelines, used for the researcher's analysis, assigned point values and assessment scores for the responses. Although other coding guidelines were reviewed, none targeted all of the areas that the researcher wanted to study (Bristow \& Buechley, 1995; Bushallow-Wilbur, DeVinney, \& Whitcomb, 1996; Daniel, 2003; Duff \& Johnson, 2001; Grogan, 1992; Schilling-Eccles \& Harzbecker, 1998).

\section{Expert Panel}

Prior to conducting the study, the researcher submitted the reference questions, the interview questions, and the coding guidelines to a panel of six library and information science experts (see Appendix H). None of the panel members were Florida librarians and all agreed to preserve the confidentiality of the study. Limitations of the expert panel included the subjectivity of individual judgment, their individual experiences working with reference questions, their interpretation of the question from a professional perspective rather than a student's perspective, their understanding and knowledge of reference resources, the currency of their reference experience, their expertise in a 
particular library department, their expertise in particular library types, and their personal philosophy on unobtrusive methodology.

\section{IRB Approval}

The researcher sought IRB approval from Nova Southeastern University. IRB submission forms and research protocol were submitted to Nova Southeastern University's Office of Grants and Contracts through Dr. James Cannady on May 4, 2004. The researcher received IRB approval from Nova Southeastern University (J. Cannady, personal communication, May 15, 2004) (see Appendix B).

\section{Random Numbers}

To protect the identity of the 28 community colleges and their respective DRSPs, the researcher used random numbers to conceal their identities. The Random 3-Digit Code Number Generator, offered at no cost through the Registrar's Office at the University of Oregon (n.d.), was used. Several tests conducted by the researcher showed the Generator provided unique and non-repeating numbers. Random numbers were assigned to each of the 28 community colleges and their DRSPs. These random numbers were used on the reference responses so student assessors could not identify the institution or the DRSP. The researcher also used them to classify the interview responses and the researcher's assessments of the reference responses by small, medium, and large institutions. 
Proxy

In order to maintain anonymity, the researcher created 15 false names for the proxy setup. A Web search conducted for free e-mail services resulted in a variety of e-mail accounts. Fifteen e-mail accounts were established through various services and each account represented a different proxy's name. The e-mail accounts were generated for receipt of the reference responses. Only the first name of the student was used. If a library Web site posted a digital reference request form and it asked for a last name, only an initial was entered onto the form. Each proxy was identified as a student to be considered a primary client. Childers determined that the proxy should look as if he or she has a legitimate reason to use the service (Crowley \& Childers, 1971).

\section{Collecting Data}

The data collection of the reference questions was done using unobtrusive testing. Used for the past three decades, unobtrusive testing helped to evaluate the adequacy of reference service, experiences with library staff, and patrons' attitudes toward repeat visits (Baker \& Field, 2000; Hernon \& McClure, 1986, 1987a). This method allowed for the optimum response without triggering the Hawthorne Effect. The Hawthorne Effect indicated that an individual's behavior changes when he or she knows that someone is studying it (Tygett, Lawson, \& Weessies, 1996). Such testing helped ensure that the provision of consistent and quality service to the patron did not occur only through obtrusive observation. The researcher remained concerned about discovery and closely analyzed the responses for discovery. Libraries discovering the nature of the study or seemingly suspicious were to be dropped. Myers and Jirjees (1983) attributed discovery 
to libraries with a small number of professional staff and a smaller percentage of full-time students (pp. 35-36).

Fifteen questions were posted to the Florida community colleges' digital reference sites during various times of the day, morning, afternoon, evening, late night, and weekends. Live proxies were not needed as the e-mail proxy provided a consistent and unobtrusive way to ask the question without the researcher being identified. The questions were phrased in a friendly way and a background response was created in case follow-up questions were asked by the DRSPs. In all cases, the proxy did not pressure the service provider for a prompt answer. The researcher collected all of the responses and prepared them for coding.

The responses were each coded with the institution's random number to reduce the bias of the student assessor. The code included the letter S for student or R for researcher, the random number assigned to the institution, the institutional size, and the question number, i.e., S779L14.

The researcher analyzed each response using the assessment guidelines. This review was conducted prior to the students' assessments. Without knowing how the researcher scored the responses, students from IRCC analyzed five responses each using the assessment guidelines. Each student assessor was at least 18 years of age and signed a letter of informed consent prior to assessing responses (see Appendix I). The students each received the same set of information regarding the study and their role in the study. The researcher assured the students that they were assisting with the study and not being studied themselves. The students in the presence of the researcher assessed the reference responses. The responses were presented to the student exactly as the researcher received 
them. Details identifying the DRSP and the institution were blacked out preventing disclosure.

The researcher collected all of the assessed responses and entered the data into an Excel file and a Word file for further analysis. The two assessments, done by the researcher and the IRCC student, were compared using the $t$-Test for Two Independent Samples with a significance level set at 0.05 and a confidence interval at $95 \%$ to determine if a significant difference existed between the researcher's scores and the students' scores.

The researcher contacted the library administrators at each of the community college libraries to approve an interview and to suggest the name of a DRSP to be interviewed (see Appendix D). Upon receiving the approval of the library administrator to interview one of their DRSPs, the researcher contacted the DRSP to set up the interview. Each of the DRSPs received the same information regarding the study and their role in the study through details on the informed consent form. The interview questions were asked asynchronously of the DRSP, but only after data collection for the reference inquiries had taken place. The researcher collected all of the responses and entered the data into a Word file for further analysis.

\section{Formats for Presenting Results}

Results from the unobtrusive collection of reference responses were analyzed and presented in table, figure, and narrative form. The researcher used mixed data analysis for quantitative and qualitative data to document the results (Abels, Kaske, \& White, 2002). 
The researcher and the students assessed the reference responses and the researcher presented the results in table, figure, and narrative form. The results of the $t$-Test for Two Independent Samples were also included. Results from the interviews with the DRSPs were analyzed and presented in narrative form. The narrative was presented in Chapter Four and Chapter Five. Tabular and figure presentations appeared in Chapter Four, Chapter Five, and in the Appendices. Conclusions, implications, and recommendations based on the results were presented in Chapter Five.

\section{Resources Used}

The subjects included DRSPs from Florida's community college libraries and students from IRCC. The DRSPs interviewed were from 23 of Florida's 28 community college libraries. Twenty-four library administrators authorized participation in the interview portion of the study; however, only 23 DRSPs participated. Each of the DRSPs received two consent forms, one to sign and return, and one to keep. Each of the 23 DRSPs responded to 15 interview questions. The other DRSPs were those individuals who responded to the reference questions asked through the unobtrusive portion of the study. As per Nova Southeastern University's IRB approval, their consent was not required; therefore, consent forms were not needed. The assessors included 48 students from IRCC. A maximum number of 84 assessors were needed if all 28 community college libraries answered all 15 reference questions; however, the number of students was reduced due to the number of responses received. Each of the student coders received two consent forms, one to sign and return, and one to keep. Each student assessed five separate reference responses. 
The researcher designed the reference questions, the interview questions, and the coding guidelines as instruments to collect the data for the study. A panel of six library and information science experts determined the validity and reliability of the tools. The researcher also developed all informed consent and IRB documents. The use of a computer workstation with Internet and e-mail access was required.

\section{Reliability and Validity}

To assure the reliability and validity of the instruments, the researcher studied existing instruments and comments made by authors of those instruments (Bristow \& Buechley, 1995; Bushallow-Wilbur, DeVinney, \& Whitcomb, 1996; Daniel, 2003; Duff \& Johnson, 2001; Grogan, 1992; Schilling-Eccles \& Harzbecker, 1998). The reliability of the instrument indicated, "The degree to which a test consistently measures whatever it measures" (Gay \& Airasian, p. 627; Hernon \& McClure, 1987a, p. 41). The validity of the instrument indicated, "The degree to which a test measures what it is intended to measure" (Gay \& Airasian, 2000, p. 630).

The researcher reviewed three criterions to determine the reliability of the instruments. These instruments were adapted from tests and techniques used during the past three decades of documented unobtrusive testing (Crowley \& Childers, 1971; Dyson, 1999; Hernon \& McClure, 1986; Jirjees, 1981; Kaske \& Arnold; Saxton \& Richardson, 2002). The reference questions were assessed to determine if they represented questions characteristic of community college students. The questions met the criterion because they were based on inquiries sent to the researcher's community college library or they were adapted from questions used by other researchers in a reference or information 
retrieval setting. The second criterion dealt with the coding guidelines. The researcher and the student assessors used the same coding guidelines, instructions, and training. The researcher used inter-rater reliability as the third criterion. Gay and Airasian (2000) recommended this coefficient for subjective tests, performance tests, or tests with essay type responses (p. 175). The percentage of researcher and student assessor agreement for the communication and the accuracy/satisfaction tests was $86 \%$ respectively.

The researcher determined the validity of the instruments using a panel of library and information science experts. The panel assessed the content validity of the reference questions, the interview questions, and the coding guidelines between April 3 and April 20, 2004. The panel made comments, corrections, and suggestions independent of one another. They reviewed the reference questions for clarity, suitability for the library type, level of difficulty, appropriateness for the typical undergraduate student, and representative of questions asked at community college libraries. They reviewed the interview questions for clarity, suitability for the study topic, appropriateness for the questions raised about asynchronous reference services, and representative of questions asked about reference services. They reviewed the Checklist and Scoring guidelines for clarity, suitability for the study topic, appropriateness for an assessment of reference service, and accuracy and consistency in assessment and coding. After several iterations, the researcher edited the list based on the panel's remarks and firmed up the final list of the 15 reference questions, 15 interview questions, and the coding guidelines for the study. The panel of experts found the three instruments showed content validity since they characterized the representative content. Hernon and McClure (1987b) recommended this approach. Kellehear (1993) suggested that the combination of 
unobtrusive observation and interviews "may actually increase the validity of the findings because one method may turn up findings which can be explored by the other" (p. 10).

\section{Summary}

The study was accomplished using an unobtrusive methodology to observe and evaluate the asynchronous service provided to DL students by Florida's 28 community college libraries, by assessing the responses generated from the unobtrusive portion of the study, and by interviewing the DL service providers at Florida's 28 community college libraries. Random numbers were used to protect the identity of the 28 community college libraries and their DRSPs. Chapter Three addressed the selection of the population, design of the instruments, validity and reliability of the instruments, data collection techniques, and the format for presenting the results. The unobtrusive testing methodology contributed to the assessment of the effectiveness of asynchronous reference service and to the manner by which the DRSPs treated patrons by reducing the possibility of the Hawthorne Effect (Tygett, Lawson, \& Weessies, 1996). The study results could be used to improve the accuracy of reference responses, the communication techniques between DRSPs and DL students, and the development of standards for asynchronous reference service necessary to the discipline, IHE, and DL students. 


\section{Chapter 4}

\section{Results}

\section{Analysis of the Data}

To determine the adequacy of asynchronous e-mail reference services offered to DL students at Florida's 28 community college libraries and the contribution of library staff to the students' online learning community, the researcher conducted both interviews with DRSPs within Florida's community college library system and an unobtrusive study of reference services provided to students within the digital environment. The responses provided to the researcher during the interviews, outcomes of digital reference services from the unobtrusive study, and the assessments of the unobtrusive reference responses by the researcher and by students were reported in Chapter Four. Chapter Five then addressed each of the study's research questions separately by integrating results from the interviews, the unobtrusive study, and the assessments. The integrated review enabled the researcher to analyze the DRSPs actual practices versus institutional policies or personal preferences.

The responses from interviews conducted with the digital reference service providers helped the researcher address those research questions dealing with the students' online learning environment and the DRSPs' perspectives about the type of service students should have received in a digital environment and the digital reference services each college expected to provide to students. The unobtrusive portion of the study focused on 
the research questions dealing with the accuracy and adequacy of reference service provided to the students, as well as the DRSPs' communication skills within the online environment. The assessments conducted by both the researcher and the students enabled the researcher to ascertain whether the students' responses were significantly different from the researcher's responses thereby affecting the analysis of the data.

\section{Responses from the Digital Reference Service Providers}

To seek perspective about the asynchronous service policies and procedures offered at Florida's 28 community college libraries and the perspectives of the DRSPs, the researcher sent each library administrator an e-mail requesting permission for one of their digital reference staff members to participate in a doctoral study addressing the effectiveness of asynchronous reference service for DL students. The researcher had a list of 15 questions to ask the DRSPs related to institutional policies and procedures for its digital reference services. Of the 28 administrators, 9 represented large-sized institutions, 9 represented medium-sized institutions, and 10 represented small-sized institutions. Of the 10 small-sized institutions, nine of the library administrators agreed to participate; however, only eight DRSPs participated despite repeated requests. One small-sized institution declined participation stating, "The staff member who handled the online reference services at our library is no longer with us, so we cannot help you at this time." Of the nine medium-sized institutions, eight of the library administrators agreed to participate; however, only seven DRSPs participated despite numerous requests. One library administrator never responded despite numerous requests. Of the nine large-sized institutions, eight of the library administrators agreed to participate and eight of their 
digital reference providers contributed to the study. One large-sized institution declined participation stating, "We do almost nothing with DL students and very few e-mail transactions so I do not think we can help you with your dissertation. Good luck.”

Question 1 - Does your library have written policies and procedures for AskA e-mail reference service?

When asked this question, 17 of the 23 responding DRSPs acknowledged not having policies or procedures in place. Twenty-eight institutions were invited to participate in the interview, 24 agreed to participate, and 23 participated. Three large-sized institutions, two medium-sized institutions, and one small-sized institution indicated having written policies and procedures in place for asynchronous reference service. Five DRSPs indicated adherence to the statewide "Ask a Librarian" synchronous service policies. Three DRSPs confirmed the use of existing reference policies and procedures for both traditional and asynchronous reference service. Six DRSPs either sent existing policies and procedures or referred the researcher to their respective Web sites.

Question 2 - How many people handle AskA e-mail reference questions at your library? What types of degrees do they each have? How long have they been working in libraries?

A review of the 23 responses documented 99 DRSPs in place at responding institutions. The eight responding DRSPs at the small-sized institutions identified 15 full and part-time librarians as contributing to the service. They each possessed an MLS, MLIS, or MSIS; one had a second master's degree. Their longevity of service ranged from 1 year to 27 years, with a mean of 12 years and a mode of 5 and 14 years. The seven 
responding DRSPs at the medium-sized institutions identified 36 full and part-time librarians as participating in asynchronous reference service. Of the 36 , all but one DRSP indicated earning an MLS degree. One of the 36 had only a BA degree and one had additional educational experience beyond the MLS. The longevity of service ranged from 3 years to 33 years, with a mean of 16.5 years and a mode of 10 years, excluding two respondents who provided only a range of service dates. The eight responding DRSPs at the large-sized institutions identified 48 full and part-time librarians offering asynchronous reference service. Forty-seven DRSPs had MLS degrees and one DRSP had a Masters in Instructional Systems Technology. Additionally, two DRSPs were pursuing doctoral degrees and one had a second master's degree. Their longevity of service ranged from 2 years to 37 years, with both a mean and a mode of 15 years of service, excluding two respondents who provided only a range of service dates.

\section{Question 3 - How do you train your AskA e-mail reference staff?}

Of the 23 responding DRSPs, 10 DRSPs indicated that they received their training through the statewide "Ask a Librarian" synchronous session training. The "Ask a Librarian" training included a full day of onsite training for each participating institution and periodic refresher sessions. The DRSPs used this training for both synchronous and asynchronous service. Additionally, five DRSPs indicated in-house training was provided for participation in asynchronous service, and six DRSPs offered informal training. New librarians often received in-house training on a one-to-one basis. Informal training involved discussing those questions received through digital reference amongst the DRSPs, reviewing the responses given for earlier questions, and learning the basic 
mechanics for the online forms. One DRSP commented, "We do not have any formal training. We have enough experience and we discuss our questions and responses, especially during our 6-month retreat. We came into the service knowing how to do reference." Three DRSPs reported that they received no training at all.

Question 4 - What types of questions does your AskA e-mail reference service typically receive?

When asked this question, the responses were wide-ranging; however, they fit into a variety of categories. Seventy-four percent of the 23 responding DRSPs received database access, searching, and troubleshooting inquiries. Fifty-seven percent of the DRSPs received questions regarding circulation services, such as reserve, due dates, renewals, and fines. Of the group, $44 \%$ received research or reference questions and $44 \%$ fielded barcode, password, or library card questions. Thirty-one percent of the DRSPs received public service questions about interlibrary loan, library collections, library instruction, and library handouts or questions from vendors. Twenty-six percent of the DRSPs identified online public access catalog (OPAC) questions or searches as typical. Twenty-two percent of the group received software, hardware, and Internet service provider questions. Seventeen percent of the DRSPs each received informational requests about the college and the library's policies, procedures, and hours. Thirteen percent of the DRSPs each received questions asking for citation assistance and journal assistance. Nine percent of the DRSPs typically received directional questions. Four percent of the DRSPs each received ready reference and referral questions. 
Question 5 - How does your AskA e-mail reference staff handle questions excluded from your library's policy?

From the 23 responding DRSPs, $65 \%$ indicated that they answered all questions with the exception of some question types, i.e., homework, medical advice, legal advice, extensive genealogical research, inappropriate pornographic requests, and out of state research requests. From this same group, $30 \%$ answered whatever questions they could and referred other questions to public libraries, other college or library departments, or back to the student because of the level of detail. Four percent of the DRSPs offered students only a jump-start on their research, assistance in person or on the telephone, and did not send books or articles in response to digital reference requests.

Question 6 - What types of questions do you find most appropriate for e-mail reference service?

Only $26 \%$ of the 23 responding DRSPs agreed that any question asked, other than those with pornographic intent, appropriate for e-mail reference service. Forty-eight percent of the DRSPs deemed short or ready reference questions appropriate. Twenty-six percent found database or online access questions reasonable. Twenty-six percent of the DRSPs regarded questions about library hours, library services, password, or policy questions as fitting. Seventeen percent of the DRSPs determined requests for assistance in locating additional, subject oriented, or specific class resources suitable. Thirteen percent of the DRSPs found research or searching type questions apt. Thirteen percent of the DRSPs determined database or research recommendations or referrals appropriate. Nine percent of the DRSPs deemed collection or OPAC questions appropriate. Four 
percent of the DRSPs identified citation assistance as acceptable. Four percent of the DRSPs deemed appropriate questions concerned with tutoring a student or with technological questions. Four percent of the DRSPs identified library orientation requests as appropriate to e-mail reference service.

Reasons for the disparate comments included a variety of favorable and unfavorable opinions. One DRSP service provider stated, “... online students may not have the opportunity to visit the library in person. Any contact between library staff and students is important." Another DRSP stated, "Any online contact a customer has with our library is welcome just as if the person is standing at the reference desk. The most appropriate question is whatever the needs are of the person asking." Opinions restricting the types of questions included comments, such as, "They would be better answered by the student coming into the library and asking the question." Another DRSP said, More detailed e-mail reference questions present a very unique problem because there is no guarantee that you will ever speak to that patron again. By that I mean, if you have a question in progress and the patron needs to e-mail you again at a future date, there is no guarantee that their "in progress" question will not be assigned to another librarian next time around.

Another DRSP remarked, "When a reference interview is required to determine what precisely the student needs, that is awkward and time-consuming to handle electronically." 
Question 7 - How do you assist patrons who are not included in your AskA e-mail reference policy?

Although the answers varied, the position taken by a number of DRSPs was the need to know the patrons' status to determine whether licensed resources could have been used when responding to their inquiries, especially if the DRSPs e-mailed articles to the patron. Of the 23 respondents, $61 \%$ indicated they would assist anyone who asked with the exception of those individuals with inappropriate questions; 30\% would assist all patrons, but might have referred them to another library for additional assistance; and $13 \%$ indicated that they would assist all patrons, but may provide non-residents with basic college information only.

Question 8 - How do you develop an online relationship with students who ask for help via e-mail?

The researcher collected and sorted the responses from the 23 responding DRSPs into five categories. The categories included the approach, the response, the ongoing relationship, the timeliness of the response, and the inability to develop an online relationship. Prior to detailing the responses, one comment emerged as a method of preparing digital reference providers for the experience. The DRSP stated, "Digital service providers must take an online class to get a feel for the experience."

The first category was the approach. Responses in this category focused on a beginning-to-end method of handling the student's inquiry. DRSPs believed the use of salutations, greetings, and sign-offs critical to developing an online relationship. They suggested the use of name, title, phone number for both the provider and the general 
reference desk, and the e-mail address of the provider. Many libraries sent the students' inquiries to a folder viewed by all of the providers. The DRSPs recommended the use of a personal and encouraging tone to establish rapport. One provider asked about unusual e-mail addresses and another added personal perspective into the response when warranted. Techniques included imbuing a sense of humor and a cheerful, chatty, friendly tone into the response.

The second category was the response. DRSPs recommended that responses be clear and complete, including as many options as the provider believed would answer the question. The suggested language from a number of providers was informal and easily understood by many readers. They advocated for a tone of voice that was cordial, helpful, friendly, and natural, but not canned. The response needed to include detailed steps with resources of reputable quality. The suggested closing was a request for follow-up questions and an expression of thanks for using the service.

The third category determined how the DRSP might continue developing an online rapport with the student. The answer most frequently mentioned was the use of an encouraging tone that invited additional questions or volunteered further assistance. Some DRSPs supported the use of encouragement through telephone calls, face-to-face appointments, or synchronous chat sessions. One DRSP asked students to provide feedback on the success or failure of the answer so improvements or further assistance might be offered. Another DRSP attempted to remember repeat customers by name or by question. Other DRSPs conducted follow-up e-visits to the student to inquire about the student's success. Thanking the student for his or her patronage and inviting the student to utilize the service again surfaced in their comments. 
The fourth category dealt with the timeliness of the provider's response to the student. The DRSPs mentioned a prompt reply as critical when dealing with students in the online environment. One DRSP suggested giving the student periodic updates especially if a timely response was not available.

The final category targeted negative attitudes from students. One DRSP remarked, "If we're lucky, we get a Thank you." From the responses, the inability to establish any type of relationship with the student emerged. The pervading impression garnered from some of the DRSPs was that few online encounters engaged students. One DRSP suggested that too many librarians were involved in the service thereby preventing the establishment of online relationships. "Since we have four part-time librarians and two full-time, it would be difficult to develop relationships." One DRSP intimated that the development of an online relationship with any online students had not yet happened. Another DRSP suggested that the relationship equated to a one-time interaction with a face-to-face student. "I'm not sure if I am supposed to develop a relationship, like ordering shoes online, the person has a comfort level and I'm not sure this is the same experience they get in person, it's a compromise."

Question 9 - What do you perceive is your role in developing the students' online learning environment?

Most DRSPs agreed that the library was a student support system. The role of the library and its services, both online and on campus, were critical to student success. Some librarians taught class in the online environment. They were uniquely positioned to assist students from two perspectives, that of an instructor and that of an information 
professional. For those DRSPs who had taken classes on their own or due to institutional requirements, the perspective broadened to even that of a student. One DRSP stated, "Online learning is rather intimidating. I would want to make it less daunting."

The DRSPs considered their online library services a bridge to student success. They detailed several methods of building and maintaining the bridge including teaching students how to conduct independent research, providing them information to help them get started, or introducing the students to information literacy skills. They suggested that their role was to provide timely personalized one-on-one guidance and direction in a sometimes-confusing online environment.

One DRSP considered digital reference service a portal to other college services. One DRSP likened his or her role to that of an online mentor. One DRSP commented, "Any contact is important no matter how it is done." Their role was further defined by the need to provide students with quality electronic resources in a user-friendly environment. One DRSP suggested the presentation of a usable library homepage aided in the development of the students' online learning environment.

The role of the DRSP was similar to the role of the reference librarian working with students in a face-to-face environment. DRSPs offered students service in the online environment equivalent to what their institutions offered in person. They indicated that their assistance was essential for the students' online learning success. The DRSP assisted students in learning the skills they needed to be successful in an academic environment. They guided students through the research process even though the environment was electronic. They taught students how to phrase a research question, 
select or navigate electronic tools, and evaluate results. The online environment slowed down the process.

In some cases, the DRSP suggested that they determine the level of assistance required for each question. The student might have needed help setting up a Hotmail account or navigating WebCT. The DRSPs indicated an attempt to do whatever they needed to help the student succeed in the online environment. One DRSP stated, "It's hard to know where to stop."

Question 10 - How do you learn about the student's computer skills, Internet skills, or research skills?

Most DRSPs agreed that they could not determine a student's skills in the online environment so assumptions were not always possible to make about a student's level of technological knowledge or research capabilities. Most DRSPs had an insufficient amount of time to gauge the expertise of the student. The DRSPs referred students with observable difficulties, deficiencies, or weaknesses to other college departments, classes, tutoring labs, or the library. Oftentimes, students admitted to their own shortcomings or the DRSPs asked the students about their experience with both computers and research. Based on this question, one DRSP raised a concern that the "customer's comfort and experience level with technology... may need to be formalized and addressed more often."

The DRSPs agreed that a student who located the "Ask a Librarian" Web page, input a question, and sent an e-mail had at least rudimentary computer skills. Some DRSPs indicated that a student with poor typing skills might also have poor computer skills. 
Inexperience with typing suggested the absence of either a keyboarding class or infrequent use of the computer. The DRSPs assumed that all students possessed insufficient research skills since a determination was not possible for each student. Some DRSPs suggested that students with poorly formulated research questions might point toward inadequate research skills. Poor research skills appeared more problematic to the DRSPs than inadequate computer skills. One DRSP commented that students were required to attend library instruction sessions thereby reducing both technological and research difficulties.

DRSPs provided responses to even those students with observable technological or research difficulties; however, detailed instructions and comprehensive resource information accompanied answers. One DRSP said, “...we tend to assume that the student does not know a lot about online research, and are much more likely to provide more instruction than not enough.” Some DRSPs asked the students if they understood the information provided to them. If the student offered a negative response, the DRSP sought further information about the student's skill level. The DRSP might have asked students where they had already searched for an answer to their question. At the very least, the DRSPs expected the student to say the Internet. A DRSP recommended that each e-mail message sent to the student include a comprehensive message, an invitation to ask for further assistance, and complete contact information. 
Question 11 - How do you believe the use of e-mail for reference service changed the way library staff conduct the reference interview?

The responses to this question provided an overview of the issues of time, technique, and student expectations. Many of the DRSPs indicated that e-mail provides the librarian with additional time to work on the student's question. The DRSP had the opportunity to consult with colleagues, to check multiple resources, and to send an accurate and comprehensive response. The additional time enabled an answer that had various scenarios based on the librarian's interpretation of the question. With the need to interpret different versions of the question, the DRSP often provided more information than the student needed did. The answer was detailed, succinct, comprehensive, and complete with systematic instructions.

The opposing argument was the opinion that timely reference transactions could not take place in an asynchronous environment. An interaction that went back and forth a few times delayed the resolution of the question. Many DRSPs appeared stressed by the time delay, as they believed it affected the relationship they developed with the student. "If the replies take too long you can lose the patron.” The online interaction took much longer than the face-to-face interaction. One DRSP commented,

What is amazing to me is that students don't seem to mind if it takes much longer, apparently some are more comfortable in an environment where they can be anonymous and invisible, seems less threatening online to admit they don't how to do something.

In terms of the reference interview, the DRSPs offered varied opinions. Some concluded that the asynchronous environment had not changed reference interviewing. 
Some observed the interview as an abbreviated version of the traditional reference interview. Some believed that reference became more of a guessing game causing a decline in the quality of service. One DRSP believed that the two types of service had different interview processes. "This question is like asking how has a two point foul in basketball changed where the football goal posts are placed." Another DRSP suggested that the use of e-mail eliminated the need for a traditional reference interview. Questions seeking for clarification of a request rarely arose. One DRSP stated, "I don't believe that using e-mail provides a true reference interview. The process used to answer these questions right now is not a true interview."

Most agreed that the availability of asynchronous tools for reference service challenged the reference staff. Due to the asynchronous nature of e-mail reference, librarians needed to develop a more organized approach to their reference interviewing techniques. Relying less on nonverbal cues and more on interpreting clues, the interviewing process was more instructional and dependent upon professional judgment and expertise. The DRSPs needed to request feedback from the student. One DRSP said, "The librarian needs to be able to give an easily followed step by step response which probably spills over into face to face reference which can be beneficial."

The absence of a face-to-face interaction caused more than one DRSP to comment that traditional techniques, as basic as consideration for the student, were forgotten when a quick reply was sent to the student. While DRSPs might have suggested that students did not wish to develop an online relationship with a librarian, they believed the student expected a comprehensive response from the DRSP with the first e-mail. With asynchronous reference relying more on "best guesses," "mind reading," and 
interpretative techniques, the use of an informational template might have helped the DRSP determine the needs of the student.

Question 12 - Do you survey your e-patrons to assess their satisfaction with the service?

Of the 23 responding DRSPs, $78 \%$ indicated that they did not survey their e-patrons to assess their satisfaction with digital reference. Twenty-six percent said they would or might assess service in the future. They would either design a new tool or add a question to an existing survey. Thirteen percent said they asked if students have additional questions that the DRSP might answer. Thirty-nine percent did not assess the service at all with one member of this group explaining that digital reference was just a small piece of their library service. One DRSP commented that they received thank you messages from students. Two DRSPs said that they believed the statewide "Ask a Librarian" service assessed satisfaction; however, the statewide service assessed only the synchronous service. Twenty-two percent of DRSPs indicated that they survey their epatrons using the library's annual survey, Web site evaluation tool, course evaluations, library or service satisfaction tool, automated questionnaires, or survey tool sent immediately after the transaction.

Question 13 - How does your library or college monitor your AskA e-mail service for quality?

Responses to the question about quality service resulted in a range of replies. More than $52 \%$ of the 23 responding DRSPs said that they do not monitor digital reference service for quality. One DRSP commented, “We don't. Since all of our librarians are 
professional, monitoring might be an insult." Another DRSP said, “I don’t think it makes any difference. I wouldn't stop doing it because of the quality." Thirty-nine percent reviewed answers, and in some cases, supplemented the information sent to the patron. One DRSP was not sure if the service was monitored for quality. Some institutions kept only statistics for the number of questions answered. Some DRSPs sought assistance from one another, monitored themselves, discussed the service at their meetings, or relied on the student to let them know if additional assistance was needed. One DRSP commented that the institution did not have enough staff to monitor the quality of this particular service. Another mentioned that the number of requests received was insufficient to warrant the assessment.

Question 14 - What other methods does your library staff use to judge the effectiveness of its service?

The DRSPs offered an assortment of responses; however, $26 \%$ of the 23 DRSPs used surveys as an alternate method to judge the effectiveness of its digital reference service and $30 \%$ did not use alternate methods for assessment. Thirteen percent of the group kept folders filled with messages, comments, notes of gratitude, and complaints while another $13 \%$ of the group communicated amongst the professional staff. One DRSP watched for repeat customers, talked to students in library instruction classes, and discussed the service during orientation sessions. Another DRSP spoke about pride in service being a method of assessment while another discussed tracking the number of questions asked. Only one DSRP assumed that the synchronous reports provided for the statewide "Ask a Librarian" service included asynchronous data. 
Question 15 - How should DL students receive reference assistance if library policy restricts the types of questions answered by AskA e-mail reference service?

From the group of 23 DRSPs, $78 \%$ indicated that they did not restrict the types of questions asked by students; however, they referred questions to other college departments, college help desks, educational institutions, or public libraries, when appropriate. This statistic contradicted earlier responses given by the DRSPs, thereby supporting the need for both service standards and assessment. Most DRSPs disregarded obscene, profane, or obnoxious questions. The majority of the group discussed the policy as not being restrictive so much as the student's need being unsuitable for the online environment. The DRSPs asked these students to contact the library by telephone, schedule a synchronous chat session, or explore an online tutorial. To assist students in the online environment, some libraries provided outreach to students at campuses without library services and to students in specific disciplinary areas, included handouts in college packets, and designed a usable Web site. One DRSP stated that the asynchronous service offered a starting point in the research process, but it did not supply the student with all of the materials needed for a research project. They used detailed instructions to aid these students during their initial inquiries. One DRSP indicated that e-mail reference questions were a lower priority than other reference questions.

Although the majority of the DRSPs responded that they did not restrict the types of questions asked, some comments were worth repeating.

- "Library staff should not restrict service to their own students. If a student has a legitimate question, the librarian should answer the question. Students cannot succeed if their support systems reject them. If a library restricts service, perhaps 
its staff should not offer online reference assistance. More and more students take online classes. If libraries cannot improve or augment their services, let libraries who believe in comprehensive service, conduct it. If the question is beyond the scope of the library providing the service, the library staff should intermediate a referral on the student's behalf."

- "If I have a distance learning student I will work with them to make sure that they get what they need (to the best of my ability). Even if I have to scan pages from books and email them to the person. I don't believe we should be restrictive on the email assistance to any student (especially if attending our institution) other than not doing their homework assignments for them. I've even used the local "Ask a Librarian" chat and set up a time for the student and myself to connect and work through some research issue."

- "If a library restricts the service they offer to their students then the student should use a different library. I mean to say that we believe that any student with a question is valuable to this library and we should take every measure to help find the answer that the student needs. There should be absolutely no 'restrictions' placed on the type of question a librarian will or will not answer."

- "I would like to make a point that in answering our e-mail questions, our main goal as an academic library is to teach the patrons how to find information for themselves, not find it for them. We are always here to help, but we feel that help is best given through understanding of the systems and tools available. If a student is really stuck and the question is very involved, it would be in the student's best 
interest to make a trip to the library, otherwise s/he may not be best served through only e-mail."

- "We're open to all. We do things that other libraries do not. We find it a courtesy to do extras. The quality of their questions has declined."

\section{Background Details on the Unobtrusive Study}

This study examined the adequacy of asynchronous e-mail reference services offered to DL students at Florida's 28 community college libraries and the contribution of library staff to the students' online learning community. The researcher directed unobtrusively 15 questions to DRSPs at Florida's 28 community colleges.

The researcher asked three questions during the business day between 8:00 a.m. and 12:00 noon, five questions during the business day between 12:01 p.m. and 5:00 p.m., three questions during the early evening between 5:01 p.m. and 9:00 p.m., one question during the late evening between 9:01 p.m. and 7:59 a.m., and three questions during the weekend between 5:01 p.m. on Friday and 7:59 a.m. on Monday. The researcher asked two questions on Monday, one question on Tuesday, three questions on Wednesday, four questions on Thursday, two questions on Friday, and three questions during the weekend with a question on Friday night, Saturday, and Sunday. The researcher dropped question four due to the Internet's slow response and the inability to send inquiries to no more than eight libraries within a one-hour period. This slow response compromised the collection of data during the specified time.

The researcher encountered several problems during the unobtrusive part of the study. The e-mail link on the library's page was broken for at least nine libraries forcing the 
researcher to contact a DRSP through alternate means. The researcher contacted the DRSPs through their direct library e-mail accounts, located a secondary asynchronous form on the library's Web page, or located a secondary asynchronous form from the "Ask a Librarian" link on the LINCC library catalog page. Three libraries had detailed asynchronous request forms requiring extensive personal information forcing the researcher to contact a DRSP through one of the aforementioned methods. One library offered two e-mail addresses for asynchronous service; however, both addresses were displayed incorrectly. For consistency, the researcher identified herself as a student in all cases. The DRSPs questioned the proxy's affiliation a number of times.

\section{Results of the Unobtrusive Study of Asynchronous Reference Service}

The proxy asked 14 of the 15 questions to digital reference service providers at Florida's 28 community college libraries. The researcher dropped question four due to slow Internet response time. Of a possible 392 answers, the researcher recorded 240 responses for a $61 \%$ response rate (see Table 1). The response time ranged from 6 seconds to 20 days with the proxy receiving varying percentages of the responses (see Figure 1). The researcher and student assessors indicated differences with the accuracy of the responses (see Table 2), satisfaction with the response as indicated by a willingness to return to the DRSP again, (see Table 3), additional assistance and referrals (see Table 4), and communication with the proxy (see Table 5). 
Table 1. Response Rate for 14 Questions

\begin{tabular}{lccc}
\hline Questions & Potential Replies & Response Rate & Percentage Returned \\
\hline Small & 140 & 64 & $46 \%$ \\
Medium & 126 & 85 & $67 \%$ \\
Large & 126 & 91 & $72 \%$ \\
Total & 392 & 240 & $61 \%$ \\
\hline
\end{tabular}

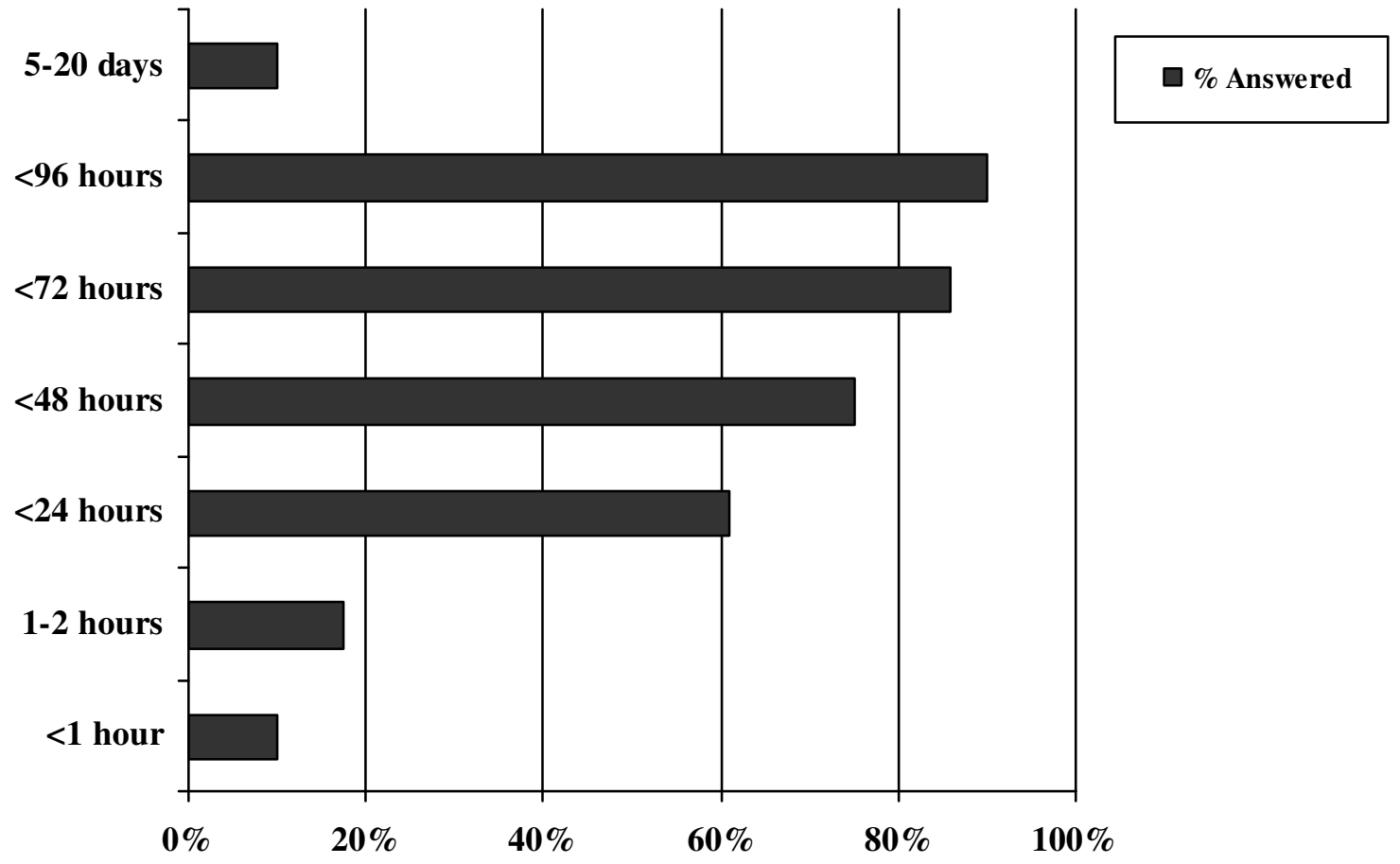

Figure 1. Response time and the percentage of questions answered. 
Table 2. Accurate Responses

\begin{tabular}{lcccc}
\hline Accuracy of Response & Researcher & 240 responses & Students & 240 responses \\
\hline Accurate w/ source & 60 & $24 \%$ & 116 & $48 \%$ \\
Accurate w/out source & 10 & $4 \%$ & 28 & $12 \%$ \\
Partly accurate w/ source & 47 & $20 \%$ & 41 & $17 \%$ \\
Partly accur w/o source & 17 & $7 \%$ & 22 & $9 \%$ \\
Inaccurate w/ source & 23 & $10 \%$ & 27 & $11 \%$ \\
Inaccurate w/out source & 8 & $3 \%$ & 18 & $8 \%$ \\
Answer > one source & 102 & $43 \%$ & 42 & $18 \%$ \\
"Don't Know" answer & 6 & $3 \%$ & 11 & $5 \%$ \\
Part/All ans w/ referral & 108 & $45 \%$ & 141 & $59 \%$ \\
No answer w/ referral & 76 & $32 \%$ & 68 & $28 \%$ \\
No answer w/o referral & 3 & $<1 \%$ & 16 & $7 \%$ \\
Source and source info & 170 & $71 \%$ & 164 & $68 \%$ \\
Source w/o source info & 7 & $3 \%$ & 48 & $20 \%$ \\
\hline
\end{tabular}

Table 3. Satisfaction Indicated by Willingness to Return to the DRSP

\begin{tabular}{lcccc}
\hline Satisfaction & Researcher & 240 responses & Students & 240 responses \\
\hline Yes & 150 & $62.50 \%$ & 139 & $58 \%$ \\
No & 90 & $37.50 \%$ & 96 & $40 \%$ \\
No response & 0 & $0 \%$ & 5 & $2 \%$ \\
\hline
\end{tabular}


Table 4. Additional Assistance and Referrals

\begin{tabular}{lcccc}
\hline \multicolumn{1}{c}{ Assistance or referral } & Researcher & 240 resp & Students & 240 resp \\
\hline DRSP acted as a go-between & 9 & $4 \%$ & 13 & $5 \%$ \\
DRSP provided no assistance & 22 & $9 \%$ & 5 & $2 \%$ \\
Recom service \& go-between & 2 & $<1 \%$ & 11 & $5 \%$ \\
Recom service w/ no assistance & 10 & $4 \%$ & 12 & $5 \%$ \\
Provided an outside referral & 5 & $2 \%$ & 0 & $0 \%$ \\
Part/All answer w/ referral & 108 & $45 \%$ & 141 & $59 \%$ \\
No answer w/ referral & 76 & $32 \%$ & 68 & $28 \%$ \\
No answer w/o referral & 3 & $<1 \%$ & 16 & $7 \%$ \\
\hline
\end{tabular}

Table 5. DRSPs' Communication with Proxy

\begin{tabular}{lcccc}
\hline \multicolumn{1}{c}{ Communication } & Researcher & 240 responses & Students & 240 responses \\
\hline Greeting used & 118 & $49 \%$ & 133 & $55 \%$ \\
Name used & 131 & $55 \%$ & 126 & $53 \%$ \\
Interest shown & 9 & $4 \%$ & 128 & $53 \%$ \\
No criticism given & 231 & $96 \%$ & 180 & $75 \%$ \\
Repeats question & 186 & $78 \%$ & 106 & $44 \%$ \\
Asks question(s) & 23 & $10 \%$ & 44 & $18 \%$ \\
Explains what to do & 193 & $80 \%$ & 124 & $52 \%$ \\
Provides steps to repeat & 122 & $51 \%$ & 141 & $59 \%$ \\
No spelling errors & 166 & $69 \%$ & 165 & $69 \%$ \\
Clear answer & 233 & $97 \%$ & 190 & $79 \%$ \\
Asks if question ans'd & 34 & $14 \%$ & 72 & $30 \%$ \\
Invites student again & 63 & $26 \%$ & 107 & $45 \%$ \\
Closing used & 117 & $49 \%$ & 145 & $60 \%$ \\
Signs name & 179 & $75 \%$ & 166 & $69 \%$ \\
Position/Title offered & 121 & $50 \%$ & 136 & $57 \%$ \\
\hline & & & & \\
\hline
\end{tabular}




\section{Response Rate}

As detailed in Chapter Three, the proxy sent each community college library the same 14 questions. The researcher dropped question four from the study due to slow Internet response time. Slow response time compromised the scheduled period for data collection from all 28 community college libraries. From the 10 small-sized institutions, the researcher recorded 64 replies from 140 potential answers for a response rate of $46 \%$. From the nine medium-sized institutions, the researcher recorded 85 replies from 126 potential answers for a response rate of $67 \%$. From the nine large-sized institutions, the researcher recorded 91 replies from 126 potential answers for a response rate of $72 \%$. In total, the proxy asked the 28 community college libraries 14 questions for 392 potential responses. Of the 392 potential replies, the researcher received 240 answers for a system-wide response rate of $61 \%$. Additionally, the number of questions answered from the small-sized institutions showed a mean of 6.5 , a median of 7.5 , a mode of 6,9 , 11, and a range of 12 . The number of questions answered from the medium-sized institutions showed a mean of 9.3 , a median of 10 , a mode of 10 , and a range of 11 . The number of questions answered from the large-sized institutions showed a mean of 10, a median of 13, a mode of 13, and a range of 12 . The number of questions answered by all 28 community college libraries showed a mean of 8.5 , a median of 9.5 , a mode of 13 , and a range of 14 .

\section{Analysis of the Answers}

For each of the following 14 questions, the researcher and students analyzed the responses provided to the proxy by the DRSP. The researcher evaluated all 240 responses 
while each of the IRCC students $(n=48)$ evaluated 5 responses each. No two students evaluated the same response; therefore, the researcher and the student had a one-to-one analysis for each response.

Both the researcher and the students assessed the communication, accuracy, satisfaction, and timeliness of the response. The satisfaction score identified the willingness of the assessor (researcher or student) to return to the DRSP for assistance. The researcher combined the accuracy score and the satisfaction score. The researcher used the $t$-Test for Two Independent Samples, with a significance level set at 0.05 and a confidence interval at $95 \%$, to determine if a significant difference existed between the researcher's scores and the students' scores.

\section{Communication Scores}

The researcher found no significant difference between the researcher's scores and the students' scores for 12 of 14 questions for communication between the DRSP and the proxy (see Table 6). The mean communication scores for all size groups assessed by the researcher and student showed that all of the DRSPs displayed some unsatisfactory communication skills (see Table 7). A score of 15 points indicated excellent online communication skills, 10-14 points indicated adequate online communication skills, 6-9 points indicated that some online communication skills were unsatisfactory, and 0-5 points indicated that online communication skills were inadequate. The researcher gave the small, medium, and large libraries each 13 scores indicating some unsatisfactory communication skills and 1 score indicating adequate online communication skills. The student gave the small libraries 8 scores indicating some unsatisfactory communication 
skills, 5 scores indicating adequate online communication skills, and 1 score indicating inadequate communication skills. The student gave the medium libraries 11 scores indicating some unsatisfactory communication skills and 3 scores indicating adequate online communication skills. The student gave the large libraries 12 scores indicating some unsatisfactory communication skills and 2 scores indicating adequate online communication skills. Not one library received a score for excellent online communication skills.

Table 6. Significance of Researcher (X) vs. Student (Y) Scores - Communication

\begin{tabular}{lccccccc}
\hline Significance & Q1 & Q2 & Q3 & Q5 & Q6 & Q7 & Q8 \\
\hline Mean X & 8.31 & 8.03 & 6.8 & 7.23 & 7.27 & 8.1 & 8.73 \\
Mean Y & 9.07 & 8.27 & 5.7 & 8 & 7.07 & 8.3 & 9.57 \\
SD X & 0.3 & 1 & 0.4 & 0.86 & 0.72 & 0.62 & 0.38 \\
SD Y & 0.71 & 0.93 & 0.91 & 0.7 & 1.14 & 1.18 & 1.53 \\
$t$-stat & -1.7 & 0.3 & 1.92 & -1.2 & 0.26 & -0.26 & 0.91 \\
DF & 3 & 4 & 3 & 3.84 & 3.39 & 3.04 & 2.24 \\
$p$-value & 0.9 & 0.61 & 0.8 & 0.85 & 0.41 & 0.59 & 0.78 \\
Sig Diff & No & No & No & No & No & No & No \\
\hline Significance & $\mathbf{Q 9}$ & $\mathbf{Q 1 0}$ & $\mathbf{Q 1 1}$ & $\mathbf{Q 1 2}$ & $\mathbf{Q 1 3}$ & $\mathbf{Q 1 4}$ & $\mathbf{Q 1 5}$ \\
\hline Mean X & 8.13 & 8.57 & 7.6 & 8.37 & 8.93 & 8.8 & 7.63 \\
Mean Y & 7.07 & 8.17 & 9.23 & 9.07 & 9.07 & 8.12 & 7.5 \\
SD X & 0.58 & 0.71 & 0.4 & 0.38 & 0.72 & 0.17 & 1.1 \\
SD Y & 0.31 & 1.04 & 1.16 & 1.51 & 0.86 & 0.61 & 0.98 \\
$t$-stat & 2.83 & 0.55 & -2.31 & -0.78 & -0.21 & 1.73 & 0.16 \\
DF & 3.04 & 3.53 & 2.47 & 2.25 & 3.88 & 2.32 & 3.95 \\
$p$-value & 0.03 & 0.31 & 0.94 & 0.74 & 0.58 & 0.1 & 0.44 \\
Sig Diff & Yes & Yes & No & No & No & No & No \\
\hline
\end{tabular}


Table 7. Communication Scores by Question/Size Researcher (R) vs. Student (S)

\begin{tabular}{ccccccccccccccc}
\hline Size & Q1 & Q2 & Q3 & Q5 & Q6 & Q7 & Q8 & Q9 & Q10 & Q11 & Q12 & Q13 & Q14 & Q15 \\
\hline Sm-R & 8.3 & 9 & 6.3 & 6.3 & 6.8 & 8.3 & 9 & 8.8 & 7.8 & 7.6 & 8.2 & 9.4 & 9 & 6.4 \\
Sm-S & 9.7 & 9.3 & 5 & 7.5 & 5.8 & 9.3 & 7.8 & 7.4 & 7 & 8 & 10.8 & 10 & 7 & 6.4 \\
Med-R & 8.6 & 7 & 7 & 8 & 8.1 & 7.4 & 8.9 & 7.8 & 8.7 & 8 & 8.8 & 9.3 & 8.7 & 8 \\
Med-S & 9.2 & 7.5 & 5.3 & 7.7 & 8 & 7 & 10.6 & 7 & 8.5 & 10.3 & 8 & 8.9 & 7.5 & 8.3 \\
Lrg-R & 8.6 & 8.1 & 7 & 7.4 & 6.9 & 8.6 & 8.3 & 7.8 & 9.2 & 7.2 & 8.1 & 8.1 & 8.7 & 8.5 \\
Lrg-S & 8.9 & 8 & 6.7 & 8.8 & 7.4 & 8.6 & 10.3 & 6.8 & 9 & 9.4 & 8.4 & 8.3 & 8.3 & 7.8 \\
Mn-R & 8.3 & 8 & 6.8 & 7.3 & 7.3 & 8.1 & 8.7 & 8.1 & 8.6 & 7.6 & 8.4 & 8.9 & 8.8 & 7.6 \\
Mn-S & 8.9 & 8.1 & 5.8 & 8 & 7.2 & 8.3 & 9.8 & 7 & 8.3 & 9.1 & 9.1 & 8.9 & 8.2 & 7.6 \\
\hline
\end{tabular}

Accuracy/Satisfaction

The mean accuracy/satisfaction scores for all size groups assessed by researcher and student showed that all of the DRSPs need to improve the accuracy of their responses to the proxy (see Table 8). The mean for the researcher's scores showed the need to improve the accuracy of the response and the mean for the students' scores showed the need to improve the accuracy of their response, but the response had redeeming features (see Table 9). Redeeming features indicated that the DRSP appeared to understand the reference process, but needed to improve the accuracy and the effectiveness of the response. A score of 10 points indicated an excellent response that was accurate, documented, and effective; 9 points indicated an average response that was accurate and effective; 8 points indicated the need to improve the accuracy of the response, but the response had redeeming features; 7 points indicated the need to improve the accuracy of the response; 3-6 points indicated the need to improve the accuracy of the response with less reliance on referrals; and 0-2 points indicated that inadequate assistance was 
provided to the proxy. The researcher scored the small libraries with a point spread of 310, the medium libraries from 3-8, and the large libraries from 3-9. The students scored the small libraries with a point spread of $3-10$, the medium libraries from $3-10$, and the large libraries from 3-9. Not one library received a score of two or below two indicating inadequate assistance.

Table 8. Significance of Researcher (X) vs. Student (Y) Scores - Accuracy/Satisfaction

\begin{tabular}{lccccccc}
\hline Significance & Q1 & Q2 & Q3 & Q5 & Q6 & Q7 & Q8 \\
\hline Mean X & 6.63 & 4.7 & 6.93 & 5.23 & 6.27 & 6 & 6.2 \\
Mean Y & 9.2 & 5.63 & 6.93 & 6.57 & 6.73 & 8 & 9.17 \\
SD X & 1.21 & 0.75 & 0.4 & 0.95 & 0.55 & 0.78 & 0.72 \\
SD Y & 1.61 & 1.33 & 1.53 & 0.21 & 0.23 & 0.72 & 0.67 \\
$t$-stat & -2.2 & -1.1 & 0 & -2.4 & -1.4 & -2.44 & -5.2 \\
DF & 3.71 & 3.17 & 2.28 & 2.19 & 2.68 & 3.97 & 3.97 \\
$p$-value & 0.95 & 0.82 & 0.5 & 0.94 & 0.86 & 0.96 & 1 \\
Sig Diff & No & No & No & No & No & No & Yes \\
\hline Significance & $\mathbf{Q 9}$ & $\mathbf{Q 1 0}$ & $\mathbf{Q 1 1}$ & $\mathbf{Q 1 2}$ & $\mathbf{Q 1 3}$ & $\mathbf{Q 1 4}$ & $\mathbf{Q 1 5}$ \\
\hline Mean X & 4.33 & 4.7 & 6.67 & 7.97 & 5.1 & 8.77 & 8.37 \\
Mean Y & 5.73 & 6.57 & 7.7 & 7.37 & 5.9 & 7.53 & 8.23 \\
SD X & 1.53 & 1.35 & 0.31 & 0.64 & 0.5 & 0.87 & 1 \\
SD Y & 0.91 & 2 & 0.17 & 0.21 & 0.98 & 0.68 & 1.64 \\
$t$-stat & 1.36 & -1.3 & -5.1 & 1.54 & -1.25 & 1.93 & 0.12 \\
DF & 3.25 & 3.51 & 3.17 & 2.41 & 2.97 & 3.77 & 3.3 \\
$p$-value & 0.87 & 0.87 & 0.99 & 0.12 & 0.85 & 0.07 & 0.46 \\
Sig Diff & No & No & Yes & No & No & No & No \\
\hline
\end{tabular}


Table 9. Accuracy/Satisfaction Scores by Question/Size Researcher (R) vs. Student (S)

\begin{tabular}{lccccccc}
\hline Significance & Q1 & Q2 & Q3 & Q5 & Q6 & Q7 & Q8 \\
\hline Sm-R & 8 & 4 & 6.5 & 4.3 & 6.8 & 6.8 & 7 \\
Sm-S & 8.7 & 6.3 & 6 & 6.8 & 7 & 7 & 9.5 \\
Med-R & 6.2 & 5.5 & 7.3 & 6.2 & 6.3 & 5.4 & 6 \\
Med-S & 11 & 6.5 & 7.8 & 6.5 & 6.6 & 8 & 9.6 \\
Lrg-R & 5.7 & 4.6 & 7 & 5.2 & 5.7 & 6.7 & 5.6 \\
Lrg-S & 7.9 & 4.1 & 6.1 & 6.4 & 6.6 & 8.4 & 8.4 \\
Mean-R & 6.3 & 4.8 & 6.8 & 5.3 & 6.2 & 6.3 & 6.1 \\
Mean-S & 9.1 & 5.8 & 5.8 & 6.5 & 6.7 & 7.9 & 9.1 \\
\hline Significance & $\mathbf{Q 9}$ & $\mathbf{Q 1 0}$ & $\mathbf{Q 1 1}$ & $\mathbf{Q 1 2}$ & $\mathbf{Q 1 3}$ & $\mathbf{Q 1 4}$ & $\mathbf{Q 1 5}$ \\
\hline Sm-R & 2.6 & 3.3 & 6.4 & 8.7 & 5.6 & 9.5 & 9.4 \\
Sm-S & 6.4 & 4.5 & 7.8 & 7.3 & 5.6 & 8.3 & 7.6 \\
Med-R & 5.5 & 4.8 & 7 & 7.5 & 5.1 & 7.8 & 7.4 \\
Med-S & 4.7 & 8.5 & 7.5 & 7.2 & 7 & 7.3 & 10.1 \\
Lrg-R & 4.9 & 6 & 6.6 & 7.7 & 4.6 & 9 & 8.3 \\
Lrg-S & 6.1 & 6.7 & 7.8 & 7.6 & 5.1 & 7 & 7 \\
Mean-R & 4.5 & 4.9 & 6.6 & 7.9 & 5.1 & 8.8 & 8.3 \\
Mean-S & 5.7 & 6.8 & 7.7 & 7.4 & 5.9 & 7.5 & 8.6 \\
\hline
\end{tabular}

\section{Timeliness Scores}

In terms of the timeliness of the response, the researcher determined one score for the time spent on the task and one score for the business hours spent on the task. The score for the business hours subtracted hours between 10:00 p.m. and 8:00 a.m. on Monday through Thursday evenings and hours between 5:00 p.m. on Friday and 8:00 a.m. on Monday morning. A score of 11 and below suggested reviewing digital reference processes for improving turn-around time.

In terms of timeliness, the mean for all size groups in terms of hours showed an average response time, but the mean for all size groups in terms of business hours only 
showed an above average response time (see Table 10). A score of 12-14 points indicated an above average response time, $8-11$ points an average response time, 5-7 points a below average response time, and 0 points an inadequate and unacceptable response time. For scores $0-11$ points indicated a need to review digital reference processes for improving turn-around times. Not one library received a score for excellent response time in either time or business hours only. In terms of improving turn-around time for responses, small libraries needed to review digital reference processes for 12 of the 14 questions answered and for 4 of the 14 questions answered during business hours only; medium libraries for 9 of the 14 questions answered and 1 of the 14 questions answered during business hours only; and large libraries for 9 of the questions answered and none of the 14 questions answered during business hours only.

\section{Response Rate}

The Florida community college system had 10 small, 9 medium, and 9 large colleges. The table represented the total number of community college libraries that responded to each question in total and by size, the number of questions that either failed to reach the DRSPs or could not be asked due to suspicion by college size, and the percentage of libraries that answered the question in total and by size (see Table 11). DRSPs who questioned the proxy's personal e-mail address, the proxy's use of a personal e-mail address rather than the institutional-issued e-mail address, the proxy's affiliation, and the number of recent e-mail inquiries from unusual e-mail addresses were dropped. Eight questions had response rates in the 50\% range, five questions in the $60 \%$ range, and one 
question in the $70 \%$ range. While no questions fell below the $50 \%$ range, none reached higher than a $75 \%$ response rate.

Table 10. Timeliness Scores by Question/Size Actual Time vs. Business Hours (B)

\begin{tabular}{lccccccc}
\hline \multicolumn{1}{c}{ Size } & Q1 & Q2 & Q3 & Q5 & Q6 & Q7 & Q8 \\
\hline Sm & 10 & 8 & 10 & 8 & 6.7 & 12.8 & 9.8 \\
Sm-B & 12.7 & 13 & 13.5 & 10 & 12 & 13.3 & 12.3 \\
Med & 10 & 8.7 & 11.7 & 6 & 8 & 12.6 & 12.7 \\
Med-B & 13 & 12.3 & 14 & 8.7 & 12.4 & 13.8 & 14 \\
Lrg & 10.4 & 10 & 12.3 & 8.6 & 9.1 & 12.7 & 13.6 \\
Lrg-B & 13.3 & 12.6 & 14.3 & 11.8 & 12.6 & 13 & 14.6 \\
Mean & 10.2 & 9.1 & 11.5 & 7.7 & 8 & 12.7 & 12.4 \\
Mean-B & 13.1 & 12.6 & 14 & 9.4 & 12.3 & 13.3 & 13.8 \\
\hline \multicolumn{1}{c}{ Size } & $\mathbf{Q 9}$ & $\mathbf{Q 1 0}$ & $\mathbf{Q 1 1}$ & $\mathbf{Q 1 2}$ & $\mathbf{Q 1 3}$ & $\mathbf{Q 1 4}$ & $\mathbf{Q 1 5}$ \\
\hline Sm & 7 & 8.9 & 7.4 & 9.2 & 13.8 & 6.3 & 7.2 \\
Sm-B & 8.8 & 9.5 & 10.4 & 11.3 & 14.2 & 14 & 15 \\
Med & 12.7 & 8.7 & 10.8 & 10.7 & 13 & 6.8 & 7.9 \\
Med-B & 13.8 & 14 & 14 & 13.5 & 13.6 & 14.5 & 14.1 \\
Lrg & 12.6 & 9.2 & 9.2 & 11 & 11.1 & 8.9 & 7.3 \\
Lrg-B & 13.6 & 13 & 13.6 & 13.7 & 12.1 & 14.3 & 14 \\
Mean & 11.2 & 8.6 & 9 & 10.3 & 12.5 & 7.4 & 8.1 \\
Mean-B & 12.4 & 11.6 & 12.6 & 12.9 & 13.2 & 14.3 & 14.4 \\
\hline
\end{tabular}


Table 11. Response Rate to Questions by Library Size

\begin{tabular}{|c|c|c|c|c|c|c|c|c|c|c|c|c|c|c|}
\hline Size & Q1 & Q2 & Q3 & Q5 & Q6 & Q7 & Q8 & Q9 & Q10 & Q11 & Q12 & Q13 & Q14 & Q15 \\
\hline Tot CC Resp & 15 & 16 & 17 & 15 & 21 & 16 & 18 & 19 & 16 & 14 & 19 & 19 & 19 & 16 \\
\hline Tot Sm Resp & 3 & 3 & 4 & 4 & 6 & 4 & 4 & 5 & 4 & 5 & 6 & 5 & 6 & 5 \\
\hline Tot Sm Fail & 2 & 2 & 2 & 1 & 0 & 0 & 1 & 0 & 0 & 0 & 0 & 0 & 0 & 0 \\
\hline Tot Sm Susp & 0 & 0 & 0 & 0 & 0 & 0 & 0 & 0 & 0 & 0 & 0 & 0 & 0 & 1 \\
\hline Sm Resp \% & 30 & 30 & 40 & 40 & 60 & 60 & 40 & 50 & 40 & 50 & 60 & 50 & 60 & 50 \\
\hline Tot Med Resp & 5 & 6 & 6 & 6 & 8 & 5 & 7 & 6 & 6 & 4 & 6 & 7 & 6 & 7 \\
\hline Tot Med Fail & 0 & 0 & 0 & 0 & 0 & 0 & 0 & 0 & 0 & 0 & 0 & 0 & 0 & 0 \\
\hline Tot Med Susp & 0 & 0 & 0 & 0 & 0 & 0 & 0 & 0 & 0 & 0 & 1 & 1 & 1 & 1 \\
\hline Med Resp \% & 56 & 67 & 67 & 67 & 89 & 56 & 78 & 67 & 67 & 44 & 67 & 78 & 67 & 78 \\
\hline Tot Lrg Resp & 7 & 7 & 7 & 5 & 7 & 7 & 7 & 8 & 6 & 5 & 7 & 7 & 7 & 4 \\
\hline Tot Lrg Fail & 0 & 0 & 0 & 0 & 0 & 0 & 0 & 0 & 0 & 0 & 0 & 0 & 0 & 0 \\
\hline Tot Lrg Susp & 0 & 0 & 0 & 0 & 0 & 0 & 0 & 0 & 1 & 1 & 1 & 1 & 1 & 2 \\
\hline Lrg Resp \% & 78 & 78 & 78 & 56 & 78 & 78 & 78 & 89 & 67 & 56 & 78 & 78 & 78 & 44 \\
\hline Tot Resp \% & 54.7 & 58 & 57 & 54 & 75 & 57 & 64 & 68 & 57 & 50 & 68 & 68 & 68 & 57 \\
\hline
\end{tabular}

\section{Question 1}

$\mathrm{Hi}$, I have to write a short paper on somebody famous. I want to do it on the female athlete who died last year. What's her name? What did she play again? Thx.

Known primarily for playing professional tennis, Althea Gibson, who died September 28, 2003, also played professional golf. Before she entered professional sports, she played basketball, paddle tennis, and tennis. 


\section{Researcher's Observations - Small-Sized Institutions}

Three of the 10 DRSPs responded. They all correctly identified Althea Gibson as the athlete, but only one DRSP asked the proxy to verify the suggested name. One DRSP provided tennis as the primary sport, but all three DRSPs either recommended or sent biographical articles from Biography Resource Center, Biography Reference Bank, and American National Biography databases. The American National Biography database had no information on Althea Gibson. One DRSP suggested that the proxy conduct a Google search for Althea Gibson; however, a search resulted in more than 29,000 hits. The number of results might appear staggering to a student with poor research skills; however, the DRSP directed the proxy adequately with the total response. One DRSP recommended two book titles, complete with bibliographic details, and sent four newspaper articles from the Custom Newspaper database.

Students' Comments- - Small-Sized Institutions

- "It took too long."

- "Friendly"

- "Very nice \& helpful"

\section{Researcher's Observations - Medium-Sized Institutions}

Five of the nine DRSPs responded. Four identified Althea Gibson as the athlete; however, they only mentioned the tennis connection. One DRSP asked the proxy to assist in narrowing down the possibilities by identifying the athlete's sport or country; thereby, repeating one of the proxy's own questions. One DRSP asked for confirmation on Gibson 
as the athlete. One DRSP invited the proxy to call or visit the library to learn more about the athlete prior to assisting the proxy in finding resources. Only one of the five DRSPs failed to include source information to aid the proxy. Three DRSPs suggested the Biography Resource Center database to the proxy.

\section{Students' Comments - Medium-Sized Institutions}

- "Very nice!"

- "Not bad great job"

\section{Researcher's Observations - Large-Sized Institutions}

Seven of the nine DRSPs responded. They identified Althea Gibson as the athlete although tennis was the only sport identified in their short answers. One DRSP first suggested Florence Griffith Joyner although she died in 1998, not 2003. The proxy added African American as the athlete's race to help limit the possibilities. Two DRSPs omitted source information from their responses. One DRSP recommended searching for books. Three DRSPs suggested or sent Web sources, specifically from Google, ESPN, ${ }^{46}$ WorldHistory, ${ }^{47}$ and Information Blast. ${ }^{48}$ Four DRSPs suggested the Biography Resource Center database and one recommended the Academic Search Premier database.

\footnotetext{
${ }^{46} \mathrm{http}: / /$ espn.go.com

${ }^{47} \mathrm{http}: / /$ www.worldhistory.com

${ }^{48} \mathrm{http}: / / \mathrm{www}$.informationblast.com
} 


\section{Students' Comments - Large-Sized Institutions}

- "I think she wanted to clarify the athlete before she did the research on it. I was under the impression that when the student told her that was the woman she was thinking about that the librarian would have helped more."

- "Very helpful. Seemed to care about the girls [sic] question"

\section{Researcher's Observations - All Institutions}

The Biography Resource Center database emerged as the predominant resource recommended by 9 of the 15 DRSPs. In total, four DRSPs neglected to include information regarding Web locations for LINCC, the online public access catalog, or the suggested databases. Thirteen DRSPs failed to provide information about obtaining a library barcode or password. Four DRSPs overlooked the opportunity to include information regarding logging in to the suggested databases. Five DRSPs also omitted instructions for searching the databases or LINCC. The instructions provided to the proxy by one DRSP were incomprehensible to an inexperienced searcher. Only two DRSPs provided instructions for searching the Biography Resource Center database.

\section{Question 2}

Can you help me set up a reference using APA? The article is called Predicting Serial Killers' Home Base and David Kanter wrote it. I don't know how to set it up. Thank you very much. 
The answer to the question was one of the following citations depending upon whether the article appeared in electronic or paper format.

Canter, D., Coffey, T., Huntley, M., \& Missen, C. (2000). Predicting serial killers'

home base using a decision support system. Journal of Quantitative

Criminology, 16, 457-478.

Canter, D., Coffey, T., Huntley, M., \& Missen, C. (2000). Predicting serial killers'

home base using a decision support system. Journal of Quantitative

Criminology, 16, 457-478. Retrieved date, from Academic Search Premier

database.

\section{Researcher's Observations - Small-Sized Institutions}

Three of the 10 DRSPs responded. They referred the proxy to the college library to use the Publication Manual of the American Psychological Association or to ask a librarian for guidance in using the Manual. One of the DRSPs recommended the Citation Machine -- The Landmark Project ${ }^{49}$ interactive Web site. While the site referred the user to the $5^{\text {th }}$ edition of the Publication Manual of the American Psychological Association, the user had the option of inputting reference information into the online generator for an APA or an MLA citation. The generator created an electronic or traditional citation based on the information entered by the user. If the user failed to capitalize necessary parts of the citation or spells words incorrectly, the citator did not flag the error. The citator also omitted the punctuation mark after the article title. Only one of the DRSPs identified the complete online citation for the proxy, but shared an incorrectly structured APA citation

${ }^{49} \mathrm{http}: / /$ citationmachine.net 
with its own set of errors. Additionally, this DRSP referred the proxy to an APA style guide on the library's Web site; however, the guide also had capitalization errors and punctuation errors.

Students' Comments - Small-Sized Institutions

- $\quad$ Students offered no comments for question 2.

\section{Researcher's Observations - Medium-Sized Institutions}

Six of the nine DRSPs responded. Five referred the proxy to either an APA style guide on the DRSP's library Web site or another educational Web site. These guides had capitalization errors, style errors, punctuation errors, or other examples besides the one needed. One DRSP suggested a Web site that offered the proxy a choice of 10 APA style sites. Two DRSPs recommended an educational Web site that linked the proxy to a guide that offered an accurate example of a journal article paginated by volume. Only one DRSP suggested a Google search for "APA style examples," which resulted in 239 hits from a variety of domains. The student could not know if a Web site offered examples formatted correctly without further assistance from the DRSP.

Two DRSPs mentioned online citation generators. The Citation Machine -- The Landmark Project, ${ }^{50}$ mentioned previously, and the Style Wizard. ${ }^{51}$ The Wizard allowed the user to select the type of work to be cited; however, the tool lacked an option for a database citation. A disclaimer on the site indicated that information incorrectly input

\footnotetext{
${ }^{50}$ http://citationmachine.net

${ }^{51} \mathrm{http}: / /$ www.stylewizard.com
} 
resulted in an inaccurate citation. Although the tool offered the proxy excellent prompts, it also had some punctuation errors.

One DRSP recommended the use of the APA's ${ }^{52}$ Web site for electronic resources. The site provided an accurate example of a journal article written by five authors retrieved from an online database. Only two DRSPs suggested the Publication Manual of the American Psychological Association, available at the college's library. Two DRSPs offered suggestions for citing the article. One of these two DRSPs told the proxy that the citation lacked information, but sent the proxy the missing information in a subsequent email. The examples provided to the proxy had punctuation errors, capitalization errors, and missing information.

Students' Comments - Medium-Sized Institutions

- Students offered no comments for question 2.

\section{Researcher's Observations - Large-Sized Institutions}

Four of the seven responding DRSPs referred the proxy to either an APA style guide on the DRSP's library Web site or another educational Web site. These guides had capitalization or punctuation errors, showed journals paginated by issue, or detailed other examples besides the one needed. One of these guides suggested the use of the APA Manual for further guidelines. One library Web site offered the proxy a list of 15 possible choices dealing with the documentation of sources. Only one of the four DRSPs directed the proxy to an accurate APA Web-based guide found on the DRSP's library Web site.

52 http://www.apa.org 
This site offered details about the difference between a traditional source and an online source.

Only one of the DRSPs located the missing citation information and attempted to construct the proxy's citation. The DRSP's examples neglected to include all the components needed for an accurate citation and showed punctuation errors. The DRSP also failed to mention the possibility of the article being retrieved from an online database. The DRSP cited the $9^{\text {th }}$ edition of Writing Research Papers (1998) as the resource used to answer the proxy's question. Two DRSPs asked the proxy for complete source information before providing additional assistance, including the need to know the name of the online database. One of the seven DRSPs mentioned that each of the campus libraries owned a copy of the APA Manual should the proxy want to seek information that was more detailed.

\section{Students' Comments - Large-Sized Institutions}

- Students offered no comments for question 2.

\section{Researcher's Observations - All Institutions}

Library-generated APA Web-based guides emerged as the predominant resource recommended by 8 of the 16 DRSPs. Only 6 of the 16 DRSPs suggested using the Publication Manual of the American Psychological Association. Three of the 16 DRSPs verified the incomplete citation although a Web search, the journal's Web site, or an online database search provided the missing information. The proxy misspelled the author's name, but provided sufficient information to retrieve the reference details. The 
majority of the DRSPs failed to alert the proxy to the misspelling of the author's name. A number of the tools or guides to which the DRSPs referred the proxy had formatting errors. None of the DRSPs offered to review the proxy's citation after he or she assembled it. The DRSPs also had difficulty identifying the difference between periodical and journal articles, as well as a journal paginated by volume and a journal paginated by issue. Only one of the DRSPs reminded the proxy that online or traditional citations appeared differently.

\section{Question 3}

Does the library have a copy of Lucky Jim on video? I really need to watch it. Keep me posted. Thanks.

A LINCC search showed that none of the community college libraries in Florida owned a copy of Lucky Jim on video; however, one academic and one public library within Florida owned the video. One of these two libraries loaned the video through interlibrary loan.

\section{Researcher's Observations - Small-Sized Institutions}

Four of the 10 responding DRSPs indicated that their libraries did not have the video, although one library owned the book. Two of these DRSPs checked the statewide LINCC system and indicated the same information. Two DRSPs referred the proxy to the local public library or a larger library system to check their holdings. Two DRSPs checked the local holdings and indicated that the video was not available. One DRSP referred the 
proxy to Amazon $^{53}$ to consider purchasing the video. One DRSP did not offer any referrals at all.

Students' Comments - Small-Sized Institutions

- $\quad$ Students offered no comments for question 3.

Researcher's Observations - Medium-Sized Institutions

Six of the nine responding DRSPs indicated that the library did not have the video, although three libraries owned the novel. Two DRSPs referred the proxy to LINCC for verification. Three DRSPs checked local county libraries and these libraries did not own the video although one library owned the audiocassette. One DRSP checked the local state university with no luck; however, a full search of all the university libraries would have resulted in a successful search. One DRSP referred the proxy to Amazon $^{54}$ for a potential purchase and another recommended that the proxy speak to the course instructor.

Students' Comments - Medium-Sized Institutions

- Students offered no comments for question 3.

Researcher's Observations - Large-Sized Institutions

Seven of the nine DRSPs indicated that their respective community college libraries did not have the video. One DRSP conducted a statewide search of LINCC with no

\footnotetext{
${ }^{53} \mathrm{http}: / /$ www.amazon.com
}

${ }^{54} \mathrm{http}$ ://www.amazon.com 
success. Two DRSPs indicated owning the novel. One DRSP provided the proxy with information on searching LINCC. One DRSP recommended contacting the library directly since the local university houses videotapes for its own courses and perhaps one of those videos matched the proxy's need, but the title was not listed in the catalog. Four DRSPs checked local county library systems with no success, although one of the public libraries owned the novel. One DRSP referred the proxy to the local county library and provided a telephone number. One DRSP referred the proxy to his or her local video stores to check for availability of the title. One librarian provided the proxy with information on the library barcode and PIN to access the library's databases.

\section{Students' Comments - Large-Sized Institutions}

- "Seemed very insightful"

- "Good searching"

- “This librarian didn't seem to care to help the student. I wouldn't pick her to help me."

- "It took him 72 hours to say no."

- "Not friendly"

\section{Researcher's Observations - All Institutions}

LINCC, the online catalog of Florida's community college library system, emerged as the predominant resource consulted by 17 of the 17 DRSPs. None of the libraries within the LINCC system owned the videotape; however, 15 of the 17 DRSPs failed to suggest interlibrary loan as an option. One of the 15 DRSPs indicated conducting a search of 
LINCC, the "statewide community college network for Interlibrary loans." One DRSP mentioned it, but squelched the idea because "most libraries do not lend videos to other library because they get messed up so easily." Another DRSP said,

Under normal circumstances, we are able to obtain materials for students via what we call Interlibrary Loan. We can ask libraries throughout the country to loan us materials if they are available. However, in the case of video tapes and DVDs most libraries will not send them via Interlibrary Loan because the risk of damage to them in the mail is great.

An offer was not made to attempt to secure the videotape through interlibrary loan, yet the researcher obtained the videotape from a Florida library for use by a legitimate student.

\section{Question 4}

The researcher dropped question four due to the Internet's slow response and the inability to send inquiries to no more than eight libraries within a one-hour period. MyDoom worm slowed online access and rendered many outgoing asynchronous requests undeliverable. The slow response time compromised the data collection period.

\section{Question 5}

What was the inflation rate in 1980 for tobacco products? What's the current rate? Need them for economics class project. Appreciate your time.

The inflation rate for tobacco products in 1980 was 72.0 and the rate in 2004 was 473.3 for April 2004, 473.5 for May 2004, and 476.0 for June 2004, as confirmed by data 
from the Statistical Abstract of the United States, U.S. Department of Labor's Bureau of Labor Statistics ${ }^{55}$ Web page, the CPI Detailed Report, and an e-mail from an economist with the CPI (S. Gibson, personal communication, October 25, 2004).

\section{Researcher's Observations - Small-Sized Institutions}

Four of the 10 DRSPs responded. Three DRSPs used federal government resources to answer the question. One DRSP correctly identified one of the sources as the Statistical Abstract of the United States, but provided a figure based on 1980. This DRSP also neglected both to include the current statistic and source(s) to locate the current figure. Another DRSP sent a response alerting the proxy to an attached table. While this DRSP forgot to attach the table, the proxy received specific instructions on retrieving the data from the Bureau of Labor Statistics ${ }^{56}$ Web site. The third DRSP referred the proxy to the Consumer Price Index ${ }^{57}$ Web site. The Web site offered four screens listing options; however, the DRSP neglected to include instructions to guide the proxy. One of the three DRSPs also sent links to a Federal Reserve Bank of New York report ${ }^{58}$ dealing with inflation and sent an article from Tobacco Control dealing with the marketing of tobacco. Neither article provided the inflation information requested by the proxy.

The fourth DRSP took more than six days to respond to the proxy with the first contact asking clarifying questions about the request. "What do you mean by the inflation rate for tobacco products? Are you asking how much the price went up in a year? If so,

\footnotetext{
${ }^{55} \mathrm{http}: / /$ bls.gov

${ }^{56} \mathrm{http}: / /$ bls.gov

${ }^{57} \mathrm{http}: / /$ bls.gov/cpi/home.htm

${ }^{58} \mathrm{http}: / /$ www.newyorkfed.org/research/staff_reports/sr173.pdf
} 
do you want to differentiate between the producer cost and the taxes?" The DRSP did not suggest any resources to the proxy.

Students' Comments - Small-Sized Institutions

- "Not very informative"

Researcher's Observations - Medium-Sized Institutions

Seven of the nine DRSPs responded. Four of the seven DRSPs used federal government resources to answer the inquiry. One DRSP provided an accurate answer to both the historic and the current inquiry, but also suggested the Bureau of Labor Statistics $C P I^{59} \mathrm{Web}$ site. The other three DRSPS referred the proxy to various parts of the Bureau's Web page, including the tables section of the Bureau of Labor Statistics CPI Web site, the Archived Consumer Price Index Detailed Report Tables, ${ }^{60}$ and the News ${ }^{61}$ release. The Web site offered four screens of options, but the proxy received no instructions for navigating the site. One of the DRSPs referred the proxy to the library to utilize older issues of the CPI Detailed Report for the 1980 figure despite the availability of an online archival tool.

Additionally, two of these four DRSPs provided a link to a 14-page article written by three economists from the December 1994 issue of the Monthly Labor Review. ${ }^{62}$ While the article dealt with tobacco prices between 1980 and 1994, it did not include the CPI for 1980. One of the DRSPs also e-mailed two articles to supplement the proxy's request.

\footnotetext{
${ }^{59} \mathrm{http}: / / \mathrm{www} . b l s . g o v / \mathrm{cpi} / \mathrm{home} \cdot \mathrm{htm}$

${ }^{60} \mathrm{http}: / / \mathrm{bls} . g o v / \mathrm{cpi} / \mathrm{cpi}$ dr.htm

${ }^{61} \mathrm{http}: / / \mathrm{bls}$. gov/cpi/home.htm\#news

${ }^{62} \mathrm{http}: / /$ www.bls.gov/opub/mlr/1994/12/artlfull.pdf
} 
Neither article mentioned the 1980 inflation rate and one article had missing pages. One of the DRSPs also suggested that the proxy conduct a Google search for "inflation rate in 1980 for tobacco products" to locate Web sites with statistical information; however, the initial result pages contained international statistics or the aforementioned articles.

The fifth DRSP used a 2003 edition of the World Almanac and Book of Facts to locate the information. Instead of a 1980 and 2004 figure, the DRSP provided a 1970 and a 2001 figure. The Almanac listed the U.S. Department of Labor's BLS as the source for the CPI figures; however, the DRSP neither used the source information nor shared it with the proxy. The sixth DRSP offered no answer because, "We do not conduct research on questioner's behalf, but try instead to guide them to sources they can use to find the answer." The referral suggested government Web sites accessed through a Google search of "inflation rate" and "tobacco." The search resulted in more than 91,000 hits with the first 10 highlighting countries other than the United States. The seventh medium-sized college library automatically generated a response indicating the DRSP answered most questions within 24 hours with the exception of weekend requests, which may take longer. The proxy received no other response from this library.

\section{Students' Comments - Medium-Sized Institutions}

- "Took to [sic] long"

- "But she didn't find that answer but maybe she'll help on another"

- "Very helpful, seemed willing to help student."

- "Librarian didn't seem interested in answering the students [sic] question." 
Researcher's Observations - Large-Sized Institutions

Five of the nine DRSPs responded. Two DRSPs used the Statistical Abstract of the United States. One DRSP used a 1987 version, which listed the 1980 rate as 214.5 rather than 72.0. This DRSP correctly identified the current rate by using the BLS ${ }^{63}$ Web site. The other DRSP used the online version of the Abstract; however, the table did not include data more recent than 2002. The DRSP neglected to refer the proxy to the source where current statistics were available.

The third DRSP provided the proxy with the name of the resource, but not the Web address in the e-mail. The proxy received no instructions for using the Web site. The DRSP also suggested a Google search for inflation rate "tobacco products" resulting in more than 53,000 hits with the first page displaying Canadian and European data, school lessons, and smokeless tobacco products. The DRSP also suggested a 14-page article written by three economists from the December 1994 issue of the Monthly Labor Review. ${ }^{64}$ While the article dealt with tobacco prices between 1980 and 1994, it did not include the CPI for 1980. This DRSP sent a second e-mail with three suggested Web sites. The first Web site was for tobacco prices from the Philippines' National Statistical Coordination Board. ${ }^{65}$ The second site was for an article on German tobacco prices in the Macao publication, AMCM/GEE International Economic Review. ${ }^{66}$ The third site was for the U.S. ERS/USDA Briefing Room ${ }^{67}$ for tobacco. The site contained two pages of linked documents yet the DRSP sent no instructions. The researcher checked more than seven

\footnotetext{
${ }^{63} \mathrm{http}: / / w w w . b l s . g o v$

${ }^{64} \mathrm{http}: / /$ www.bls.gov/opub/mlr/1994/12/art1full.pdf

${ }^{65} \mathrm{http}: / /$ www.nscb.gov.ph/secstat/d_price.asp

$66 \mathrm{http}: / /$ www.amcm.gov.mo

${ }^{67} \mathrm{http}: / /$ www.ers.usda.gov/Briefing/Tobacco
} 
links before finding the U.S. Tobacco Statistics. The proxy needed to review the README.TXT and the Help files to learn about uploading tables and to locate the correct table. The correct answer for 1980 was located in Table 48, which listed the CPI for the years 1940-1995.

The fourth DRSP referred the proxy to the May 2004 issue of the U.S. Department of Agriculture's circular titled Tobacco: World Markets and Trade. ${ }^{68}$ Of the document's 17 tables, none discussed inflation, CPI, or prices. The DRSP disregarded the request for the 1980 data.

After nearly seven days, the fifth DRSP responded by saying, "We were unable to find any source containing the rate of inflation for tobacco products." The DRSP suggested the Web site for the Agricultural Statistics Database, ${ }^{69}$ which provided month-by-month prices used to calculate the inflation rate. No instructions were included on how to calculate the inflation rate nor were any resources included that might help the proxy to do so. The DRSP indicated that the proxy could use the same database to determine the 2004 figure; however, the database had no 2004 data.

Students' Comments - Large-Sized Institutions

- "No real help, just a source that was referred to."

\section{Researcher's Observations - All Institutions}

Resources from the U.S. Department of Labor's BLS emerged as the predominant federal agency consulted by 9 of the 15 responding DRSPs. The Statistical Abstract of

\footnotetext{
${ }^{68} \mathrm{http}: / / \mathrm{www}$. fas.usda.gov/tobacco/circular/2004/052004/index.htm

${ }^{69} \mathrm{http}: / /$ www.nass.usda.gov/QuickStats
} 
the United States provided some historic data, but only three DRSPs considered it. Was the response rate low due to the more difficult nature of the question? Did a statistical question cause anxiety among the DRSPs? Google searches, Web sites from other nations, reports from the Federal Reserve Bank, and tables from the U.S. Department of Agriculture appeared to be ways of pushing the question back to the proxy without seeking assistance from other local DRSPs.

\section{Question 6}

Hi! What do the colors on the lifeguard flags mean?

At the request of the Florida Legislature, the Florida Department of Environmental Protection adopted the beach warning flag system. The Florida Department of Environmental Protection worked with the Florida Beach Patrol Chiefs Association, the United States Lifesaving Association, and the International Lifesaving Association to develop a uniform warning flag program for use by Florida's beachfront communities. The beach warning flag system recommended the following colors:

- Green: Low hazard, calm conditions

- Yellow: Medium hazard, moderate surf and/or currents

- Red: High hazard, rough conditions, strong surf and/or currents

- Red over Red: Water closed to the public

- Purple: Marine pests present

Florida was the first state in the nation to adapt the International Lifesaving Federation's beach flag system officially. 


\section{Researcher's Observations - Small-Sized Institutions}

Six of the 10 small-sized institutions responded. None of the DRSPs mentioned the Florida Legislature's request that the Department of Environmental Protection adopt a beach warning flag system. Four DRSPs provided an accurate answer. One of the DRSPs failed to provide the proxy with complete source information. One of the proxies also suggested an article from First Coast News, ${ }^{70}$ which mentioned the new flag system in place. One proxy included an article from the News Herald ${ }^{71}$ however, the DRSP weakened the credibility of the response by including details from the Carolina Beach in North Carolina, which omitted two flags, and from the U.S. Lifesaving Association's International Standards for Beach Safety and Information Flags, ${ }^{72}$ which omitted one flag.

Three DRSPs mentioned the U.S. Ocean Safety ${ }^{73}$ Web site; however, the site omitted the red over red flag and the purple flag. One DRSP indicated the meaning for the flag colors in Panama City and other Florida beaches. The DRSP neglected to include source information and details on two flag colors.

Students' Comments - Small-Sized Institutions

- $\quad$ Students offered no comments for question 6.

\footnotetext{
${ }^{70} \mathrm{http}: / / \mathrm{www}$. firstcoastnews.com

${ }^{71} \mathrm{http}: / /$ www.newsherald.com

${ }^{72}$ http://www.usla.org/PublicInfo/library/FlagWarningStandardsILSFinal20FEB04.pdf

${ }^{73} \mathrm{http}: / /$ www.usoceansafety.com
} 


\section{Researcher's Observations - Medium-Sized Institutions}

Eight of the nine DRSPs responded. Three DRSPs provided correct information to the proxy. One DRSP referred the proxy to $M S N B C,{ }^{74}$ which accurately described the beach warning flags, and mentioned the standardization of flag colors in Florida. Another DRSP correctly identified the flag colors as posted by the Orlando Sentinel ${ }^{75}$ on May 28, 2004, and by WESH.com ${ }^{76}$ on May 28, 2004. The DRSP also referred the proxy to consult lifeguards for verification. A third DRSP referred the proxy to $W J H G,{ }^{77}$ a television station in Panama City, Florida, which accurately listed the new recommended colors. This DRSP's response was weakened by the inclusion of two commercial vacation sites. One site omitted two flags. The other site omitted two flags and provided a questionable description for a third flag.

Four DRSPs mentioned the U.S. Ocean Safety ${ }^{78}$ Web site; however, the site omitted the red over red flag and the purple flag. One of the four DRSPs mentioned local beaches using the purple flag to warn swimmers about marine life present in the water. This DRSP also reminded the proxy to consult the lifeguard about beach conditions. The final DRSP mentioned the meanings of the green, yellow, and red flags, made no mention of two flags, and failed to provide source information.

\footnotetext{
${ }^{74} \mathrm{http}: / / \mathrm{www} . \mathrm{msnbc} \cdot \mathrm{msn} . \mathrm{com}$

${ }^{75} \mathrm{http}: / / \mathrm{www}$.orlandosentinel.com

${ }^{76} \mathrm{http}: / /$ www.wesh.com

77 http://www.wjhg.com

${ }^{78} \mathrm{http}: / /$ www.usoceansafety.com
} 


\section{Students' Comments - Medium-Sized Institutions}

- "It took long for her to respond the student. I wouldn't try and ask for her help."

- "Great job"

\section{Researcher's Observations - Large-Sized Institutions}

Seven of the nine DRSPs responded. Six DRSPs made no mention of the Florida Legislature's request that the Department of Environmental Protection adopt a beach warning flag system. One DRSP offered the proxy an Israeli site identifying different flag colors, a recommendation to check with a lifeguard, and a link connecting the proxy to a May 17, 2004 article on the First Coast News ${ }^{79}$ Web site. The First Coast News article included accurate information, as well as a mention of the new flag system in place. Another DRSP referred the proxy to the Web site for Beach Conditions: The City of Delray Beach ${ }^{80}$ where the colors were listed accurately.

The remaining DRSPs directed the proxy to a county government Web site listing three flags, a 2001 lifeguard manual available at the local library, the U.S. Lifesaving Association ${ }^{81}$ Web site listing four flags, the Lifesaving Resources Inc. ${ }^{82}$ Web page, which did not appear to list any flags, the Galveston Beach Patrol ${ }^{83}$ Web site in Texas listing three flags, the U.S. Ocean Safety ${ }^{84}$ Web site that failed to mention two flags, and the American Red Cross.

\footnotetext{
${ }^{79} \mathrm{http}: / / \mathrm{www}$. firstcoastnews.com

${ }^{80} \mathrm{http}: / /$ mydelraybeach.com/Delray/ Departments/ Parks+and+Recreation/Beach+ Conditions.htm

${ }^{81} \mathrm{http}$ ://www.usla.org

$82 \mathrm{http}: / /$ www.lifesaving.com

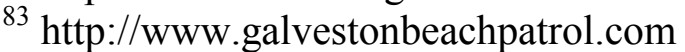

${ }^{84} \mathrm{http}: / / \mathrm{www}$. usoceansafety.com
} 


\section{Students' Comments - Large-Sized Institutions}

- "Good info but nothing to back it up"

Researcher's Observations - All Institutions

The Flag Conditions ${ }^{85}$ Web site emerged as the predominant resource consulted by 7 of the 21 DRSPs. This Web site included only three of the five flags recommended for Florida beaches. The question was relevant to the study because it was a current event in Florida several months before the proxy asked the question.

\section{Question 7}

Where can I find patient warnings for Celebrex? It's an anticonvulsant.

Celebrex ${ }^{\circledR}$ was not an anticonvulsant. It was an anti-inflammatory. Cerebyx ${ }^{\circledR}$ was an anticonvulsant. The DRSP might have misread the question, render a judgment rather than suggesting that the proxy seek a medical opinion from a credentialed expert, or direct the proxy to reputable medical databases, resources, or Web sites.

\section{Researcher's Observations - Small-Sized Institutions}

Four of the 10 DRSPs responded. Only one DRSP identified Celebrex ${ }^{\circledR}$ as an antiinflammatory, not an anticonvulsant. Two DRSPs recommended the PDR Family Guide to Prescription Drugs found in the Gale's Health and Wellness Resource Center database. The third DRSP referred the proxy to three Web sites including the U.S. Food

${ }^{85} \mathrm{http}: / /$ www.usoceansafety.com/safety/popup/flags.asp 
and Drug Administration (FDA) ${ }^{86}$ site, the Celebrex $\AA^{87}$ site, and an arthritis site that duplicated the FDA information. The fourth DRSP referred the proxy to the Celebrex ${ }^{\circledR}$ Web site. All of the recommended resources included patient warnings.

Students' Comments - Small-Sized Institutions

- "Not very friendly or helpful"

- "He or she did not answer my question and only told me where to get it from."

Researcher's Observations - Medium-Sized Institutions

Five of the nine DRSPs responded. None of the DRSPs questioned the proxy's identification of Celebrex ${ }^{\circledR}$ as an anticonvulsant. Four DRSPs used or suggested the $P D R$ Family Guide to Prescription Drugs in Gale's Health and Wellness Resource Center database. One of the DRSPs suggested the Physicians' Desk Reference if the patron was not a community college student.

Three DRSPs recommended nine Web sites. One site offered an overview of Celebrex ${ }^{\circledR}$ from the U.S. Food and Drug Administration's Center for Drug Evaluation and Research. Two sites provided warnings to the patient based on current information culled from the press, from the U.S. FDA, or from consumer experiences with the product. Two sites were online pharmacies providing both information and opportunities to purchase the pharmaceutical. One site was from the manufacturer of Celebrex ${ }^{\circledR}$ and it provided an overview of the medication and purchasing information. One site was a non-

\footnotetext{
${ }^{86} \mathrm{http}: / / w w w . f d a . g o v$

${ }^{87} \mathrm{http}: / /$ www.celebrex.com
} 
profit Web site directed at educating the consumer by offering a text-based and audio overview of Celebrex. The final site was an online drug resource tool.

\section{Students' Comments - Medium-Sized Institutions}

- "They weren't friendly"

- "Gave good information"

- “Wasn't that personable but when I need information, I just want that, I don't need 'chit chat."'

\section{Researcher's Observations - Large-Sized Institutions}

Seven of the nine DRSPs responded. Only one DRSP identified Celebrex ${ }^{\circledR}$ as an antiinflammatory, not an anticonvulsant. Two DRSPs were unclear as to the meaning of “patient warnings.” Another DRSP suggested an alternate term, "drug interactions," for patient warnings. Three DRSPs recommended Gale's online version of the Physicians' Desk Reference. Of these three DRSPs, two also recommended the paper version. The DRSP who identified Celebrex ${ }^{\circledR}$ as an anti-inflammatory also suggested the proxy check for information on both anti-inflammatory and anticonvulsant drugs, as well as a search of the Academic Search Premier and the Ovid databases.

Four DRSPs recommended Web sites from the U.S. Food and Drug Administration's Center for Drug Evaluation and Research, ${ }^{88}$ Pfizer's ${ }^{89}$ Web site for Celebrex ${ }^{\circledR}$, the U.S. National Library of Medicine ${ }^{90}$ Web site, and a commercial Web site for Celebrex ${ }^{\circledR}$. One

\footnotetext{
${ }^{88} \mathrm{http}: / / \mathrm{www} . f d a . g o v / \mathrm{cder}$

${ }^{89} \mathrm{http} / / /$ pfizer.com/pfizer/main.jsp

${ }^{90} \mathrm{http}$ ://www.nlm.nih.gov
} 
of the DRSPs e-mailed a document from the Pfizer site and identified the page numbers discussing the warnings.

One DRSP questioned the e-mail address of the proxy and opted to "dump" the request and report the message to another colleague. Since the DRSP copied the proxy on the e-mail, the proxy responded by acknowledging the need for the information, as well as the student's use of a myway email account. The DRSP then offered assistance through the telephone, a link to the library's Web address, and a recommendation to use the "Ask a Librarian" chat service for hours when the library was closed. The DRSP also "strongly" suggested that the proxy begin using the e-mail address assigned by the college. While the DRSP indicated a willingness to assist whenever "you need us," the proxy received no additional assistance with this e-mail. The proxy thanked the DRSP and the DRSP responded by referring the proxy to an in-class library instruction session scheduled by the instructor. After more than 66 hours, the DRSP also e-mailed the proxy an article from the PDR Family Guide to Prescription Drugs available from the Gale's Health and Wellness database. The DRSP provided an Internet address for the library's database, to the database itself, and a link to a help guide. The DRSP also provided limited instructions on accessing the library's databases, but did not suggest methods of searching.

\section{Students' Comments - Large-Sized Institutions}

- "She seemed very helpful although she didn't have interest in the topic"

- "To help a little more."

- "It was not friendly and you could give me detailed aid on the theme" 
- "Not friendly. Very short answer."

\section{Researcher's Observations - All Institutions}

Gale's Health and Wellness database, which included the PDR Family Guide to Prescription Drugs, emerged as the predominant resource recommended or consulted by 7 of the 16 DRSPs. The researcher designed the question to see how closely each DRSP read the request to identify the proxy's error. None of the DRSPs suggested the proxy seek the advice of a medical professional. None of the DRSPs provided information about obtaining a library barcode or password. None of the DRSPs provided information about logging in to the suggested databases. Only one of the DRSPs suggested instructions or methods for searching. Most of the sources mentioned included patient warnings.

\section{Question 8}

I'm writing an argumentative essay how do I find the material I need for my thesis? The topic is banning smoking in public places. I probably need 3 articles.

The researcher expected the DRSPs to direct the proxy to the online databases or ejournals and to provide assistance with a search strategy. The proxy asked for help retrieving three articles. The DRSP might have directed the proxy to LINCC for books, but the initial direction should have been to journals or magazines. A sample search strategy in the Academic Search Premier database might have been (ban* and smok* and public place* and (debat* or discus* or argu* or oppos*)). 


\section{Researcher's Observations - Small-Sized Institutions}

Four of the 10 small-sized institutions responded to the inquiry. The responses varied in terms of the level of detail suggested to the proxy. All of the DRSPs either suggested or sent articles from specific online periodical databases, i.e., Opposing Viewpoints, $C Q$ Researcher, Academic Search Premier, and FACTS.com. None of these DRSPs provided information about obtaining a library barcode or password. Two of the four DRSPs provided the Web page for accessing the library's databases and a third DRSP asked if the proxy knew how to access the databases. Two of the four DRSPs offered details on logging in to the databases. Three of the four DRSPs failed to suggest search strategies.

One of the four DRSPs asked if the question was for a Comp II class because those classes took a library tour. The DRSP invited the proxy to visit the campus or call for assistance. If time was an issue for the proxy, the DRSP recommended the use of the online Comp II tour. If the proxy had difficulty after the online tour, the DRSP urged the proxy to visit the campus since the instruction was difficult to explain through e-mail. If the proxy opted to use the databases without a site visit to the library, the DRSP provided only $\log$ in procedures.

\section{Students' Comments - Small-Sized Institutions}

- "Why not?"

Researcher's Observations - Medium-Sized Institutions

Seven of the nine DRSPs responded. The responses varied in terms of the level of detail suggested to the proxy. Six of the seven DRSPs either suggested or sent articles 
from specific online periodical databases, i.e., Opposing Viewpoints, OmniFile Full Text Mega, CQ Researcher, SIRS Researcher, Academic Search Premier, and Current Controversies. Six of the seven DRSPs failed to provide information about obtaining a library barcode or password. Four of the seven neglected to provide Web addresses for accessing the library's databases. Three of the seven DRSPs omitted details about logging in to the databases. Five of the seven DRSPs failed to suggest search strategies.

One of the DRSPs also referred the proxy to a non-profit Web site on the rights of nonsmokers ${ }^{91}$ and to the U.S. National Library of Medicine's anti-smoking campaigns. ${ }^{92}$ Although the DRSP said the proxy would find articles, the researcher found no articles. Another DRSP suggested circulating books, reference books, and Google in addition to the online databases. The DRSP reminded the proxy to contact the library, especially if the proxy needed help in citing sources. A third DRSP recommended the use of the library's online library instruction tutorial and the staff at the reference desk. A fourth DRSP referred the proxy to a library pathfinder and to LINCC. The library pathfinder included LINCC subject headings, reference titles, statistical sources, online databases, full-text online newspapers, and Internet sites.

\section{Students' Comments - Medium-Sized Institutions}

- "Friendly. A lot of help."

- "They were friendly, outgoing and very helpful."

- "Didn’t really answer the question."

\footnotetext{
91 http://www.no-smoke.org

${ }^{92}$ http://www.nlm.nih.gov/exhibition/visualculture/antismoking.html
} 
Researcher's Observations - Large-Sized Institutions

Seven of the nine DRSPs responded. One DRSP recommended database selection techniques while all seven DRSPs suggested databases or provided information about accessing the library's databases. Two DRSPs offered login assistance. Five DRSPs provided barcode or password details. Five DRSPs suggested search strategies. The databases suggested by the DRSPs included the Academic Search Premier, Opposing Viewpoints Reference Center, SIRS, Student Resources Center, OmniFile Full Text Mega, CQ Researcher, and FACTS.com. Other resources included the Internet, LINCC - the online public access catalog, online tutorials, library pathfinders, library visits, and telephone calls.

Two DRSPs reminded the proxy to contact the library for additional assistance. Another DRSP provided a list of potential article titles. One DRSP referred the proxy to LINCC and Google although the proxy asked for articles.

\section{Students' Comments - Large-Sized Institutions}

- "She was very helpful."

- "He or she was nice!!"

- "Good enough for me! :)"

\section{Researcher's Observations - All Institutions}

The Academic Search Premier database emerged as the predominant resource recommended by 9 of the 18 responding DRSPs. Seventeen DRSPs suggested the use of online databases to locate articles for the assignment. Twelve of the DRSPs neglected to 
include information about obtaining a library barcode or password. Six DRSPs neglected to provide details about accessing the databases. Ten DRSPs omitted information about logging in to the databases. Ten DRSPs failed to recommend a search strategy to the proxy. Only two DRSPs suggested Web sites or Google.com to locate additional information.

\section{Question 9}

Hi there. I'm getting married in a few months. My boyfriend gave me a list of his relatives. I can't find the zip for Menlo Park Ter in NJ. He said it's near the Edison tower. Hope you can find it for me. Thanks for your help.

The zip code for Menlo Park Terrace was 08840. It was located in Woodbridge Township, Middlesex County, New Jersey. It was about 1.8 miles from the Edison Tower.

Based on the incorrect responses resulting from this question, the researcher sent an email to the contact listed on the U.S. Postal Service's ZIP Code Lookup ${ }^{93}$ Web page asking the USPS to add the ZIP code for Menlo Park Terrace. As of November 2, 2005, the ZIP code had not yet been added to the database.

Researcher's Observations - Small-Sized Institutions

Five of the 10 DRSPs responded. None of them provided an accurate response. All of the DRSPs indicated 08837 for Menlo Park in Edison. Two DRSPs indicated 08820 for Menlo Terrace in Edison, New Jersey. One of the DRSPs answered based on the

\footnotetext{
${ }^{93}$ http://zip4.usps.com/zip4/welcome.jsp
} 
proximity of the Edison Tower. Two DRSPs asked additional questions to narrow down the search. The USPS ZIP Code Lookup ${ }^{94}$ database indicated that Menlo Park Terrace was not found in the database and the message said, "The city you entered could not be recognized or found in Menlo Park Terrace NJ in our database. Please confirm the spelling of the city name and try again."

Students' Comments - Small-Sized Institutions

- "Info was not correct"

\section{Researcher's Observations - Medium-Sized Institutions}

Six of the nine DRSPs responded. One DRSP accurately provided the proxy with 08840 as the ZIP code for Menlo Park Terrace and cited the Rand McNally Commercial Atlas as the resource. One DRSP referred the proxy to the Post Office for additional assistance. Three DRSPs indicated 08837 for Menlo Park, New Jersey. Four DRSPs referred the proxy to the USPS ZIP Code Lookup ${ }^{95}$ database. A fifth DRSP indicated not being able to find Menlo Park Terrace in the database. One DRSP also provided a Web site for information about Menlo Park, New Jersey, not Menlo Park Terrace, New Jersey. The DRSP indicated an uncertainty with the name of the city, but did not solicit additional questions from the proxy.

Students' Comments - Medium-Sized Institutions

- "Not helpful"

\footnotetext{
94 http://zip4.usps.com/zip4/welcome.jsp

${ }^{95} \mathrm{http}: / /$ zip4.usps.com/zip4/welcome.jsp
} 
- "She made it sound like she knew what was going on. I didn't enjoy her at all."

- "They should have said "I don't know" instead of giving wrong answer."

Researcher's Observations - Large-Sized Institutions

Eight of the nine DRSPs responded. None of them provided an accurate response. One DRSP indicated difficulty in answering the question because of uncertainty with Menlo Park Terrace as a city or as a street. This DRSP provided the proxy with 08837 for Menlo Park, New Jersey and 07095 for the Menlo Park Terrace section of Woodbridge, New Jersey. Menlo Park Terrace is a section of Woodbridge Township, but some sections have individual ZIP codes. This DRSP correctly made the connection to the Menlo Park Terrace section of Woodbridge Township, but failed to locate the right zip code.

Four DRSPs indicated a ZIP code of 08837; however, one response was based on the 2003 National Five Digit ZIP Code and Post Office Directory, two responses were based on the USPS ZIP Code Lookup, ${ }^{96}$ and one response had no source information. One DRSP suggested a site visit to the local U.S. Post Office. Two additional DRSPs suggested the USPS ZIP Code Lookup ${ }^{97}$ database for further assistance; however, neither conducted a search to confirm the veracity of the answer. One more DRSP referred the proxy to the USPS ZIP Code Lookup ${ }^{98}$ Web page and indicated five separate ZIP codes for Edison, New Jersey. The DRSP suggested that the proxy visit the site and input the street address for the correct ZIP code. The DRSP also recognized the need for the proxy

\footnotetext{
96 http://zip4.usps.com/zip4/welcome.jsp

${ }^{97} \mathrm{http}: / /$ zip4.usps.com/zip4/welcome.jsp

${ }^{98} \mathrm{http}$ ://zip4.usps.com/zip4/welcome.jsp
} 
to find other ZIP codes for her wedding invitations and suggested this Web site as a resource.

One of the DRSPs invited the proxy to double-check the 08837 answer at the Melissa Data $^{99}$ Web page and at the Menlo Park areaConnect ${ }^{100}$ Web page. Melissa Data was a commercial source that provided contact products or services. The Menlo Park areaConnect Web page was a commercial site for Menlo Park, California.

\section{Students' Comments - Large-Sized Institutions}

- "Wrong answer and not helpful"

- "Her answer was wrong."

\section{Researcher's Observations - All Institutions}

The United States Postal Service's ZIP Code Lookup ${ }^{101}$ database emerged as the predominant resource recommended by 10 of the 19 responding DRSPs. The database did not show Menlo Park Ter as one of the cities indexed in the database, so the DRSP needed to utilize another resource to provide a correct response to the proxy. Only one DRSP answered this question accurately.

\section{Question 10}

Who can help me with a problem I am having with searching the library databases online. I login fine, get all the way through to Connect to Database, and get

\footnotetext{
${ }^{99} \mathrm{http}: / / \mathrm{www}$. melissadata.com

${ }^{100} \mathrm{http}: / /$ menlopark.areaconnect.com

${ }^{101} \mathrm{http}: / /$ zip4.usps.com/zip4/welcome.jsp
} 
"Authentication failed due to insufficient credentials." My card works fine on the library computers. Please help or direct me to who can assist me.

The proxy indicated success using the library barcode at the library itself, so other possible problems might have caused failure. The usual problems for "Authentication failed due to insufficient credentials" included:

- $\mathrm{AOL}$ as the student's Internet service provider

- Browser or browser version

- Firewall, and

- High security setting

\section{Researcher's Observations - Small-Sized Institutions}

Four of the 10 DRSPs responded. The proxy indicated no problem in using the databases on the library's computers; however, three DRSPs repeated the login steps the proxy had already taken at the library. They offered no possible solutions. One DRSP asked which database the proxy was attempting to access. One DRSP ascertained that the problem had to do with the computer. This same DRSP directed the proxy to seek assistance from the reference desk, but not a particular person. A third DRSP asked for additional information to troubleshoot the proxy's question.

\section{Students' Comments - Small-Sized Institutions}

- "No, because did not show enough concern for the problem. No suggestion or even ideas to fix the problem." 
- 'Seemed the employee did not care about the student's problems. Trying to fix a problem or ask a question that was not even asked this is a lack of motivation and a pitiful display of customer service."

\section{Researcher's Observations - Medium-Sized Institutions}

Six of the nine DRSPs responded. Two DRSPs recommended that the proxy contact the circulation department to speak with a particular individual and provided the contact's name and telephone number. A third DRSP admitted not knowing why the proxy received the message and directed the proxy to contact the Microcomputers Office. None of the three DRSPs offered possible solutions, nor did the third DRSP act as an intermediary between the proxy and the Microcomputers Office staff. Two DRSPs repeated the barcode and PIN instructions although the proxy indicated success using both.

The fourth DRSP identified the incompatibility between AOL and the EBSCO databases as a possibility, but did not tell the proxy how to access EBSCO databases with AOL as an Internet service provider. Instead, the DRSP suggested using databases that did not cause problems with AOL.

The fifth and sixth DRSPs suggested a firewall as a possible reason. The fifth DRSP recommended disabling the firewall and attempting to access the databases again. If the proxy still encountered problems, the DRSP asked the proxy to contact the reference desk or to send an e-mail. The DRSP provided a telephone number. The sixth DRSP also suggested updating the browser or checking the security level on the computer. This 
DRSP provided steps to take. The DRSP recommended that the proxy contact the reference desk should the problems continue and provided a telephone number.

\section{Students' Comments - Medium-Sized Institutions}

- Students offered no comments for question 10.

Researcher's Observations - Large-Sized Institutions

Six of the nine DRSPs responded. Only one DRSP identified correctly a variety of potential problems, including AOL's incompatibility with some of the databases, firewall or security problems, browser options, database access page, and other database access issues. A second DRSP referred the proxy to the library's Web site where a list of possible reasons and solutions appeared.

One of the four remaining DRSPs referred the question to a support staff member but offered no possible solutions for the proxy to attempt. Another DRSP suggested that the proxy search another database. If the proxy accessed the second database without difficulty, then the problem with the first database was technical and vendor-related. The DRSP requested that the proxy let the library know if problems still existed. The DRSP offered no other possible solutions. The third remaining DRSP recommended that the proxy attempt to login by following the same steps already taken. The DRSP provided the telephone number for the reference desk if difficulties continued, but did not identify a particular person. The fourth remaining DRSP suggested a hardware problem and urged the proxy to contact Tech Support. While the DRSP provided a telephone number, the 
proxy received no possible solution, nor did the DRSP secure the answer from Tech Support before responding to the proxy.

Students' Comments - Large-Sized Institutions

- Students offered no comments for question 10.

Researcher's Observations - All Institutions

Contacting the reference desk emerged as the predominant response by 5 of the 16 respondents. Responses varied indicating a lack of understanding about potential access problems. Very few DRSPs appeared knowledgeable about the possibilities for "authentication failure." Several pushed the question back to the proxy by recommending the same steps that the proxy indicated taking. Given the number of years that the Florida community college libraries have had access to the online databases, the likelihood of these problems being new was low.

\section{Question 11}

Hello, I need to find some data on highway violence in Florida. Where can I go? Thanks so much.

Florida statistics could have been found on Florida's State ${ }^{102}$ Web site, specifically, the Florida Highway Patrol ${ }^{103}$ and the Florida Department of Highway Safety and Motor Vehicles. ${ }^{104}$

102 http://myflorida.com

${ }^{103} \mathrm{http}: / /$ www.fhp.state.fl.us

${ }^{104}$ http://www.hsmv.state.fl.us 
Researcher's Observations - Small-Sized Institutions

Five of 10 DRSPs responded. One DRSP identified the Florida Highway Patrol Drug Interdiction Statistics 1993-2002, which contained a short section on highway violence from 2001-2002. Another DRSP referred the student to the Florida Department of Highway Safety's 1999 Aggressive Driver Study. The study covered statistics from 19881997. The third and fourth DRSPs suggested resources that did not include the statistics requested by the proxy's question. Sources mentioned included an online aggressive driver quiz, a newsletter from the Florida Association of State Troopers, the Florida Department of Highway Safety's Traffic Crash Statistics Report 2003, and Schultz and Hunt's 1998 version of Traffic Investigation and Enforcement.

The fifth DRSP referred the proxy to numerous resources, including the OmniFile Full Text Mega database search resulted in 4,000 hits using the recommended terms "road rage" or "road rage" and statistics; a Canadian study from the Journal of Studies on Alcohol; a Google search on "road rage," statistics, and site:.gov that retrieved more than 300 hits but the first two pages of sites did not deal with Florida; and testimony from an administrator within the National Highway Traffic Safety Administration from 1997, which included estimated statistics. This DRSP also sent Garamone’s (2001) article stating, "The National Highway Traffic Safety Administration here says they have no hard statistics for aggressive driving and road rage incidents, but anecdotally they believe it is getting worse" (p. 1). Furthermore, this DRSP also indicated that the Statistical Abstract of the United States did not yet publish these statistics and recommended that the proxy use projections or estimates from online newsletters or Web sites. 
Students' Comments - Small-Sized Institutions

- "Very good. Site they sent student to was great."

Researcher's Observations - Medium-Sized Institutions

Four of the nine DRSPs responded. One of the four DRSPs provided a detailed, thorough response. This DRSP referred the student to the State of Florida's ${ }^{105}$ Web portal. The DRSP suggested searching myflorida.com for "road rage," which resulted in more than 90 hits. One of the articles was the Florida Highway Patrol's ${ }^{106}$ Web page detailing an article from the Gainesville Sun $^{107}$ on aggressive driving. The article provided statistics for both 2002 and 2003, as well as the date that the Florida Highway Patrol could begin issuing tickets for aggressive driving offenses. The DRSP also gave the student a Web link to the American Institute for Public Safety, ${ }^{108}$ which listed road rage documents, including several from Florida resources. The DRSP also mentioned an August 1998 article from the Atlantic Monthly addressing the issue of road rage. Finally, the DRSP recommended the student check the NewsBank database for articles, including the one attached to the e-mail; however, the DRSP neglected to include the location of the database, login information, and search strategy suggestions.

The other three DRSPs provided insufficient assistance. One of these three DRSPs referred the student to an online almanac and to Google. The DRSP offered no instructions on searching the almanac, but the researcher did find a link to the Florida

\footnotetext{
${ }^{105} \mathrm{http} / /$ www.myflorida.com

$106 \mathrm{http}: / / \mathrm{www}$.fhp.state.fl.us

${ }^{107} \mathrm{http}: / / \mathrm{www}$.gainesville.com

$108 \mathrm{http}: / / \mathrm{www}$.aipsnews.com
} 
Department of Highway Safety and Motor Vehicles ${ }^{109}$ with some extensive navigating. The DRSP also suggested a Google search for " "florida highway violence statistics' with or without the quotation marks," resulting in zero and 130,000 hits respectively. The student needed to search without quotation marks to access a variety of state and federal Web sites, but the student would have needed some direction on selecting an appropriate Web site for statistics. The second of the four DRSPs referred the student to the Florida Department of Transportation's ${ }^{110}$ Web page, which had no information to answer the question other than a link to the Florida Highway Patrol Web page. The third DRSP referred the student to two newspaper databases, Custom Newspapers and NewsBank. The instructions provided vague instructions to access, login, and search these databases and the search results offered the student hundreds to thousands of hits since the DRSP did not suggest using terms like Florida or statistics to limit the results.

Students' Comments - Medium-Sized Institutions

- "Great! Very helpful, incredible explanation!"

- "Awesome! Included multiple responses and areas for student to go back to! Was really good help!”

- "Explanation is hard to understand and shows lack of interest."

Researcher's Observations - Large-Sized Institutions

Five of the nine DRSPs responded. One of the five DRSPs suggested a variety of sources, i.e., traffic fatalities from the 2002 Florida Statistical Abstract, the 2003

\footnotetext{
${ }^{109}$ http://www.hsmv.state.fl.us

${ }^{110} \mathrm{http}: / /$ www.dot.state.fl.us
} 
Statistical Abstract of the United States, and Congressional Quarterly's 2000 State Fact Finder. The DRSP also listed four Web sites from various Florida State and U.S. agencies. The Florida Highway Patrol ${ }^{111}$ Web site listed activities in which the Patrol is involved. The U.S. Department of Transportation, Federal Highway Transportation's Highway Statistics $2000^{112}$ listed road details, use, fatalities, and more. The Florida Department of Highway Safety and Motor Vehicles' Agency Strategic Plan, ${ }^{113}$ published in 1999, included some highway violence statistics from 1993 through 1998. The Florida Highway Patrol's Drug Interdiction Statistics $2002^{114}$ included a section on highway violence.

One DRSP referred the student to numerous resources on road rage, i.e., three books from LINCC dated 1999 to 2000; assorted databases (Custom Newspapers, Academic Search Premier, InfoTrac, and the Student Resource Center) resulting in countless items dealing with the O. J. Simpson road rage case; and NetLibrary eBooks, which offered zero results for road rage or highway violence. Two other DRSPs also suggested using databases, i.e., Florida newspaper databases for the term road rage resulting in more than 150 articles within the past five years and Academic Search Premier database for "highway violence florida" resulting in zero hits.

Two DRSPs referred the proxy to the Florida Department of Highway Safety's Traffic Crash Statistics Report 2003; however, the data did not include statistics on highway violence. One of these two DRSPs asked if the student meant road rage. One DRSP referred the student to Google suggesting the search "highway violence florida." This

\footnotetext{
${ }^{111} \mathrm{http}: / / \mathrm{www}$. fhp.state.fl.us

$112 \mathrm{http}: / /$ www.fhwa.dot.gov/ohim/hs00/index.htm

$113 \mathrm{http}: / /$ www.hsmv.state.fl.us/reports/strategy.pdf

${ }^{114} \mathrm{http}: / / \mathrm{www}$. fhp.state.fl.us/html/stats.html
} 
search resulted in more than 180,000 hits; however, the Google search produced some key Florida resources at the top of the list.

Students' Comments - Large-Sized Institutions

- "Library employee was very friendly. Even took time to look up more information."

Researcher's Observations - All Institutions

The Florida Department of Highway Safety's Traffic Crash Statistics Report 2003 and Google emerged as the predominant resources recommended respectively by 3 of the 14 responding DRSPs. For the most part, the DRSPs neglected to consider state agencies for their answers. The DRSPs recommended 27 other resources for this question although they neglected to recommend Florida's government agencies as the question warranted.

\section{Question 12}

I am having a hard time finding some books on my topic. I checked LINCC and I am sure I don't know what I am doing, can you help? I want to find some books about selfimage and how it starts with Barbie. Does the library have books like this on the shelf? What do I do if you don't have any books? I need at least two books for my bibliography. Thanks.

Two books emerged specifically mentioning Barbie in the title; however, a search of women and (body image or self-esteem) may have resulted in related titles.

- Body Burden, Living in the Shadow of Barbie by Stacey Handler 
- Adios, Barbie: Young Women Write about Body Image and Identity by Ophira Edut (editor)

Researcher's Observations - Small-Sized Institutions

Six of the 10 DRSPs responded. Four DRSPs recommended one or more book titles. One DRSP placed the books on hold for the student and took the time to quote a few of the Barbie related passages to the student. One of the DRSPs recommended interlibrary loan and the potential to have the books delivered from other Florida libraries within two to three days. Three DRSPs indicated the interlibrary loan wait for titles not owned by the college's library to be about one week. One of the four DRSPs mentioned interlibrary loan specifically to enable the student to borrow a book written by a university faculty member. One of the four DRSPs neglected to tell the student how to go about retrieving the book from the shelf or if the book would be on a hold shelf.

Knowing that the proxy had already searched LINCC, two of the four DRSPS suggested various search terms, i.e., self-image, self-perception, and Barbie dolls, to further assist the proxy. Another of the four indicated that the student should use the LINCC subject headings from one book to locate other potential titles. Two DRSPs also recommended that the student conduct an "ALL COLLEGES" search to expand the number of results in LINCC. An "ALL COLLEGES" LINCC search enabled the user to search the 28 community college online catalogs at one time.

In addition to LINCC, one DRSP suggested the use of the library's online periodical databases using the same terms. Two DRSPs recommended the use of NetLibrary, but 
did not provide the student information about accessing, logging in, or searching this database. NetLibrary had some titles useful to the student.

Two DRSPs took five and six days respectively to respond to the inquiry. One said, "We have all been at workshops." One DRSP asked about the student's affiliation before the staff offered assistance.

Students' Comments - Small-Sized Institutions

- "Great job"

\section{Researcher's Observations - Medium-Sized Institutions}

Six of the nine DRSPs responded. Four DRSPs suggested anywhere from one to four titles by searching LINCC for topics such as Barbie and gender, self esteem and children, self, self help, self-image, body image, personal beauty, fashion, self esteem and social aspects, and pop culture. One DRSP reminded the proxy about the need to bring a student ID to borrow books. One DRSP urged the proxy to visit the library to consult the books since "I don't know that any of these will actually mention Barbie, but they may." This DRSP sent a second message stating that the student's library card also enabled access to the public library and to the local university collection. None of the four DRSPs mentioned interlibrary loan as an option for books specific to Barbie, nor did they suggest a LINCC search for "ALL COLLEGES."

Two of the four DRSPs also suggested the use of eBooks for other books that mention Barbie. One provided basic instructions for searching NetLibrary. One also recommended the use of FindArticles, Opposing Viewpoints, Academic Search Premier, and OmniFile 
Full Text Mega databases. The Web addresses, login information, and suggested search strategies were all provided to the student.

One DRSP recommended a Google search on Barbie doll image, which resulted in more than 290,000 hits with the majority of results within the first few pages focused on pictures or encyclopedia articles. One DRSP asked the proxy about his or her affiliation with the institution. The proxy indicated that she was a student and she had taken a psychology class the previous semester with a specific faculty member. The DRSP never responded to the student.

Students' Comments - Medium-Sized Institutions

- Students offered no comments for question 12.

\section{Researcher's Observations - Large-Sized Institutions}

Seven of the nine DRSPs responded. Four DRSPs suggested anywhere from two to six titles dealing with Barbie and self-image. Titles resulting from the suggested search times included Adiós Barbie: Young Women Write About Body Image, Identity and Body Outlaws and The Secret Lives of Girls. Two DRSPs placed the books on hold for the proxy. Two DRSPs provided several search terms and the search strategy for the proxy to check in the LINCC catalog. Another DRSP shared techniques for using both the index in the back of the book and relating broader concepts to specific ones. One DRSP did not suggest subject headings to the proxy. Five DRSPs did not suggest searching LINCC, the online public access catalog, for "ALL COLLEGES." 
Two DRSPs referred the proxy to librarians when on campus to locate other titles in LINCC. One of these two DRSPs also suggested visiting a librarian to learn how to access the online databases for journal and magazine articles. One DRSP suggested that the proxy browse the stacks in the same call number area as the suggested titles. Five DRSPs did not mention interlibrary loan as an option for books specific to Barbie and one DRSP did. Three DRSPs suggested that the proxy check the local public library for titles.

Three DRSPs recommended that the proxy consult the online databases to supplement the use of books. Suggested databases included Academic Search Premier, Student Resource Center, InfoTrac, Custom Newspapers, and NetLibrary. The NetLibrary titles that included information about Barbie related to gender, image, or popular culture. The proxy received instructions on accessing NetLibrary from only one DRSP. This DRSP provided access and login information; however, suggested search strategies were not suggested.

Students' Comments - Large-Sized Institutions

- "Wow, this is wonderful. Finally, a librarian that responds in time and with a great answer."

- "Good answer"

- "She was very polite and helpful" 
Researcher's Observations - All Institutions

LINCC, the online public access catalog, emerged as the predominant source recommended by 10 of the 19 responding DRSPs. Most of the DRSPs forgot to recommend the availability of interlibrary loan, reciprocal borrowing among community college and university libraries, and the ability to search LINCC for "ALL COLLEGES."

\section{Question 13}

Help, I am writing an MLA paper. I need to set up a reference. It looks really weird. Okay, the book has a bunch of chapters written by different people. So far, I have thisEditor: William Schutte

Title: Twentieth century interpretations of a portrait of the artist as a young man Publisher: Prentice Hall

Date: 1968

Author of the chapter: Lee T. Lemon

Chapter name: A portrait of the artist as a young man: motif as motivation and structure It starts on page 40 and ends on page 52 . This is where it gets weird. Way at the bottom of page 41, it has different info. I'm gonna type it just like the book says.

"A Portrait of the Artist as a Young Man: Motif as Motivation and Structure" by Lee T.

Lemon. From Modern Fiction Studies, XII (Winter 1966-1967), 439-450. Copyright 1965 by Purdue Research Foundation, Lafayette, Indiana.

The recommended responses included one of the following citations:

Lemon, Lee T. "A Portrait of the Artist as a Young Man: Motif as Motivation and Structure." Modern Fiction Studies 12 (1966-67): 439-50. Rpt. in Twentieth 
Century Interpretations of A Portrait of the Artist as a Young Man. Ed. William Schutte. Englewood Cliffs, NJ: Prentice Hall, 1968. 41-52.

OR

Lemon, Lee T. "A Portrait of the Artist as a Young Man: Motif as Motivation and Structure." Modern Fiction Studies 12 (1966-67): 439-50. Rpt. in Twentieth Century Interpretations of "A Portrait of the Artist as a Young Man." Ed.

William Schutte. Englewood Cliffs, NJ: Prentice Hall, 1968. 41-52.

Researcher's Observations- Small-Sized Institutions

Five of the 10 DRSPs responded, but only three mentioned using the MLA Handbook, $6^{\text {th }}$ edition. One DRSP correctly identified the section in the MLA Handbook, but presented the publication information incorrectly. The second DRSP referred the proxy to the Handbook, but not the section, and to two Web sites. The Web sites did not address the proxy's question. The third DRSP referred the proxy to the Handbook and recommended two anthology examples; however, the examples did not address the proxy's question. The proxy was not given information on locating the MLA Handbook at the library.

The fourth DRSP suggested an incorrect format lacking both the original source information and the reprint information. The fifth DRSP suggested that the proxy use the Citation Machine -- The Landmark Project ${ }^{115}$ Web site. The generator created an electronic or traditional citation based upon the information entered by the user. If the user failed to capitalize necessary parts of the citation or spells words incorrectly, the

$115 \mathrm{http}: / /$ citationmachine.net 
citator did not flag the error. The citator did not allow the proxy to input an anthology entry for a journal article or to indicate a reprint. Only one DRSP suggested that the proxy consult his or her instructor.

Students' Comments - Small-Sized Institutions

- Students offered no comments for question 13.

Researcher's Observations- Medium-Sized Institutions

Seven of the nine DRSPs responded. Two DRSPs referred the proxy to the $M L A$ Handbook. One DRSP referred the proxy to the correct page in the current $M L A$ Handbook. The other DRSP referenced section 5.6.8 of the MLA Handbook; however, the correct section is 5.6.7. This DRSP also provided the proxy with a sample citation. Although the entry was partly accurate, enough errors appeared to warrant the citation problematic for the proxy, i.e., underlining the title of the novel, publication date, Roman numerals, and punctuation marks.

Two DRSPs suggested using online citation guides located on their respective library's Web site. One guide did not provide an anthology entry and it referenced an older edition of the MLA Handbook. The guide also suggested a Web site; however, the site did not provide an example a journal article reprint. One guide addressed citing uncommon sources. The guide addressed journal articles reprinted in anthologies; however, the citation examples had formatting and punctuation errors. 
Two DRSPs recommended Diana Hacker's ${ }^{116}$ Web site to check the "Work in an Anthology" section although details on citing a journal reprint were not evident. One of the DRSPs offered the proxy additional assistance by telephone or by e-mail. One DRSP also referred the proxy to the aforementioned Citation Machine -- The Landmark Project ${ }^{117}$ Web site and to Capital Community College's Library ${ }^{118}$ Web site where the suggested format was for a magazine article.

One DRSP provided the proxy with a sample citation that was partly accurate. Enough errors appeared in the entry to warrant the citation problematic for the proxy, i.e., use of punctuation and quotation marks, spacing, publication dates, use of Roman numerals. Another DRSP stated that the proxy did not have to indicate the anthology information in the citation and provided what appeared to be an original journal citation. Even within the original citation, the DRSP had both formatting and punctuation errors with underlining, spacing, dating, and capitalization.

\section{Students' Comments - Medium-Sized Institutions}

- Students offered no comments for question 13.

\section{Researcher's Observations- Large-Sized Institutions}

Seven of the nine DRSPs responded. Two DRSPs mentioned use of the MLA Handbook directly. One of the two DRSPs referred the proxy to section 4.6.7 of the $M L A$ Handbook; however, the correct section was 5.6.7. The DRSP provided the proxy with a

\footnotetext{
$116 \mathrm{http}: / / \mathrm{www}$.dianahacker.com

$117 \mathrm{http}: / /$ citationmachine.net

${ }^{118} \mathrm{http}$ ///www.ccc.commnet.edu/library/index.htm
} 
sample citation. Although the entry was partly accurate, enough errors appeared to warrant the citation problematic for the proxy. The DRSP indicated, "I don't think you need to be concerned with the fact that it was also published elsewhere in a periodical since you are getting if from the book source." The DRSP omitted formatting and punctuation instructions. The second DRSP correctly referred the proxy to the $M L A$ Handbook, $6^{\text {th }}$ edition, page 160 . The DRSP also provided the proxy with contact information so that the proxy and the DRSP could work together to set up a suitable citation.

One DRSP referred the proxy to a guide on the library's Web site. This DRSP provided the proxy with an example of a journal reprint in an anthology, but neglected to include punctuation and formatting instructions. The example used was also for a short story, not a novel. The DRSPs also recommended several Web sites. The Purdue Writing $L a b^{119}$ Web site was listed on the aforementioned library's Web site. The Writing Lab showed an essay in an anthology, but not a journal reprint. Also listed on this same Web site and showing only online citations was the Bedford Martin Guide ${ }^{120}$ Web site. Another DRSP referred the proxy to the MLA documentation guide on the St. Cloud State University's ${ }^{121}$ Web site. The guide did not offer an example for an original journal reprinted in a book. The guide did refer the proxy to the MLA Handbook; however, the DRSP did not share information about locating the needed examples.

One of the DRSPs contacted an MLA expert at his or her college. The expert indicated, "Just site [sic] it as a signed article in an edited book. Don't need to site [sic]

\footnotetext{
119 http://owl.english.purdue.edu

${ }^{120} \mathrm{http} / / /$ bcs.bedfordstmartins.com/bedguide/default.asp?uid $=0 \& \mathrm{rau}=0 \#$

${ }^{121} \mathrm{http}$ ://leo.stcloudstate.edu/research/mlaparen.html
} 
the original journal publication info." The DRSP and institutional MLA expert neglected to cite the journal article as a reprint in an anthology, nor did they show the proxy how to underline the title of the novel both in the name of the article title and in the name of the source. Another DRSP indicated that the book itself needed to be cited, but the chapter information did not need to be included in the citation. Yet another DRSP sent the proxy the citation setup. Although the entry was partly accurate, enough errors appeared to warrant the citation problematic for the proxy, i.e., formatting and punctuation.

Students' Comments - Large-Sized Institutions

- "Bad. Re-read the question."

Researcher's Observations- All Institutions

The MLA Handbook emerged as the predominant resource recommended by 8 of the 19 respondents. The DRSPs also relied on library-created guides or citation generators; however, many of these tools showed problems with formatting.

\section{Question 14}

Hi, I have to do a speech in class on the "Hershey" company. I need to know the date of incorporation, the main lines of business, last year's sales, profits, and federal income tax paid. Where can I go?

The answer was the corporate Web site at http://www.hersheys.com. 
Researcher's Observations - Small-Sized Institutions

Six of the 10 DRSPs responded. Five DRSPs suggested online databases, but neglected to provide the proxy with information on accessing the databases, using a password, and methods for searching the resource. One DRSP failed to inquire about the patron's status and instead directed the patron to unspecified resources for each category of user (community college, university, or public patrons). The DRSP did not provide instructions to the proxy because the proxy's status was never ascertained. Two DRSPs also sent documents about Hershey; however, the documents did not provide the requested financial information. The documents supplemented other resources. One DRSP reminded the proxy to visit the library or call for additional help; however, the DRSP neglected to provide a telephone number.

\section{Students' Comments - Small-Sized Institutions}

- Students offered no comments for question 14 .

\section{Researcher's Observations - Medium-Sized Institutions}

Six DRSPs responded. Four DRSPs referred the proxy to the Hershey ${ }^{122}$ Web site. Three DRSPs offered instructions for navigating the page to locate the information requested by the proxy. The fifth DRSP referred the proxy to the local county public library to use the collection of annual reports since the library had no resources to answer the proxy's questions. The sixth DRSP sent the proxy a canned response to the reference inquiry directing the proxy to available options. The canned response directed the proxy

${ }^{122} \mathrm{http} / / / \mathrm{www} \cdot h$ hersheys.com 
to a set of sources in particular disciplines; however, the Web site provided to the proxy was incorrect. If the proxy was able to access the correct Web site, the DRSP had not provided guidance as to which of the 60 or more links would most likely assist the proxy in answering the reference question.

One of the six DRSPs referred the proxy to two business reference books, Hoover's Handbook of American Business for 2004 and Mergent's Handbook of Common Stocks for summer 2004. As well as referring the proxy to these sources, the DRSP provided some of the answers the proxy requested. Two DRSPs also mentioned several financial Web sites that included information answering the proxy's questions. One DRSP mentioned several reference books that included financial information; however, using these sources required a site visit. One DRSP urged the proxy to consult a DRSP for additional assistance.

\section{Students' Comments - Medium-Sized Institutions}

- "The answer is short but accurate."

- “Didn't answer the question. Didn't sign their name - I don't know who answered the email and could have been Joe Blow from the sheet [sic]."

- "Good info."

Researcher's Observations - Large-Sized Institutions

Five of the nine DRSPs responded. Five DRSPs referred the proxy to the Hershey Web site. Only three DRSPs directed the proxy to specific parts of the site, including the financial and annual report sections. 
One DRSP also suggested a Google search for "Hershey date of incorporation" and for "Hershey profits" to locate results; however, the searches resulted in more than 6,500 results and more than 36,000 results respectively. The DRSP did not provide guidance as to which Web sites might be most useful for the proxy. Four DRSPs suggested various financial Web sites, including Hoover's Online ${ }^{123}$ Web site, Yahoo's Quicken ${ }^{124}$ Web site, and the Corporate Investor Relations ${ }^{125}$ Web site. The Hoover's Web site was proprietary; however, the overview referred the user to the Hershey's corporate Web site and it included SEC filings. The Corporate Investor Web site referred the proxy to the investor relations portion of the corporate Web site.

Two DRSPs suggested EBSCO’s Business Source Elite database, but only the Web address was provided. A subsequent search led to more than 2,200 articles, as well as a company profile. The company profile, prepared by Datamonitor, provided a 30-page overview of the company, including the corporate Web site, which offered the information sought by the proxy. A third DRSP also suggested the Academic Search Premier and Business Source databases, but sent the proxy password information, the online location, and a suggested search strategy. One DRSP suggested the proxy consult Lexis Nexis' business resources. The DRSP directed the proxy to the Web address, reminded the proxy to use a library barcode and password, but did not share any searching hints.

One of the DRSPs recommended the International Directory of Company Histories, located in the library's reference collection that necessitated a site visit to campus. The

123 http://www.hoovers.com

$124 \mathrm{http}: / /$ finance.yahoo.com

${ }^{125}$ http://www.corporate-ir.net 
DRSP also referred the proxy to Hoover's Handbook of American Business for 2003, Value Line, and Company Profiles for Students. The DRSP did not check the local library or the college library for holdings information, nor was information given regarding the local library or the college library. The DRSP took the time to quote various portions of these resources to the proxy and included page numbers for traditional resources. The DRSP also offered to fax the appropriate pages to the proxy. The DRSP shared some facts about Hershey with the proxy, but did not indicate the source used for the facts.

Two DRSPs suggested using the local public library. One recommended its use for hours when the community college library was closed. The other DRSP referred the proxy to the local public library's business department since it "has all of this information and more."

\section{Students' Comments - Large-Sized Institutions}

- "He gave the student a lot of information."

- "The answer is a little short but it will help a lot."

\section{Researcher's Observations - All Institutions}

The Hershey ${ }^{126}$ Web site emerged as the predominant source used by 11 of the 19 responding DRSPs. EBSCO's Business Source Elite database and Hoover's Online ${ }^{127}$ were each recommended by five DRSPs.

\footnotetext{
${ }^{126} \mathrm{http}: / / \mathrm{www} \cdot$ hersheys.com

$127 \mathrm{http}: / / \mathrm{www} \cdot$ hoovers.com
} 


\section{Question 15}

Is cellulitis hereditary?

The DRSP might have confused cellulitis with cellulite, rendered a judgment rather than suggesting that the proxy seek a medical opinion from a credentialed expert, or directed the proxy to reputable medical databases, resources, or Web sites for preliminary reading.

\section{Researcher's Observations - Small-Sized Institutions}

Five of the 10 DRSPs responded. The DRSPs appeared uncertain about cellulitis being hereditary. One indicated misgivings, one indicated a non-hereditary status, and one identified a specific type of cellulitis. Three DRSPs sent the proxy an article(s) from Gale's Encyclopedia of Medicine. Two DRSPs sent the proxy a comprehensive article from the National Library of Medicine ${ }^{128}$ and the National Institute of Health's MedlinePlus ${ }^{129}$ and a third DRSP suggested the use of both Web sites. Two DRSPs sent the proxy article(s) from Gale's Health and Wellness Resource Center database and a third DRSP referred the proxy to the Health and Wellness Resource Center database. A fourth DRSP suggested that the proxy access other electronic databases and search for health titles. Three DRSPs did not provide the proxy with information regarding barcode access, the location of the database, access information, and searching strategies. None of the DRSPs suggested seeking the advice of a credentialed medical expert.

\footnotetext{
${ }^{128} \mathrm{http}: / /$ www.nlm.nih.gov

$129 \mathrm{http}: / /$ medlineplus.gov
} 
Students' Comments - Small-Sized Institutions

- Students offered no comments for question 15.

Researcher's Observations - Medium-Sized Institutions

Seven of the nine DRSPs responded. Three DRSPs made the determination about the condition being hereditary, but did not refer the proxy to his or her physician for a medical opinion. Two DRSPs indicated a lack of authorization in answering medical questions and that "perhaps your best source of information on this topic may be your family doctor." Four DRSPs did not provide access information, database location, and search strategies. One DRSP provided the Web address to access the databases and information about the barcode and PIN number, but neglected to suggest a search strategy.

Two DRSPs sent the proxy articles from the Health and Wellness Resource Center database and Gale's Encyclopedia of Medicine. One article was more than 10 years old and dealt with only one type of cellulitis. Two DRSPs referred the proxy to the library's Web site to locate articles and suggested an assortment of health and medical databases. One DRSP provided the proxy with a one-sentence definition from Stedman's medical dictionary and referred the proxy to the library's online databases and dictionaries. One DRSP provided the proxy with two sentences from Gale's Encyclopedia of Medicine.

One DRSP suggested four Web sites for further information. The Web sites included DoctorElite, ${ }^{130}$ the Merck Manual,${ }^{131}$ HealthDayNews, ${ }^{132}$ and Expedition Network

\footnotetext{
${ }^{130} \mathrm{http}: / / \mathrm{www}$. doctorelite.com/drelite/faq4.html

${ }^{131} \mathrm{http}: / /$ www.merck.com/mrkshared/mmanual/section10/chapter112/112.jsp

$132 \mathrm{http}: / / w w w . a l t r u . o r g / h e a l t h n e w s / h e a l t h d a y / 040721$ HD519892.htm
} 
Limited's Medical Encyclopedia. ${ }^{133}$ Another DRSP referred the proxy to a series of research guides; however, the referring page included 12 pages of references, each with 25 or more suggested links. Most of the DRSPs did not suggest seeking the advice of a credentialed medical expert.

\section{Students' Comments - Medium-Sized Institutions}

- "Gave a lot of information"

- "Seemed to provide sufficient answer"

- "Very simple"

- "The librarian took a really long time to reply to this e-mail. His response to Joseph's question was pretty vague and only sited [sic] one source.”

Researcher's Observations - Large-Sized Institutions

Four of the nine DRSPs responded. Only one DRSP directed the proxy to his or her physician for a definitive answer; however, that DRSP provided the proxy with a basic search strategy for a Google search in case the proxy wanted to do some preliminary research. A second DRSP directed the proxy to the National Library of Medicine ${ }^{134}$ Web site, but the DRSP determined that the condition was not hereditary. A third DRSP suggested the proxy try a Google search for "cellulitis hereditary." The Google search resulted in more than 11,000 hits with many associated with animals. The other resource suggested by the DRSP was the WebMD ${ }^{135}$ Web site, which resulted in 53 hits with the

$133 \mathrm{http}: / /$ enlmedical.com/article/000855.htm

$134 \mathrm{http}: / /$ www.nlm.nih.gov

${ }^{135} \mathrm{http}: / / \mathrm{www} . w e b m d . c o m$ 
first four addressing overview, treatment, symptoms, and home treatment. The fourth DRSP directed the proxy to information about cellulitis located at four Web sites, Kidshealth, ${ }^{136}$ Mayo Clinic, ${ }^{137}$ Merck, ${ }^{138}$ and the National Library of Medicine. The DRSP also sent an article from EBSCO's Academic Search Premier database written from a physician's perspective. Most of the DRSPS did not suggest seeking the advice of a credentialed medical expert.

\section{Students' Comments - Large-Sized Institutions}

- "Because it seem [sic] he/she didn't put to much time to help this student out."

Researcher's Observations - All Institutions

Gale's Health and Wellness Resource Center database emerged as the predominant source recommended by 7 of the 16 respondents. Four DRSPS each suggested MedlinePlus ${ }^{139}$ and Gale's Encyclopedia of Medicine. The lack of access information, database location, and search strategies appeared to be an ongoing problem with the DRSPs. Another concern arising from this question was the lack of authority that most DRSPs had in providing medical advice.

\section{Summary of Results}

Twenty-three of the 28 community college libraries participated in the interview portion of the study. The DRSPs indicated that their libraries utilized the expertise of 98

\footnotetext{
${ }^{136} \mathrm{http}: / /$ www.kidshealth.org

${ }^{137} \mathrm{http}: / / \mathrm{www} . \mathrm{mayoclinic} . \mathrm{com}$

${ }^{138} \mathrm{http}: / /$ www.merck.com/mmhe/sec18/ch211/ch211b.html

${ }^{139} \mathrm{http} / / /$ medlineplus.gov
} 
Master's level librarians and one staff member with a bachelor's degree. Their longevity of library experience ranged from first year employees to 37 years. Twenty of the 23 libraries utilized the statewide synchronous training for "Ask a Librarian," informal internal training, or formal internal training.

Eighteen of the 23 interviewees indicated that their libraries did not survey e-patrons to assess their satisfaction with digital reference. More than half did not monitor the service for quality although most of the quality checks were done informally. Seventeen of the 23 DRSPs indicated that their libraries had no written policies or procedures in place for asynchronous reference services. The DRSPs varied in the types of questions received and answered by their respective services, as well as the patron type served in the asynchronous environment.

The DRSPs agreed that library service was a student support creating a bridge to student success and asynchronous service extends the reference services available to DL students. Since the student asked for and received assistance, the DRSPs indicated that the approach, response, encouragement, and timeliness to the student were critical to developing an online relationship. The DRSPs intimated that few online encounters engaged students and they had difficulty in determining students' computer, Internet, or research skills unless they were observable or discussed by the students. They said that email reference provided them the opportunity to spend more time working with the student.

Twenty-eight of Florida's community college libraries received unobtrusively 14 questions asynchronously through e-mail. Of 392 possible responses sent to DRSPs, the proxy received 240 responses (61\%). The researcher scored $24 \%$ as accurate with source 
information, $4 \%$ as accurate without source information, $20 \%$ as partly accurate with source information, and $7 \%$ as partly accurate without source information. The students scored $48 \%$ as accurate with source information, $12 \%$ as accurate without source information, $17 \%$ as partly accurate with source information, and $9 \%$ as partly accurate without source information. In terms of the $t$-Test for Two Independent Samples for accuracy and satisfaction, the researcher and the student scores showed no significant difference for 12 of 14 questions. The response time ranged from 6 seconds to 20 days. The DRSPs answered 10\% percent in less than one hour, $17.5 \%$ in one to two hours, 61\% within 24 hours, $75 \%$ within 48 hours, 86\% within 72 hours, $90 \%$ within 96 hours, and the remainder took from 5-20 days to answer the question.

The researcher and the students also assessed the communication between the DRSP and the proxy to learn about the development of an online relationship between them. In terms of the $t$-Test for Two Independent Samples for communication, the researcher and the student scores showed no significant difference for 12 of 14 questions. The researcher and the students scored the DRSPs below $75 \%$ for the following communication traits: greeting the proxy, using the proxy's name, showing interest in the proxy's question, demonstrating signs of a reference interview, providing steps for the proxy to duplicate the DRSP's steps, making spelling errors, asking whether the question was answered, inviting the proxy to utilize the service again, and listing the DRSP's position or title at the library. The researcher indicated a willingness to return to the DRSP for assistance in only $62.5 \%$ of the cases and the students indicated a $58 \%$ return rate. 


\section{Chapter 5}

\section{Conclusions, Implications, Recommendations, and Summary}

\section{Conclusions}

The purpose of the study was to examine the adequacy of asynchronous e-mail reference services offered to DL students at Florida's 28 community college libraries and the contribution of library staff to the students' online learning community. To accomplish this objective, the researcher considered two sets of questions for analysis. The first set of questions addressed the adequacy and accuracy of virtual reference service. The second set of questions addressed the role of the DRSP in the student's online environment.

The researcher asked these questions. Do asynchronous library support services adequately address reference requests made digitally by students? Do librarians respond to students in a timely manner? Do they ask additional or follow-up questions to comprehend fully the student's research request? How do librarians respond to student inquiries? Do they provide students with accurate information? Do they cite the sources used to answer reference questions?

Do these services enhance the student's online learning community? Does the librarian initiate an open and inviting dialogue? Does the librarian attempt to personalize the experience for the student? Does the librarian invite the student to use the service again should additional questions arise? 
Do asynchronous library support services adequately address reference requests made digitally by students?

According to Whitlatch (2000), “A response rate of 70 to 80 percent is considered good" (p. 30). Posing as a student, the researcher directed the same 14 questions to each of the 28 community college's digital reference service centers or providers for a potential 392 inquiries. The DRSPs answered 240 inquiries for a $61 \%$ return rate. Only two libraries answered all 14 questions (see Figure 2).

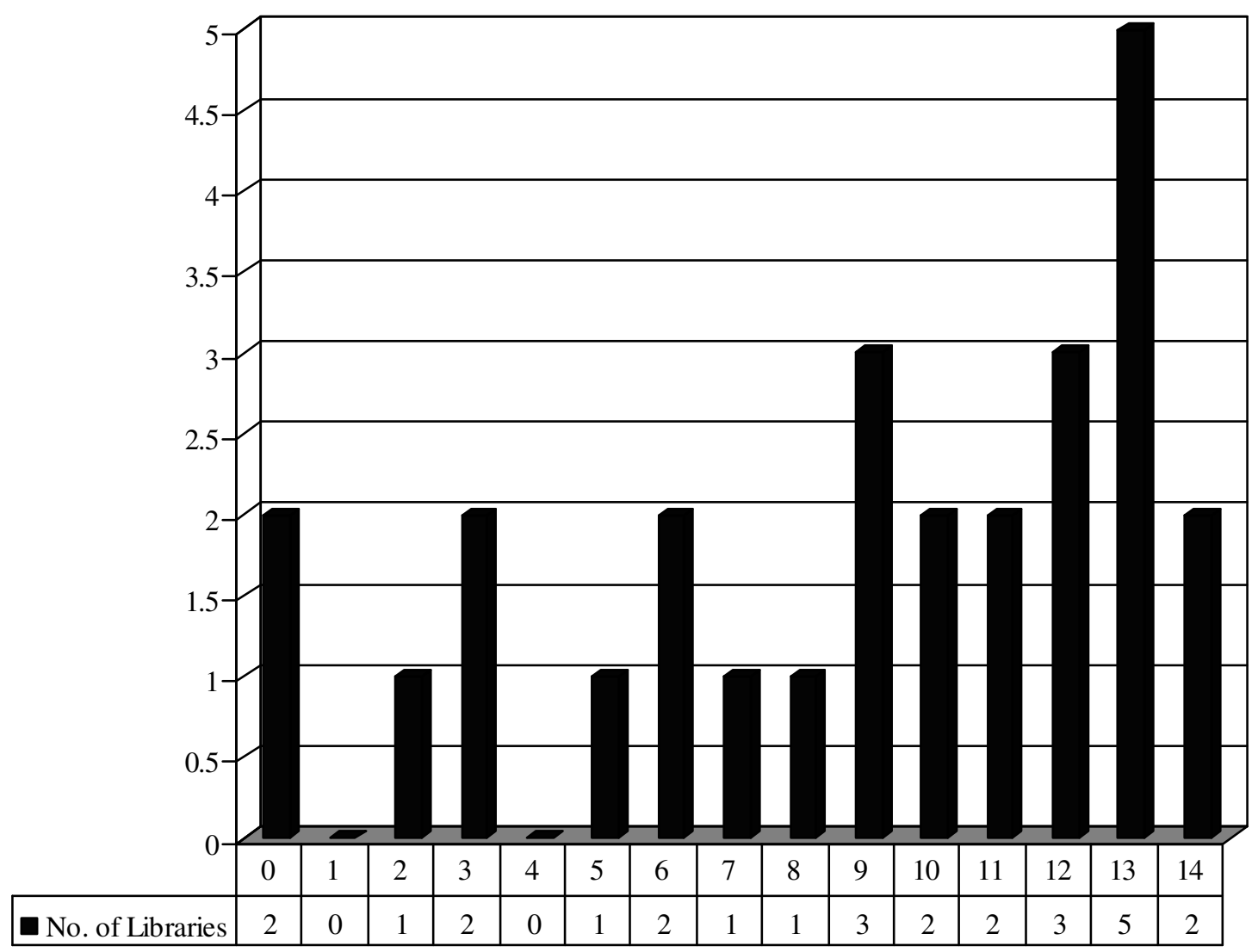

Figure 2. Number of questions answered by libraries.

The researcher interviewed the 23 DRSPs selected by their respective library administrators to gain an understanding of policies, procedures, beliefs about digital 
reference service, and attitudes about their role in the student's online learning environment. In order to reduce discovery of the study by the DRSPs, the interview questions were asked after data collection for the unobtrusive portion of the study. Of the 23 DRSPs interviewed by the researcher, they indicated that 99 individuals provided digital reference service, 98 of whom possessed graduate level degrees. These 99 DRSPs had less than 1 year to 37 years of service, with a mean of 16.5 years and a mode of 10 years of service, excluding two respondents who provided only a range of service dates. DRSPs obtained their digital reference training using a wide array of both formal and informal internal methods, as well as formal external training offered through Florida's “Ask a Librarian" program.

From these 23 interviews, 17 DRSPs acknowledged not having any policies for digital reference services in place and 18 DRSPs indicated that they did not survey their epatrons to assess their satisfaction with digital reference. Those libraries assessing their epatrons used the library's annual survey tool, Web site evaluation tool, course evaluations, library or service satisfaction tool, auto-questionnaires, or a survey tool sent immediately after the transaction. More than $50 \%$ of the DRSPs interviewed said they did not monitor digital reference service for quality. The question asked by the researcher prompted several to comment about their plans to conduct future assessments.

Of the 23 DRSPs interviewed, $61 \%$ indicated that they would assist anyone who asked questions with the exception of inappropriate questions; $30 \%$ would assist all patrons but might refer them to another library for additional assistance; and 13\% indicated they would assist all patrons, but might provide out-of-state patrons with college information only. From this same group, 78\% DRSPs indicated they did not restrict the types of 
questions asked by students; however, they referred questions to other college departments, college helpdesks, educational institutions, or public libraries when appropriate. The majority of the group discussed the policy as not being restrictive so much as the student's need being unsuitable for the online environment. They asked these students to contact the library by telephone, schedule a synchronous chat session, or explore an online tutorial. To assist students in the online environment, some libraries provided outreach to other campuses and to specific student groups, included handouts in college packets, and designed a usable Web site. One DRSP indicated that e-mail reference questions were a lower priority than other reference questions.

Seventeen of the 23 DRSPs interviewed received database access, searching, and troubleshooting inquiries (see Table 12). Inquiries about circulation services, such as reserve, due dates, renewals, and fines, were asked of $57 \%$ of the respondents. Of the group, $44 \%$ received research or reference questions and $44 \%$ fielded barcode, password, or library card questions. Public service questions about interlibrary loan, library collections, library instruction, and library handouts or questions from vendors were received by $31 \%$ of the respondents. Twenty-six percent of the respondents identified OPAC questions or searches as typical. Software, hardware, and Internet service provider questions were often received by $22 \%$ of the group. Informational requests about the college and the library's policies, procedures, and hours were received respectively by $17 \%$ of the group. Citation assistance and journal assistance were each identified by $13 \%$ of the group. Nine percent of all respondents typically received directional questions. Ready reference and referral questions were each received by $4 \%$ of the digital reference providers. The DRSPs answered all but homework, medical advice, legal advice, 
extensive genealogical research, inappropriate pornographic requests, and out of state research requests (see Table 12).

Table 12. Comparison of Questions Received/Deemed Appropriate by DRSPs

\begin{tabular}{lcc}
\hline \multicolumn{1}{c}{ Category } & Received & Appropriate \\
\hline Database & $73 \%$ & $26 \%$ \\
Circulation & $57 \%$ & $26 \%$ \\
Reference and Research & $44 \%$ & $13 \%$ \\
Barcode & $44 \%$ & $26 \%$ \\
Public Service & $31 \%$ & $17 \%$ \\
OPAC & $26 \%$ & $9 \%$ \\
Computer & $22 \%$ & $4 \%$ \\
Info Requests & $17 \%$ & $17 \%$ \\
Policies, Procedures, and Hours & $17 \%$ & $26 \%$ \\
Citations and Journals & $13 \%$ & $4 \%$ \\
Directional & $9 \%$ & $26 \%$ \\
Ready Reference and Referrals & $4 \%$ & $48 \%$ \\
\hline
\end{tabular}

The difference between those questions deemed appropriate by the DRSPs and those questions received by the libraries varied significantly. Since 17 (or 73\%) of the DRSPs acknowledged not having any policies for digital reference services in place, the types of questions received should determine policy rather than what the DRSPs deemed appropriate. Short or ready reference questions were deemed appropriate by $48 \%$ of the group. Database or online access questions were found to be reasonable by $26 \%$. Library hours, library services, password, or policy questions were regarded as fitting by $26 \%$ of the group. Assistance in locating additional, subject oriented, or specific class resources 
were determined to be suitable questions by $17 \%$ of the group. Research or searching type questions were found suitable by $13 \%$ of the group. Database or research recommendations or referrals were determined appropriate by $13 \%$ of the group. Collection or OPAC questions were deemed appropriate by $9 \%$ of the group. Citation assistance was identified by $4 \%$ of the group. Questions that concerned themselves with tutoring a student or with technological questions were determined inappropriate by $4 \%$ of the group. Library orientation requests were identified by $4 \%$ of the group as appropriate to e-mail reference service (see Table 12).

Do they provide students with accurate information? Do they cite the sources used to answer reference questions?

As suggested by Hernon and McClure (1987b), each library owned, subscribed, or had access to resources that enabled a successful response to the 14 inquiries. Hernon and McClure's 1986 study found that librarians typically answer 55\% of questions asked correctly (p. 41). The researcher determined an accurate response with or without source information in $28 \%$ of the responses and a partly accurate response with or without source information in $27 \%$ of the responses. The student assessors determined an accurate response with or without source information in $60 \%$ of the responses and a partly accurate response with or without source information in $26 \%$ of the responses. The researcher indicated source name and source information provided in $71 \%$ of the responses while the student assessors indicated 68\%. Pomerantz, Luo, and McClure (in press) learned from their 2004 unobtrusive study of virtual reference that librarians often 
sent answers or resources to patrons without "any explanation of the authority or provenance of those resources or why the librarian selected them" (p. 18).

In terms of accuracy and satisfaction, there was no significant difference between the researcher's averaged scores and the students' averaged scores in 12 of the 14 questions. Of 14 questions, the researcher found no excellent responses; two responses that were average, accurate, and effective; one response that needed some improvement in the accuracy category, but showed redeeming features; six responses that showed the need for improvement in the accuracy category; and five responses that showed the need to improve in the accuracy category and rely less on referrals. Responses with redeeming features include those that were partially accurate, but the DRSP took the time to steer the proxy to the correct answer. Pomerantz, Luo, and McClure (in press) suggested that an individual who contacted a reference service for assistance was less likely to understand how to gauge a response for accuracy and comprehensiveness. The students found two excellent responses that were accurate, documented, and effective; one response that was average, accurate, and effective; three responses that showed the need to improve in the accuracy category, but showed redeeming features; three responses that showed the need for improvement in the accuracy category; and five responses that showed the need to improve in the accuracy category and rely less on referrals (see Table 13).

Many of the DRSPs interviewed indicated that e-mail provided the librarian with additional time to work with the student's question. The DRSP had the opportunity to consult with colleagues, to check multiple resources, and to send an accurate and comprehensive response; however, study results showed that most DRSPs did not. They were not put on a moment's notice to answer the question. The additional time enabled an 
answer that had various scenarios based on the librarian's interpretation of the question. With the need to interpret different versions of the question, the DRSP often provided more information than the student needed did with an answer that was detailed, succinct, comprehensive, and complete with systematic instructions.

Table 13. Researcher/Student Comparison of Accurate and Satisfactory Responses to the 14 Questions

\begin{tabular}{lcc}
\hline \multicolumn{1}{c}{ Response } & Researcher & Student \\
\hline Excellent & 0 & 2 \\
Average, accurate, and effective & 2 & 1 \\
Improve accuracy, but redeeming qualities & 1 & 3 \\
Improve accuracy & 6 & 3 \\
Improve accuracy, rely less on referrals & 5 & 5 \\
\hline
\end{tabular}

\section{Do librarians respond to students in a timely manner?}

The opposing argument they offered was the opinion that timely reference transactions could not take place in an asynchronous environment. An interaction that went back and forth a few times delayed the resolution of the question. Many DRSPs appeared stressed by the time delay, as they believed it affected the relationship they developed with the student. "If the replies take too long you can lose the patron." The online interaction took much longer than the face-to-face interaction but students did not seem affected by the delay. One DRSP commented, "What is amazing to me is that students don't seem to mind if it takes much longer, apparently some are more comfortable in an environment where they can be anonymous and invisible, seems less threatening online to admit they don't know how to do something." 
The time it took the DRSPs to respond to the questions asked during the unobtrusive part of the study ranged from 6 seconds to 20 days. They answered $10 \%$ in less than one hour, $17.5 \%$ in one to two hours, $61 \%$ within 24 hours, $75 \%$ within 48 hours, $86 \%$ within 72 hours, $90 \%$ within 96 hours, and the remainder took from 5-20 days to answer the question. In terms of the average response time, DRSPs answered six questions (43\%) within 9-14 hours indicating an above average response time and eight questions (57\%) within 15-24 hours indicating an average response time. In terms of the average response time within regular business hours, DRSPs answered two questions (14\%) within 0-3 hours, 11 questions (79\%) within 3-12 hours, and one question (7\%) within 12-36 hours. DRSPs interviewed by the researcher indicated that a prompt reply was critical when dealing with students in the online environment. A comment that emerged from the interview suggested that the provider give the student periodic updates if the question could not be answered immediately.

\section{How do librarians respond to student inquiries? Do they ask additional or follow-up} questions to comprehend fully the student's research request?

In terms of the reference interview, the DRSPs interviewed had various opinions ranging from the asynchronous interview differing from the traditional one to an abbreviated version to a guessing game. Most agreed that the availability of asynchronous tools to offer reference service challenged the reference staff. Due to the asynchronous nature of e-mail reference, librarians needed to develop a more organized approach to their reference interviewing techniques. Within Florida's community college libraries, DRSPs had the opportunity to meet for Ask a Librarian training, Ask a 
Librarian synchronous reference service, regional College Center for Library Automation training, and statewide conferences.

Relying less on nonverbal cues and more on interpreting clues, the interviewing process became more instructional and dependent on professional judgment and expertise. The DRSPs needed to request feedback from the student. One DRSP said, "The librarian needs to be able to give an easily followed step by step response which probably spills over into face to face reference which can be beneficial." The absence of a face-toface interaction caused more than one DRSP to comment that traditional techniques, as basic as consideration for the student, were forgotten when a quick reply was sent to the student. While DRSPs might have suggested that students did not wish to develop an online relationship with a librarian, they believed the student expected a comprehensive response from the DRSP with the first e-mail.

Although student comments were fewer in number, they still ranged from poor to good (see Table 14). Overall, 12 student assessors found that the DRSPs provided good help, good answers, good searching, or good explanations. One of these assessors said, "Good enough for me! :)" leading the researcher to contemplate the satisfaction versus accuracy debate about reference service. Seven student assessors indicated the DRSPs to be friendly, nice, polite, or insightful individuals. Six student assessors commented about the amount of information conveyed through the response as being substantial, sufficient, or short. Five student assessors found the DRSPs to be helpful. Two student assessors appreciated a timely response by the DRSP or the amount of time spent by the DRSP responding to the question. 
Eight student assessors observed the lack of concern shown for the proxy, poor customer service given by the DRSP, and a lack of interest on the part of the DRSP. Six student assessors remarked on the DRSPs' impersonal tone. Six student assessors found the DRSPs to be less than helpful. Five student assessors criticized the length of time it took the DRSPs to respond to the virtual inquiries. Four student assessors criticized the responses as being hard to understand, vague, or short. Four student assessors noted incorrect answers. Three student assessors found the DRSP did not answer the virtual inquiry. Two assessors objected to the use of referrals and one noticed the lack of source information. One student assessor commented that an unsigned inquiry could have been attributed to anyone, not a DRSP.

Most of the DRSPs interviewed by the researcher agreed that the student's skills were difficult to determine in the online environment so assumptions could not be made about a student's level of technological knowledge or research capabilities. The DRSPs agreed that a student who was able to locate the "Ask a Librarian" Web page, input a question, and send an e-mail had at least rudimentary computer skills. The assumption could not be made by the DRSPs that all students possessed research skills so they approached each question from the perspective that the skills were not sufficient. Results showed that the DRSPs used the opposite approach. Other DRSPs suggested that students with poorly formulated research questions might point toward inadequate research skills. Poor research skills appeared more problematic to the DRSPs than inadequate computer skills. They said they provided responses to even those students with observable technological or research difficulties; however, detailed instructions and comprehensive resource 
information accompanied answers. Results showed that the DRSPs disregarded their own guidelines.

The results from the unobtrusive study indicated that the DRSPs appeared to overestimate the skills of the students by suggesting resources that required additional reading, technical, navigational, or research skills. Instructions that were vague or complicated could have possibly hindered student success. The DRSPs referred the proxy to a variety of resources, including books, newspapers, databases, and Web sites. Information on locating the online catalog or online databases was absent from many responses. The proxy rarely received information about obtaining or using the library barcode or password. Many DRSPs omitted login instructions from their responses. Suggestions for formulating suitable search strategies were rare amongst the responses. Instructions for utilizing online databases or other resources were deficient.

The failure to grasp the preference of the student to work in the virtual environment appeared evident as the student was often referred to the telephone or the library to answer questions that might take the DRSP time to construct, i.e., database instructions, statistical responses, etc. The infrequent attempts at conducting a virtual reference interview were evident. While initial assistance or direction was provided, a lack of follow-up appeared obvious. The proxy sometimes received the same instructions to follow that he or she had already tried. In essence, the DRSP pushed back the question to the proxy in the guise of an answer. DRSPs also forgot to attach documents or the list of recommended Web sites. 
Table 14. Student Assessors' Observations

\begin{tabular}{cl}
\hline Number of Responses & \multicolumn{1}{c}{ Positive Comments } \\
\hline 32 & Total \\
12 & Good help, answers, searching, explanations \\
7 & Friendly, nice, polite, or insightful \\
5 & Substantial, sufficient, short answers \\
2 & Helpful DRSPs \\
\hline Number of Responses & Timely response or time spent by DRSP \\
\hline 42 & Total \\
8 & Lack of concern, poor customer service, or lack of interest \\
6 & Impersonal tone \\
6 & Less than helpful \\
5 & Length of time taken \\
4 & Responses hard to understand, vague, or short \\
4 & Incorrect answers \\
3 & Answer not give \\
3 & Object to referrals \\
1 & Lack of source info \\
2 & Unsigned inquiry attributed to anyone \\
\hline &
\end{tabular}

DRSPs suspicious of the proxy threatened the unobtrusiveness of the study. These wary DRSPs questioned the proxy's personal e-mail address, the proxy's use of a personal e-mail address rather than the institutional-issued e-mail address, the proxy's affiliation, and the number of recent e-mail inquiries from unusual e-mail addresses. One DRSP sent both the proxy and a colleague a message about the reference inquiry coming from a questionable e-mail address. The librarian opted to "dump" the request. The response indicated that the matter would be reported to a third colleague. Another DRSP 
told the proxy to contact his or her local librarian for assistance. In addition, another reproached the proxy for using his or her own e-mail address instead of a college-issued address.

Poor reference skills appeared evident for most questions. The DRSPs failed to read a number of the questions closely resulting in poor responses. The medical, government, and statistics questions emerged as problematic. DRSPs diagnosed a medical condition, but then failed to read correctly a question about a pharmaceutical product. Many DRSPs failed to consider federal or state agencies as a source for information. Suggestions to utilize older, traditional sources for current statistical questions reduced the level of accuracy. While these questions appeared more difficult to answer during a first reading, the DRSPs failed to obtain assistance from colleagues for questions beyond their expertise. The ability to take the time to respond to an inquiry was one of the benefits of asynchronous reference. The failure of the DRSP to stay abreast of current events weakened the perception of his or her reference skills. Referring students to physical libraries when the answer was available on the Web reduced the effectiveness of the DRSP, too.

The proxy was often referred to a resource or a person; however, the DRSP neglected to consult the source or the individual prior to referring the student. The DRSPs also referred students to online help sites or citation generators without verifying the accuracy of the resource. The two questions dealing with the APA and MLA citation formats provided primary examples. Some of the referring Web pages contained so many resources that a student would be completely overwhelmed by the volume of resources and frustrated by his or her inability to determine which source to check first. For each of 
these referrals, the proxy assumed the burden of locating the source and checking for himself or herself.

The availability of interlibrary loan, reciprocal borrowing among community college and university libraries, and the ability to search LINCC for "ALL COLLEGES" appeared to be forgotten by most of the DRSPs. DRSPs failed to understand specific login or access problems and offered no real assistance to the proxy. Their ignorance about access problems appeared troublesome since access to online databases within the community college library system had been available for a number of years. The inability to resolve access problems was found to be problematic with most libraries.

DRSPs interviewed by the researcher suggested the approach to the reference inquiry as important for a successful transaction. Their suggested approach differed from actual responses. Responses focused on a beginning to end method of handling the student's inquiry. Respondents believed the use of salutations, greetings, and sign-offs critical to developing an online relationship. They further suggested the use of name, title, phone number for both the provider and the general reference desk, and the e-mail address of the provider. Many libraries had the student's response sent to a folder viewed by all of the providers. They also recommended the use of the personal touch to establish rapport. One provider asked about unusual e-mail addresses and another added personal perspective into the response when warranted. Techniques included imbuing a sense of humor and a cheerful, chatty, friendly tone into the response. They also advocated the use of encouraging language.

The DRSPs interviewed also indicated that their response aided in the communication between the DRSP and the student. DRSPs recommended that responses be clear and 
complete, including as many options as the provider believed would answer the question. The suggested language from a number of providers was informal and easily understood by many readers. They advocated a tone of voice that was cordial, helpful, natural and not canned, and friendly. The response needed to include detailed steps with resources of reputable quality. The suggested closing was a request for follow up questions and an expression of thanks for using the service.

Do these services enhance the student's online learning community? Does the librarian initiate an open and inviting dialogue? Does the librarian attempt to personalize the experience for the student? Does the librarian invite the student to use the service again should additional questions arise?

Most of the DRSPs interviewed by the researcher agreed that the library was a student support system. The role of the library, both online and on campus, was critical to student success. The library's services and resources were included in DL programs at the responding institutions. Some librarians even taught classes in the online environment. They were uniquely positioned to assist students from two perspectives, that of an instructor and that of an information professional. For those providers who had taken DL classes, the perspective was broadened to even that of a student. They suggested that their role was to provide timely personalized one-on-one guidance and direction in a sometimes-confusing online environment.

The role of the digital reference provider was similar to the role of the reference librarian working with students in a face-to-face environment. DRSPs offered students service in the online environment equivalent to what their institutions offered in person. 
Students were guided through the research process albeit the environment was electronic. They were taught how to phrase a research question, select or navigate electronic tools, and evaluate results. The online environment slowed down the process.

When interviewed by the researcher, the DRSPs said that to develop an online rapport with the student, the DRSP must use an encouraging tone to invite additional questions or volunteer to assist the student further with the request. Some providers supported the use of encouragement through telephone calls, face-to-face appointments, or synchronous chat sessions. The need to thank the student for his or her patronage and to invite the student to utilize the service again surfaced in their comments. Pomerantz, Luo, and McClure (in press) reviewed the librarian's enthusiasm as part of their 2004 unobtrusive study of virtual reference and identified librarians as "very unenthusiastic" (p. 22).

They suggested that their inability to establish any type of relationship with the student was due to the online environment and the manner in which their digital reference service was set up. The pervading impression garnered from some of the providers was that few online encounters engaged students.

In terms of communication during the unobtrusive portion of the study, there was no significant difference between the researcher's scores and the students' scores in 12 of the 14 questions. The researcher found that in all 14 questions, some online communication skills were unsatisfactory. The student assessors found in 10 questions that some online communication skills were unsatisfactory, in three questions that online communication skills were adequate, and in one question that the online communication skills were inadequate. Inadequacies appeared in greeting the student, using the student's name, interest, repeating the reference question, asking questions, fully explaining the 
answer, providing steps for independent research, satisfaction with the answer, invitation to utilize the service again, closing the transaction, and indicating professional position.

\section{Impact of Institutional Size}

The researcher reviewed institutional policies, staffing, response rates, communication, accuracy of the responses, and the timeliness of the responses for all sized institutions. Based on the responses given during the interview and the results of the unobtrusive portion of the study, institutional size influenced specific aspects of the overall study. Not one group emerged in terms of excellence due to the lower than average response, accuracy, and quality rates.

Six of the 23 DRSPs acknowledged having written digital policies in place, including $13 \%$ of the large-sized institutions, $8.7 \%$ of the medium-sized institutions, and $4.3 \%$ of the small-sized institutions. The low percentage of institutions without policies indicated a lack of importance in the quality of service provided and the impact of reduced quality on the student; however, the percentages were too low to infer that size affected outcome. Established policies and assessments of service might have improved the lower than average statistics plaguing each size group and the system as a whole.

Staffing of institutional digital reference service points showed that $48.48 \%$ of the 99 DRSPs came from large-sized institutions, 36.36\% from medium-sized institutions, and $15.15 \%$ from small-sized institutions. While students using highly staffed digital reference services appeared to have a greater likelihood of connecting with a DRSP who provided effective assistance in an asynchronous environment; the less than average 
unobtrusive results, as well as the lack of institutional policies regarding service and assessment, reduced this probability for each size group.

The response rate by institutional size varied significantly (see Table 15). The 10 small-sized institutions responded 64 times to a potential 140 inquiries for a return rate of $46 \%$. The nine medium-sized institutions responded 85 times to a potential 126 inquiries for a return rate of $67 \%$. The nine large-sized institutions responded 91 times to a potential 126 responses for a return rate of $72 \%$. The likelihood of reaching the $55 \%$ accuracy statistic for each size group, as suggested by Hernon and McClure's 1986 study, was reduced drastically because $100 \%$ of the proxy's questions were not attempted.

Table 15. Number of Institutions (by Size) Answering each Question

\begin{tabular}{lccccccccccccccc}
\hline \multicolumn{1}{c}{ Size } & Total & $\mathbf{1}$ & $\mathbf{2}$ & $\mathbf{3}$ & $\mathbf{5}$ & $\mathbf{6}$ & $\mathbf{7}$ & $\mathbf{8}$ & $\mathbf{9}$ & $\mathbf{1 0}$ & $\mathbf{1 1}$ & $\mathbf{1 2}$ & $\mathbf{1 3}$ & $\mathbf{1 4}$ & $\mathbf{1 5}$ \\
\hline Small & 10 & 3 & 3 & 4 & 4 & 6 & 4 & 4 & 5 & 4 & 5 & 6 & 5 & 6 & 5 \\
Medium & 9 & 5 & 6 & 6 & 6 & 8 & 5 & 7 & 6 & 6 & 4 & 6 & 7 & 6 & 7 \\
Large & 9 & 7 & 7 & 7 & 5 & 7 & 7 & 7 & 8 & 6 & 5 & 7 & 7 & 7 & 4 \\
\hline
\end{tabular}

In terms of the communication between the proxy and the DRSP, the researcher found that some online communication skills were unsatisfactory in 13 of the 14 questions for all institutional sizes. Student assessors differed in their interpretation of communication skills as they identified two questions from the large-sized institutions, three questions from the medium-sized institutions, and five questions from the small-sized institutions where communication skills were adequate. The student assessors scored the remainder of the responses as unsatisfactory. The researcher found that $93 \%$ of the responses were unsatisfactory for all size groups and $2.3 \%$ of the responses were adequate for each of the size groups. The student assessors found that $76 \%$ of the responses were unsatisfactory 
for all size groups. Small-sized institutions accounted for $12 \%$ of the adequate responses, medium-sized groups for $7 \%$ of the adequate responses, and large-sized groups for $5 \%$ of the adequate responses. Established policies and assessment of services might have improved adequacy of the responses plaguing each size group and the system as a whole.

The quality of the responses combined with a willingness to return to the DRSP for assistance varied. The researcher found that the small-sized institutions e-mailed more excellent $(7 \%)$ and average (14\%) responses compared to the excellent $(0 \%)$ and the average $(0 \%)$ responses from the medium-sized institutions, and to the excellent $(0 \%)$ and average (7\%) responses from the large-sized institutions. The medium-sized libraries e-mailed more responses (29\%) that needed improvement, but showed redeeming features. The small-sized institutions e-mailed $7 \%$ of the responses from this category and the large-sized institutions e-mailed 14\%. The small-sized institutions e-mailed more responses $(36 \%)$ that needed improvement in the accuracy category, whereas, the medium-sized and the large-sized institutions both e-mailed 29\%. The large-sized institutions e-mailed more responses (50\%) that needed to improve in the accuracy category and rely less on referrals. The medium-sized institutions and the small-sized institutions e-mailed $43 \%$ and $36 \%$, respectively. The small-sized institutions provided more quality responses than the other sized groups. The medium-sized institutions and the large-sized institutions followed second and third, in that order.

The student assessors' found that the small-sized institutions and the medium-sized institutions each e-mailed the same number of excellent (7\%) responses while the largesized institutions e-mailed none. Each of the three sized institutions e-mailed the same number of average $(7 \%)$ responses. The medium-sized libraries e-mailed more responses 
(36\%) that needed improvement, but showed redeeming features. The small-sized and the large-sized institutions each e-mailed $29 \%$ of the responses in this category. The largesized institutions e-mailed more responses (43\%) that needed improvement in the accuracy category, whereas, the small-sized institutions e-mailed 36\% and the mediumsized institutions e-mailed 29\%. The small-sized and the medium-sized institutions each e-mailed $21 \%$ of their responses that needed to improve in the accuracy category and rely less on referrals, while the large-sized institutions e-mailed $14 \%$. The medium-sized institutions provided more quality responses than the other sized groups. The small-sized institutions and the large-sized institutions followed, correspondingly.

The amount of time taken to respond to the proxy's inquiries fluctuated by the size of the institution. The large-sized institutions responded faster than the medium-sized institutions and the small-sized institutions, in that order. The timeliness of their responses occurred for both time of day and business hours.

\section{Researcher's Observations}

The researcher had been a research specialist and academic librarian for 25 years, an asynchronous digital reference service provider for 14 years, and a distance learning instructor for 9 years. The researcher also had experience working with the DRSPs within Florida's community college library system and found them to be passionate about, involved with, and concerned about providing outstanding library service to students within the system. The results of the study disappointed and troubled the researcher.

The number of providers appeared adequate for each of the size groups. Ninety-nine percent of the DRSPs possessed master's degrees in the field. Their longevity of 
experience averaged 14.5 years. Only $60 \%$ of the student assessors indicated a willingness to return to these DRSPs for assistance further exacerbating the ongoing battle to maintain a customer base against the popularity of the Internet.

The communication techniques exercised by the DRSPs were substandard, yet they believed communication skills critical to developing an online relationship with the student. They considered the library a bridge between the student and academic success. The study considered 15 areas for assessing the communication between the DRSP and the proxy. The researcher equated scores of $69 \%$ or lower to a grade of $\mathrm{D}$ or $\mathrm{F}$, similar to the scores community college students themselves earned in class. In only five categories were $70 \%$ or more of the responses answered with acceptable communication skills.

Ten areas fell below the $70 \%$ mark. The responses lacked the common courtesy extended to students during face-to-face transactions. Most DRSPs failed to provide the proxy the steps needed to duplicate their search strategies thereby eliminating opportunities to develop information literacy skills. DRSPs also exhibited a lack of professionalism by sending responses with misspelled words or poor grammar. The DRSPs were careless in their handling of the reference queries.

The DRSPs returned only $61 \%$ of the proxy's requests. The number of responses that were wholly accurate with or without sources was low at $28 \%$. The number of responses that were partly accurate with or without sources was even lower at $27 \%$. The accuracy score paled in comparison to the $55 \%$ accuracy figure established by Hernon and McClure (1986). The low accuracy rate was puzzling for a profession that strived to retain its value to the consumer in an Internet-based society. 
Possible explanations for the low accuracy scores might have been the personal philosophy or available time of individual DRSPs. Their responses were often short and hurried despite the additional time that asynchronous service allowed the DRSP. They provided monitored and unmonitored referrals, as well as source information only, despite the interview comments given to the researcher about the comprehensive replies sent to students.

Most DRSPs did not consult colleagues for the more difficult questions, yet they struggled with government document and statistics questions. Basic library services, such as interlibrary loan, were not suggested, as often as they should have been. Current events questions led the researcher to assume that the DRSPs did not follow local news. The DRSPs neglected to check the accuracy of their responses prior to sending them to the proxy or they eliminated information critical to the proxy. They utilized outdated reference and Web sources. The citation generators were flawed, yet the DRSPs suggested their use. They also relied on home-produced and error-filled guides for APA and MLA citations rather than primary sources.

In some instances, the digital reference access links were broken or the e-mail address for service was incorrect. The DRSPs lacked expertise in providing an explanation of the authentication question. Most neglected to provide the proxy information on the barcode, database Web page, login instructions, and suggested search strategies. Personal information sought about the proxy through a number of Web forms appeared to be unwarranted, especially since many DRSPs agreed to support questions asked of their services by all users and most did not have digital reference policies in place. 
The DRSPs assumed the technology and research skill levels of the students to be at a higher level. Given that many community college students arrived as freshmen with deficient reading, writing, and mathematics skills, the likelihood of these same students interpreting or following referrals or suggestions of DRSPs appeared unlikely. According to Parsad and Lewis (2003), 42\% of college freshmen entering public two-year institutions enrolled in remedial courses for reading, writing, or mathematics in 2000, up from $40 \%$ in 1995 (p. 18). From this same group, 20\% had reading deficiencies and 23\% lacked adequate writing skills (p. 18).

The proxy was oftentimes forgotten or ignored, given responses appropriate for an experienced researcher, or treated as if the question asked was unsuitable for the online environment. DRSPs did not survey the satisfaction of their patrons nor did they assess the adequacy of their responses. A comment made by one of the interviewees summed up the need for accurate and comprehensive asynchronous reference service.

If a student has a legitimate question, the librarian should answer the question. Students cannot succeed if their support systems reject them. If a library restricts service, perhaps its staff should not offer online reference assistance. More and more students take online classes. If libraries cannot improve or augment their services, let libraries who believe in comprehensive service, conduct it. If the question is beyond the scope of the library providing the service, the library staff should intermediate a referral on the student's behalf. 


\section{Implications}

This study provided an overview of reference services from the digital perspective. Digital reference service provided a challenge to libraries as it is a relatively new service with a slowly growing clientele. The majority of the studies conducted to date have been done in-person or by telephone, so this study helped to establish tangible data on the topic. With both asynchronous and synchronous digital reference services available and in effect, the literature suggested that research studies would augment the mostly anecdotal results written about the adequacy and success of digital reference services. Hernon and McClure (1987a) suggested that studying reference service also "provides empirical data from which decisions and managerial strategies can be based" (p. 105).

The results of the study addressed the adequacies and inadequacies of the DRSPs' responses to reference inquiries and the communication techniques initiated by them. An analysis of the data might help to justify improvements to digital reference services and to the library support services provided to DL students. Katz (2001) stressed that technology allowed for efficient and accurate responses and librarians must improve information retrieval skills. Lankes (2004) suggested the need to meld the concept of customer service with digital reference. Janes (2004b) believed that digital reference should offer patrons more than ready reference responses and the DRSPs should use fully the skills and resources available. Frank (1998) said that ready reference type questions would not suffice because DL students needed more in-depth service to suit their needs. The results showed that DRSPs have much to learn about the online environment and DL students. The interview responses provided by the DRSPs facilitated a comparison among 
actual service provided, the documented procedures and policies, and the perceptions of digital reference service as identified by select DRSPs.

The "accuracy, fill rate, quality, service and satisfaction" of reference service had not been adequately addressed in terms of standards contributing to service that is "goodenough" for patrons (Coffman \& McGlamery, 2000, p. 66; Cullen, 2002, p. 30). With accuracy statistics hovering around the 55\% mark and this study showing a lower rate of success, the implication existed that librarians must improve information retrieval skills, instructional skills, and service criterion. Dilevko (2000) suggested librarians take personal responsibility for improving their reference skills. The literature also implied that poor service equaled a lowered professional image and the loss of confidence in reference service (Blenkinsopp, 1992; Farmer, 2005; Jirjees, 1981). Jirjees attributed inaccurate responses to a failure in using library resources or subject specialists, a purported lack of time, the inability to use library resources, a misinterpretation of the student inquiry, negative staff attitudes, and pressure to respond to the inquiry (pp. iv-v). The library and information science profession should have worked on the accuracy problem since it appeared to be decades old (Dilevko, 2000, p. 184). The results might have helped to determine the types of service and resources that would best help professionals assist students in a virtual environment (Horner \& Michaud-Oystryk, 1995).

The results of the study helped to show that the unobtrusive methodology still offered a reliable way of analyzing reference services. Hernon and McClure (1987a) concluded that the benefits resulting from unobtrusive studies included assessing the effectiveness of reference services, improving effectiveness of reference services, improving the methodology taught in graduate school with regard to reference interview techniques, and 
improvements to unobtrusive testing techniques. The ability to conduct an unobtrusive study within the digital environment strengthened the use of the methodology for yet another mode of service delivery. The unobtrusive in-person and telephone reference studies mentioned in the literature review suggested that challenges already existed in the provision of reference services. This study added another challenge to the list, that is, the provision of reference service within the digital environment.

Libraries lacked standard criteria for offering quality digital reference services thereby impeding the ability to determine effective practices. The analysis of results might have influenced the standards for service needed by the profession. Without accepted standards usable by the profession, quality remained difficult to ascertain. While digital reference might be in its infancy, baseline data helped to determine current levels of service as well as a starting point for the discussion of the extent of services (Novotny, 2001; Tennant, 2003; Wasik, 2003). Standards helped provide the evidence that an institution offered students a quality learning experience leading to academic success (Banta, Lund, Black, \& Oblander, 1996; LaPadula, 2003).

\section{Recommendations}

The study resulted in recommendations for further study in the areas of the digital environment, unobtrusive methodology, standards, accuracy, measurements, online relationships, training of DRSPs and students, institutional responsibility, and promotion. 


\section{Digital Environment}

Since many libraries established digital reference services without forethought to goals and assessment, the literature did not adequately address the effectiveness of digital reference service (Lankes, Gross, \& McClure, 2003). Anecdotal observations provided discussion points, but the development of standards, goals, and assessment tools usable by the majority of providing libraries appeared to be needed as a critical step in $21 \mathrm{st}$ century library services. Ward (2004) believed digital reference service "should be comparable to that which patrons have traditionally expected from reference desk staff' (p. 53). The creation of guidelines might be integrated into current practices or burgeoning practices (Barkley, 1998).

\section{Unobtrusive Methodology}

"Unobtrusive testing of reference services is not an end unto itself but a means toward improving the quality of library services" (Hernon \& McClure, 1987a, p. 104). Ward (2004) also found that unobtrusive techniques worked well within the digital environment. The results of this study indicated that unobtrusive techniques worked well in the digital environment. The researcher recommended replicating this study with another statewide community college system and interviewing the library administrator after the unobtrusive study had taken place. Discovery by the subjects remained the main threat to the researcher. For the past three decades, the profession supported the technique with results showing $50 \%$ to $62 \%$ of inquiries being answered correctly (Hernon \& McClure, p. 3). The technique provided realistic responses, posed no true threat to the subjects, and was inexpensive to conduct (Crowley \& Childers, 1971). The use of 
unobtrusive testing to review new librarians was recommended as a method of gauging skill and correcting mistakes before they became professional habits.

Jensen (2004) suggested that librarians utilize action research instead of unobtrusive testing. The involvement of librarians in the study lent itself to feedback, improved service, and cooperation (p. 148).

\section{Standards}

Researchers agreed that the need for standards for service existed, but the lack of national standards created a void (Horner \& Michaud-Oystryk, 1995; Wasik, 2003). Ryer and Nebeker (1999) commented that digital reference was a relatively unknown area further emphasizing the recommendation to develop national standards while the service is in its infancy. While individual institutions might have created their own standards, local standards were often drawn from established national standards. With the increased number of collaborative endeavors, the need for standards increased. Powell and Bradigan (2001) advocated standards for assessment, guidelines, clientele, and disclaimers. The need for local libraries to evaluate local services existed. Evaluation that occurred on an ongoing basis could be used for future comparative studies and these comparative studies used to improve the quality of digital reference services.

\section{Accuracy}

The debate about the accuracy of reference responses persisted, although the newest environment was a digital one. Continued attention to the adequacy of responses was warranted. One of the student assessors for this study claimed the answer provided was 
“Good enough for me!" Student satisfaction was only one of the criteria needed for an adequate and accurate response. The DRSP's competence and expertise affected the success or failure of a transaction so the continued education and skill building of DRSPs was warranted due to the study results (Hernon \& McClure, 1983). DRSPs could build skills individually, institutionally, or through professional education.

Researchers considered referrals to be inaccurate responses since DRSPs could not monitor student behavior easily (Crowley \& Childers, 1971; Paskoff, 1991). An additional recommendation suggested that DRSPs include comprehensive contact information when referring a student to a person or a source, as well as first determining that the referral answered the question (Jahoda \& Braunagel, 1980).

\section{Measurements}

The interview with the DRSPs pointed toward inadequate means of data collection as a way of assessing adequate service. Ratcliff (1996) stipulated the use of assessment to improve services for accountability purposes. It provided evidence that an institution offered students a quality learning experience leading to academic success (Banta, Lund, Black, \& Oblander, 1996; LaPadula, 2003). Limitations in service prevented the growth of a necessary service. McClure, Lankes, Gross, and Choltco-Devlin. (2002) recommended the use of field-test measures. The use of log analysis to track responses for further studies was also recommended (Gross, McClure, Hodges, Graham, \& Lankes, 2001). Rodman (2003) recommended tracking responses for a return on investment review, as well as a time efficiency review. Based on the turnaround time from this study, Rodman's recommendation had merit. Saxton, Kaiser, and Reichert (2004) suggested the 
use of peer review to improve digital reference transactions. Transcripts appeared to be another way to track and statistical count transactions. User and non-user comments generated qualitative measurements that enhanced statistical measurements.

\section{Online Relationships}

Since the digital environment created a non-visual and a non-verbal dynamic between the DRSP and the student, the profession needed to address the development of online relationships to humanize the experience for both the student and the DRSP (Gothberg, 1973; Radford \& Thompson, 2004). Lankes (2004) suggested the need to meld the concept of customer service with digital reference and to develop the relationship between DRSPs and the members of the digital community. Hodel (2004) suggested training as a critical element in providing service. Bell and Levy (2004) recommended training librarians to adapt verbal signals for the digital environment. Furthermore, librarians should not be given digital reference assignments unless their "desired reference behaviors" had been established and they could utilize the reference tool set, emphasize quality service, and realize the patron's position (p. 9).

Radford and Thompson (2004) shared the results of a study dealing with relational dimensions in chat reference and they identified rapport building, deference, compensation for lack of verbal cues, greetings, and closings as critical to facilitating communication between the DRSP and the student. The relationship might have been developed through enhancements to the curriculum in graduate school, "Ask a Librarian" training, focus groups with students, or a tiered system of response. A tiered response system enabled a second set of eyes to review the response to the inquiry to identify 
problematic language or an inadequate response. The use of digital photos on the library's Web page might have helped the students connect with the DRSPs (LoveRodgers, 2001).

Duff and Johnson (2001) suggested further study to document how students asked questions remotely. The researcher concurred, based on the study results for those questions actually asked by DL students at the researcher's institution. A checklist, not a canned response, also tied to standards, might have helped DRSPs remember the techniques to communicate effectively or to build an online relationship with a student. The DRSPs interviewed by the researcher discussed the student's need being unsuitable for the online environment. The recommendation to develop an understanding of distance learning education might have helped DRSPs to realize that all questions asked in the DL environment, with the exception of those questions deemed inappropriate in any venue, were acceptable since the student was taking online classes and the IHE provided support for the student in this environment.

\section{Training of DRSPS}

One of the results from this study had been the identification of suggested areas for additional training. Lankes, Janes, Smith, and Finneran (2004) believed that adequate training guiding librarians for their new role in the digital environment was taking time to become integrated into graduate education and professional development training opportunities. Salem, Balraj, and Lilly (2004) discussed basic reference skills, digital techniques, an understanding of reference policies, and the analysis of reference transcripts as components in digital reference training. Harris (2004) referred to Janes' 
curriculum for a University of Washington course on digital reference. The course introduced graduate students to the reference transactions, literature, online tools, searching techniques, and hands-on practice within the digital environment.

A knowledge audit might have supplied additional areas for training to individual institutions. Additional training coupled with future unobtrusive testing of reference might have led to more effective reference service. Abels and Ruffner (2004) advocated hands-on training with a required set of skills for the digital environment. Dilevko (2000) supported the continued development of skills with periodic retesting. Proposals to certify, recertify, and continue the educational process of professional librarians remained current in the professional literature (Lester \& Van Fleet, 2003; Milam, 2005; Moran, 2003; Ojala, 2003). Credentialing, certification, and recertification already occurred in the health professions and other licensed occupations.

The researcher supported the recommendation of researchers advocating the improvement of graduate education to improve the methodology taught in graduate school with regard to interview techniques, question negotiation, and search strategies with a further recommendation to add interpersonal communication within the digital environment (Farmer, 2005; Feili-Tu, 2004; Hernon \& McClure, 1987b; Lee, 2004). Abels and Liebscher (1994) also suggested introducing effective asynchronous reference services to students while they were in graduate school for library and information studies coursework. Daniel (2003) and Staley (1998) supported continued education and training. Staley considered further education an opportunity for staff development.

The development of a best practices training manual, usable in both a formal and an informal setting, was yet another recommendation emerging from this study and 
supported by the literature (Daniel, 2003; Morin, 2004; Moss, 1997; WCET, 2003b). Transcripts might have been used as a teaching tool. They might also be used to develop a best and worst practices manual. A review of responses made by other librarians prior to deleting them or creating a folder of excellent answers might have supplemented a best practices manual (Anderson, Boyer, \& Ciccone, 2000). With asynchronous reference relying more on "best guesses," "mind reading," and interpretative techniques, the use of an informational template may have helped the DRSP more definitively determine the needs of the student. This template might be located online and in a training manual.

Dilevko advocated tying performance to compensation. Anderson, Boyer, and Ciccone (2000) concurred with the need for additional training; however, they believed follow-up questions might determine whether the quality of service had improved. User comments incorporated into some of the teaching tools, perhaps as a lesson, also provided insight into the student experience. The final recommendation for this section was that DRSPs take an online class and experience the digital environment as a student.

\section{Training of Students}

The literature review prompted several training recommendations. Students might have failed or struggled within the digital environment because they neglected to learn about the support services available to them as DL students. The use of e-mail lecturers, as suggested by the University of Minnesota Libraries (1998), warranted further study. Studies dealing with the adequacy of orientation sessions in both the digital and the traditional environments appeared warranted. Stephens (1996) concurred with this recommendation since students did not appear to make use of the services already 
available to them. Lorenzetti (2002b) believed that orientation helped students to understand the resources and technology of library tools; however, further study might have supported the statement. Another recommendation suggested training students, especially those new to DL courses, to express themselves succinctly in an online environment (WCET, 2003b). The inclusion of contacts, resources, online communication skills, downloading information, and the development of student cohorts for persistence was yet another area for consideration (Astin, 1993; Scagnoli, 2001). Further recommendations involved the use of learning styles for a personalized approach to the development of digital reference service (Massey-Burzio, 2002).

\section{Institutional Responsibility}

Since DL students were not the only individuals who might take advantage of digital reference services, Kern (2004) suggested training reference staff about both the service and the policies. Salem, Balraj, and Lilly (2004) stressed the need for an organizational culture that integrated training for the digital environment. Staff development opportunities or formal education were two avenues for developing this culture. The inclusion of the DRSP in the curriculum design of DL courses, including those that utilize course management software, might have provided an avenue for the DRSP to share instruction with the student (Buehler, 2004; Foster, Evans, \& Lott, 1997; Stephens, 1996). Singh and Pan (2004) suggested threaded discussions, live chat, e-lectures, and personal communication as avenues for improved delivery of course information ( $\mathrm{p}$. 303). Library input might have also been made using these techniques. Library access, library services, login instructions, tutorials, suggested search strategies for particular 
course assignments, and recommended databases were not presented adequately to the proxy, but they might have been addressed as part of the course design (Markgraf, 2002). A future recommendation for continued research suggested that DRSPs co-teach specific lessons or visit online classes to both establish a relationship with the students and to provide some online instruction (Lawson, Lillard, Antrim, \& Morgan, 2000).

Effective Web pages served the student (Daniel, 2003; Fagan \& Calloway, 2001; Mudrock, 2002). Some of the Web sites to which the proxy was directed were overwhelmingly detailed and poorly designed, and a number of them contained dead links. Chat sessions or video conferencing within a DL course might have positively affected the relationship between the student and the DRSP (Collins, Schuster, \& Ludlow, 2002; Goetsch, 1995; Tennant, 1999). If students were mentored within the online environment, the DRSPs contributed to student success and institutional accountability (Jesudason, 2000). The final recommendation resulting from this study in this category was to embed a librarian in each DL course much as journalists were embedded into military units during the Iraq war.

\section{Promotion}

Introducing students to the services available to them through the online environment may have assisted in the promotion of a slowly developing service. Lee (2004) advocated for adequate marketing. Students could not utilize an unknown service. Thomsett-Scott (2004) recommended the use of memorable slogans as a marketing tool. DRSPs interviewed indicated that their digital reference service was just a small piece of what they did and, in some cases, not worth assessing or measuring. With campus-based 
reference transactions dwindling and DL programs and courses increasing, publicizing library services strengthened this support service for DL students. Tools for promotion included, but were not limited to, campus publications, library instruction, workshops for college staff and for students, library handouts, Web page announcements, electronic message boards, local newspapers, newsletters, signs, word of mouth, public service announcements through the radio station, and sending a message to all students and faculty with e-mail addresses (Casey, Sochrin, \& Race, 2002; Fishman, 1998, p. 5; Ryer $\&$ Nebeker, 1999, pp. 29-30). Frank (1998) placed the service's e-mail address in the campus telephone directory, local network, campus newspaper, library's Web page, bookmarks, business cards, remote logon sites, faculty staff orientations, and library instruction sessions (p. 8). Studies addressing the value of each method of promotion may have identified the top tools.

\section{Summary}

The U.S. Department of Education's National Center for Education Statistics (NCES) (2002) found DL courses and programs offered through the Internet tended to be more popular with postsecondary students than the other DL options of television or audio classes offered in live or prerecorded formats. Dunn (2001) estimated that within the next 10 years, nearly $95 \%$ of all educational providers recognized by the U.S. Department of Education would offer digitally enhanced instruction (p. 29). Online courses eased overcrowded classrooms and parking facilities; reduced time spent on campus; enabled shared resources at various campus locations; expanded course offerings beyond the physical limits of the campus; provided students with another mode of learning; and 
helped students to balance the responsibilities of home, school, and work (Florida Department of Education, 2002b).

Colleges and universities initiated the offering of DL programs; however, the capacity for these institutions of higher education to provide adequate support services remained an issue (Dinwiddie \& Lillard, 2002; Lefor, Benke, \& Ting, 2003; Ludwig, 2002). Literature suggested that adequate support offered a quality indicator and affected the success of an institution's DL program and its commitment to the DL student (Floyd \& Casey-Powell, 2004; McLean, 2000). To ensure adequate programs and courses in the Web environment, the nation's regional accrediting associations for colleges and universities stipulated that support services provided to DL students be comparable to those offered to campus-based students (Lebowitz, 1997). Student support services included library services and resources, admissions, financial aid, tutoring, registration, student rights, advising, technical support, health services, bookstore, and other services and resources. These same accrediting associations also mandated the use of systematic and ongoing assessment of support services to assure continued quality and adequacy.

During the 1990s, more libraries began offering DL resources and services comparable to those provided to campus-based students (Mahony, 1993). Library services available to DL students included, but were not limited to, digital reference and instructional services, remote access to online research tools, database and research tutorials, interlibrary loan, and document delivery. Digital reference, through both asynchronous and synchronous means, became one response to accreditation requirements and ALA's recommendations. Researchers defined digital reference as a Web-based service providing mediated reference assistance to clients within an online 
environment (Lankes, 2004; Lankes, Gross, \& McClure, 2003; Pomerantz, 2003; Smith, 2003; Whitlatch, 2003).

Lankes, Gross, and McClure (2003) contended that libraries established digital reference services without forethought to goals and assessment. Tennant (2003) and Wasik (2003) attributed the lack of assessment research to digital reference being in its infancy. Novotny (2001) stressed the need to conduct evaluations of new services to assess the level of service, as well as the extent of services provided. Despite the documented need to assess digital reference services routinely, the literature described mostly anecdotal observations and did not adequately address the effectiveness of digital reference service (Abels, 1996; Barcellos, 2000; Bristow \& Liu, 1999; BushwallowWilbur, DeVinney, \& Whitcomb, 1996; Clarke \& Brinkley, 2000; Garnsey \& Powell, 2000; Gross, McClure, \& Lankes, 2001; Janes, Carter, \& Memmott, 1999; Roysdon \& Elliot, 1988; Schilling-Eccles \& Harzbecker, 1998; Whitaker, 1989).

Some researchers remained bothered by inconsistencies in service (Brownlee \& Ebbers, 2002; D'Angelo \& Maid, 1998). Hernon and Whitman (2000) pointed out that asynchronous reference services, the more established of the digital library services, needed further analysis to assess its effectiveness. Hastings and Tennant (1996) found that no rules existed and their development occurred as services progressed. Morin (2004) and Barkley (1998) believed that the next step was to create and integrate guidelines into library practices. Santovec (2002) identified the use of evaluations to measure success in DL programs.

Kasowitz, Bennett, and Lankes (2000) found that little had been done to delineate standards for digital reference service and regular evaluation helped determine quality, 
accuracy, efficiency, and observance of established policies (p. 360). A primary need for standards was the user's expectation of a comprehensive, timely, and accurate response. Previous studies showed that only about $55 \%$ of all responses to reference inquiries were accurate (Hernon \& McClure, 1986). The use of assessment provided evidence that an institution offered students a quality learning experience leading to academic success (Banta, Lund, Black, \& Oblander, 1996; LaPadula, 2003; Ratcliff, 1996). Janes (2002a) received 648 responses from 1,507 libraries surveyed and learned that only 9\% evaluated digital reference services (pp. 551-552). Libraries lacked standard criteria for offering quality digital reference services thereby impeding the ability to determine effective practices.

The researcher asked these questions. Do asynchronous library support services adequately address reference requests made digitally by students? Do librarians respond to students in a timely manner? Do they ask additional or follow-up questions to comprehend fully the student's research request? How do librarians respond to student inquiries? Do they provide students with accurate information? Do they cite the sources used to answer reference questions?

Do these services enhance the student's online learning community? Does the librarian initiate an open and inviting dialogue? Does the librarian attempt to personalize the experience for the student? Does the librarian invite the student to use the service again should additional questions arise? The purpose of the study was to examine the adequacy of asynchronous e-mail reference services offered to DL students at Florida's 28 community college libraries and the contribution of library staff to the students' online learning community. 
To determine the adequacy of asynchronous e-mail reference services offered to DL students at Florida's 28 community colleges and the contribution of library staff to the students' online learning community, the researcher conducted interviews, an unobtrusive study, and a comparative analysis of the unobtrusive study results. The researcher obtained appropriate permission from the administration at IRCC and IRB approval from Nova Southeastern University to conduct the study. Twenty-three DRSPs were interviewed to determine the digital reference policies and procedures in place at their respective institutions and to learn more about their personal opinions regarding the provision of digital reference. An unobtrusive study was conducted by sending 14 questions to the 28 community college libraries using pseudo-names and e-mail accounts established to represent proxies. A comparative analysis was done of the unobtrusive results by reviewing the assessment of the responses by the researcher and by students from IRCC. The researcher selected 48 IRCC student coders from three sources: a pool of students who had already taken or were currently enrolled in DL classes, a pool of students who had already taken or who were currently enrolled in blended classes, and a pool of students who had asked for help from a librarian using e-mail or an online form. These pools helped ensure that the student assessors would have some familiarity with the online environment, but the students' responses were not analyzed based on their respective pools. The researcher used mixed data analysis for quantitative and qualitative data to document the results (Abels, Kaske, \& White, 2002).

The responses were evaluated for accuracy, comprehensiveness, communication technique, timeliness, satisfaction, and qualitative comments made by the researcher and the students. The researcher coded each response using a random number scheme. The 
researcher assessed every reference response. Each student assessed five separate reference responses coded to protect the identity of the DRSP and the DRSP's institution. The two responses were compared using the $t$-Test for Two Independent Samples, with a significance level set at 0.05 and a confidence interval at $95 \%$, to determine if a significant difference existed between the researcher's scores and the students' scores.

The reference queries used in this study reflected questions characteristic of Florida's community college students. The answers to the inquiries were answerable using online resources or standard reference tools since the researcher had no immediate access to the collections at all of Florida's community college libraries. While the answers to the queries did not necessarily have to come from a particular source, the response had to be accurate, the resource had to be current, and the source had to be authoritative. The final list of reference questions comprised actual questions asked of the researcher's "Ask a Librarian" service during the past two years, as well as adaptations of other types of reference questions. Jirjees (1981) said the use of actual questions provided legitimacy despite the use of unobtrusive data gathering.

The researcher submitted the reference questions, the interview questions, and the coding guidelines to a panel of information science experts. None of the panel members were Florida librarians and all agreed to preserve the confidentiality of the study. The panel reviewed the instruments between April 3 and April 20, 2004. They made comments, corrections, and suggestions independent of one another. They reviewed the reference questions for clarity, suitability for the library type, level of difficulty, appropriateness for the typical undergraduate student, and as representative of questions asked at community college libraries. They reviewed the interview questions for clarity, 
suitability for the study topic, appropriateness for the questions raised about asynchronous reference services, and representative of questions asked about reference services. They reviewed the coding guidelines for clarity, suitability for the study topic, appropriateness for an assessment of reference service, and accuracy and consistency in assessment and coding. The panel of experts found the three instruments showed both reliability and content validity. Hernon and McClure (1987a) recommended this approach. The researcher also used inter-rater reliability and found satisfactory agreement within an acceptable percentage range between the researcher and the student assessor. The researcher edited the list based on their remarks and finalized the list of 15 reference questions, 15 interview questions, and the coding guidelines for the study.

The researcher, in the guise of a proxy, sent 14 reference inquiries in an unobtrusive manner to DRSPs within Florida's community college library. Used for the past three decades, unobtrusive testing helped to evaluate the adequacy of reference service, experiences with library staff, and patrons' attitudes toward repeat visits (Baker \& Field, 2000; Hernon \& McClure, 1986, 1987a). This method allowed for the optimum response without triggering the Hawthorne Effect. The Hawthorne Effect indicated that an individual's behavior changes when he or she knows that someone was studying it (Tygett, Lawson, \& Weessies, 1996). The researcher dropped one of the original 15 questions due to technological difficulties. The researcher and Indian River Community College students ( $\mathrm{n}=48$ ) independently evaluated the responses provided to the proxy by the DRSPs. The researcher maintained the unobtrusiveness of the study. The researcher and students assessed the responses submitted by the DRSPs using a coding sheet that 
evaluated the manner in which the DRSP communicated with the proxy, the accuracy of the response, the use of source information, the use of referrals, and satisfaction.

Since library services involved direct interaction with students, the online relationship between the student and the librarian had added challenges. The primary challenge was the absence of visual and verbal clues. Other challenges included the DRSP's inability to assess readily the student's research and technology skills. These challenges, coupled with the lack of standards, exacerbated opportunities to develop an online relationship.

The researcher sent 14 questions to each of the 28 community college libraries for a potential 392 inquiries. The DRSPs answered 240 inquiries for a $61 \%$ return rate. According to Whitlatch (2000), "A response rate of 70 to 80 percent is considered good" (p. 30). Of the 240 responses, the researcher indicated a willingness to return to the same DRSP for assistance $62.5 \%$ of the time while student assessors indicated willingness $58 \%$ of the time. Technology failure accounted for the return of eight possible responses. The researcher aborted the delivery of 12 questions to suspicious DRSPs. Question 4 was dropped from the study due to tremendously slow Web response on the day it was asked. MyDoom worm slowed online access and rendered many outgoing asynchronous requests undeliverable. This slow response time compromised the data collection period.

From these 23 interviews of a possible 28 DRSPs, 17 DRSPs acknowledged not having any policies for digital reference services in place and 18 DRSPs indicated that they did not survey their e-patrons to assess their satisfaction with digital reference. Those libraries assessing their e-patrons used the library's annual survey tool, Web site evaluation tool, course evaluations, library or service satisfaction tool, auto- 
questionnaires, or a survey tool sent immediately after the transaction. More than $50 \%$ of the DRSPs interviewed said they did not monitor digital reference service for quality.

The researcher determined an accurate response with or without source information in $28 \%$ of the responses and a partly accurate response with or without source information in $27 \%$ of the responses. The student assessors determined an accurate response with or without source information in $60 \%$ of the responses and a partly accurate response with or without source information in $26 \%$ of the responses. The researcher indicated source name and source information provided in $71 \%$ of the responses while the student assessors indicated 68\%. Hernon and McClure (1986) stated that librarians typically answered 55\% of questions asked correctly (p. 41).

A difference existed between the researcher's and the students' determination of an accurate response. The researcher possessed a master's degree in library and information studies and 25 years of experience as a research specialist. Student assessors represented the traditional patron. Patrons articulated a higher level of satisfaction with the reference transaction if they obtained part of the information sought (Richardson, 2002). They also expressed appreciation for assistance in conducting research that they could not do for themselves (Quint, 2004). Since patrons did not know if the information was accurate or inaccurate, they assumed that the response given to them was accurate and they rated the transaction accordingly (Richardson). Patrons indicated satisfaction with a useless reply if the reference service provider exhibited respect, helpfulness, and compassion.

The time it took the DRSPs to respond to the questions asked during the unobtrusive part of the study ranged from 6 seconds to 20 days. Ten percent were answered in less than one hour, $17.5 \%$ in one to two hours, $61 \%$ within 24 hours, $75 \%$ within 48 hours, 
$86 \%$ within 72 hours, $90 \%$ within 96 hours, and the remainder took from 5-20 days to answer the question. In terms of the actual response time, DRSPs answered six questions (43\%) within 9-14 hours indicating an above average response time and eight questions (57\%) within 15-24 hours indicating an average response time. In terms of the response time within regular business hours, DRSPs answered two questions (14\%) within 0-3 hours, 11 questions (79\%) within 3-12 hours, and one question (7\%) within 12-36 hours. In terms of communication during the unobtrusive portion of the study, there was no significant difference between the researcher's scores and the students' scores in 12 of the 14 questions. The researcher found that in all 14 questions, some online communication skills were unsatisfactory. The student assessors found in 10 questions that some online communication skills were unsatisfactory, in three questions that online communication skills were adequate, and in one question that the online communication skills were inadequate. Inadequacies appeared in greeting the student, using the student's name, interest, repeating the reference question, asking questions, fully explaining the answer, providing steps for independent research, satisfaction with the answer, invitation to utilize the service again, closing the transaction, and indicating professional position.

With the number of DL programs and courses increasing, standards for and assessment of digital reference services helped to strengthen this support service for students, college administration, and regional accreditors. Since digital reference was still in its early stages, standards guided libraries in the development of new digital references and in the analysis of existing services. Assessment instruments also facilitated the collection of data needed to evaluate digital reference service. The study resulted in recommendations for further study in the areas of the digital environment, unobtrusive 
methodology, standards, accuracy, measurements, online relationships, training of DRSPs and students, institutional responsibility, and promotion. 


\section{Appendix A}

\section{Methodology Workflow}

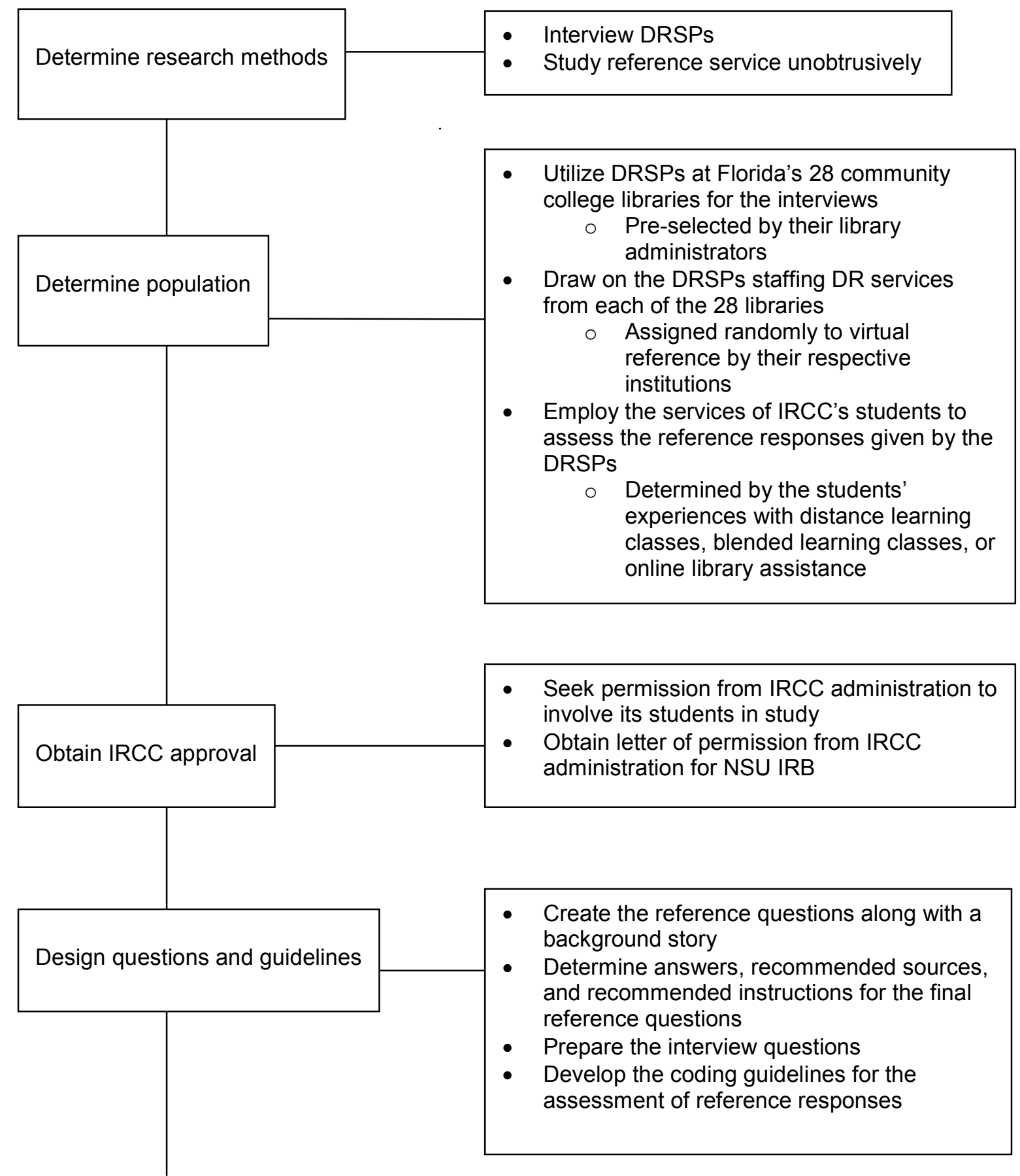




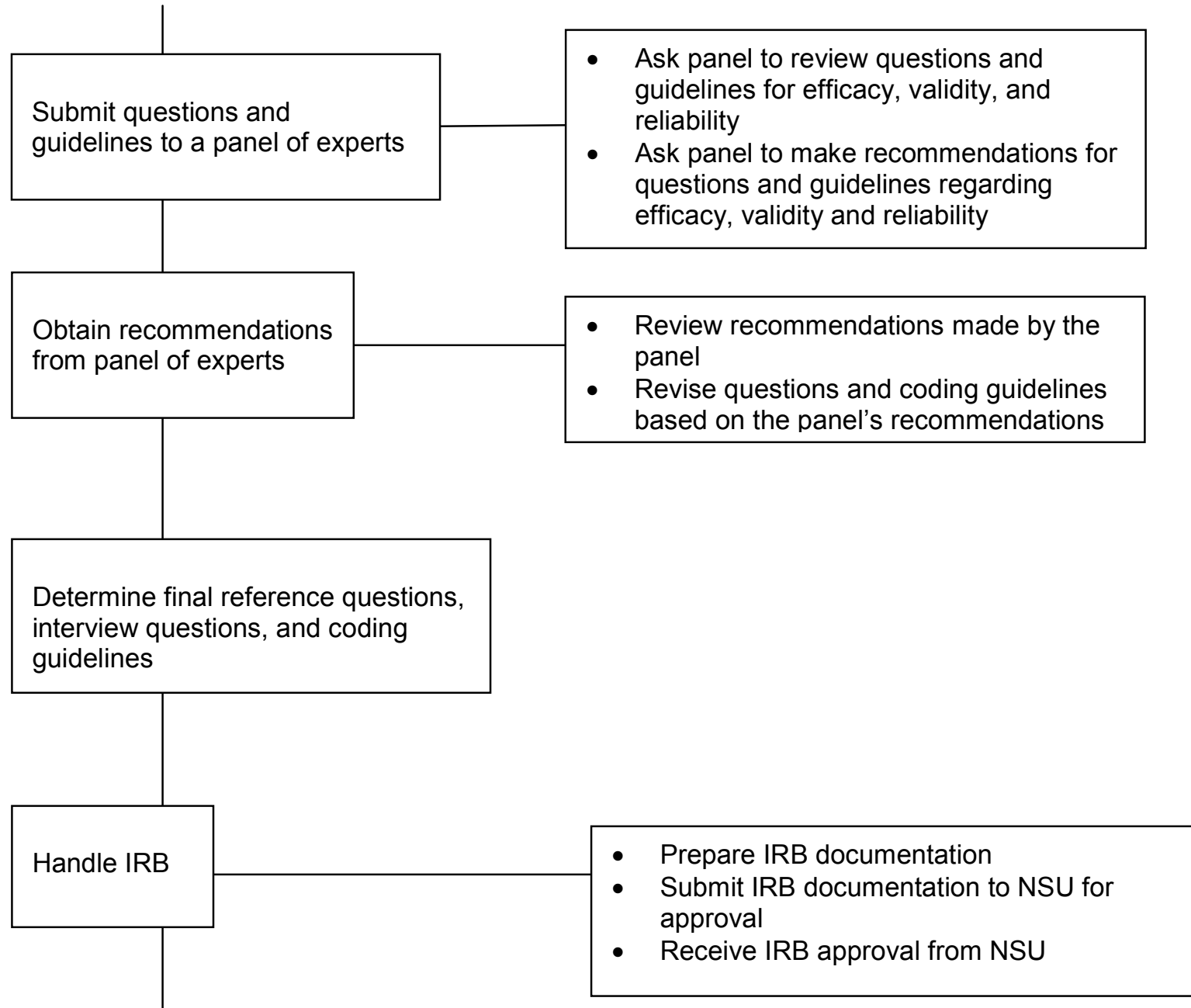

Protect the identity of the DRSPs and their respective institutions

- Use a random number generator to assign a code number to each of the 28 institutions

- Black out any identifying DRSP or institutional names from incoming data

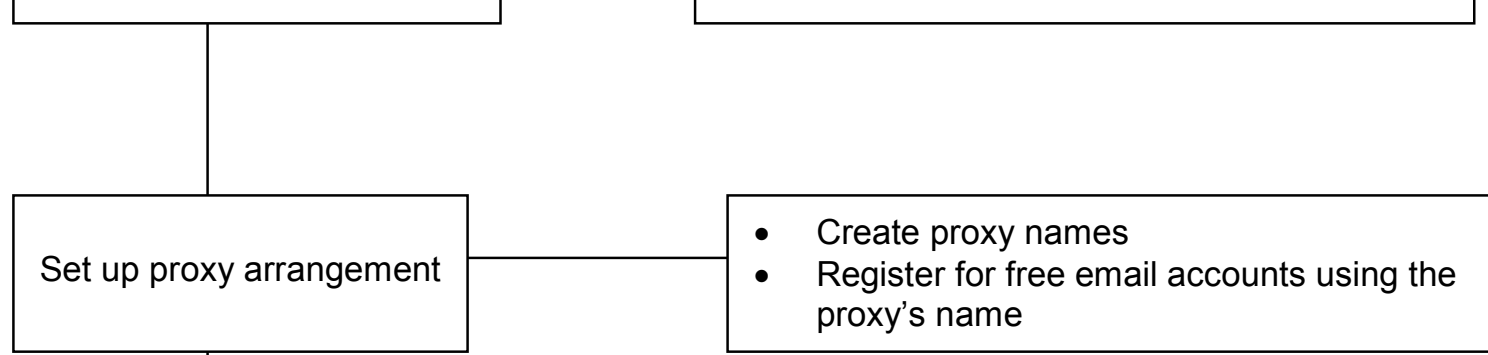




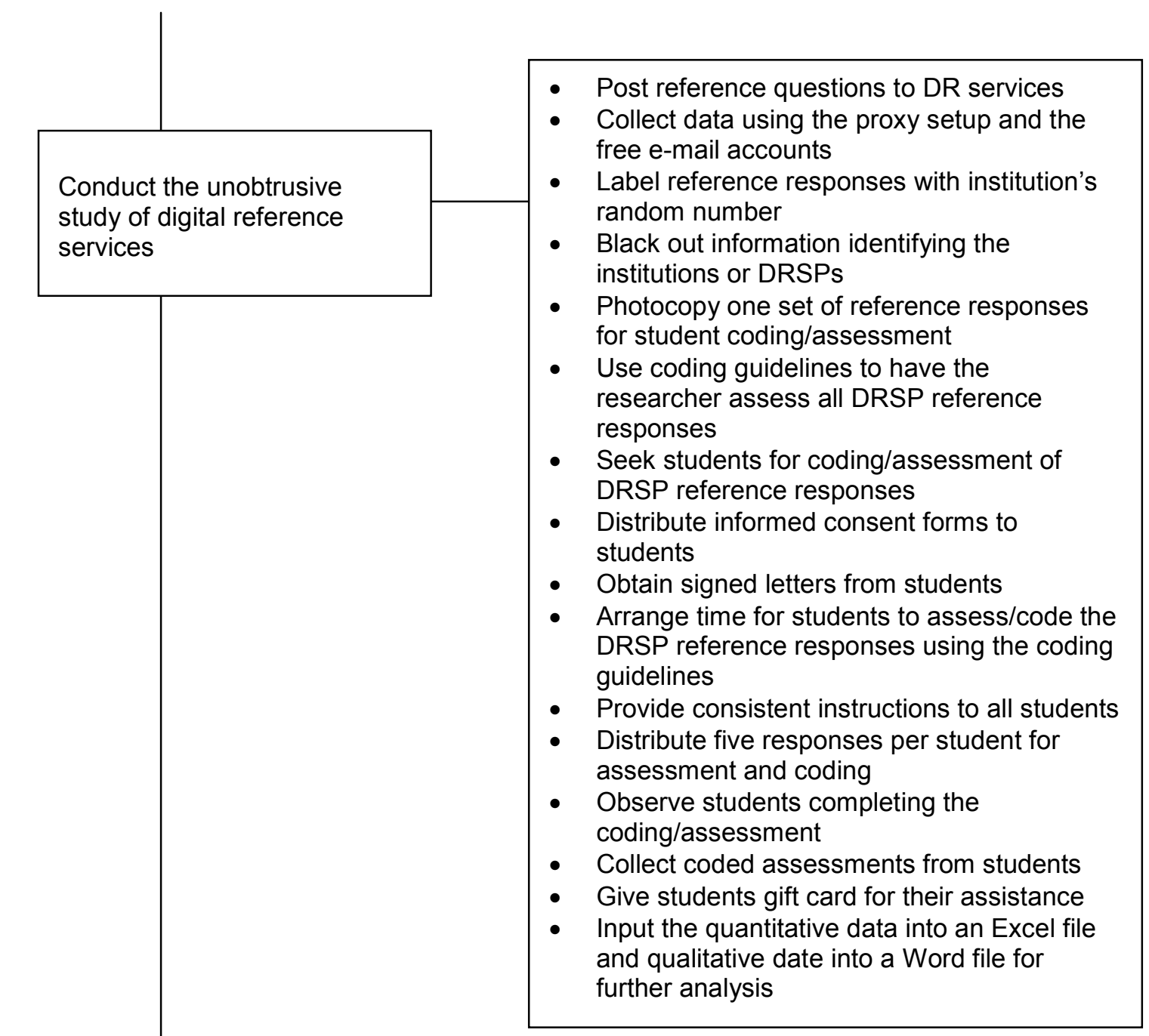

Conduct the interviews of the DRSPs

- $\quad$ Seek permission from 28 library administrators to interview DRSPs

- Receive permission from them

- $\quad$ Contact the DRSPs

- Instruct DRSPs to sign the informed consent forms

- Collect signed letters from them

- Arrange interviews with each DRSP

- Ask interview questions

- Receive interview responses

- Label interview responses with institution's random number

- Input qualitative data into a Word file for further analysis 


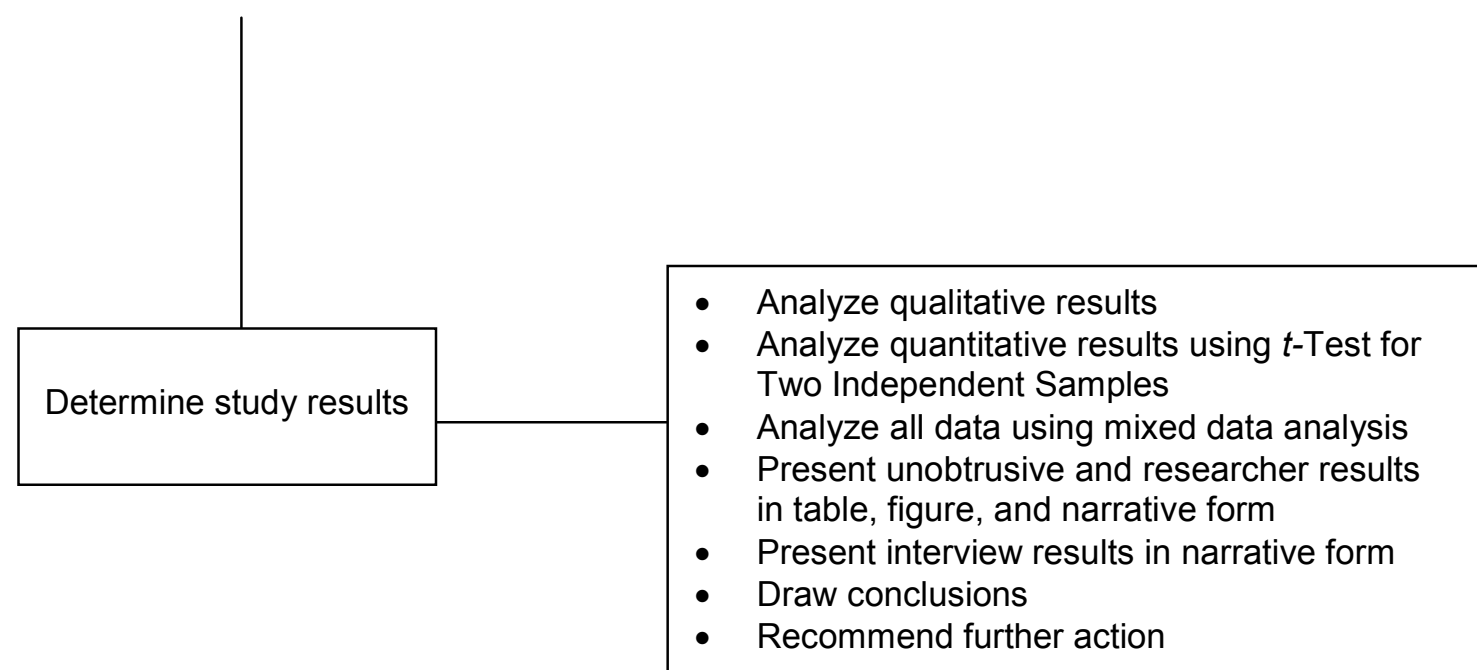




\title{
Appendix B
}

\section{IRB Approval}

Patricia Profeta

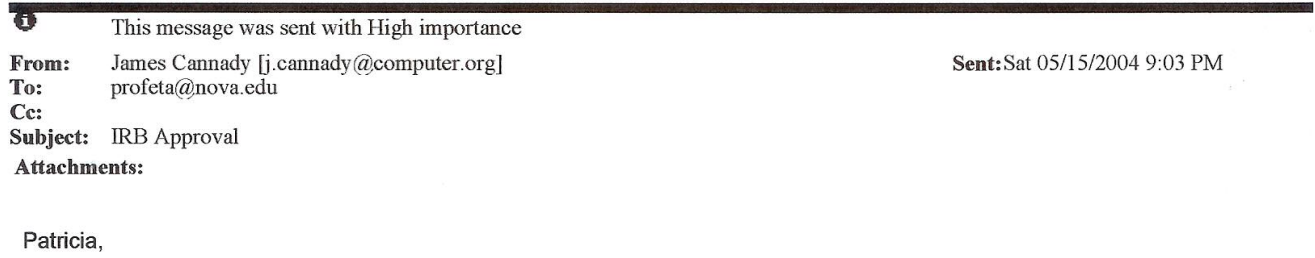

After reviewing your IRB Submission Form and Research Protocol I have approved your proposed research for IRB purposes. Your research has been determined to be exempt from further IRB review based on the following conclusion:

\begin{abstract}
Research using survey procedures or
interview procedures where subjects'

identities are thoroughly protected and

their answers do not subject them to criminal and civil liability.
\end{abstract}

Please note that while your research has been approved, additional IRB reviews of your research will be required if any of the following circumstances occur:

1. If you, during the course of conducting
your research, revise the research

protocol (e.g., making changes to the

informed consent form, survey

instruments used, or number

and nature of subjects).

2. If the portion of your research involving

human subjects exceeds 12 months

in duration.

Please feel free to contact me in the future if you have any questions regarding my evaluation of your research or the IRB process.

Dr. Cannady

James Cannady, Ph.D.

Assistant Professor

Graduate School of Computer

and Information Sciences

Nova Southeastern University

954.262.2085

404.312.2374 (mobile phone)

cannady@nova.edu

PGP public key fingerprint:

8169 6D03 680E EF6C $899 \mathrm{C}$

8 C42 B4A3 DC9F 9F6B 4075 


\section{Appendix C}

\section{Letter of Permission from IRCC Administrator}

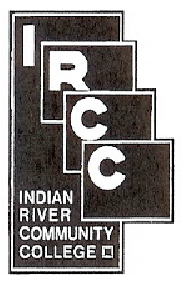

\section{INDIAN RIVER COMMUNITY COLLEGE \\ 3209 VIRGINIA AVENUE • FORT PIERCE, FLORIDA 34981-5596 \\ TELEPHONE 772-462-4704 • SUNCOM 246-4704 • FAX 772-467-7804 or 772-467-8004 EMAIL: smaxwell@ircc.edu}

May 3, 2004

Institutional Review Board

Office of Grants and Contracts

Nova Southeastern University

3301 College Avenue

Fort Lauderdale, Florida 33314

Dear Review Board,

Ms. Pat Profeta has discussed with me, her intentions of conducting research concerning the adequacy of asynchronous e-mail reference service offered to distance learning students at Florida's 28 community college libraries and the affect on-line communication, by library staff, has on the students' on-line learning community.

Ms. Profeta's professional responsibilities at Indian River Community College include overall supervision of library services. As such, the research she proposes will be valuable to strengthening those services. Some of the students in her study will be students of Indian River Community College.

I wholeheartedly approve of this research and of her continuing efforts to provide the best possible library services to all students including distance learning students.

\section{Sincerely,}

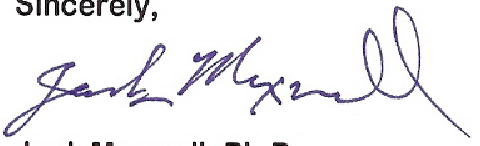

Jack Maxwell, Ph.D.

Vice President of Academic Affairs

. IM/alh 


\section{Appendix D}

\section{Permission from Library Administrators}

The following is an explanation of the project that library administrators will receive from the principal investigator to authorize participation of their respective digital reference service providers in the interviews. The principal investigator will contact the administrators via personal e-mail, the library administrators' listserv, or by telephone. The library administrators within Florida's 28 community college libraries participate in a restricted listserv. The principal investigator is also a library administrator within the Florida community college system.

I am working on my doctoral dissertation addressing the effectiveness of asynchronous reference service for distance learning students. I am seeking permission to interview one of your digital reference staff. The interview will take approximately 30 minutes and the questions ask for their observations about asynchronous e-mail reference service offered currently to students at your institution. Responses will be reported by the state as a whole and by the three institution size groups, small, medium, and large. If you agree to allow your digital reference staff to participate, please identify the individual you consider most experienced with your library's digital reference service. Your e-mailed response indicates your consent. Your employee also has the right to decline participation.

All information obtained in this study related to the participant's and the institution's identity is strictly confidential unless disclosure is required by law. The use of unique random numbers, the removal of identifying data, and the security of original data allows for the anonymity of participants and institutions. I have a secure location for all data collected.

The Internet introduced significant changes in academic, business, and home environments. A growing number of college students now take classes through the Internet. Distance learning students often require library support services, i.e., digital reference service, to complete their coursework. This study involves digital reference service, which provides mediated assistance to students within the online environment. The purpose of the study is to focus on developing standards for digital reference service. The study may contribute to the growing research addressing the adequacy of library support services for distance learning students, particularly in the area of digital 
reference, asynchronous communication, and the impact asynchronous support has on the online learning community of distance learning students.

Thanks for your support.

Pat

Patricia C. Profeta

Assistant Dean of Learning Resources

Indian River Community College

Fort Pierce, FL

Ph.D. student at Nova Southeastern University

Advisor

Dr. Steven D. Zink

Vice President, Information Technology \& Dean, University Libraries

University of Nevada, Reno

Reno, NV 89557

(775) 784-6500, ext. 252 


\section{Appendix E}

\section{Institutional Review Board for Research with Human Subjects (IRB)}

\section{Adult/General Informed Consent [DRSP]}

Funding Source: None

Principal Investigator

Patricia C. Profeta

$35503^{\text {rd }}$ Place SW

Vero Beach, FL 32968

pprofeta@ircc.edu

(772) 462-4479

\author{
Advisor \\ Dr. Steven D. Zink \\ Vice President, Information Technology \\ Dean, University Libraries \\ University of Nevada, Reno \\ IT/Library Administrative Offices \\ 1664 North Virginia Street \\ Reno, NV 89557 \\ (775) 784-6500, ext. 252
}

Institutional Review Board

Office of Grants and Contracts

Nova Southeastern University

(954) 262-5369

Effectiveness of Asynchronous Reference service for Distance Learning Students

Description of the Study:

The Internet introduced significant changes in academic, business, and home environments. A growing number of college students now take classes through the Internet. Distance learning students often require library support services, i.e., digital reference service, to complete their coursework. This study involves digital reference service, which provides mediated assistance to students within the online environment. The purpose of the study is to focus on developing standards for digital reference service. The study may contribute to the growing research addressing the adequacy of library support services for distance learning students, particularly in the area of digital reference, asynchronous communication, and the impact asynchronous support has on the online learning community of distance learning students. 
Digital reference librarians from Florida's 28 community college libraries are being asked to participate in an interview lasting approximately 30 minutes asking for their observations about asynchronous e-mail reference service being offered currently to students at their respective institutions. Responses will be reported by the state as a whole and by the three institution size groups, small, medium, and large.

The risk to participants is negligible, as the principal investigator has a method to insure protection of the participant's identity. All information obtained in this study related to the participant's identity is strictly confidential unless disclosure is required by law. The use of unique random numbers, the removal of identifying data, and the security of original data allows for the anonymity of participants. The principal investigator has a secure location for data collected. The benefit to participants is the use of published results for use within each respective institution's digital reference department.

There are no costs to or payments made to any interviewees.

Participation is voluntary, and refusal to participate carries no penalty. Participants are free to ask questions at any point and they are free to withdraw from participation at any time without penalty. If a participant chooses to withdraw, he/she may request that the principal investigator destroy any data collected from him/her unless prohibited by state or federal law.

If significant new information relating to the study becomes available which may relate to each participant's willingness to continue to participate, the principal investigator will provide this information to the participants.

The participant may contact the principal investigator with questions. Contact information is located at the top of the consent form.

Voluntary Consent by Participant:

I have read the preceding consent form, or it has been read to me, and I fully understand the contents of this document and voluntarily consent to participate. All of my questions concerning the research have been answered. I hereby agree to participate in this research study. If I have any questions in the future about this study, they will be answered by Patricia C. Profeta. A copy of this form has been given to me. This consent ends at the conclusion of this study.

Participant's Signature:

Date:

Participant's Name (printed): 


\section{Appendix F}

\section{Checklist for Quality E-mail Reference Responses \\ Check only those categories you believe the response covers. \\ I. Communication between library employee and student}

_Employee offers a friendly or welcoming greeting to student.

_Employee uses the student's name or e-mail address as part of the greeting.

_Employee shows interest in the student's question (comment offered).

_ Employee does NOT criticize the student's question.

_Employee repeats the question to clarify the student's question.

_Employee asks questions to get more information from the student.

_Employee explains what he/she did to answer the student's question.

_Employee provides instructions so the student can duplicate the steps.

__Spelling/grammatical errors DO NOT appear in the employee's response.

_Employee writes a clear and understandable response.

_Employee asks if he/she has answered the student's question.

_Employee invites the student to contact or use the service again.

_Employee includes a closing message.

_Employee signs his/her name.

_Employee indicates his/her library position or title. 


\section{Time taken to answer student's question}

_ hour(s) __ minute(s) Time employee takes to respond to student's request.

The elapsed time included a weekend. _ Yes _ No

The elapsed time included a night. _ Yes _ No

Student contacted if response took more than 24 hours. _ Yes _ No

\section{Responses given to the student:}

\section{a) Accuracy of the employee's answer}

Answer is accurate and includes source information.

_. Answer is accurate, but lacks source information.

Answer is partly accurate and includes source information.

Answer is partly accurate, but lacks source information.

Answer is inaccurate or incorrect and includes source information.

Answer is inaccurate or incorrect, but lacks source information.

Answer includes more than one source.

Answer indicates "don't know" type of response.

b) Employee includes a referral in the response

Provides part/all of the answer and still suggests a referral.

__Provides no answer, but still suggests a referral.

_Provides no answer and provides NO referral.

c) Employee's answer and referral to another person, resource, or service

_ Refers student to a source and source information.

_Refers student to a source, but provides no source details. 
Refers student to another person. Employee acts as a go-between for the student.

Refers student to another person. Employee provides no additional assistance.

Refers student to another library service. Employee acts as a gobetween for the student.

Refers student to another library service. Employee provides no additional assistance.

\section{Student Rater's Response}

Would you ask this library employee for help? _ Yes _ No Comments: 


\section{Appendix G}

\section{Scoring Quality E-mail Reference Responses}

I. Communication between library employee and student

If the response addresses the category, give that category one (1) point.

15 points $=$ Excellent online communication skills

10-14 points $=$ Adequate online communication skills

6-9 points $=$ Some online communication skills unsatisfactory

$0-5$ points $=$ Online communication skills inadequate

II. Time taken to answer student's question

Subtract weekend hours between 5pm on Friday through 8am on Monday. Subtract weekday evening hours between 10pm and 8am.

15 points $=0-3$ hours

14 points $=>3-6$ hours

13 points $=>6-9$ hours

12 points $=>9-12$ hours

11 points $=>12-15$ hours

10 points $=>15-18$ hours

9 points $=>18-24$ hours

8 points $=>24-36$ hours

7 points $=>36-48$ hours

6 points $=>48-72$ hours

5 points $=>72$ hours

0 points $=$ Not answered

15 points $=$ Excellent response time

12-14 points $=$ Above average response time

8-11 points $=$ Average response time*

5-7 points $=$ Below average response time*

0 points $=$ Inadequate and unacceptable*

*Review digital reference processes for ways to improve turn-around time. 
III. Responses give to the student

a. Accuracy of the employee's answer

3 points $=$ Accurate answer and source information

2 points $=$ Accurate answer without source information

1 point $=$ Partly accurate with or without source information

1 point $=$ Inaccurate with or without source information

0 points $=$ Don't know answer

Add 1 point for extra, but accurate source(s)

b. Employee includes a referral in the response

2 points $=$ Referral not needed

2 points $=$ Part/all answer and referral

1 point $=$ No answer with referral

0 points $=$ No answer and no referral

c. Employee's answer and referral to another person, resource, or service

2 points $=$ Referral not needed

2 points $=$ Referral with source, person, or service information/facilitation

1 point $=$ Referral without source, person, or service information/facilitation

0 points $=$ No answer

IV. Student Rater's Response

2 points $=$ Yes

1 point $=$ No

Quality of Sections III and IV

10 points $=$ Excellent response - Accurate, documented, and effective

9 points $=$ Average response - Accurate and effective

8 points $=$ Needs improvement in accuracy category, but has redeeming features

7 points $=$ Needs improvement in accuracy category

3-6 points $=$ Needs improvement in accuracy category and less reliance on referrals

$0-2$ points $=$ Inadequate assistance provided to student 


\title{
Appendix $\mathrm{H}$
}

\author{
Expert Panel
}

Panel Member

- Professional Expertise

○ Computer and Information Science professional, 18 years

- Current Position

- Software Developer, private company

- Graduate Degrees and Certificates

- Master of Science in Library Science

○ Doctor of Philosophy in Information Science, ABD

Panel Member

- Professional Expertise

- Library and Information Science professional, 5 years

- Academic Librarian, 5 years

- Current Position

- Academic Librarian, university library

- Graduate Degrees and Certificates

○ Master of Library and Information Science

Panel Member

- Professional Expertise

- Library and Information Science professional, 17 years

- Special Librarian, 10 years

- Records and Information Manager, 7 years

- Adjunct Instructor (university) - traditional and distance learning graduate courses, 7 years

- Current Position

○ Records Manager with special library function, state agency

- Graduate Degrees and Certificates

○ Master of Library and Information Science

- Certified Records Manager, Institute of Certified Records Managers

○ Doctor of Philosophy in Information Science, ABD 
Panel Member

- Professional Expertise

- Computer and Information Science professional, 22 years

- Information Architect - specialties include user-centered analysis and design using quantitative and qualitative methods such as surveys, focus groups, field studies informal user validation, heuristic evaluation, and formal usability testing, 22 years

- Instructional Designer - specialties include design and development of interactive training and performance support systems delivered in electronic environments, 22 years

- Consultant for Fortune 1000 clients, 22 years

$\circ$ Manager overseeing project and development teams, 14 years

- Current Position

○ Director of Information Architecture, private company

- Graduate Degrees and Certificates

- Master of Arts in Instructional Design

○ Doctor of Philosophy in Information Science, ABD

Panel Member

- Professional Expertise

- Computer, Library, and Information Science professional, 32 years

- Computer Software Analyst, 25 years

- Software Research and Developer, 18 years

- Academic Librarian, 7 years

- Current Position

- Chief Scientist, private company

- Graduate Degrees and Certificates

- Master of Library and Information Science

- Master of Administrative Science

○ Doctor of Philosophy in Information Science, ABD

Panel Member

- Professional Expertise

- Academic Librarian, 25 years

- Academic Library Administrator, 20 years

- Assistant Professor (university) - traditional and distance learning undergraduate courses, 25 years (traditional) and 10 years (distance learning)

- Current Position

- Assistant Director, university library

- Assistant Professor, university

- Graduate Degrees and Certificates

- Master of Library and Information Science

- Doctor of Philosophy in Information Science, ABD 


\title{
Appendix I
}

\section{Institutional Review Board for Research with Human Subjects (IRB)}

\author{
Adult/General Informed Consent [student]
}

Funding Source: None

IRB approval \#

Principal Investigator

Patricia C. Profeta

$35503^{\text {rd }}$ Place SW

Vero Beach, FL 32968

pprofeta@ircc.edu

(772) $462-4479$

Institutional Review Board

Office of Grants and Contracts

Nova Southeastern University

(954) 262-5369
Advisor

Dr. Steven D. Zink

Vice President, Information Technology

Dean, University Libraries

University of Nevada, Reno

1664 North Virginia Street

Reno, NV 89557

(775) 784-6500, ext. 252

Effectiveness of Asynchronous Reference service for Distance Learning Students

Description of the Study:

The Internet introduced significant changes in academic, business, and home environments. A growing number of college students now take classes through the Internet. Distance learning students often require library support services, i.e., digital reference service, to complete their coursework. This study involves digital reference service, which provides mediated assistance to students within the online environment. The purpose of the study is to focus on developing standards for digital reference service. The study may contribute to the growing research addressing the adequacy of library support services for distance learning students, particularly in the area of digital reference, asynchronous communication, and the impact asynchronous support has on the online learning community of distance learning students. 
Students at Indian River Community College who are over the age of 18 years are being asked to assess the reference responses provided to the principal investigator, Patricia C. Profeta.

The risk to participants is negligible, as a method to insure protection of the participant's identity has been established. The collection of data ensures the participant's protection using unique and non-repeatable random numbers, as well as the maintenance of data in secure files. The benefit to students from assessing digital reference responses is to gain a better understanding of digital reference service within the online environment.

There are no costs to the students. Payments of $\$ 10$ will be made to those students who assess completely five digital reference responses. The five assessments should not take more than 30 minutes.

All information obtained in this study related to the participant's identity is strictly confidential unless disclosure is required by law. The use of unique random numbers, the removal of identifying data, and the security of original data allows for the anonymity of participants.

Participation is voluntary, and refusal to participate carries no penalty. Participants are free to ask questions at any point and they are free to withdraw from participation at any time without penalty. If a participant chooses to withdraw, the principal investigator cannot pay the $\$ 10$ to the participant. If a participant chooses to withdraw, he/she may request that the principal investigator destroy any data collected from him/her unless prohibited by state or federal law.

If significant new information relating to the study becomes available which may relate to each participant's willingness to continue to participate, the principal investigator will provide this information to the participants.

The participant may contact the principal investigator with questions. Contact information is located at the top of the consent form.

Voluntary Consent by Participant:

I have read the preceding consent form, or it has been read to me, and I fully understand the contents of this document and voluntarily consent to participate. All of my questions concerning the research have been answered. I hereby agree to participate in this research study. If I have any questions in the future about this study, they will be answered by Patricia C. Profeta. A copy of this form has been given to me. This consent ends at the conclusion of this study.

Participant's Signature:

Date:

Participant's Name (Printed): 
As a token of my appreciation for your taking the time to assist with the coding of five digital reference responses, I would you to select one of the following gifts:

$\square \quad \$ 10$ gift certificate for Target

$\$ 10$ gift certificate for the IRCC Pioneer Bookstore

$\$ 10$ gift certificate for Wal-Mart 


\section{Reference List}

Abels, E. G. (1996). The e-mail reference interview. RQ, 35, 345-358.

Abels, E. G. (2000, June). Remote electronic reference. Panel presentation conducted at the Special Libraries Association Annual Conference, Philadelphia, PA. Retrieved October 6, 2003, from http://www.library.northwestern.edu/transportation/slatran/ philpresents/ReferenceLinks.htm

Abels, E. G., Kaske, N., \& White, M. D. (2002, November). Evaluation of chat-based reference service. Paper presented at the Virtual Reference Desk Conference, Chicago, IL. Retrieved January 17, 2004, from http://www.vrd.org/conferences/ VRD2002/proceedings/abels.shtml

Abels, E. G., \& Liebscher, P. (1994). A new challenge for intermediary-client communication: The electronic network. Reference Librarian, (41/42), 185-196.

Abels, E. G., \& Ruffner, M. (2004, November). Online virtual reference training. In Proceedings of the Virtual Reference Desk Conference 2004, Cincinnati, OH. Retrieved October 8, 2005, from http://www.vrd2004.org/proceedings/presentation. cfm? PID=341

Agre, P. (1994). Art of getting help. Network Observer, 1(2). Retrieved October 6, 2003, from http://dlis.gseis.ucla.edu/people/pagre/getting-help.html

Aluri, R. (1993). Improving reference service: The case for using a continuous improvement method. $R Q, 33,220-236$.

American Library Association, Association of College and Research Libraries. (2004a). Guidelines for distance learning library services. Retrieved March 12, 2005, from http://www.ala.org/ala/acrl/acrlstandards/guidelinesdistancelearning.htm

American Library Association, Board on the Library and Adult Education, \& National University Extension Association, Committee on Library Cooperation. (1931). Books for the extension student. Bulletin of the American Library Association, 30, 674-687.

American Library Association, Management of Reference Services Committee, Management and Operation of Public Services Section, Reference and Adult Services Division. (1994). Information services policy manual: An outline. (1994). RQ, 34, 165-172. 
American Library Association, Reference and User Services Association, Management of Reference Committee. (2004b). Guidelines for behavioral performance of reference and information services professionals. Retrieved July 26, 2004, from http://www.ala.org/ala/rusa/rusaprotools/referenceguide/guidelinesbehavioral.htm

American Library Association, Reference and User Services Association, MachineAssisted Reference Section. (2004c). Guidelines for implementing and maintaining virtual reference services. Retrieved July 26, 2004, from http://www.ala.org/ala/rusa/ rusaprotools/referenceguide/virtrefguidelines.htm

American Library Association, Reference and User Services Association, Task Force on Professional Competencies. (2003). Professional competencies for reference and user services librarians. Retrieved July 26, 2004, from http://www.ala.org/ala/rusa/ rusaprotools/referenceguide/professional.htm

Ammentorp, S., \& Hummelshoj, M. (2001, May/June). Ask a librarian: Web-based reference question services: A model for development. Paper presented at the Nordic Conference on Information and Documentation, Reykjavik, Iceland. Retrieved November 30, 2003, from http://www.bokis.is/iod2001/papers/Ammentorp_paper.doc

Anderson, C. R. (2003). Puzzles and essays from "The Exchange": Tricky reference questions. New York: Haworth Press.

Anderson, E., Boyer, J., \& Ciccone, K. (2000, October). Remote reference services at the North Carolina State University libraries. Paper presented at the Virtual Reference Desk Conference, Seattle, WA. Retrieved January 17, 2004, from http://www.vrd.org/conferences/VRD2000/proceedings/boyer-anderson-ciccone1214.shtml

Archer, S. B., \& Cast, M. (1999). "Going Where the Questions Are": Using media to maintain personalized contact in reference service in medium-sized academic libraries. Reference Librarian, 31(65), 39-50.

Armstrong, D. (2003, September 17). Distance learning is the way of the future Accessible and efficient. Community College Newsletter, (2003-10), 4-5.

Arnold, J., \& Kaske, N. (2002). Chat with a librarian. Retrieved January 17, 2004, from http://www.lib.umd.edu/groups/digref/cil/ppt

AskERIC error page. (2003). Retrieved January 10, 2004, from http://www.askeric.org/ cgi-bin/res.cgi/Reference

Association of Research Libraries. (2002). Reference service statistics and assessment: Executive summary. Retrieved September 14, 2003, from http://www.arl.org/spec/ 268sum.html 
Association of Research Libraries. (1999). Trends in ARL libraries: An introduction to ARL statistics, 1998-1999. Retrieved February 1, 2004, from http://www.arl.org/stats/ arlstat/99intro.html

Association of Southeastern Research Libraries. (2003). ASERL virtual reference membership survey complete results (preliminary results). Retrieved January 3, 2004, from http://aserl.solinet.net/projects/vref/surveysumcomplete.htm

Astin, A. W. (1993). What matters in college? Four critical years revisited. San Francisco: Jossey-Bass.

Ault, M., \& Viggiano, R. (2000). Going the distance: Traditional reference services for non-traditional users. Florida Libraries, 43(2), 6-7.

Baker, L. M., \& Field, J. J. (2000). Reference success: What has changed over the past ten years? Public Libraries, 39, 23-27, 30.

Baker, S. L., \& Lancaster, F. W. (1991). Measurement and evaluation of library services (2nd ed.). Arlington, VA: Information Resources Press.

Banta, T. W., Lund, J. P., Black, K. E., \& Oblander, F. W. (1996). Assessment in practice. San Francisco: Jossey-Bass.

Bao, X. (2003). A study of web-based interactive reference services via academic library home pages. Reference \& User Services Quarterly, 42, 250-256.

Barcellos, S. (2000, October). Understanding intermediation in a digital environment: An exploratory case study. Paper presented at the Virtual Reference Desk Annual Conference, Seattle, WA. Retrieved October 31, 2003, from http://www.vrd.org/ conferences/VRD2000/proceedings/Barcellos_final.shtml

Bargellini, M. L., \& Bordoni, L. (2001). The role of the library in a new learning scenario. Electronic Library, 19, 153-157.

Barkley, D. C. (1998). Public service guidelines in an electronic environment in federal depository libraries. Government Information Quarterly, 15, 73-85.

Baron, N. (1998). Letters by phone or speech by any other means: The linguistics of e-mail. Language and Communication, 18, 133-170.

Barsun, R. (2000). Computer mediated conferencing-mail, telephone: A holistic approach to meeting students' needs. In P. S. Thomas (Ed.), The Ninth Off-Campus Library Services Conference proceedings, Portland, OR (pp. 19-27). Mount Pleasant, MI: Central Michigan University. 
Barsun, R. (2002, April). It's my library, too, Isn't it? In P. B. Mahoney (Ed.), The Tenth Off-Campus Library Services Conference proceedings, Cincinnati, OH (pp. 41-60). Mount Pleasant, MI: Central Michigan University.

Bauman, P. (2002, August 15). Student retention: What you can control, and how. Distance Education Report, 6(16), 8.

Beach Safety, Florida HB 1395, Regular Session (2005).

Beagle, D. (2000). Web-based learning environments: Do libraries matter? College \& Research Libraries, 61, 367-379.

Becker, K. A. (1994). Corralling "creeping featurism": Nurturing a more human-centered technology. Database, 17(2), 8-9. Retrieved June 26, 2004, from ACM Digital Library database.

Bell, J. G., \& Levy, A. P. (2004). Making the digital connection more personal. In R. D. Lankes, J. Janes, L. C. Smith, \& C. M. Finneran (Eds.), Virtual reference experience: Integrating theory into practice (pp. 139-161). New York: Neal-Schuman.

Bichelmeyer, B. A., \& Kiggins, E. A. (1998). Using Web-based conferencing in postsecondary instruction. In Distance Learning '98: Proceedings of the Annual Conference on Distance Teaching and Learning (pp. 67-72). (ERIC Document Reproduction Service No. ED422845)

Blenkinsopp, H. (1992). How's the water?: The training of reference librarians. The Reference Librarian, (38), 175-182.

Bober, M. J., \& Dennen, V. P. (2001). Intersubjectivity: Facilitating knowledge construction in online environments. Educational Media International, 38, 241-250.

Bonham, M. (1987). Library services through electronic mail. College \& Research Libraries News, 48, 537-538.

Borgman, C. L. (1996). Why are online catalogs still hard to use? Journal of the American Society for Information Science, 47, 387-400.

Bourne, C. P. (1965). Some user requirements stated quantitatively in terms of the 90 percent library. In A. Kent \& O. E. Taulbee (Eds.), Electronic information handling (pp. 93-110). Washington: Spartan.

Bristow, A. (1992). Academic reference service over electronic mail. College \& Research Libraries, 53, 631-637.

Bristow, A., \& Buechley, M. (1995). Academic reference service over e-mail: An update. College \& Research Libraries News, 56, 459-462. 
Bristow, A., \& Liu, J. (1999). Academic reference service in the web environment. Retrieved October 31, 2003, from http://www.indiana.edu/librcsd/reference/email/03.html

Brownlee, D., \& Ebbers, F. (2002, April). Separate but unequal? Do Web-based services fulfill their promises? In P. B. Mahoney (Ed.), The Tenth Off-Campus Library Services Conference proceedings, Cincinnati, OH (pp. 61-67). Mount Pleasant, MI: Central Michigan University.

Buehler, M. A. (2004). Where is the library in course management software? Journal of Library Administration, 41(1/2), 75-84.

Bundy, A. (2000, October). Best value: Libraries. Paper presented at the Australian Library and Information Association Conference, Canberra, Australia. Retrieved March 13, 2005, from http://www.library.unisa.edu.au/papers/value.htm

Bunge, C. A. (1990). Factors related to output measures for reference services in public libraries: Data from thirty-six libraries. Public Libraries, 29, 42-47.

Bunge, C. A. (1999). Gathering and using patron and librarian perceptions of questionanswering success. Reference Librarian, 31(66), 115-140.

Burek-Pierce, J. (2002). Digital discomfort? "Get over it," says McClure. American Libraries, 33(5), 45.

Burge, E. J. (2001). Behind the screen thinking: Key factors for librarianship in distance education. In P. Brophy, S. Fisher, \& Z. Clarke (Eds.), Libraries without walls 4: The delivery of library services to distant users (pp. 7-15). London: Facet Publishing.

Burge, E. J. (1994). Learning in computer conferenced contexts: The learners' perspective. Journal of Distance Education, 9(1), 19-43.

Burgin, R., \& Hansel, P. (1990). Reference accuracy: Improving our chances. Wilson Library Bulletin, 65(1), 66-68.

Burke, J. J. (1996). Using e-mail to teach: Expanding the reach of BI. Research Strategies, 14, 36-43.

Burton, P. (1990). Accuracy of information provision: The need for client-centered service. Journal of Academic Librarianship, 22(4), 201-215.

Bushallow-Wilbur, L., DeVinney, G., \& Whitcomb, F. (1996). Electronic mail reference service: A study. $R Q, 35,359-371$. 
Butkovich, N. J. (1988, May). Implementing an expert system for use by undergraduates. In M. E. Williams \& T. H. Hogan (Eds.), Proceedings of the Ninth National Online Meeting, New York, NY (pp. 45-47). Medford, NJ: Learned Information.

Cain, D. L., \& Lockee, B. (2002). Student support services at a distance: Are institutions meeting the needs of distance learners? (ERIC Document Reproduction Service No. ED468729)

Campbell, J. D. (1999). Clinging to traditional reference services: An open invitation to Libref.com. Reference \& User Services Quarterly, 39, 223-227.

Cannon, N. (2002). Yahoo! Do you Google? Virtual reference overview. Reference Librarian, 37(77), 31-37.

Canter, D., Coffey, T., Huntley, M., \& Missen, C. (2000). Predicting serial killers' home base using a decision support system. Journal of Quantitative Criminology, 16, 457-478. Retrieved date, from Academic Search Premier database.

Carlson, S. (2002, July 19). Survey finds that students use the Web but recognize its limitations. Chronicle of Higher Education. Retrieved December 13, 2003, from http://chronicle.com/free/2002/07/2002071901t.htm

Carlson, S. (2004, November 15). Online-education survey finds boom in enrollment and broad satisfaction with courses. Chronicle of Higher Education, 1-2. Retrieved November 15, 2004, from http://chronicle.com/daily/2004/11/2004111503n.htm

Carr, D. (1986). The meanings of the adult independent library learning project. Library Trends, 35, 327-345.

Carter, D. S., \& Janes, J. (2000). Unobtrusive data analysis of digital reference questions and service at the Internet Public Library: An exploratory study. Library Trends, $49,251-265$.

Casado, M. (2001, April). Delivering library services to remote users. Computers in Libraries, 21(4), 32-38.

Casey, A. M., Sochrin, S., \& Race, S. F. (2002). Fair is fair, or is it? Library services to distance learners. Journal of Library Administration, 37, 147-161.

Caspars, J. S., \& Ragan, L. C. (2000). The evolution of distance learning environments: Shift happens. Serials Librarian, 38, 123-133.

Cegles, K. A. (1998). Emerging issues affecting distance education research and practice in higher education: A global futures perspective. Ann Arbor, MI: UMI Publications. 
Chakraborty, M., \& Tuñón, J. (2002). Taking the distance out of library services offered to international graduate students: Considerations, challenges, and concerns. Journal of Library Administration, 37, 163-176.

Chandler, Y. J. (2001). Reference in library and information science education. Library Trends, 50, 245-263.

Cheng, R. J., Nathanson, A. J., MacFarlane, H., Hansen, B., \& Berger, S. (2002). Collaborative digital reference in small academic liberal arts college libraries: Connecticut College, Smith College, and Wesleyan University, 2001-2002. Resource Sharing \& Information Networks, 16, 169-188.

Childers, T. (1980). The test of reference. Library Journal, 105, 924-928.

Christensen, C. M. (1997). The innovator's dilemma: when new technologies cause great firms to fail. Boston: Harvard Business School Press.

Clarke, Z., \& Brinkley, M. (2000). Management information for the delivery of high quality services to distant users in the hybrid library environment. In P. Brophy, S. Fisher, \& Z. Clarke (Eds.), Libraries without walls 3: The delivery of library services to distant users (pp. 266-275). London: Library Association Publishing.

Cocheo, S., Harris, K., \& Kirk, A. (2003). Community bank competitiveness survey. ABA Banking Journal, 95(2), 40.

Coffman, S. (2002). Be it resolved that reference librarians are not toast. American Libraries, 33(3), 51-54.

Coffman, S. (2004, September). To chat or not to chat - Taking yet another look at virtual reference, part 2. Searcher, 12(8), 49-57. Retrieved September 1, 2004, from http://www.infotoday.com/searcher/sep04/arret_coffman.shtml

Coffman, S., \& McGlamery, S. (2000). The librarian and Ask Jeeves. American Libraries, 31(5), 66.

Coffman, S., \& Saxton, M. L. (1999). Staffing the reference desk in the largely-digital library. Reference Librarian, 31(66), 141-163.

College Center for Library Automation. (2003a, September 11). The College Center for Library Automation. Retrieved September 21, 2003, from http://www.ccla.lib.fl.us/ info/ccla.asp

College Center for Library Automation. (1997). Distance Learning Library Initiative update. Administrative Report, 1. 
College Center for Library Automation. (2003b). First phase of 'Ask a Librarian' service activated. Data_LINCC, 13(4), 1, 6.

College Center for Library Automation. (2002). Grants. Administrative Report, 1.

College Center for Library Automation. (2003c). New “Ask a Librarian” reference service taking shape. Data_LINCC, 13(1), 3.

College Center for Library Automation. (2001). New programs and services. Administrative Report, 1.

Collins, B. C., Schuster, J. W., \& Ludlow, B. L. (2002). Planning and delivery of online coursework in special education. Teacher Education and Special Education, 25, 171-186.

Colvin, G. (2001). Remote, accessible, and on call: Reference librarians go live. Florida Libraries, 44(1), 10-12.

Cooper, J. L. (2000). A model for library support of distance education in the USA. Interlending \& Document Supply, 28, 123-131.

Corrigan, A., Diamond, T., \& Hill, J. B. (2002). Electronic reference service trends among Louisiana academic libraries. Louisiana Libraries, 65(1), 3-12.

Cother, C., \& Parnell, S. (2002, April). Quality assurance and models of service in an environment of change. In P. B. Mahoney (Ed.), The Tenth Off-Campus Library Services Conference proceedings, Cincinnati, OH (pp. 151-163). Mount Pleasant, MI: Central Michigan University.

Cothrel, J., \& Williams, R. L. (1999). On-line communities: Helping them form and grow. Journal of Knowledge Management, 3, 54-60.

Coyner, S. C., \& McCann, P. L. (2004). Advantages and challenges of teaching in an electronic environment. International Journal of Instructional Media, 31, 223-228. Retrieved October 29, 2005, from OmniFile Full Text Mega database.

Cram, J. (1993, July). Operating in the littoral zone: Performance management as a tool of creation. In Infobridges: Linking Australia and Asia: Proceedings of the Second National Reference and Information Service Section Conference, Darwin, Australia.

Cronin, B. (2000, October 15). Customer satisfaction. Library Journal, 125(17), 44.

Crouch, M. L., \& Montecino, V. (1997). Cyberstress: Asynchronous anxiety or worried in cyberspace. In WebNet 98 World Conference of the WWW, Internet, and Intranet proceedings, Orlando, FL. (ERIC Document Reproduction Service No. ED427691) 
Crowley, T. (1985). Half-right reference: Is it true? RQ, 25, 59-68.

Crowley, T., \& Childers, T. (1971). Information service in public libraries: Two studies. Metuchen, NJ: Scarecrow Press.

Cullen, R. (2002). Evolution of information services. Retrieved September 30, 2003, from http://www.facetpublishing.co.uk/inforservs.pdf

Czopek, V. (1998). Using mystery shoppers to evaluate customer service in the public library. Public Libraries, 37, 370-371. Retrieved March 22, 2005, from OmniFile Full Text Mega database.

D'Angelo, B. J., \& Maid, B. M. (1998, December). Service from a distance: The use of information technologies in support of off-campus students. Paper presented at the CAUSE98 Conference, Seattle, WA. Retrieved October 7, 2003, from http://www.educause.edu/ir/library/html/cnc9847/cnc9847.html

Daniel, L. L. (2003). Effects of email on an academic library's reference service. Chapel Hill, NC: University of North Carolina.

Datta, J. (1987). Improving written electronic communications. Administrative Management, 48(8), 21-23.

De Groote, S. L. (2005). Questions asked at the virtual and physical health sciences reference desk: How do they compare and what do they tell us? Medical Reference Services Quarterly, 24(2), 11-23.

Dee, C. R. (2005). Digital reference service: Trends in academic health science libraries. Medical Reference Services Quarterly, 24(1), 19-27.

Deming, L. H. (2000, July/August). Distance learning: One student's perspective. Intercom, 47(7), 18-21.

Dew, S. H. (2000, April). Knowing your users and what they want: Surveying offcampus students about library services. In P. S. Thomas (Ed.), The Ninth OffCampus Library Services Conference proceedings, Portland, OR (pp. 119-132). Mount Pleasant, MI: Central Michigan University.

Dewald, N., Scholz-Crane, A., Booth, A., \& Levine, C. (2000). Information literacy at a distance: Instructional design issues. Journal of Academic Librarianship, 26(1), 33-44.

Dewdney, P. (1986). The effects of training reference librarians in reference skills: A field experiment. Unpublished doctoral dissertation, University of Western Ontario, London, Canada. 
Dewdney, P., Marshall, J. G., \& Tiamiyu, M. (1991). Comparison of legal and health information services in public libraries. $R Q, 31,185-196$.

Dewdney, P., \& Ross, C. S. (1994). Flying a light aircraft: Reference service evaluation from a user's viewpoint. $R Q, 34,217-230$.

Digital Reference Education Initiative. (2004). DREI rubrics for digital reference service providers. Retrieved October 9, 2005, from http://drei.syr.edu/pdf/ DREICompetenciesDraft092004.pdf

Dilevko, J. (2001). An ideological analysis of digital reference service models. Library Trends, 50, 218-244.

Dilevko, J. (2000). Unobtrusive evaluation of reference service and individual responsibility: The Canadian experience. Westport, CT: Ablex. .

Dilevko, J., \& Dolan, E. (1999). Government documents reference service in Canada: Implications for electronic access. Retrieved September 30, 2003, from http://dsp-psd.communication.gc.ca/Rapports/Dilevko_Dolan/dilevko-e.html

Dinwiddie, M., \& Lillard, L. L. (2002, April). At the crossroads: Library and classroom. In P. B. Mahoney (Ed.), The Tenth Off-Campus Library Services Conference proceedings, Cincinnati, $O H$ (pp. 199-211). Mount Pleasant, MI: Central Michigan University.

Dirr, P. J. (1999). Putting principles into practice: promoting effective support services for students in distance learning programs: A report on the findings of a survey. Retrieved September 26, 2003, from http://www.wcet.info/projects/studentservices/ Survey\%20Report.pdf

Donnelly, D. (1995). The digital log. Telematics and Informatics, 12, 131-138.

Druckman, D., Rozelle, R. M., \& Baxter, J. C. (1982). Nonverbal communication: Survey, theory, and research. Beverly Hills, CA: Sage.

Duff, W. M., \& Johnson, C. A. (2001). A virtual expression of need: An analysis of email reference questions. American Archivist, 64, 43-60.

Dunn, S. (2001, January). Fuel for the future. USA Today Magazine, 129(2668), 28-29. Retrieved October 3, 2001, from Academic Search Elite database.

Durrance, J. C. (1989, April 15). Reference success: Does the 55 percent rule tell the whole story? Library Journal, 114(7), 31-36.

Durrance, J. C., \& Fisher, K. E. (2003). Determining how libraries and librarians help. Library Trends, 51, 305-334. 
Dyson, L. J. (1999, April). Improving quality of documents reference service. In Proceedings of the $8^{\text {th }}$ Annual Federal Depository Library Conference, Baltimore, $M D$. Retrieved September 15, 2003, from http://www.access.gpo.gov/su_docs/fdlp/ pubs/proceedings/99pro34.html

Dyson, L. S. (1992). Improving reference services: A Maryland training program brings positive results. Public Libraries, 31, 284-289.

Eastmond, D. V. (1995). Alone but together: Adult distance study through computer conferencing. Cresskill, NJ: Hampton Press.

Edge, S. M., \& Edge, D. (2000, July). Integration of information resources into distance learning programs. Education at a Distance, 14(7). Retrieved September 22, 2001, from http://www.usdla.org/html/journal/JUL00_Issue/PDF/02integration.pdf

Elzy, C., Nourie, A., Lancaster, F. W., \& Joseph, K. M. (1991). Evaluating reference services in a large academic library. College \& Research Libraries, 52, 454-465.

Ewing, K., \& Hauptman, R. (1995). Is traditional reference service obsolete? Journal of Academic Librarianship, 21, 3-6.

Facets of quality for digital reference services, version 5. (2003). Retrieved October 7 , 2003, from http://www.vrd.org/facets-06-03.shtml

Fagan, J. C., \& Calloway, M. (2001). Creating an instant messaging reference system. Information Technology and Libraries, 20, 202-212.

Fallows, D. (2005). Search engine users. Washington: Pew Internet \& American Life Project.

Farmer, L. S. (2005). Virtual reference service for K-12 students. Knowledge Quest, 33(3), 22-24. Retrieved October 8, 2005, from Academic Search Premier database.

Feili-Tu, C. S. (2004). Virtual reference service and school library media specialists. School Library Media Activities Monthly, 20(7), 49-51.

Ferguson, C. D., \& Bunge, C. A. (1997). The shape of services to come: Values-based reference service for the largely digital library. College \& Research Libraries, 58, $252-265$.

Fine, S. (1995). Reference and resources: The human side. Journal of Academic Librarianship, 21, 17-20.

Fishman, D. L. (1998). Managing the virtual reference desk: How to plan an effective reference e-mail system. Medical Reference Services Quarterly, 17(1), 1-10. 
Fister, B. (2002, June 14). Fear of reference. Chronicle of Higher Education, 48(40). Retrieved January 19, 2004, from http://chronicle.com

Florida Department of Education. (2002a). The new Department of Education. Retrieved March 21, 2005, from http://www.fldoe.org/K20/reorg/faqs_7_18_02.asp \#BULLETIN

Florida Department of Education, Division of Accountability, Research, and Measurement. (2005). Report for the Florida Community College System: The fact book. Tallahassee, FL: Author.

Florida Department of Education, Division of Accountability, Research, and Measurement. (2004a). Report for the Florida Community College System: The fact book. Tallahassee, FL: Author.

Florida Department of Education, Division of Colleges and Universities, Office of Academic and Student Affairs. (2004b). An overview of distance and technologymediated instruction in the State University System of Florida. Retrieved March 21, 2005, from http://www.fldcu.org/borpubs/DistanceLearning/DL_Overview200203.pdf

Florida Department of Education, Higher Education Funding Advisory Council. (2002b). Distance learning in Florida. Retrieved March 21, 2005, from http://www.fldoe.org/ HigherEdFundAdvCounc/April_19_02/DistLearning.pdf

Floyd, D. L, \& Casey-Powell, D. (2004). New roles for student support services in distance learning. New Directions for Community Colleges, (128), 55-64.

Foley, M. (2002). Instant messaging reference in an academic library: A case study. College \& Research Libraries, 63, 36-45.

Foster, L., Evans, R. D., \& Lott, C. J. (1997, March). A Degree of Distinction: A Collaborative model for degree delivery via distance education. Paper presented at the annual conference of the American Association of Colleges of Teacher Education, Phoenix, AZ. (ERIC Reproduction Service No. ED408238)

Francouer, S., \& Ellis, L. (2001, November). Information competency standards in chat reference services. Paper presented at the Annual Virtual Reference Desk Conference, Orlando, FL. Retrieved October 17, 2004, from http://www.vrd.org/conferences/VRD2001/proceedings/francoeur.shtml

Frank, I. (1998). E-mail reference service at the University of South Florida: A well-kept secret. Art Documentation, 17(1), 8-9, 44-45. 
Frederiksen, L. (2002, April). Grading ourselves: Using the ACRL Guidelines for Distance Learning Library Services to develop assessment strategies. In P. B. Mahoney (Ed.), The Tenth Off-Campus Library Services Conference proceedings, Cincinnati, $O H$ (pp. 259-264). Mount Pleasant, MI: Central Michigan University.

Frederiksen, L., Cummings, J., \& Ursin, L. (2004). User perceptions and virtual reference services. In R. D. Lankes, J. Janes, L. C. Smith, \& C. M. Finneran (Eds.), Virtual reference experience: Integrating theory into practice (pp. 43-57). New York: NealSchuman.

Fricke, M., \& Fallis, D. (2004). Indicators of accuracy for answers to ready reference questions on the Internet. Journal of the American Society for Information Science and Technology, 55, 238-245.

Fritch, J. W., \& Mandernack, S. B. (2001). The emerging reference paradigm: A vision of reference services in a complex information environment. Library Trends, 50, 286305 .

Fullerton, V. (2002). IFLA digital reference standards project. Retrieved January 11, 2004, from http://www.ifla.org/VII/s36/pubs/drsp.htm

Furst-Bowe, J., \& Dittman, W. (2001). Identifying the needs of adult women in distance learning programs. International Journal of Instructional Media, 28, 405-413.

Gaide, S. (2004a, October 15). Best practices for helping students complete online degree programs. Distance Education Report, 8(20), 8. Retrieved March 20, 2005, from Academic Search Premier database.

Gaide, S. (2004b, April 15). Integrated library services boost online recruitment and retention. Distance Education Report, 8(8), 1-2, 6. Retrieved March 17, 2005, from Academic Search Premier database.

Galusha, J. M. (1998). Barriers to learning in distance education. Hattiesburg, MS: University of Southern Mississippi. (ERIC Document Reproduction Service No. ED416377)

Garamone, J. (2001). Taking the rage out of aggressive driving. American Forces Information Services News. Retrieved March 13, 2004, from http://www.dod.mil/ news/Apr2001/n04092001_200104091.html

Garnsey, B. A., \& Powell, R. R. (2000). Electronic mail reference services in the public library. Reference \& User Services Quarterly, 39, 245-254.

Gay, L. R., \& Airasian, P. (2000). Educational research: Competencies for analysis and application (6th ed.). Upper Saddle River, NJ: Prentice-Hall. 
Gebhard, P. (1997). The reference realist in library academia. Jefferson, NC: McFarland.

George, L. (2002, July/August). E-communities in distance learning. Library Mosaics, 13(4), 14-16.

Gers, R., \& Bolin, N. (1999). More than meets the eye: Management support for reference service and training. Journal of Library Administration, 29(1), 1-15.

Gers, R., \& Seward, L. J. (1985, November 1). Improving reference performance: Results of a statewide study. Library Journal, 110(18), 32-35.

Giannini, T. (1999, May). Modeling the reference process online. In Proceedings of the $20^{\text {th }}$ National Online Meeting, New York, NY (pp. 133-143). Medford, NJ: Information Today.

Goetsch, L. (1995). Reference service is more than a desk. Journal of Academic Librarianship, 21, 15-16.

Goetsch, L., Sowers, L., \& Todd, C. (1999). Electronic reference service. Retrieved January 18, 2004, from http://www.arl.org/spec/251sum.html

Gorry, G. A. (1988). Virtual notebook for biomedical work groups. Bulletin of the Medical Library Association, 76, 257-260.

Gothberg, H. M. (1973). Communication patterns in library reference and information service. $R Q, 13,7-14$.

Green, D. D., \& Peach, J. K. (2003). Assessment of reference instruction as a teaching and learning activity. College \& Research Libraries, 64, 256-258.

Green, S. S. (1876, October). Personal relations between librarians and readers. Library Journal, 1(2/3), 74-81.

Grogan, D. (1992). Practical reference work ( $2^{\text {nd }}$ ed.). London: Library Association Pub.

Gross, M., McClure, C. R., Hodges, R., Graham, A., \& Lankes, R. D. (2001). Assessing quality in digital reference services: Phase II: Site visit summary report. Retrieved September 12, 2003, from http://quartz.syr.edu/quality/default.htm

Gross, M., McClure, C. R., \& Lankes, R. D. (2001). Assessing quality in digital reference services: Overview of key literature on digital reference. Retrieved September 12, 2003, from http://quartz.syr.edu/quality/VRDphaseIILitReviw.pdf

Guerrier, E. (1936, July). The measurement of reference service. Library Journal, 61, 529-531. 
Gupta, K. K. (2001). Librarians' changing role in distance education: Need for training. Journal of Library Administration, 32, 225-231.

Hahn, K. (1997). An investigation of an e-mail-based help service. CLIS Technical Report, (97-03). College Park, MD: College of Library and Information Services, University of Maryland. Retrieved October 7, 2003, from http://www.clis.umd.edu/ research/reports/tr97/03/9703.html

Haines, A., \& Grodzinski, A. (1999). Web forms: Improving, expanding, and promoting remote reference services. College \& Research Libraries News, 60, 271-272.

Hamilton, M. (2000). Distance learning: A new spin on traditional library services. Mississippi Libraries, 64, 67-69. Retrieved September 15, 2003, from http://www.misslib.org/publications/ml/fall00/fa-00.pdf

Hansen, L. (2004, November). Putting it in writing: Potential use and abuse of virtual reference services. In Proceedings of the Virtual Reference Desk Conference 2004, Cincinnati, OH. Retrieved October 8, 2005, from http://www.vrd2004.org/ proceedings/ presentation.cfm?PID $=302$

Hara, N., \& Kling, R. (1999). Students' frustrations with a web-based distance education course: A taboo topic in the discourse. Retrieved December 3, 2003, from http://www.slis.indiana.edu/CSI/WP/wp00_01B.html

Harrington, J., \& Spindle, D. O. (1993, October). Cooperative planning for service and instruction. In Jacob, C. (Ed.), The Sixth Off-Campus Library Services Conference proceedings, Kansas City, MO (pp. 83-96). Mount Pleasant, MI: Central Michigan University.

Harris, L. E. (2004). Software is not enough. In R. D. Lankes, J. Janes, L. C. Smith, \& C. M. Finneran (Eds.), Virtual reference experience: Integrating theory into practice (pp. 109-120). New York: Neal-Schuman.

Hastings, K., \& Tennant, R. (1996, November). How to build a digital librarian. D-Lib Magazine. Retrieved March 17, 2005, from http://www.dlib.org/dlib/november96/ ucb/11hastings.html

He, P. W., \& Knee, M. (1995). The challenge of electronic services librarianship. Reference Services Review, 23(4), 7-12.

Head, M. C., \& Marcella, R. (1993). A testing question: The quality of reference services in Scottish public libraries. Reference Review, 42(6), 7-13.

Head, M. C., Marcella, R., \& Smith, J. M. (1995). Are you being served? The quality of business information provision in Scottish public libraries. Library Review, 44(2), 2837. 
Hébert, F. (1994). Service quality: An unobtrusive investigation of interlibrary loan in large public libraries in Canada. Library and Information Science Research, 16, 3-21.

Henson, B., \& Tomajko, K. G. (2000). Electronic reference services: Opportunities and challenges. Journal of Educational Media \& Library Sciences, 38, 113-121.

Hernon, P. (1996). Service quality in libraries and treating users as customers and nonusers as lost or never-gained customers. Journal of Academic Librarianship, 22(3), 171-172.

Hernon, P. (1987). Utility measures, not performance measures for library reference service? $R Q$ 27, 449-459.

Hernon, P., \& Altman, E. (1996). Service Quality in Academic Libraries. Norwood, NJ: Ablex.

Hernon, P., \& McClure, C. R. (1983). Improving the quality of reference service for government publications. Chicago: American Library Association.

Hernon, P., \& McClure, C. R. (1987a). Unobtrusive testing and library reference services. Norwood, NJ: Ablex.

Hernon, P., \& McClure, C. R. (1986, April 15). Unobtrusive reference testing: The 55 percent rule. Library Journal, 111(7), 37-41.

Hernon, P., \& McClure, C. R. (1987b). Where do we go from here? A final response. Journal of Academic Librarianship, 13(5), 282-284.

Hernon, P., \& Whitman, J. R. (2000). Delivering satisfaction and service quality: A customer-based approach for libraries. Chicago: American Library Association.

Hill, J. B., Madarash-Hill, C., \& Bich, N. P. (2003). Digital reference evaluation: Assessing the past to plan for the future. Electronic Journal of Academic and Special Librarianship, 4(2-3), 1-13. Retrieved October 20, 2004, from http://southernlibrarianship.icaap.org/content/v04n03/Hill_j01.htm

Hill, J. B., \& Stahr, B. (2003). Instructional opportunities in virtual reference. In Teaching in higher education forum e-proceedings. Baton Rouge, LA: Louisiana State University. Retrieved September 29, 2003, from http://www.celt.lsu.edu/CFD/EProceedings/Instructional\%20Opportunities\%20in\%20Virtual\%20Reference

Hirko, B., Foley, N., \& Pitney, B. (2004, November). Practical evaluation: Not an oxymoron! In Proceedings of the Virtual Reference Desk Conference 2004, Cincinnati, OH. Retrieved October 8, 2005, from http://www.vrd2004.org/ proceedings/hirko_files/frame.htm 
Hodel, M. A. (2004, April). Future of reference? Paper presented at Realities of Digital Reference: Challenges, Tools, and Opportunities Teleconference. Retrieved October 31, 2004, from http://www.cod.edu/teleconf/VIRTUAL/slides2.htm\#Hodel

Hodges, R. A. (2002). Assessing digital reference. Libri, 52, 157-168.

Horn, J., \& Kjaer, K. (2000). Evaluating the "Ask a Question" service at the University of California, Irvine. In R. D. Lankes, J. W. Collins, \& A. S. Kasowitz (Eds.), Digital reference service in the new millennium (pp. 135-152). New York: Neal-Schuman.

Horner, J., \& Michaud-Oystryk, N. (1995). The efficiency and success rates of print ready reference vs online ready reference searches in Canadian university libraries. Journal of Academic Librarianship, 21(2), 97-102.

Howard, E. H., \& Jankowski, T. A. (1986). Reference services via electronic mail. Bulletin of the Medical Library Association, 74, 41-44.

Hubbertz, A. (2005). The design and interpretation of unobtrusive evaluations. Reference \& User Services Quarterly, 44, 327-335.

Hufford, J. R. (2001). Planning for distance learning: Support services and the library's role. Journal of Library Administration, 32, 259-266.

Hufford, J. R. (2000). The university library's role in planning a successful distance learning program. Reference Librarian, 33(69/70), 193-203.

Hults, P. (1992). Reference evaluation: An overview. Reference Librarian, (38), 141-150.

Hutchinson, D., \& Pye, M. (2004, November). We've counted it - Now what?: Establishing performance targets for a virtual reference service. Paper presented at the Virtual Reference Desk Conference, Cincinnati, OH. Retrieved November 12, 2005, from http://www.vrd2004.org/proceedings/presentation.cfm?PID=383

Institute for Higher Education Policy. (2000, April). Quality on the line: Benchmarks for success in internet-based education. Retrieved December 6, 2003, from http://www.ihep.com/Pubs/PDF/Quality.pdf

Internet Public Library. (2005). IPL 10th - Timeline. Retrieved March 25, 2005, from http://www.ipl.org/div/anniversary/timeline.html

Jackson, M., \& Parker, S. (1998). The role of the library and information services in supporting students in resource-based learning: Some findings of the IMPEL 2 project. Journal of Further and Higher Education 22, 173-181. 
Jackson, M. G. (2002). A primary knowledge revolution: New demands, new responsibilities for reference librarians and services in the digital age. Library Quarterly, 72, 123-128.

Jahoda, G., \& Braunagel, J. S. (1980). The librarian and reference queries: A systematic approach. New York: Academic Press.

Janes, J. (2002a). Digital reference: Reference librarians' experiences and attitudes. Journal of the American Society for Information Science and Technology, 53, 549566.

Janes, J. (2000). Digital reference: Services, attitudes, and evaluation. Internet Research, $10,256-258$.

Janes, J. (2001). Digital reference services in public and academic libraries. In C. McClure \& J. C. Berot (Eds.), Evaluating networked information services: Techniques, policy and issues (pp. 175-195). Medford, NJ: Information Today.

Janes, J. (2004a). Integration of digital reference. In R. D. Lankes, J. Janes, L. C. Smith, \& C. M. Finneran (Eds.), The virtual reference experience: Integrating theory into practice (pp. 1-7). New York: Neal-Schuman.

Janes, J. (2003). Introduction to reference work in the digital age. New York: NealSchuman.

Janes, J. (2002b, October 15). Live reference: Too much, too fast? Library Journal netConnect, 12-14. Retrieved September 28, 2003, from http://libraryjournal.reviewsnews.com/ index.asp?layout=articlePrint\&article $\mathrm{ID}=\mathrm{CA} 251681$

Janes, J. (2002c, November). Question negotiation in an electronic age. In Electronic proceedings - 2002 VRD Conference, Chicago, IL. Retrieved March 17, 2005, from http://www.vrd.org/conferences/VRD2002/proceedings/janes.shtml

Janes, J. (2004b, April). Where we are? Paper presented at Realities of Digital Reference: Challenges, Tools, and Opportunities Teleconference. Retrieved October 31, 2004, from http://www.cod.edu/teleconf/VIRTUAL/slides2.htm\#Janes

Janes, J., Carter, D., \& Memmott, P. (1999). Digital reference services in academic libraries. Reference \& User Services Quarterly, 39, 145-150.

Janes, J., \& Silverstein, J. (2003, February). Question negotiation and the technological environment. D-Lib Magazine, 9(2). Retrieved October 31, 2003, from http://www.dlib.org/dlib/february03/janes/02janes.html 
Jensen, A., \& Sih, J. (1995). Using e-mail and the Internet to teach users at their desktops. Online, 19(5), 82-86.

Jensen, B. (2004). The case for non-intrusive research: A virtual reference librarian's perspective. The Reference Librarian, (85), 139-149.

Jesudason, M. (2000). Outreach to student-athletes through e-mail reference service. Reference Services Review, 28, 262-267.

Jirjees, J. M. (1981). Accuracy of selected northeastern college library reference/information telephone services in responding to factual inquiries (Doctoral dissertation, Rutgers University, 1981). Dissertation Abstracts International, 42, 6.

Johnson, C. A., \& McCarty, L. (2001). Distance education and digital reference: The yellow brick road? ALKI, 17(1), 6-9.

Johnson, W. T. (1999). Library support for distance learning. Community \& Junior College Libraries, 8(2), 51-57.

Johnston, P., \& Grusin, A. (1995). Personal service in an impersonal world: Throwing life preservers to those drowning in an ocean of information. Georgia Librarian, 32, 45-49.

Jonassen, D. H., Peck, K. L., \& Wilson, B. G. (1998). Creating technology-supported learning communities. Retrieved December 2, 2003, from http://carbon.cudenver.edu/ $\sim$ bwilson/learncomm.html

Jonassen, D. H., Peck, K. L., \& Wilson, B. G. (1999). Learning with technology: A constructivist perspective. Upper Saddle River, NJ: Prentice Hall.

Jones, M. F. (2003.). Getting started: A guide for new distance learning librarians. Retrieved December 12, 2003, from http://caspian.switchinc.org/ distlearn/ guidelines/getting_started.pdf

Jones, S. (2002, September). The Internet goes to college: How students are living in the future with today's technology. Washington: Pew Internet and American Life Project. Retrieved July 31, 2003, from http://www.pewinternet.org/reports/pdfs/ PIP_College_Report.pdf

Kahn, M. J. (2001). Using qualitative criteria to evaluate reference resources. In D. P. Wallace \& Van Fleet, C. (Eds.), Library evaluation: A casebook and can-do guide (pp. 131-137). Englewood, CO: Libraries Unlimited.

Kanuka, H., Collett, D., \& Caswell, C. (2002). University instructor perceptions of the use of asynchronous text-based discussion in distance courses. American Journal of Distance Education, 16, 151-167. 
Kaske, N., \& Arnold, J. (2002). Unobtrusive evaluation of online real time library reference service. Retrieved September 14, 2003, from http://www.lib.umd.edu/groups/digref/kaskearnoldunobtrusive.html

Kasowitz, A. S., Bennett, B. A., \& Lankes, R. D. (2000). Quality standards for digital reference consortia. Reference \& User Services Quarterly, 39, 355-363.

Katz, W. A. (2002). Introduction to reference work, volume 1: Basic information sources $\left(8^{\text {th }}\right.$ ed.). Boston: McGraw-Hill.

Katz, W. A. (2001). Long live old reference services and new technologies. Library Trends, 50, 263-285.

Kawakami, A. K. (2002, Spring). Delivering digital reference. Library Journal NetConnect, 127(7), 28-29.

Keith, E., \& Kohut, D. (1998). Reference and bibliographic instruction: A survey of philosophy statements in LIBRAS libraries. Illinois Libraries, 80(2), 57-59. Retrieved August 15, 2005, from OmniFile Full Text Mega Edition database.

Kellehear, A. (1993). Unobtrusive researcher: A guide to methods. Sydney: Allen and Unwin.

Kenney, B. (2002, October 1). Live, digital reference. Library Journal, 127(16). Retrieved January 18, 2003, from http://www.libraryjournal.com/index.asp? layout $=$ articlePrint\&articleID $=\mathrm{CA} 245058$

Kern, M. K. (2004). Chat it up! Extending reference services to assist off-campus students. Journal of Library Administration, 41, 217-226.

Kern, M. K., \& Gillie, E. (2004). Virtual reference policies: An examination of current practice. In R. D. Lankes, J. Janes, L. C. Smith, \& C. M. Finneran (Eds.), The virtual reference experience: Integrating theory into practice (pp. 165-184). New York: Neal-Schuman.

Kibbee, J., Ward, D., \& Ma, W. (2002). Virtual service, real data: Results of a pilot study. Reference Services Review, 30, 25-36.

Kiernan, V. (2003, July 21). A survey documents growth in distance education in late 1990s. Chronicle of Higher Education, 1-2. Retrieved July 21, 2003, from http://chronicle.com/daily/2003/07/2003072102t.htm

King, G., \& Berry, G. (1973). Evaluation of the University of Minnesota Libraries Reference Department telephone information study. Pilot study. Minneapolis, MN: University of Minnesota Library School. (ERIC Document Reproduction Service No. ED077517) 
Kochtanek, T. R., \& Hein, K. K. (2000). Creating and nurturing distributed asynchronous learning environments. Online Information Review, 24, 280-293.

Kong, L. M. (1995). Reference service evolved. Journal of Academic Librarianship, 21(1), 13-14.

Kosmin, L. J. (1990, May). Electronic reference desk: Prospects, promises, realities. In Proceedings of the National Online Meeting, New York, NY (pp. 217-224). Medford, NJ: Learned Information.

Krauth, B., \& Carbajal, J. (1999). Guide to developing online student services. Retrieved December 31, 2001, from http:/www.wiche.edu/Telecom/resources/ publications/guide/guide.htm

Kresh, D. N. (2000, June). Offering high quality reference service on the Web. D-Lib Magazine, 6(6). Retrieved October 7, 2003, from http://www.dlib.org/dlib/june00/ kresh/06kresh.html

Kuhlthau, C. (1988). Developing a model of the library research process: Cognitive and affective aspects. $R Q, 28,232-242$.

Kwon, N. (2006). User satisfaction with referrals at a collaborative virtual reference service. Information Research, 11(2), 1-20. Retrieved December 15, 2005, from http://informationr.net/ir/11-2/paper246.html

Ladner, S. J., \& Tillman, H. N. (1993, January). Using the Internet for reference. Online, $17(1), 45-51$.

Lagace, N., \& McClennen, M. (1998, February). Questions and quirks: Managing an Internet-based distributed reference service. Computers in Libraries, 18(2), 24-27. Retrieved October 7, 2003, from http:/www.infotoday.com/cilmag/feb98/story1.htm

Lamolinara, G., \& Grunke, R. (1998, February). Reference service in a digital age. LC Information Bulletin, 57(2). Retrieved October 8, 2003, from http://www.loc.gov/loc/ lcib/9808/ref.html

Lankes, R. D. (1998). Building and maintaining internet information services: K-12 digital reference services. Syracuse, NY: ERIC Clearinghouse on Information \& Technology at Syracuse University. (ERIC Document Reproduction No. ED427778)

Lankes, R. D. (2004). The digital reference research agenda. Journal of the American Society for Information Science and Technology, 55, 301-311. 
Lankes, R. D. (2003). Impact and opportunity of digital reference in primary and secondary education in the digital reference research agenda. In R. D. Lankes, A. Goodrum, \& S. Nicholson (Eds.), Publications in librarianship (pp. 61-83). Chicago: Association of College and Research Libraries.

Lankes, R. D. (2002, June). Setting the standards in digital reference: Defining quality and performance measures. Paper presented at RUSA/MARS pre-conference at the ALA Annual Conference, Atlanta, GA. Retrieved January 17, 2004, from http://quartz.syr.edu/rdlankes/tiki-download_file.php?fileId=55

Lankes, R. D., Collins, J. W., \& Kasowitz, A. (2000). Digital reference service in the new millennium. New York: Neal-Schuman.

Lankes, R. D., Gross, M., \& McClure, C. R. (2003). Cost, statistics, measures, and standards for digital reference services: A preliminary view. Library Trends, 51, 401-413.

Lankes, R. D., Janes, J., Smith, L. C., \& Finneran, C. M. (Eds.). (2004). Virtual reference experience: Integrating theory into practice. New York: NealSchuman.

LaPadula, M. (2003). A comprehensive look at online student support services for distance learners. American Journal of Distance Education, 17, 119-128.

Larson, M. E. (1990). Connecting to the electronic library: A paradigm shift in training reference librarians. Reference Librarian, (30), 97-104.

Laukenmann, M., et. al. (2003). An investigation of the influence of emotional factors on learning in physics instruction. International Journal of Science Education, 25, 489507.

Lawson, M. D., Lillard, L., Antrim, P., \& Morgan, S. (2000). We're in this together: Librarians as co-instructors with classroom faculty for electronic delivery. In W. Winfield (Ed.), Distance Learning 2000: Conference proceedings, Madison, WI (pp. 251-255). Madison, WI: University of Wisconsin, Madison.

Lebowitz, G. (1997). Library services to distant students: An equity issue. Journal of Academic Librarianship, 24, 303.

Lee, I. J. (2004). Do virtual reference librarians dream of digital reference questions?: A qualitative and quantitative analysis of Email and chat reference. Australian Academic Libraries, 35, 95-110.

Lefor, P. J., Benke, M., \& Ting, E. (2003). Information technology and adult learners at Empire State College. New Directions for Student Services, (102), 35-42. 
Lessin, B., McGinnis, R., \& Bean, R. (2002). Guidelines and standards applicable for library services to distance education business programs. Journal of Business \& Finance Librarianship, 7(2/3), 155-205.

Lester, J., \& Van Fleet, C. (2003). Oklahoma's certification for public librarians program and the Institute in Public Librarianship. Public Libraries, 42, 245-251.

Lewis, D. W. (1995). Traditional reference is dead, Now let's move on to important questions. Journal of Academic Librarianship, 21(1), 10-12.

Lipow, A. G. (2003). The future of reference: Point of need reference service: No longer an afterthought. Reference Services Review, 31(1), 31-35.

Lipow, A. G. (1999, August 1). "In your face” reference service. Library Journal, 124(13), 50-52.

Lock, J. (2002). Laying the groundwork for the development of learning communities within online courses. Quarterly Review of Distance Education, 3(4), 395-408.

Lorenzetti, J. (2002a, April 15). Before they drift away: Two experts pool retention insights. Distance Education Report, 6(8), 1.

Lorenzetti, J. P. (2004, July 15). Recognizing the challenges of distance ed. Distance Education Report, 8(14), 3. Retrieved October 24, 2005, from Academic Search Premier database.

Lorenzetti, J. P. (2002b, November 15). Well begun is half done: Is orientation a key to retention? Distance Education Report, 6(22), 1, 6.

Love-Rodgers, C. (2001). Electronic resources for the arts: Supporting distance learners at the Open University. Art Libraries Journal, 26(3), 4-7.

Ludwig, L. (2002). Essential library support for distance education. Retrieved August 9, 2003, from http://www.mlanet.org/government/positions/disteduc_2.html

Lynch, B. P. (2002, October 7). Digital divide or the digital connection: A U.S. perspective. First Monday, 7(10). Retrieved January 6, 2004, from http://firstmonday.org/issues/issue7_10/lynch/index.html

Ma, W., \& Wright, J. C. (1998). QB online: How an old-fashioned question board went electronic at the University of Illinois. College \& Research Libraries News, 59, $772-774$.

Madaus, J. R., \& Webster, L. (1998, May). Opening the door to distance learning. Computers in Libraries, 18(5), 51-55. 
Mahony, A. P. (1993). Net and reference services: Capture the question. Wilson Library Bulletin, 69(2), 12.

Markgraf, J. S. (2002, April). Collaboration between distance education faculty and the library: One size does not fit all. In P. B. Mahoney (Ed.), The Tenth Off-Campus Library Services Conference proceedings, Cincinnati, OH (pp. 351-360). Mount Pleasant, MI: Central Michigan University.

Marsteller, M. R., \& Mizzy, D. (2003). Exploring the synchronous digital reference interaction for query types, question negotiation, and patron response. Internet Reference Services Quarterly, 8, 149-165.

Martin, L. A., Crowley, T., \& Shaughnessy, T. (1969). Library response to urban change: A study of the Chicago Public Library. Chicago: American Library Association.

Massey-Burzio, V. (2002). Facing the competition: The critical issues of reference service. College \& Research Libraries News, 63, 774-775.

Massey-Burzio, V. (1998). From the other side of the reference desk: An interview study. Journal of Academic Librarianship, 24(3), 208-215.

McClure, C. R. (2004). Challenges and strategies for evaluating networked information services: Introduction. Library Quarterly, 74, 399-402.

McClure, C. R. (1980). Information for academic library decision making: The case for organizational information management. Westport, CT: Greenwood.

McClure, C. R., \& Lankes, R. D. (2001). Assessing quality in digital reference services: A research prospectus. Retrieved December 10, 2003, from http://quartz.syr.edu/quality/Overview.htm

McClure, C. R., Lankes, R. D., Gross, M., \& Choltco-Devlin, B. (2002). Statistics, measures, and quality standards for assessing digital reference library services: Guidelines and procedures. Syracuse, NY: Information Institute of Syracuse. (ERIC Document Reproduction Service No. ED472588)

McClure, C. R., \& Lopata, C. L. (1996). Assessing the academic networked environment: Strategies and options. Washington: Coalition of Networked Information.

McGlamery, S., \& Coffman, S. (2000). Moving reference to the Web. Reference \& User Services Quarterly, 39, 380-386.

McGranahan, J. (2005). Virtual reference. Library Mosaics, 16(2), 16. Retrieved October 8, 2005, from Academic Search Premier database. 
McLean, E. (2000). Student support with particular reference to library support: The case of the University of West Indies Mona Campus Library. Retrieved August 17, 2003, from http://www.col.org/resources/publications/SmallStates00/2_conf_proc_ McLean.pdf

Mellon, C. (1986). Library anxiety: A grounded theory and its development. College \& Research Libraries, 47, 160-165.

Middleton, M., \& Peacock, J. (2000). Library services to external students from Australian universities: The influence of flexible delivery upon traditional service provision. Reference Librarian, 33(69/70), 205-217.

Milam, P. (2005). Your ticket to success. School Library Journal.com, 51(3), 52-54. Retrieved March 25, 2005, from http://www.schoollibraryjournal.com/article/ CA507331.html

Mills, M., Fisher, C., \& Stair, N. (2001). Web-based courses: More than curriculum. Nursing \& Health Care Perspectives, 22, 235-239.

Missingham, R. (2000). Report on the Virtual Reference Desk Conference October 2000, Seattle, Washington. Retrieved January 18, 2004, from http://www.dlib.org/dlib/december00/12missingham.html

Moisey, S. D. (2004). Students with disabilities in distance education. Journal of Distance Education, 19(1), 73-91.

Moore, M. G. (1989). Three types of interaction. American Journal of Distance Education, 3(2), 1-6.

Moran, R. F. (2003). ALA/APA Post-Master's Certification. Library Administration \& Management, 17, 4-5.

Morin, A. C. (2004). Approaching best practices and guidelines for digital reference. In R. D. Lankes, J. Janes, L. C. Smith, \& C. M. Finneran (Eds.), The virtual reference experience: Integrating theory into practice (pp. 185-198). New York: NealSchuman.

Moss, M. M. (1997). Reference services for remote users. Katharine Sharp Review, (5). Retrieved October 7, 2003, from http://www.lis.uiuc.edu/review/5/moss.html

Mudrock, T. (2002). Revising ready reference sites. Reference \& User Services Quarterly, 42, 155-163.

Murfin, M. E. (1995). Evaluation of reference service by user report of success. Reference Librarian, (49/50), 229-241. 
Murfin, M., \& Bunge, C. (1984). Evaluating reference service from the patron point of view: Some interim national survey results. Reference Librarian, 11, 175-182.

Myers, M. J., \& Jirjees, J. M. (1983). The accuracy of telephone reference/information services in academic libraries. Metuchen, NJ: Scarecrow Press.

Nash, P. (2000, February). Presentation summary and evaluation results. Retrieved January 17, 2004, from http://www.vrd.org/conferences/VRD99/summary.shtml

New England Association of Colleges and Schools. (2001). Commission on Institutions of Higher Education standards for accreditation. Retrieved November 1, 2003, from http://www.neasc.org/cihe/stancihe.htm

Nilsen, K. (2004). The library visit study: User experiences at the virtual reference desk. Information Research, 9(2), 1-15. Retrieved July 12, 2004, from http://informationr.net/ir/9-2/paper171.html

Norlin, E. (2000). Reference evaluation: A three-step approach - Surveys, unobtrusive observations, and focus groups. College \& Research Libraries, 61, 546-553.

Novotny, E. (2001). Evaluating electronic reference services: Issues, approaches and criteria. Reference Librarian, 35(74), 103-120.

OCLC. (2002). How academic librarians can influence students' web-based information choices. Retrieved November 15, 2002, from http://www2.oclc.org/ oclc/pdf/printondemand/informationhabits.pdf

OCLC. (2003). The 2003 OCLC environmental scan pattern recognition. Retrieved January 10, 2004, from http://www.oclc.org/membership/escan/introduction/ default.htm

Oder, N. (2003, April 15). Internet PL faces money crunch. Library Journal, 128(7), 17.

Oder, N. (2001, February 1). The shape of e-reference. Library Journal, 126(2), 46-50.

Ojala, M. (2003, March/April). Certifying information professionals. Online, 27(2). Retrieved March 25, 2003, from http://www.infotoday.com/online/mar03/ homepage.shtml

Olsen, J. M. (2001). Online library services: Elements of style. In C. Dalziel \& M. Payne (Eds.), Quality enhancing practices in distance education: Student services (pp. 6266). Washington: Instructional Telecommunications Council.

Oregon University System. (1995). Library support for distance education programs. Retrieved August 9, 2003, from http://www.ous.edu/dist-learn/library.htm 
O’Sullivan, M., \& Scott, T. (2000). Teaching Internet information literacy: A critical evaluation. Multimedia Schools, 7(3), 34-37.

Palloff, R. M., \& Pratt, K. (1999). Building learning communities in cyberspace: Effective strategies for the online classroom. San Francisco: Jossey-Bass Publishers.

Parsad, B., \& Lewis, L. (2003). Remedial education at degree-granting postsecondary institutions in fall 2000. Washington: U.S. Department of Education, Institute of Education Sciences, National Center for Education Statistics. Retrieved January 14, 2004, from http://nces.ed.gov/pubs2004/2004010.pdf

Parus, D. J. (1996). Reference interview: Communication and the patron. Katharine Sharp Review, (2). Retrieved October 31, 2003, from http://alexia.lis.uiuc.edu/ review/winter1996/parus.html

Paskoff, B. M. (1991). Accuracy of telephone reference service in health sciences libraries. Bulletin of the Medical Library Association, 79, 182-188.

Pease, B., \& Power, C. (1994). Reference services for off-campus students and faculty. Reference Librarian, (43), 43-62.

Peters, T. A. (2000). Current opportunities for the effective meta-assessment of online reference services. Library Trends, 49, 334-349.

Philip, B. (1997). An examination of the past, present and future. Retrieved October 7, 2003, from http://hollyhock.slis.ualberta.ca/598/brenda/emailref.htm

Pierce, S. (1984). In pursuit of the possible: Evaluating reference services. In B. Katz \& R. Fraley (Eds.), Evaluation of reference services (pp. 53-61). New York: Haworth Press.

Plotnick, A. (1985). Half-right reference. American Libraries, 16, 227.

Pomerantz, J. (2003). Integrating digital reference service into the digital library environment in the digital reference research agenda. In R. D. Lankes, A. Goodrum, \& S. Nicholson (Eds.), Publications in librarianship (pp. 23-47). Chicago: Association of College \& Research Libraries.

Pomerantz, J., Luo, L., \& McClure, C. R. (in press). Peer review of chat reference transcripts: Approaches and strategies. Library \& Information Science Research, 28(1). Retrieved October 9, 2005, from http://ils.unc.edu/ jpom/pubs/Preprint-LISR2006.pdf

Pomerantz, J., Nicholson, S., Belanger, Y., \& Lankes, R. D. (2004). The current state of digital reference: Validation of a general digital reference model through a survey of digital reference services. Information Processing and Management, 40, 347-363. 
Powell, C. A., \& Bradigan, P. S. (2001). E-mail reference services. Reference \& User Services Quarterly, 41, 170-178.

Quint, B. (2004, June). Rules of ruling the road. Information Today,21(6), 7. Retrieved August 13, 2005, from Academic Search Premier database.

Radford, M., \& Thompson, J. (2004, June). Hmmm, just a moment while I keep Looking: Interpersonal communication in chat. Panel presentation at the annual conference of the American Library Association, Orlando, FL.

Ratcliff, J. L. (1996). Realizing the potential: Improving postsecondary teaching, learning, and assessment. University Park, PA: National Center on Postsecondary Teaching, Learning, and Assessment.

Restauri, S. L. (2004). Creating an effective online distance education program using targeted support factors. TechTrends, 48(6), 32-39. Retrieved March 20, 2005, from Academic Search Premier database.

Rettig, J. (1996). The conversion of the Twain or Titanic collision? Bibliographic instruction and reference in the 1990's sea of change. In L. Shirato \& R. Fowler (Eds.), Change in reference and bibliographic instruction: How much help and how? (pp. 3-9). Ann Arbor, MI: Pierian Press.

Rice, R. E. (1993). Media appropriateness: Using social presence theory to compare traditional and new organizational media. Human Communication Research, 19, 451-484.

Richardson, J. V., Jr. (1999a, May). Modeling the reference process: A systems approach. College \& Research Libraries, 60, 211-222.

Richardson, J. V., Jr. (2002, April 15). Reference is better than we thought. Library Journal, 127(7), 41-42.

Richardson, J. V., Jr. (1999b). Understanding the reference transaction: A systems analysis perspective. College \& Research Libraries, 60, 211-222.

Riechel, R. (1986). The telephone patron and the reference interview: The public library experience. Reference Librarian, (16), 81-88.

Roberts, H. V., \& Sergesketter, B. E. (1992). Quality is personal: A foundation for total quality management. New York: Free Press.

Roberts, S., \& Davey, J. (2002). VLEs and information services: Redefining distance learning and the role of information services within the virtual learning environment. In P. Brophy, S. Fisher, and Z. Clark (Eds.), Libraries without walls 4: The delivery of library services to distant users (pp. 73-84). London: Facet Publishing. 
Rodman, R. L. (2003). Cost analysis and student survey results of library support for distance education. Journal of the Medical Library Association, 91, 72-78.

Rodman, R. L. (2001). The S.A.G.E. Project: A model for library support of distance education. Internet Reference Services Quarterly, 6(2), 35-45.

Ronan, J. (2003). The reference interview online. Reference \& User Services Quarterly, $43,43-47$.

Ross, C. S., \& Dewdney, P. (1998). Negative closure: Strategies and counterstrategies in the reference transaction. Reference \& User Services Quarterly, 38, 151-163.

Ross, C. S., \& Nilsen, K. (2000). Has the Internet changed anything in reference? The library visit study, phase 2. Reference \& User Services Quarterly, 40, 147-155.

Ross, C. S., Nilsen, K., \& Dewdney, P. (2002). Conducting the reference interview: A how to do it manual for librarians. New York: Neal Schuman.

Roy, L. (1995). Reference accuracy. Reference Librarian, (49/50), 217-227.

Roysdon, C. M., \& Elliott, L. L. (1988). Electronic integration of library services through a campuswide network. $R Q, 28,82-93$.

Rumble, G. (2000). Student support in distance education in the $21^{\text {st }}$ century: Learning from service management. Distance Education, 21(2), 216-235.

Ryer, M. A., \& Nebeker, B. (1999). Implementing an "Ask a Librarian" electronic reference service. Community \& Junior College Libraries, 9(1), 21-34.

Sacks, P. (1996). Generation X goes to college: An eye-opening account of teaching in postmodern America. Chicago: Open Court.

Salem, Jr., J. A., Balraj, L. E., \& Lilly, E. B. (2004). Real-time training for virtual reference. In R. D. Lankes, J. Janes, L. C. Smith, \& C. M. Finneran (Eds.), The virtual reference experience: Integrating theory into practice (pp. 121-138). New York: Neal-Schuman.

Sampson, J. P. (1999). Integrating Internet-based distance guidance with services provided in career centers. Career Development Quarterly, 47, 243-254.

Santovec, M. L. (2002, December 15). Identifying key success factors in online programs. Distance Education Report, 6(24), 8.

Sarkodie-Mensah, K. (1997). Human side of reference in an era of technology. Reference Librarian, (59), 131-138. 
Saxton, M., Kaiser, R., \& Reichert, J. (2004, November). Show me yours and I'll show you mine! Implementing peer review. Proceedings of the Virtual Reference Desk Conference 2004, Cincinnati, OH. Retrieved October 8, 2005, from http://www.vrd2004.org/proceedings/presentation.cfm?PID=401

Saxton, M. L., \& Richardson, J. V., Jr. (2002). Understanding reference transactions: Transforming an art into a science. New York: Academic Press.

Scagnoli, N. I. (2001). Student orientations for online programs. Journal of Research on Technology in Education, 34, 19-27.

Schilling-Eccles, K., \& Harzbecker, J. J. (1998). The use of electronic mail at the reference desk: Impact of a computer-mediated communication technology on librarian-client interactions. Medical Reference Services Quarterly, 17(4), 17-27.

Schloman, B. (1993). Managing reference services in an electronic environment. Reference Librarian, (39), 99-109.

Schneider, K. G. (2000). My patron wrote me a letter: The joy of e-mail reference. American Libraries, 31(1), 96.

Schroeder, E. E., \& Zarinnia, E. A. (1999, March). Argumentation online: The use of computer conferencing. In J. D. Price, J. Willis, D. A. Willis, M. Jost, \& S. BogerMehall (Eds.), Proceedings of Society for Information Technology \& Teacher Education, San Antonio, TX (pp. 1887-1893). Charlottesville, VA: Association for the Advancement of Computing in Education.

Schwartz, D. G., \& Eakin, D. (1986). Reference service standards, performance criteria, and evaluation. Journal of Academic Librarianship, 12(1), 4-8.

Schweitzer, C. (2003, May). Customize, don't dehumanize. Association Management, 55, 47-52.

Sears, J. (2001). Chat reference service: An analysis of one semester's data. Issues in Science and Technology Librarianship. Retrieved January 3, 2004, from http://www.istl.org/istl/01-fall/article2.html

Seay, T., Seaman, S., \& Cohen, D. (1996). Measuring and improving the quality of public services: A hybrid approach. Library Trends, 44, 464-490.

Shamel, C. L. (2001). Centralized library and learning resources: A remote access demonstration project. Community \& Junior College Libraries, 10(4), 13-28.

Sheets, M. F. (1992). Characteristics of adult education students and factors which determine course completion: A review. New Horizons in Adult Education, 6(2). Retrieved January 12, 2002, from http://www.nova.edu/ aed/horizons/vol6n1 
Sherritt, C. (1996). Fundamental probe with distance programs in higher education. (ERIC Document Reproduction Service No. ED389906)

Simmonds, P. L., \& Andaleeb, S. S. (2001). Usage of academic libraries: The role of service quality, resources, and user characteristics. Library Trends, 49, 626-634.

Singh, P., \& Pan, W. (2004). Online education. College Student Journal, 38, 302-308. Retrieved October 29, 2005, from OmniFile Full Text Mega database.

Sjolander, E., \& Sjolander, R. (1995). A strategic analysis of the delivery of service in two library reference departments. College \& Research Libraries, 56, 60-70.

Slade, A. L. (2001). Quality assurance in library support of distance learning: International perspectives for library administrators. Advances in Library Administration and Organization, 18, 225-244.

Sloan, B. (2002, November). Asking questions in the digital library: Can users define a VR service? In Proceedings of the Virtual Reference Desk Conference 2002, Chicago, IL. Retrieved January 17, 2004, from http://www.vrd.org/conferences/VRD2002/ proceedings/sloan.shtml

Sloan, B. (1998a). Electronic reference services: Some suggested guidelines. Reference \& User Services Quarterly, 38, 77-81.

Sloan, B. (2003). My web librarian: A report on patterns of system use and user satisfaction. Retrieved July 12, 2004, from http://wwwlis.uuc.edu/ bsloan/mwlreport.htm

Sloan, B. (1998b). Service perspective for digital library: Remote reference services. Library Trends, 47, 117-143.

Smith, L. C. (2003). Education for digital reference services in the digital reference research agenda. In R. D. Lankes, A. Goodrum, \& S. Nicholson (Eds.), Publications in librarianship (pp. 149-176). Chicago: Association of College \& Research Libraries.

Smith, R., Race, S. F., \& Ault, M. (2001). Virtual desk: Real reference. Journal of Library Administration, 32, 371-382.

Southern Regional Education Board. (2002a). Principles of good practice: The foundation for quality of the Electronic Campus of the Southern Regional Education Board, 2002-2003. Retrieved January 10, 2004, from http://www.electroniccampus.org/student/srecinfo/publications/principles.asp 
Southern Regional Education Board, Distance Learning Policy Laboratory. (2002b). Anytime, anyplace services for the $21^{\text {st }}$ century student. Retrieved March 21, 2005, from http://www.ecinitiatives.org/policylab/Reports/Student\%20services\%20Final $\% 205.31 .02 \% 20$ USE.pdf

Sproull, L. S., \& Kiesler, S. (1986). Reducing social context cues: Electronic mail in organizational communication. Management Science, 32, 1492-1512.

Stacy-Bates, K. (2003). E-mail reference responses from academic ARL libraries. Reference \& User Services Quarterly, 43, 59-70.

Stahl, J. (1998). "Have a question? Click here": Electronic reference at the National Museum of American Art. Art Documentation, 17(1), 10-12.

Staley, L. (1998). E-mail reference: Experiences at City University. PNLA Quarterly, 62(4), 20-21.

Stalker, J. C., \& Murfin, M. E. (1996). Quality reference service: A preliminary case study. Journal of Academic Librarianship, 22(6), 423-429.

Stanley, D., \& Lyandres, N. (2001). Reference assistance to remote users. Reference Librarian, (73), 243-252.

Stemper, J. A., \& Butler, J. T. (2001). Developing a model to provide digital reference services. Reference Services Review, 29, 172-188.

Stephens, K. (1996). Role of the library in distance learning: A review of UK, North American and Australian literature. New Review of Academic Librarianship, 2, 205234.

Still, J., \& Campbell, F. (1993). Librarian in a box: The use of electronic mail for reference. Reference Services Review, 21(1), 15-18.

Stillwell, M., \& Moore, M. (2002). Reference transformed: State Library initiative proves its worth. ALKI, 18(3), 24-25.

Stormont, S. (2001). Going where the users are: Live digital reference. Information Technology and Libraries, 20, 129-134.

Straw, J. E. (2004). Expecting the stars but getting the moon. In R. D. Lankes, J. Janes, L. C. Smith, \& C. M. Finneran (Eds.), The virtual reference experience: Integrating theory into practice (pp. 87-106). New York: Neal-Schuman.

Straw, J. E. (2001). From magicians to teachers: The development of electronic reference in libraries: 1930-2000. Reference Librarian, 35(74), 1-12. 
Straw, J. E. (2000). A virtual understanding: The reference interview and question negotiation in the digital age. Reference \& User Services Quarterly, 39, 376-379.

Summey, T., \& Fisk, J. (2003). Who's out there in cyberspace: Profiling the remote learner for service design. In P. B. Mahoney (Ed.), The Tenth Off-Campus Library Services Conference proceedings, Cincinnati, OH (pp. 389-395). Mount Pleasant, MI: Central Michigan University.

Swope, M. J., \& Katzer, J. (1972). The silent majority: Why they don't ask questions. $R Q, 12,161-166$.

Tabs, E. D. (2003). Distance education at degree-granting postsecondary institutions: 2000-2001. Washington: U.S. Department of Education, Institute of Education Sciences, National Center for Education Statistics. Retrieved July 21, 2003, from http://nces.ed.gov/pubsearch/pubsinfo.asp?pubid=2003017

Taylor, S. S. (2002, May 13). Is anything wrong with this picture? Chronicle of Higher Education, 14(20), 10-12.

Tennant, R. (1999, June 15). Of human and humane assistance. Library Journal, 124(11). Retrieved January 18, 2004, from http://www.libraryjournal.com/index.asp

Tennant, R. (2003, January 1). Revisiting digital reference. Library Journal, 128(1), 3839.

Tenopir, C. (2004a, December 1). Chat's positive side. Library Journal, 129(20), 42. Retrieved October 8, 2005, from Academic Search Premier database.

Tenopir, C. (1998, November). Impact of digital reference on librarians and library users. Online, 22(6), 84.

Tenopir, C. (2004b, November 1). Rethinking virtual reference. Library Journal, 129(18), 34. Retrieved October 9, 2005, from Academic Search Premier database.

Tenopir, C. (2001, July 1). Virtual reference services in a real world. Library Journal, $126(12), 38-40$.

Tesdell, K. (2000). Evaluating public library service - the mystery shopper approach. Public Libraries, 39, 145.

Thomas, J. (2000). Mystery shoppers at the library. Retrieved June 24, 2004, from http://www.csulb.edu/divisions/aa/grad_undergrad/senate/assessment/grants/ thomas_99.pdf 
Thomsett-Scott, B. (2004). If you ask, I will tell you. In R. D. Lankes, J. Janes, L. C. Smith, \& C. M. Finneran (Eds.), The virtual reference experience: Integrating theory into practice (pp. 63-86). New York: Neal-Schuman.

Tibbo, H. R. (1995). Interviewing techniques for remote reference: Electronic versus traditional environments. American Archivist, 58, 294-310.

Tipton, C. J. (2001). Graduate students' perceptions of library support services for distance learners: A university system-wide study. Journal of Library Administration, 32, 393-408.

Tomer, C. (1994). MIME and electronic reference services. Reference Librarian, (41/42), 347-373.

Toraki, K. (2002). Remote users in the virtual library: A need for diversification? In P. Brophy, S. Fisher, \& Z. Clarke (Eds.), Libraries without walls 4: The delivery of library services to distant users (pp. 208-216). London: Facet Publishing.

Tu, C., \& McIsaac, M. (2002). The relationship of social presence and interaction in online classes. American Journal of Distance Education, 16, 131-150.

Twidale, M. B., \& Nichols, D. M. (1998). Designing interfaces to support collaboration in information retrieval. Interacting with Computers, 10, 177-93.

Tyckoson, D. (2003). On the desirableness of personal relations between librarians and readers: The past and future of reference service. Reference Services Review, 31, $12-16$.

Tyckoson, D. A. (2001). What is the best model of reference service? Library Trends, 50, 183-196.

Tyckoson, D. A. (1999). What's right with reference. American Libraries, 30(5), 58.

Tygett, M., Lawson, V. L., \& Weessies, K. (1996). Using undergraduate marketing students in an unobtrusive reference evaluation. $R Q, 36,270-276$.

U.S. Department of Education, National Center for Education Statistics. (2004). The condition of education. Washington: U.S. Government Printing Office.

U.S. Department of Education, Office of Educational Research and Improvement, National Center for Education Statistics. (2002). The condition of education, 2002.

Retrieved November 2, 2003, from http://www.nces.ed.gov/pubs2002/2002025_5.pdf

U.S. Library of Congress. (2002, June). IFLA: Reference work section. Retrieved October 31, 2003, from http://www.loc.gov/rr/digiref/digirefguidelines.html 
University at Albany Libraries. (2002). Reference service policy. Retrieved January 6, 2004, from http://library.albany.edu/dewey/referencepolicy.html

University of Minnesota Libraries. (1998, August 28). What faculty say about distance learning and library support. Retrieved August 9, 2003, from http://www.lib.umn.edu/dist/testing/dlfocus.phtml

University of Oregon, Office of the Registrar. (n.d.). Random 3-digit code number generator. Retrieved March 15, 2004, from http://registrar.uoregon.edu/random.php

Upcraft, L., Terenzini, P., \& Kruger, K. (2000). Looking beyond the horizon: Trends shaping student affairs. Retrieved February 6, 2004, from http://www.acpa.nche.edu/ seniorscholars/trends/trends5.htm

Valentine, B. (1999, April). Students versus the research paper: What can we learn? In H. Thompson (Ed.), Proceedings of the ACRL Ninth National Conference, Detroit, MI. Chicago: Association of College \& Research Libraries. Retrieved December 2, 2002, from http://www.ala.org/acrl/valentine.pdf

Venkatalakshmi, S. K., \& Sonker, S.K. (2002, April). Creating an Internet resource catalogue: Quality resources in the area of business and management. In Proceedings MANLIBNET 4th National Convention on Paradigm of IT Application to Business and Management Libraries 4, Faridabad, India (pp. 82-89). Retrieved July 2, 2003, from http://eprints.rclis.org/archive/00000307/01/CAFDIS.pdf

Virtual Reference Desk. (2002). About VRD. Retrieved January 12, 2004, from http://www.vfrd.org/about.shtml

Vishik, C. (1999). Intermediation and quality uncertainty in the Internet environment. Journal of Education for Library and Information Science, 40, 263-281.

Visser, L., \& Visser, Y. L. (2000). Perceived and actual student support needs in distance education. Quarterly Review of Distance Education, 1(2), 109-117.

Ward, D. (2004). Measuring the completeness of reference transactions in online chats. Reference \& User Services Quarterly, 44(1), 46-56.

Ward, D., \& Kern, K. (2004, November). Training and management of virtual reference services: How RUSA standards can help. In Proceedings of the Virtual Reference Desk Conference 2004, Cincinnati, OH. Retrieved October 8, 2005, from http://www.vrd.org/proceedings/presentation.cfm?PID=322

Ware, S. (2004, June). New definition of reference: Survey results. Paper presented at the American Library Association Annual Conference, Orlando, FL. 
Wasik, J., \& Silverstein, J. (2001, November). Answer-mania: Is everyone an expert? A digital reference quality comparison. In Proceedings of the Virtual Reference Desk Conference, Chicago, IL. Retrieved January 17, 2004, from http://www.vrd.org/ conferences/VRD2001/proceedings/wasik.shtml

Wasik, J. M. (1999). Building and maintaining digital reference services. ERIC Digest. Syracuse, NY: ERIC Clearinghouse on Information and Technology. Retrieved October 7, 2003, from http://ericit.org/digests/EDO-IR-1999-04.shtml

Wasik, J. M. (2003). Digital reference evaluation. Retrieved October 7, 2003, from http://www.vrd.org/AskA/digref_assess.shtml

Watson, E. F. (n.d.). Library services to distance learners - The new professional paradigms. Retrieved August 15, 2005, from http://www.col.org/forum/PCFpapers/ watson.pdf

Web traffic. (2004, November/December). World Watch, 17(6). Retrieved March 23, 2005, from Academic Search Premier database.

Webster's universal college dictionary. (2001). New York: Random House Press.

Weech, T. L. (1984). Who's giving all those wrong answers? Reference Librarian, 11, 112-115.

Weech, T. L., \& Goldhor, H. (1982). Obtrusive versus unobtrusive evaluation of reference service in five Illinois public libraries. Library Quarterly, 52, 305-324.

Weissman, S. K. (2002). E-characteristics. Retrieved January 18, 2004, from http://www.gti.net/weissman/character.html

West, J. (2001, April). Customer relationship management and you. IIE Solutions, 33(4), 34-37.

Western Cooperative for Educational Telecommunications. (2003a, May 15). About the project. Retrieved September 27, 2003, from http://www.wcet.info/projects/laap/ about/about.asp

Western Cooperative for Educational Telecommunications. (2003b, May 20). Library services. Retrieved September 27, 2003, from http://www.wcet.info/projects/laap/ resources/library.asp

Western Cooperative for Educational Telecommunications. (1996). Principles of good practice for electronically offered academic degree and certificate programs. Denver, CO: Author. Retrieved September 23, 2003, from http://www.wcet.info/ projects/balancing/principles.asp 
Wheeler, J., \& Fournier, L. (2001). Working in the asynchronous environment: Two case studies. Journal of Library Administration, 32, 425-438.

Whisner, M. (2003). Practicing reference... learning from library science. Law Library Journal, 95, 295-301.

Whitaker, B. (1989). Electronic mail in the library: A perspective. Library Trends, 37, 357-365.

White, H. S. (1999a). Librarians and information technology: Which is the tail and which is the dog? Library Trends, 48, 264-277.

White, M. D. (1999b). Analyzing electronic question/answer services: Framework and evaluations of selected services. College Park, MD: University of Maryland.

White, M. D. (2001). Diffusion of an innovation: Digital reference service in Carnegie Foundation master's (comprehensive) academic institution libraries. Journal of Academic Librarianship, 27(3), 173-187.

White, M. D. (1981). Dimensions of the reference interview. $R Q, 20,373-381$.

Whitlatch, J. (2003). Policies for digital reference in the digital reference research agenda. In R. D. Lankes, A. Goodrum, \& S. Nicholson (Eds.), Publications in librarianship (pp. 84-102). Chicago: Association of College \& Research Libraries.

Whitlatch, J. B. (2000). Evaluating reference services: A practical guide. Chicago: American Library Association.

Whitlatch, J. B. (2001). Evaluating reference services in the electronic age. Library Trends, 50, 207-217.

Whitlatch, J. B. (2004). Reference policies as practiced: Implications for quality service. In Proceedings of the Virtual Reference Desk Conference 2004, Cincinnati, OH. Retrieved October 8, 2005, from http://www.vrd2004.org/proceedings/ presentation.cfm? PID=390

Whitlatch, J. B. (1989). Unobtrusive studies and the quality of academic library reference services. College \& Research Libraries, 50, 181-194.

Whyte, S. B. (1995). Spanning the distance: Using computer conferencing as part of a team-taught research/writing class. Reference Librarian, (51/52), 267-279.

Wiese, F. O., \& Borgendale, M. (1986). EARS: Electronic access to reference service. Bulletin of the Medical Library Association, 74, 300-304. 
Williams, C. (2002). Learning on-line: A review of recent literature in a rapidly expanding field. Journal of Further and Higher Education, 26, 263-272.

Wills, D. (2004). E-mail revealed: What research tell us. Library Administration \& Management, 18, 87-92.

Woodard, B. S. (1989). The effectiveness of an information desk staffed by graduate students and nonprofessionals. College \& Research Libraries, 50, 455-467.

Wyer, J. I. (1930). Reference work. Chicago: American Library Association.

Zafeiriou, G., Nunes, J. M., \& Ford, N. (2001). Using students' perceptions of participation in collaborative learning activities in the design of online learning environments. Education for Information, 19, 83-106.

Zanin-Yost, A. (2004). Digital reference: What the past how taught us and what the future will hold. Library Philosophy and Practice, 7(1). Retrieved August 15, 2005, from http://libr.unl.edu:2000/LPP/zanin-yost.htm 